Chasapis, M., Samaras, D. A., Theodoropoulos, K. \& Eleftheriadou, E.: The vascular flora of Mt Tzena (northern Greece). - Fl. Medit. 30: 55-63. 2020. - ISSN: 1120-4052 printed, 2240-4538 online.

Electronic Supplementary File 1. https://doi.org/10.7320/FlMedit30.055.1 Version of Record published online on 25 May 2020

\title{
The vascular flora of Mt Tzena
}

Floristic catalogue - The following abbreviations and symbols are used in the floristic catalogue:

$*$ : new record

?: presence in study area questionable (not included in floristic analysis)

boldface italics: presence in study area confirmed by the authors normal italics: literature report not confirmed

E: East of longitude (World Geodetic System 1984 in decimal degrees)

N: North of latitude (World Geodetic System 1984 in decimal degrees)

Chas.: leg. M. Chasapis (TAUF), followed by collection number

FH: Flora Hellenica

MFG: Mountain Flora of Greece

obs.: observed in the field (no collection available)

s.lat.: sensu lato

\section{PTERIDOPHYTA}

\section{ASPLENIACEAE}

Asplenium adiantum-nigrum L. subsp. adiantum-nigrum - $\mathrm{H}$ ros, $\mathrm{Pt}-\mathrm{Chas} .1728$ (28/9/2011, 22.18948 E, 41.11359 N, 740 m), Voliotis 1983: 161 - Rare. In thermophilous deciduous shrublands and forests.

*Asplenium adiantum-nigrum subsp. onopteris (L.) Heufl. - H ros, ME - Chas. 482 (25/4/2010, 22.19922 E, 41.11005 N, 820 m), Chas. 977 (13/3/2011, 22.20151 E, 41.11359 N, 930 m), Chas. 1721 (28/9/2011, 22.19001 E, 41.11585 N, 880 m) Sporadic. Shady and stony places of oak forests and thermophilous deciduous stands.

Asplenium ceterach L. - H ros, EA - Chas. 417 (17/10/2009, 22.23613 E, 41.13268 N, $1340 \mathrm{~m}$, open thermophilous deciduous stand), Voliotis 1983: 161 - Common. Stony places in forests and shrublands. Up to $1700 \mathrm{~m}$.

*Asplenium ruta-muraria L. - H ros, Ct - Chas. 398 (9/10/2009, 22.22749 E, 41.12192 $\mathrm{N}, 1200 \mathrm{~m}$, stony open ground) - Sporadic. On calcareous rocks up to $1900 \mathrm{~m}$.

*Asplenium septentrionale (L.) Hoffm. - H ros, Bo - Chas. 1719 (28/9/2011, 22.20074 E, $41.11133 \mathrm{~N}, 870 \mathrm{~m}$, phrygana at stony ground) - Rare. At stony open places and rock crevices up to $1900 \mathrm{~m}$.

*Asplenium trichomanes subsp. quadrivalens D. E. Mey. - H ros, EA - Chas. 166 (13/8/2009, 22.18554 E, 41.12244 N, 960 m, beech stand), Chas. 941 (8/8/2010, 
22.22242 E, 41.12428 N, 1090 m, thermophilous deciduous stand) - Common. At shady stony places of forests and shrublands, mainly on calcareous substrates.

Asplenium trichomanes L. subsp. trichomanes - H ros, Co - Chas. 990 (28/3/2011, 22.19086 E, 41.11541 N, 830 m, thermophilous deciduous stand on schist), Voliotis 1983: 161 - Rare.

Asplenium viride Huds. - H ros, Bo - Chas. 1326 (2/6/2011, 22.23737 E, 41.14865 N, $1650 \mathrm{~m}$, beech stand), Chas. 2192 (15/7/2012, $22.24253 \mathrm{E}, 41.15638 \mathrm{~N}, 1640 \mathrm{~m}$, beech stand), MFG1: 19 - Rare. At open, stony and rocky calcareous places above treeline, up to $2000 \mathrm{~m}$. Also in beech stands at treeline.

\section{ATHYRIACEAE}

*Athyrium filix-femina (L.) Roth - H ros, Co - Chas. 222 (24/8/2009, 22.21625 E, $41.12769 \mathrm{~N}, 1160 \mathrm{~m}$, beech forest), Chas. 258 (28/8/2009, $22.21838 \mathrm{E}, 41.16349 \mathrm{~N}$, $1920 \mathrm{~m}$, streambank above treeline) - Common in mesic beech forests. Sporadic above treeline on streambanks.

\section{CYSTOPTERIDACEAE}

Cystopteris fragilis (L.) Bernh. - H caesp, Co - Chas. 959 (19/9/2010, 22.18762 E, $41.15103 \mathrm{~N}, 1590 \mathrm{~m}$, beech forest), Chas. 1047 (11/4/2011, 22.20837 E, 41.11965 N, 1030 m, mixed beech - oak forest), Chas. 1331 (2/6/2011, 22.22865 E, 41.14959 N, 1690 m, stony streambank above the treeline), Voliotis 1983: 161 - Common in beech forests. Sporadic in oak forests and in scrub above treeline.

*Gymnocarpium dryopteris (L.) Newman - G rhiz, Ct - Chas. 260 (28/8/2009, $22.21988 \mathrm{E}, 41.16081 \mathrm{~N}, 1920 \mathrm{~m}$, rock in a shrubland above treeline), Chas. 1644 (23/8/2011, 22.21591 E, 41.16678 N, 2050 m, rocky place above treeline) - Rare. On metamorphic rocks (schists) above treeline.

\section{DENNSTAEDTIACEAE}

Pteridium aquilinum (L.) Kuhn - Grhiz, Cosmop. - Chas. 284 (10/9/2009, 22.26245 E, $41.12858 \mathrm{~N}, 790 \mathrm{~m}$, Pteridium thicket in the vegetation zone of oaks), Voliotis 1983: 161 - Common. Forms dense stands (Pteridium thickets) up to treeline.

\section{DRYOPTERIDACEAE}

Dryopteris dilatata (Hoffm.) A. Gray - G rhiz, ES - Biel \& Tan 2008: 292.

*Dryopteris filix-mas (L.) Schott - G rhiz, Co - Chas. 124 (6/8/2009, 22.18389 E, $41.15205 \mathrm{~N}, 1600 \mathrm{~m}$, roadside in beech forest), Chas. 259 (28/8/2009, $22.21988 \mathrm{E}$, $41.16081 \mathrm{~N}, 1920 \mathrm{~m}$, streambank above treeline) - Common in mesic beech stands. Sporadic at streambanks above treeline up to $2000 \mathrm{~m}$.

*Polystichum aculeatum (L.) Roth - G rhiz, EA - Chas. 993 (28/3/2011, 22.19043 E, $41.11369 \mathrm{~N}, 760 \mathrm{~m}$, streambank in oak - thermophilous deciduous stand) Common in mesic beech stands. Sporadic at streambanks above treeline up to 2000 $\mathrm{m}$.

*Polystichum lonchitis (L.) Roth - G rhiz, AA - Chas. 261 (28/8/0209, 22.21988 E, $41.16081 \mathrm{~N}, 1920 \mathrm{~m}$, streambank above treeline), Chas. 434 (22/11/2009, 22.19425 E, 41.13924 N, $1700 \mathrm{~m}$, beech forest) - Common in mesic beech stands. Sporadic at streambanks above treeline up to $2000 \mathrm{~m}$.

\section{EQUISETACEAE}


Equisetum arvense L. - G rhiz, Ct - Chas. 45 (11/7/2009, 22.18 E, 41.12893 N, 1000 m, swampy place in beech stand), Chas. 423 (19/10/2009, 22.2695 E, $41.15508 \mathrm{~N}$, $1230 \mathrm{~m}$, swampy place at roadside in beech stand), Voliotis 1983: 161 - Common. Swampy places and fens up to $1900 \mathrm{~m}$.

*Equisetum hyemale L. - G rhiz, Ct - Chas. 1012 (3/4/2011, 22.22044 E, 41.13578 N, $1380 \mathrm{~m}$, spring in beech stand), Chas. 1776 (19/4/2012, $22.22254 \mathrm{E}, 41.13883 \mathrm{~N}$, $1470 \mathrm{~m}$, streambank in beech stand) - Sporadic. Damp places in beech forests.

*Equisetum palustre L. - G rhiz, Ct - Chas. 1316 (2/6/2011, 22.21895 E, 41.14233 N, $1610 \mathrm{~m}$, swampy place above treeline), Chas. 1970 (4/6/2012, 22.20426 E, $41.11733 \mathrm{~N}, 970 \mathrm{~m}$, open swampy place in oak - thermophilous deciduous stand) Sporadic. Swampy places and fens up to $2000 \mathrm{~m}$.

*Equisetum telmateia Ehrh. - G rhiz, Ct - Chas. 305 (10/9/2009, 22.25447 E, 41.13756 $\mathrm{N}, 1220 \mathrm{~m}$, swampy place in beech stand), Chas. 1722 (28/9/2011, 22.19177 E, 41.11272 N, 710 m, swampy place in Alnus glutinosa stand) - Sporadic. In swampy places up to $1400 \mathrm{~m}$.

\section{OPHIOGLOSSACEAE}

*Botrychium lunaria (L.) Sw. - G rhiz, Ct - Chas. 81 (20/7/2009, 22.22348 E, $41.14663 \mathrm{~N}, 1800 \mathrm{~m})$, Chas. 1544 (12/7/2011, $22.2308 \mathrm{E}, 41.15359 \mathrm{~N}, 1860 \mathrm{~m})-$ Common. In subalpine grasslands.

*Ophioglossum vulgatum L. - G rhiz, Ct - Chas. 1875 (19/5/2012, 22.20718 E, 41.1108 N, $800 \mathrm{~m}$, Pteridium thicket in thermophilous deciduous stand) - Rare. In Pteridium thickets of lowland areas.

\section{POLYPODIACEAE}

Polypodium vulgare L. - G rhiz, Ct - Chas. 44 (11/7/2009, 22.18 E, 41.12893 N, 1000 m, beech stand), Chas. 219 (24/8/2009, 22.21625 E, 41.12769 N, 1160 m, beech stand), Voliotis 1983: 161 - Common at rocky places in beech forests. Sporadic at rocky streambanks above treeline.

\section{PTERIDACEAE}

*Allosorus persicus (Bory) Christenh. - G rhiz, Me - Chas. 430 (14/11/2009, 22.22167 E, $41.1167 \mathrm{~N}, 840 \mathrm{~m}$, rocky streambank in thermophilous deciduous stand, Chas. $1075(22 / 4 / 2011,22.23657$ E, $41.12522 \mathrm{~N}, 1110 \mathrm{~m})$ - Common. At stony places and rocks in lowland phrygana.

*Paragymnopteris marantae (L.) K. H. Shing - H ros, ST - Chas. 367 (20/9/2009, 22.1941 E, 41.10573 N, 660 m, roadside in Juniperus oxycedrus - thermophilous deciduous shrubland), Chas. 1003 (31/3/2011, 22.27802 E, 41.148 N, 980 m, opening with phrygana in oak stand) - Sporadic. In lowland phrygana.

\section{GYMNOSPERMAE}

\section{CUPRESSACEAE}

Juniperus communis subsp. nana (Willd.) Syme $-\mathrm{P}$ caesp/ P scap, $\mathrm{Ct}-\mathrm{Chas} .75$ (20/7/2009, 22.21401 E, 41.14432 N, 1760 m), Chas. 1586 (22/7/2011, 22.17792 E, 41.15101 N, $1700 \mathrm{~m})$, Chas. 2311 (18/6/2013, 22.23321 E, $41.1394 \mathrm{~N}, 1760 \mathrm{~m})$, MFG1: 46 (as J. communis subsp. alpina (Suter) Celak.), FH1: map 18, Schuler 
2004: tab. 21 - Common. Covers extended areas above treeline. Forms low shrublands above $1400 \mathrm{~m}$.

*Juniperus communis subsp. hemisphaerica (C. Presl) Nyman - P caesp/ P scap, Me Chas. $421(17 / 10 / 2009,22.24312 \mathrm{E}, 41.13865 \mathrm{~N}, 1400 \mathrm{~m}$, shrubland at the edge of beech stand), Chas. 1066 (16/4/2011, 22.24759 E, 41.14088 N, 1470 m, shrubland at the edge of beech stand) - Rare.

*Juniperus foetidissima Willd. - P scap/P caesp, BA - Chas. 403 (9/10/2009, 22.22813 E, 41.11995 N, 1110 m, open thermophilous deciduous stand) - Rare.

Juniperus oxycedrus subsp. deltoides (R. P. Adams) N. G. Passal. - P scap/ P caesp, EM - Chas. 1703 (18/9/2011, 22.21597 E, 41.11166 N, 730 m, phrygana - juniper formation), MFG1: 47 (as $J$. oxycedrus L. subsp. oxycedrus), FH1: map 19 (as $J$. oxycedrus L. subsp. oxycedrus) - Common. In mixture with thermophilous deciduous and oak species or forming stands in forest openings. Sporadic in beech stands, in openings of beech forests and above treeline up to $1750 \mathrm{~m}$.

Juniperus sabina L. - P caesp/P scap, EA - Chas. 415 (17/10/2009, 22.24312 E, $41.13865 \mathrm{~N}, 1400 \mathrm{~m}$, shrubland at the edge of beech stand), MFG1: 48, FH1: map 25 - Sporadic. In shrublands within beech forests and above treeline. On calcareous substrates between 1400 and $1900 \mathrm{~m}$.

\section{EPHEDRACEAE}

*Ephedra foeminea Forssk. - P lian, Me - Chas. 972 (31/10/2010, 22.23384 E, $41.12058 \mathrm{~N}, 980 \mathrm{~m}$, stony place with phrygana) - Rare.

*Ephedra nebrodensis Guss subsp. nebrodensis - P caesp, Me - Chas. 413 $(17 / 10 / 2009,22.24211 \mathrm{E}, 41.13188 \mathrm{~N}, 1270 \mathrm{~m}$, stony place with open phrygana Juniperus oxycedrus), Chas. 984 (23/3/2011, 22.22657 E, 41.1293 N, 1600 m, open stony place) - Sporadic. On stony calcareous places with sparse vegetation. Between 1200 and $1700 \mathrm{~m}$.

\section{PINACEAE}

*Abies borisii-regis Mattf. - P scap, Bk - Chas. 422 (19/10/2009, 22.27019 E, 41.16031 N, $1450 \mathrm{~m}$, beech stand), Chas. 781 (12/6/2010, $22.18511 \mathrm{E}, 41.15963 \mathrm{~N}, 1820 \mathrm{~m}$, subalpine shrubland) - Sporadic. In beech stands and subalpine shrublands. Occasionally forms pure stands. Between 1000 and $2000 \mathrm{~m}$.

*Pinus nigra Arnold subsp. nigra - P scap, Me - Chas. 286 (10/9/2009,22.25174 E, $41.14175 \mathrm{~N}, 1420 \mathrm{~m}$, mixed stand of the species with Pinus sylvestris and Fagus sylvatica) - Sporadic. In forests, forest openings and subalpine shrublands. Between 700 and $2050 \mathrm{~m}$.

Pinus sylvestris L. - P scap, EA- Chas. 285 (10/9/2009, 22.25174 E, 41.14175 N, 1420 $\mathrm{m}$, mixed stand of the species with Pinus nigra and Fagus sylvatica), MFG1: 43 Sporadic. In forests, forest openings and subalpine shrublands. $800-2100 \mathrm{~m}$.

\section{TAXACEAE}

Taxus baccata L. - P scap, EA- Voliotis 1986: 50.

\section{(ANGIOSPERMAE) DICOTYLIDONEAE}

\section{$\underline{A C E R A C E A E}$}


*Acer campestre L. - P scap, EA - Chas. 1709 (24/9/2011, 22.24221 E, 41.11936 N, $790 \mathrm{~m}$, stream in thermophilous deciduous stand) - Rare.

*Acer heldreichii Orph. - P scap, Bk- Chas. 762 (12/6/2010, 22.17317 E, 41.15206 N, $1840 \mathrm{~m}$, open rocky place above treeline) - Rare.

*Acer hyrcanum Fischer \& C. A. Meyer - P scap, Bk - Chas. 35 (11/7/2009, 22.19068 E, $41.11748 \mathrm{~N}, 970 \mathrm{~m}$, thermophilous deciduous stand), Chas. 297 (10/9/2009, 22.26008 E, 41.12827 N, $810 \mathrm{~m}$, Quercus frainetto stand), Chas. 886 (24/7/2010, 22.24752 E, $41.14808 \mathrm{~N}, 1560 \mathrm{~m}$, beech - maple stand), Chas. 1515 (2/7/2011, 22.27605 E, 41.14757 N, 1060 m, oak stand) - Common. In deciduous forests and shrublands. Mainly on calcareous ground.

*Acer monspessulanum L. - P scap, ME - Chas. 623 (19/5/2010, 22.2155 E, 41.11597 N, $980 \mathrm{~m}$, thermophilous deciduous stand) - Sporadic. In oak and thermophilous deciduous stands.

*Acer platanoides L. - P scap, EA - Chas. 817 (26/6/2010, 22.2247 E, 41.11485 N, 760 m, thermophilous deciduous stand), Chas. 1986 (4/6/2012, 22.18926 E, 41.11775 $\mathrm{N}, 970 \mathrm{~m}$, thermophilous deciduous stand) - Sporadic. In forests all-over the mountain.

*Acer pseudoplatanus L. - P scap, Eu - Chas. 147 (6/8/2009, 22.19132 E, 41.14649 N, $1560 \mathrm{~m})$ - Sporadic. In beech stands.

\section{ADOXACEAE}

Adoxa moschatellina L. - G rhiz, ES - Chas. 1151 (6/5/2011, 22.25496 E, 41.15036 N, $1650 \mathrm{~m}$, beech stand at treeline), Chas. 1777 (19/4/2012, $22.20269 \mathrm{E}, 41.13145 \mathrm{~N}$, $1550 \mathrm{~m}$, beech stand), Chas. 1876 (19/5/2012, $22.23103 \mathrm{E}, 41.15855 \mathrm{~N}, 1690 \mathrm{~m}$, streambank with grassy vegetation above treeline), Parent 2005: 232 - Sporadic in mesic beech stands. Rare in shrublands and tall grasslands above treeline. 1400 $1900 \mathrm{~m}$.

\section{AMARANTHACEAE}

*Amaranthus hybridus L. - T scap, [N-Am.] - Chas. 966 (26/9/2010, 22.27482 E, $41.13822 \mathrm{~N}, 890 \mathrm{~m}$, roadside in oak forest) - Rare.

\section{ANACARDIACEAE}

*Pistacia terebinthus L. - P caesp/scap, Me - Chas. 394 (9/10/2009, 22.22604 E, $41.11388 \mathrm{~N}, 780 \mathrm{~m}$, thermophilous deciduous - Juniperus oxycedrus shrubland) Rare. In lowland thermophilous deciduous shrublands, on stony ground.

\section{APIACEAE}

*Aegopodium podagraria L. - G rhiz, ES - Chas. 53 (11/7/2009, 22.18 E, $41.12893 \mathrm{~N}$, 1000 m), Chas. 88 (27/7/2009, 22.1844 E, 41.1335 N, 1190 m) - Common. In beech stands on mesic to damp ground, also in Pteridium thickets at treeline and lower areas.

*Angelica sylvestris L. - H scap, EA - Chas. 1563 (22/7/2011, 22.17448 E, 41.15613 $\mathrm{N}, 1900 \mathrm{~m}$, streambank above treeline), Chas. 1616 (18/8/2011, 22.21262 E, 41.13439 N, $1460 \mathrm{~m}$, ditch by roadside in beech stand) - Rare. Streams and other damp places in beech stands and above treeline.

*Anthriscus cerefolium (L.) Hoffm. - T scap, EA - Chas. 1525 (7/7/2011, 22.19623 E, $41.1068 \mathrm{~N}, 720 \mathrm{~m}$, thermophilous deciduous stand), Chas. 1959 (4/6/2012, 
22.20377 E, 41.11786 N, 980 m, grassy, damp ground in thermophilous deciduous stand) - Sporadic. In forests and shrublands of lowland areas.

*Anthriscus sylvestris subsp. nemorosus (M. Bieb.) Koso-Pol. - Hscap, Pt - Chas. 753 (12/6/2010, 22.17892 E, 41.15089 N, 1690 m, grassland at treeline) - Sporadic. In grassy openings and roads of beech forests and in mesic grasslands at treeline.

*Bupleurum apiculatum Friv. - T scap, Bk - Chas. 1963 (4/6/2012, 22.25445 E, $41.10684 \mathrm{~N}, 600 \mathrm{~m})$ - Sporadic. In grasslands of lowland areas.

Bupleurum falcatum subsp. cernuum (Ten.) Arcang. - Hscap, ES - Chas. 896 $(24 / 7 / 2010,22.23944 \mathrm{E}, 41.14264 \mathrm{~N}, 1670 \mathrm{~m}$, grassland at treeline), Chas. 1587 (22/7/2011, 22.175 E, $41.15613 \mathrm{~N}, 1870 \mathrm{~m}$, shrubland at treeline), MFG1: 690 Common. In grasslands and shrublands at treeline and above.

*Bupleurum praealtum L. - T scap, Eu - Chas. 167 (13/8/2009, 22.18554 E, 41.12244 N, $950 \mathrm{~m}$, grassy opening in beech stand), Chas. 1201 (20/5/2011, 22.22748 E, 41.11346 N, 760 m, phrygana) - Sporadic. In grasslands and phrygana of lowland areas.

*Bupleurum trichopodum Boiss. \& Spruner - T scap, Me - Chas. 2437 (28/6/2014, 22.23667 E, $41.10801 \mathrm{~N}, 640 \mathrm{~m}$ ) - Rare. In dry grasslands of lowland areas.

Carum appuanum subsp. bulgaricum (Hartvig) Bechi \& Garbari - H scap, Bk - Chas. 936 (8/8/2010, 22.22778 E, 41.12886 N, 1520 m), Chas. 1542 (12/7/2011, 22.22774 E, 41.15377 N, 1830 m), Parent 2005: 211, MFG1: 698 (as C. rigidulum subsp. bulgaricum Hartvig), Schuler 2004: tab. 21 - Common in grasslands on stony ground. Sporadic in subalpine shrublands, between 1400 and $2150 \mathrm{~m}$.

*Chaerophyllum aureum L. - H scap, ME - Chas. 144 (6/8/2009, 22.19142 E, $41.14685 \mathrm{~N}, 1560 \mathrm{~m}$, grassy opening in beech stand at treeline), Chas. 1554 (17/7/2011, 22.19133 E, $41.1328 \mathrm{~N}, 1390 \mathrm{~m}$, streambank in beech stand) Common. In grasslands and Pteridium thickets in mesic ground at beech zone and in tall grasslands at treeline and above.

Chaerophyllum hirsutum L. - H scap, Eu - Chas. 1565 (22/7/2011, 22.17604 E, 41.15589 N, $1830 \mathrm{~m}$, streambank above treeline), Chas. 2125 (30/6/2012, 22.22101 E, $41.15884 \mathrm{~N}, 1860 \mathrm{~m}$, tall-herb vegetation in streambank above treeline), Chas. 2404 (25/7/2013, 22.21468 E, 41.16379 N, 1990 m, streambank above treeline), Voliotis 1983: 162 - Sporadic. In grasslands and Pteridium thickets within beech forests and in tall-herb vegetation at and above treeline. On mesic ground.

*Chaerophyllum nodosum (L.) Crantz - T scap, MS - Chas. 1419 (16/6/2011, 22.22418 E, 41.11629 N, 790 m, streambank in thermophilous deciduous stand) Rare. At streambanks, mainly with riparian tree vegetation, of lowland areas.

*Chaerophyllum temulum L. - T scap, EA - Chas. 1418 (16/6/2011, 22.22418 E, $41.11629 \mathrm{~N}, 790 \mathrm{~m}$, streambank in thermophilous deciduous stand), Chas. 1946 (30/5/2012, 22.19818 E, $41.10886 \mathrm{~N}, 790 \mathrm{~m}$, damp place in thermophilous deciduous stand) - Common in beech stands, mainly in open places. Sporadic in oak and thermophilous deciduous stands on mesic ground.

*Daucus carota subsp. major (Vis.) Arcang. - Hscap, ME - Chas. 902 (1/8/2010, 22.21645 E, $41.11166 \mathrm{~N}, 730 \mathrm{~m}$, forest road in thermophilous deciduous stand with Juniperus oxycedrus), Chas. 1611 (18/8/2011, 22.19793 E, 41.11813 N, 1070 m, forest road in mixed beech - thermophilous deciduous stand) - Sporadic. In forest roads up to $1200 \mathrm{~m}$ and in grasslands of lowland areas.

*Daucus guttatus Sm. subsp. guttatus - T scap, Me - Chas. 1444 (22/6/2011, 22.22068 E, $41.10921 \mathrm{~N}, 640 \mathrm{~m}$, grassland), Chas. 1494 (2/7/2011, 22.26744 E, $41.12442 \mathrm{~N}$, 
$680 \mathrm{~m}$, grassland), Chas. 1519 (7/7/2011, 22.22487 E, $41.11306 \mathrm{~N}, 710 \mathrm{~m}$, phrygana) - Sporadic. Grasslands and phrygana of lowland areas, sometimes abundant.

*Dichoropetalum oligophyllum (Griseb.) Pimenov \& Kljuykov - H scap, Bk - Chas. $80(20 / 7 / 2009,22.22028$ E, 41.15955 N, 1900 m), Chas. $263(28 / 8 / 2009,22.2207$ E, $41.16145 \mathrm{~N}, 1890 \mathrm{~m})$, Chas. $910(1 / 8 / 2010,22.21933 \mathrm{E}, 41.15927 \mathrm{~N}, 1950 \mathrm{~m})-$ Common. subalpine grasslands. Above $1750 \mathrm{~m}$.

*Eryngium amethystinum L. - H scap, Me - Chas. 178 (13/8/2009, 22.18691 E, $41.1203 \mathrm{~N}, 950 \mathrm{~m}$, grassy opening in beech stand), Chas. $352(15 / 9 / 2009,22.26424$ E, $41.16013 \mathrm{~N}, 1540 \mathrm{~m}$, grassland at treeline) - Common. In grasslands from 600 up to $1900 \mathrm{~m}$.

*Eryngium campestre L. - H scap, EA - Chas. 1550 (17/7/2011, 22.22138 E, 41.10967 N, 650 m, dry grassland), Chas. 1671 (31/8/2011, 22.21678 E, 41.11257 N, 750 m, forest road in thermophilous deciduous stand with Juniperus oxycedrus) Sporadic. In dry grasslands and forest roads of lowland areas.

*Ferulago sylvatica (Besser) Rchb. - H scap, BI - Chas. 2001 (8/6/2012, 22.21834 E, $41.11647 \mathrm{~N}, 950 \mathrm{~m}$, phrygana with Juniperus oxycedrus) - Sporadic. Thermophilous deciduous stands, oak forests and Juniperus oxycedrus shrublands on calcareous substrates.

*Geocaryum capillifolium (Guss.) Coss. - G bulb, BI - Chas. 2342 (26/6/2013, 22.20204 E, 41.13333 N, 1630 m, Pteridium thicket in beech stand) - Rare.

Hellenocarum strictum (Griseb.) Hand - H scap, BI - Chas. 1996 (8/6/2012, 22.2183 E, $41.11809 \mathrm{~N}, 1050 \mathrm{~m}$, phrygana), Chas. 2297 (18/6/2013, 22.24332 E, 41.13559 $\mathrm{N}, 1330 \mathrm{~m}$, sparse grassy clearing in thermophilous deciduous stand), MFG1: 696 (as Carum strictum (Griseb.) Boiss.) - Sporadic. In sparse grasslands and phrygana on stony calcareous ground. Between 1000 and $1750 \mathrm{~m}$.

*Heracleum sphondylium subsp. ternatum (Velen.) Brummitt. - H scap, Me - Chas. $141(6 / 8 / 2009,22.19305 \mathrm{E}, 41.1485 \mathrm{~N}, 1580 \mathrm{~m}$, forest road in beech stand) Common. In mesic to damp beech forests.

*Laserpitium siler subsp. laeve (Halácsy) Hartvig - H scap, Gr - Chas. 928 (8/8/2010, 22.22563 E, $41.1291 \mathrm{~N}, 1530 \mathrm{~m})$, Chas. 2353 (1/7/2013, 22.22454 E, $41.12971 \mathrm{~N}$, $1510 \mathrm{~m}$ ) - Sporadic. In open beech stands on stony or rocky calcareous ground. Up to $1650 \mathrm{~m}$.

*Malabaila involucrata Boiss. \& Spruner - H scap, Bk - Chas. 612 (19/5/2010, 22.2155 E, $41.11597 \mathrm{~N}, 980 \mathrm{~m}$, open thermophilous deciduous stand), Chas. 1862 $(16 / 5 / 2012,22.22181 \mathrm{E}, 41.11157 \mathrm{~N}, 690 \mathrm{~m}$, forest road in thermophilous deciduous stand with Juniperus oxycedrus) - Sporadic. In forest roads, open thermophilous deciduous stands, shrublands of Juniperus oxycedrus and phrygana of lowland areas.

*Orlaya daucoides (L.) Greuter - T scap, MS - Chas. 1200 (20/5/2011, 22.22559 E, $41.11262 \mathrm{~N}, 700 \mathrm{~m}$ ) - Common. In thermophilous deciduous stands, phrygana and shrublands with Juniperus oxycedrus, in lowland areas.

*Orlaya grandiflora (L.) Hoffm. - T scap, EA - Chas. 830 (26/6/2010, 22.2213 E, $41.11705 \mathrm{~N}, 840 \mathrm{~m}$, open thermophilous deciduous stand), Chas. 1404 (16/6/2011, 22.22675 E, $41.11381 \mathrm{~N}, 800 \mathrm{~m}$, phrygana), Chas. 1445 (22/6/2011, 22.22068 E, $41.10921 \mathrm{~N}, 640 \mathrm{~m}$, dry grassland), Chas. 1469 (27/6/2011, 22.2148 E, $41.11101 \mathrm{~N}$, $720 \mathrm{~m}$, forest road in thermophilous deciduous stand with Juniperus oxycedrus) Common along roadsides and grasslands of lowland areas. Sporadic in open 
thermophilous deciduous and Juniperus oxycedrus shrublands and in phrygana, on stony ground.

*Peucedanum aegopodioides Seidel - H scap, BA - Chas. 371 (20/9/2009, 22.18372 E, 41.12394 N, $950 \mathrm{~m}$, stream in beech stand), Chas. 1726 (28/9/2011, 22.19177 E, 41.11272 N, 720 m, alder stand) - Rare.

*Peucedanum austriacum (Jacq.) W. D. J. Koch - H scap, ME - Chas. 168 (13/8/2009, $22.18554 \mathrm{E}, 41.12244 \mathrm{~N}, 950 \mathrm{~m}$, grassy opening in beech stand), Chas. 933 (8/8/2010, 22.22778 E, 41.12886 N, 1520 m, grassland), Chas. 2184 (11/7/2012, $22.19621 \mathrm{E}, 41.11576 \mathrm{~N}, 1020 \mathrm{~m}$, open thermophilous deciduous stand), Chas. 2460 (20/7/2014, 22.22316 E, 41.13663 N, 1470 m, beech stand) - Sporadic. In grasslands and open deciduous broad-leaved stands, on stony ground. Between 600 and $1600 \mathrm{~m}$.

*Physospermum cornubiense (L.) DC. - H scap, EA - Chas. 1729 (28/9/2011, 22.19237 E, $41.11246 \mathrm{~N}, 720 \mathrm{~m}$, thermophilous deciduous stand), Chas. 1987 (4/6/2012, 22.20219 E, 41.11509 N, 970 m, Quercus frainetto stand), Chas. 2149 (6/7/2012, 22.28485 E, 41.14225 N, 780 m, Quercus frainetto stand) - Common in oak forests. Sporadic in thermophilous deciduous stands.

*Pimpinella saxifraga L. - H scap, ES - Chas. 923 (1/8/2010, 22.218 E, 41.16006 N, $1990 \mathrm{~m}$, subalpine grassland), Chas. 1589 (22/7/2011, 22.17672 E, $41.15249 \mathrm{~N}$, $1770 \mathrm{~m}$, subalpine shrubland) - Common. In subalpine grasslands and shrublands. Between 1700 and $2180 \mathrm{~m}$.

* Pimpinella tragium subsp. polyclada (Boiss. \& Heldr.) Tutin - C suffr, BA - Chas. $374(27 / 9 / 2009,22.21549 \mathrm{E}, 41.11183 \mathrm{~N}, 740 \mathrm{~m}$, forest road in thermophilous deciduous stand with Juniperus oxycedrus), Chas. 934 (8/8/2010, 22.22798 E, $41.1258 \mathrm{~N}, 1370 \mathrm{~m}$, grassy clearing in thermophilous deciduous stand) - Sporadic. In grasslands, phrygana and forest roads of lowland areas.

*Sanicula europaea L. - H scap, ES - Chas. 217 (24/8/2009, 22.21662 E, 41.12743 N, $1140 \mathrm{~m}$, beech stand), Chas. 1192 (20/5/2011, $22.22396 \mathrm{E}, 41.11592 \mathrm{~N}, 780 \mathrm{~m}$, stream in mixed beech - thermophilous deciduous stand) - Common. In mesic beech stands.

*Scandix australis L. subsp. australis - T scap, ME - Chas. 617 (19/5/2010, 22.21624 E, 41.11932 N, $1110 \mathrm{~m}$, sparse shrubland of Juniperus oxycedrus) - Sporadic. In sparse shrublands, phrygana and grasslands of lowland areas.

*Selinum silaifolium (Jacq.) Beck - H scap, ME - Chas. 818 (26/6/2010, 22.22359 E, $41.11637 \mathrm{~N}, 790 \mathrm{~m}$, rocky opening in thermophilous deciduous stand), Chas. 889 $(24 / 7 / 2010,22.22606 \mathrm{E}, 41.13523 \mathrm{~N}, 1630 \mathrm{~m}$, sparse grassland on rocky slope at treeline), Chas. $1472(27 / 6 / 2011,22.21415 \mathrm{E}, 41.12181 \mathrm{~N}, 1080 \mathrm{~m}$, rocky opening in thermophilous deciduous stand), Chas. 2461 (20/7/2014, 22.22316 E, 41.13663 $\mathrm{N}, 1470 \mathrm{~m}$, beech stand on stony ground) - Common in forest openings, forest stands and grasslands, mainly on stony calcareous ground. $900-1800 \mathrm{~m}$.

Seseli peucedanoides Koso-Pol. - H scap, ES - Chas. 935 (8/8/2010, 22.2278 E, $41.12886 \mathrm{~N}, 1520 \mathrm{~m}$, grassland), Chas. 1511 (2/7/2011, $22.27718 \mathrm{E}, 41.14826 \mathrm{~N}$, $1010 \mathrm{~m}$, open thermophilous deciduous stand), MFG1: 682 - Sporadic. On stony ground in different vegetation types, between 900 and $1600 \mathrm{~m}$.

*Tordylium apulum L. - Tscap, Me - Chas. 1597 (13/8/2011, 22.22671 E, 41.11101 N, $660 \mathrm{~m}$, grassland) - Sporadic. At roadsides and grasslands of lowland areas.

*Torilis arvensis subsp. neglecta (Spreng.) Thell. - T scap, ME - Chas. 2148 (6/7/2012, 22.28378 E, 41.14205 N, 780 m, roadside in Quercus frainetto stand) - Rare. 
*Torilis japonica (Houtt.) DC. - T scap, ES - Chas. $115(27 / 7 / 2009,22.1844$ E, $41.1335 \mathrm{~N}, 1190 \mathrm{~m}$, roadside in beech stand), Chas. $218(24 / 8 / 2009,22.21662 \mathrm{E}$, $41.12743 \mathrm{~N}, 1140 \mathrm{~m}$, roadside in beech stand) - Sporadic. At roadsides in beech, oak and thermophilous deciduous stands.

*Trinia glauca L. subsp. glauca - H scap, Eu - Chas. 618 (19/5/2010, 22.21455 E, $41.11587 \mathrm{~N}, 990 \mathrm{~m}$, sparse grassland on rocky ground), Chas. 1127 (1/5/2011, 22.22112 E, $41.11516 \mathrm{~N}, 850 \mathrm{~m}$, phrygana) - Common. In grasslands and phrygana on stony to rocky ground. Between 600 and $2100 \mathrm{~m}$.

\section{ARALIACEAE}

Hedera helix Lowe subsp. helix - P lian, ME - Chas. 411 (17/10/2009, 22.24621 E, $41.13455 \mathrm{~N}, 1180 \mathrm{~m}$, beech stand), Voliotis 1983: 162 - Common. In mesic beech forests and Platanus stands. Between 600 and $1500 \mathrm{~m}$.

\section{ARISTOLOCHIACEAE}

Aristolochia rotunda L. - G bulb, Me - Chas. 665 (24/5/2010, 22.26583 E, 41.15385 $\mathrm{N}, 1200 \mathrm{~m}$, damp place at roadside in beech stand), Chas. 1037 (8/4/2011, 22.1959 E, $41.1054 \mathrm{~N}, 670 \mathrm{~m}$, thermophilous deciduous stand), FH1: map 124 - Sporadic. In thermophilous deciduous stands.

\section{ASCLEPIADACEAE}

*Vincetoxicum fuscatum (Hornem.) Rchb. f. - H scap, EA - Chas. 841 (26/6/2010, 22.22222 E, 41.12734 N, $1340 \mathrm{~m}$, open shrubland of Juniperus oxycedrus and thermophilous deciduous on rocky slope) - Rare.

Vincetoxicum hirundinaria subsp. nivale (Boiss. \& Heldr.) Markgr. - H scap, Bk Chas. $1254(25 / 5 / 2011,22.21715$ E, $41.12086 \mathrm{~N}, 1120 \mathrm{~m}$, open thermophilous deciduous shrubland on rocky slope), Chas. 1993 (8/6/2012, 22.21742 E, 41.1197 N, 1150 m, sparse grassland on rocky slope), Schuler 2004: tab. 20 - Sporadic. At rocky or stony places with sparse vegetation of low and mid-altitudes.

*Vincetoxicum speciosum Boiss. \& Spruner - G rhiz, BA - Chas. 1504 (2/7/2011, 22.27967 E, 41.14875 N, 970 m, Quercus frainetto stand) - Sporadic. In dry oak and beech stands.

\section{ASTERACEAE}

Achillea ageratifolia subsp. aizoon (Griseb.) Heimerl - H scap, Bk - Chas. 670 $(24 / 5 / 2010,22.24558 \mathrm{E}, 41.15805 \mathrm{~N}, 1800 \mathrm{~m}$, sparse subalpine grassland on stony ground), MFG2: 435 - Sporadic. Stony places with sparse vegetation above treeline.

*Achillea coarctata Poir. - H scap, Eu - Chas. 322 (10/9/2009, 22.26111 E, 41.13433 N, $950 \mathrm{~m}$, forest road in oak stand), Chas. 688 (2/6/2010, $22.19349 \mathrm{E}, 41.11518 \mathrm{~N}$, $910 \mathrm{~m}$, forest road in thermophilous deciduous stand), Chas. 1524 (7/7/2011, 22.19623 E, $41.1068 \mathrm{~N}, 720 \mathrm{~m}$, thermophilous deciduous stand) - Common in forest roads of lowland areas. Sporadic in thermophilous deciduous and Juniperus oxycedrus shrublands and oak forests and in grasslands on stony ground, up to 1800 $\mathrm{m}$.

*Achillea crithmifolia Waldst. \& Kit. - H scap, BC - Chas. 689 (2/6/2010, 22.19336 E, $41.11536 \mathrm{~N}, 910 \mathrm{~m}$, forest road in thermophilous deciduous stand), Chas. 1937 
$(30 / 5 / 2012,22.19337 \mathrm{E}, 41.115 \mathrm{~N}, 910 \mathrm{~m}$, forest road in thermophilous deciduous stand) - Sporadic. In forest roads and dry grasslands of lowland areas.

*Achillea distans subsp. stricta (Gremli) Janch. - H scap, Eu - Chas. 65 (20/7/2009, 22.20928 E, $41.13867 \mathrm{~N}, 1680 \mathrm{~m}$, forest road in beech stand at treeline), Chas. 143 (6/8/2009, 22.19142 E, 41.14685 N, 1560 m, Juniperus communis subsp. nana shrubland at treeline), Chas. $330(15 / 9 / 2009,22.25928 \mathrm{E}, 41.16285 \mathrm{~N}, 1750 \mathrm{~m}$, subalpine grassland), Chas. 968 (26/9/2010, 22.2716 E, $41.13412 \mathrm{~N}, 840 \mathrm{~m}$, forest road in Quercus frainetto stand) - Common. At forest roads in oak and beech stands, in beech forests openings and grasslands above treeline. $800-2000 \mathrm{~m}$.

Achillea fraasii Sch. Bip. - H scap, BA - Chas. 616 (19/5/2010, 22.21658 E, 41.11554 N, 920 m, phrygana on stony ground), Chas. 847 (26/6/2010, 22.21802 E, 41.12412 $\mathrm{N}, 1110 \mathrm{~m}$, sparse grassland on rocky slope), MFG2: 439 - Common. Sparse grasslands and phrygana on stony to rocky calcareous ground. $900-1900 \mathrm{~m}$.

*Achillea grandifolia Friv. - H scap, BA - Chas. 92 (27/7/2009, 22.1844 E, 41.1335 N, $1190 \mathrm{~m}$ ) - Sporadic. In beech forests, mainly on forest roads and forest openings.

*Achillea holosericea Sm. - H scap, Bk - Chas. 332 (15/9/2009, 22.26009 E, 41.16376 $\mathrm{N}, 1800 \mathrm{~m}$, sparse subalpine grassland on stony ground) - Common in sparse grasslands at treeline and above, on rocky or stony calcareous ground. Sporadic in open thermophilous deciduous or beech stands, on rocky or stony ground. 800 $2050 \mathrm{~m}$.

*Achillea millefolium L. - H scap, ES - Chas. 307 (10/9/2009, 22.25447 E, 41.13756 $\mathrm{N}, 1210 \mathrm{~m}$, forest road in beech stand), Chas. $2040(18 / 6 / 2012,22.23391 \mathrm{E}$, $41.13094 \mathrm{~N}, 1320 \mathrm{~m}$, open thermophilous deciduous stand) - Sporadic. At forest roads and grassy openings in beech, oak and thermophilous deciduous forests and also in subalpine grasslands. Between 1100 and $2100 \mathrm{~m}$.

Antennaria dioica (L.) Gaertn. - C rept, ES - Chas. 703 (2/6/2010, 22.20122 E, $41.13737 \mathrm{~N}, 1790 \mathrm{~m})$, MFG2: 417 - Sporadic. On rocky and stony places with sparse vegetation above treeline and at treeline.

*Anthemis arvensis L. subsp. arvensis - T scap, EA/[Co] - Chas. 1982 (4/6/2012, 22.2531 E, 41.11268 N, 660 m, dry grassland) - Rare.

*Anthemis pindicola Halácsy - H scap, Bk - Chas. 745 (12/6/2010, 22.17429 E, $41.15442 \mathrm{~N}, 1890 \mathrm{~m}$, rocky opening in subalpine shrubland), Chas. 2219 $(31 / 7 / 2012,22.17683 \mathrm{E}, 41.15761 \mathrm{~N}, 1850 \mathrm{~m}$, rocky streambank above treeline) Sporadic. On stony places with sparse subalpine vegetation.

*Anthemis tinctoria L. subsp. tinctoria - H scap, Eu - Chas. 10 (11/7/2009, 22.18824 E, $41.11978 \mathrm{~N}, 1000 \mathrm{~m}$, forest road in beech stand), Chas. 201 (24/8/2009, $22.21376 \mathrm{E}, 41.12306 \mathrm{~N}, 1100 \mathrm{~m}$, forest road in beech stand), Chas. 424 $(19 / 10 / 2009,22.27125$ E, 41.16096 N, 1460 m, grassland at treeline) - Sporadic. In forest roads and grasslands up to $1500 \mathrm{~m}$.

*Arctium lappa L. - H bienn, ES - Chas. 243 (28/8/2009, 22.21245 E, 41.12746 N, $1290 \mathrm{~m}$, forest road in beech stand) - Sporadic. Roads in beech forests.

*Arctium minus (Hill) Bernh. - H bienn, ES - Chas. 16 (11/7/2009, 22.18 E, 41.12893 N, 1000 m), Chas. 189 (24/8/2009, 22.21376 E, 41.12306 N, 1090 m) - Sporadic. Roads in beech forests.

Artemisia vulgaris L. - H scap, Bk - Chas. 183 (13/8/2009, 22.18661 E, 41.12282 N, $990 \mathrm{~m}$, forest road in mixed beech - thermophilous deciduous stand), Schuler 2004: map 17 - Sporadic. At forest roads up to $1200 \mathrm{~m}$. 
Aster alpinus L. - H scap, Bk - Chas. 1631 (18/8/2011, 22.22826 E, 41.14644 N, 1880 m), Chas. 2079 (25/6/2012, 22.20799 E, 41.14684 N, $1970 \mathrm{~m})$, MFG2: 405, Schuler 2004: tab. 21 - Sporadic. Grasslands and open shrublands on stony ground above treeline.

Bellis perennis L. - H ros, EA - Chas. 500 (25/4/2010, 22.19832 E, 41.10823 N, 790 $\mathrm{m}$, grassy clearing in thermophilous deciduous stand), Voliotis 1983: 162 Sporadic. In grasslands and forest roads up to $1500 \mathrm{~m}$.

Bellis sylvestris Cirillo - H ros, Me - Voliotis 1983: 162.

*Carduus candicans Waldst. \& Kit. - H bienn, Bk - Chas. 2245 (1/6/2013, 22.26665 E, $41.12756 \mathrm{~N}, 720 \mathrm{~m}$, dry grassland) - Sporadic. In forest roads and dry grasslands of lowland areas.

*Carduus kerneri subsp. scardicus (Griseb.) Kazmi - H scap, Bk - Chas. 337 $(15 / 9 / 2009,22.25928 \mathrm{E}, 41.16285 \mathrm{~N}, 1750 \mathrm{~m}$, subalpine grassland), Chas. 885 (24/7/2010, 22.23944 E, 41.14264 N, 1670 m, open low shrubland of Juniperus communis subsp. nana at treeline), Chas. 922 (1/8/2010, 22.21896 E, $41.15953 \mathrm{~N}$, $1940 \mathrm{~m}$, subalpine grassland) - Common in grasslands at treeline and above. Sporadic at forest roads in oak and beech forests.

*Carduus nutans subsp. leiophyllus (Petrović) Stoj. \& Stef. - H scap, ES - Chas. 884 (24/7/2010, 22.23944 E, 41.14264 N, 1670 m, open low shrubland of Juniperus communis subsp. nana at treeline) - Sporadic. At forest roads, dry grasslands of lowland areas, grasslands at treeline and above.

Carduus personata subsp. albidus (Adamović) Kazmi - H scap, BC - Chas. 1645 $(23 / 8 / 2011,22.22101 \mathrm{E}, 41.15884 \mathrm{~N}, 1860 \mathrm{~m}$, tall-herb vegetation at margins of fen above treeline), Chas. 1694 (13/9/2011, 22.24694 E, 41.15177 N, 1770 m, tall-herb vegetation above treeline), Chasapis et al. 2019 - Rare. Tall grasslands on damp ground above treeline.

*Carduus pycnocephalus L. - H bienn/T scap, - Chas. 1675 (10/9/2011, 22.27334 E, $41.13631 \mathrm{~N}, 870 \mathrm{~m}$, forest road in Quercus frainetto stand), Chas. 2238 (9/8/2012, 22.20735 E, $41.13071 \mathrm{~N}, 1470 \mathrm{~m}$, forest road in beech stand) - Sporadic. At forest roads up to $1400 \mathrm{~m}$ and in dry grasslands of lowland areas.

Carlina biebersteinii subsp. brevibracteata (Andrae) $\mathrm{K}$. Werner - H scap, Eu - Chas. $128(6 / 8 / 2009,22.18389 \mathrm{E}, 41.15205 \mathrm{~N}, 1590 \mathrm{~m}$, forest road in beech stand), Voliotis 1983: 162 (as C. vulgaris L.) - Common. In grasslands and at forest roads at treeline and above, mainly on stony ground.

*Carlina corymbosa L. - H scap, Me - Chas. 1635 (23/8/2011, 22.21654 E, 41.11266 $\mathrm{N}, 760 \mathrm{~m}$, forest road in shrubland of Juniperus oxycedrus and thermophilous deciduous) - Common. At forest roads, dry grasslands and open shrublands of lowland areas.

*Carthamus lanatus L. - T scap, Me - Chas. 901 (1/8/2010, 22.21645 E, 41.11166 N, $730 \mathrm{~m}$, forest road in shrubland of Juniperus oxycedrus and thermophilous deciduous) - Rare.

Centaurea affinis Friv. subsp. affinis - H scap, Bk - Chas. 327 (15/9/2009, 22.25927 E, $41.16312 \mathrm{~N}, 1760 \mathrm{~m})$, Chas. 2135 (30/6/2012, $22.22554 \mathrm{E}, 41.15432 \mathrm{~N}, 1830 \mathrm{~m})$, MFG2: 496 - Sporadic. In sparse grasslands on stony ground above treeline and at treeline.

*Centaurea affinis subsp. pallidior (Halácsy) Hayek - H scap, Bk - Chas. 348 $(15 / 9 / 2009,22.26335 \mathrm{E}, 41.16228 \mathrm{~N}, 1670 \mathrm{~m}$, grassland on stony ground at treeline), Chas. 641 (19/5/2010, 22.21624 E, 41.11932 N, 1110 m, open shrubland 
of Juniperus oxycedrus and thermophilous deciduous), Chas. 1476 (27/6/2011, 22.21475 E, 41.12155 N, $1080 \mathrm{~m}$, open shrubland of Juniperus oxycedrus and thermophilous deciduous), Chas. 2009 (13/6/2012, 22.22678 E, 41.11705 N, 950 m, open shrubland of Juniperus oxycedrus and thermophilous deciduous) - Common at stony places of lowland areas with sparse shrubs or phrygana. Sporadic at similar places up to treeline.

*Centaurea cuneifolia subsp. pallida (Friv.) Hayek - H scap, Bk - Chas. 306 (10/9/2009, 22.24724 E, $41.13168 \mathrm{~N}, 1060 \mathrm{~m}$, forest road in oak stand), Chas. 1432 $(16 / 6 / 2011,22.22438 \mathrm{E}, 41.11332 \mathrm{~N}, 730 \mathrm{~m}$, phrygana on rocky ground), Chas. 1478 (27/6/2011, 22.21601 E, 41.11886 N, 1100 m, open shrubland of Juniperus oxycedrus and thermophilous deciduous) - Sporadic. In phrygana and open shrublands of lowland areas.

*Centaurea cyanus L. - T scap, Me - Chas. obs. (26/5/2014, 22.28721 E, 41.134 N, $690 \mathrm{~m}$, dry grassland) - Rare. In dry grasslands of lowland areas.

*Centaurea diffusa Lam. - T scap/H bienn, EA - Chas. 2200 (27/7/2012, 22.25705 E, 41.1076 N, 610 m, dry grassland) - Rare.

Centaurea grbavacensis (Rohlena) Stoj. \& Acht. - H scap, Bk - Chas. 416 (17/10/2009, 22.23613 E, 41.13268 N, $1340 \mathrm{~m}$, open thermophilous deciduous stand), Chas. 1914 (24/5/2012, 22.22509 E, 41.11351 N, 720 m, phrygana), MFG2: 493, Font et al. 2009: 987 - Sporadic. Stony and rocky calcareous places with sparse vegetation. Between 700 and $1800 \mathrm{~m}$.

*Centaurea grisebachii (Nyman) Heldr. subsp. grisebachii - H scap, Bk - Chas. 214 $(24 / 8 / 2009,22.21415 \mathrm{E}, 41.1219 \mathrm{~N}, 1090 \mathrm{~m}$, forest road in mixed beech thermophilous deciduous stand), Chas. 1477 (27/6/2011, 22.21647 E, $41.11959 \mathrm{~N}$, $1120 \mathrm{~m}$, open shrubland of thermophilous deciduous and Juniperus oxycedrus on rocky ground), Chas. 1549 (17/7/2011, 22.21737 E, 41.11285 N, 760 m, forest road in shrubland of thermophilous deciduous and Juniperus oxycedrus), Chas. 2182 $(11 / 7 / 2012,22.18652 \mathrm{E}, 41.13444 \mathrm{~N}, 1260 \mathrm{~m}$, forest road in beech stand) Sporadic. At forest roads, phrygana and open shrublands of lowland areas.

*Centaurea jacea subsp. angustifolia (DC.) Gremli - H scap, Eu - Chas. 1661 (31/8/2011, 22.1961 E, 41.11972 N, 1130 m, forest road in beech stand) - Rare.

*Centaurea kotschyana Heuff. - H scap, BC - Chas. 2226 (31/7/2012, 22.17162 E, 41.15195 N, 1920 m, grassland on a steep slope) - Rare.

*Centaurea napulifera Rochel subsp. napulifera - H scap, Bk - Chas. 678 (24/5/2010, 22.25003 E, 41.16109 N, $1860 \mathrm{~m}$, subalpine grassland), Chas. 2249 (1/6/2013, 22.27624 E, 41.14797 N, 1040 m, Quercus frainetto stand) - Sporadic. In oak and thermophilous deciduous forests and grasslands of mid-altitudes and above treeline.

?Centaurea napulifera subsp. nissana (Petrović) Dostál - H scap, Bk - Voliotis 1983: 162.

Centaurea napulifera subsp. tuberosa (Vis.) Stoj. \& Acht. - H scap, Bk - Chas. 585 $(12 / 5 / 2010,22.24872 \mathrm{E}, 41.13423 \mathrm{~N}, 1170 \mathrm{~m}$, mixed oak and beech stand), Chas. $632(19 / 5 / 2010,22.21656 \mathrm{E}, 41.11617 \mathrm{~N}, 970 \mathrm{~m}$, thermophilous deciduous stand with Juniperus oxycedrus), Chas. 2089 (25/6/2012, 22.22166 E, 41.14786 N, 1790 m, subalpine grassland), Chas. 2423 (26/5/2014, $22.27504 \mathrm{E}, 41.15282 \mathrm{~N}, 1100 \mathrm{~m}$, grassy opening in beech stand), Chasapis et al. 2019 - Common. In grasslands, in forest and shrubland margins and openings, between 700 and $2100 \mathrm{~m}$. 
*Centaurea nervosa Willd. subsp. nervosa - H scap, BC - Chas. 906 (1/8/2010, 22.2139 E, 41.16153 N, 2060 m), Chas. 2400 (25/7/2013, 22.20986 E, 41.16119 N, $2150 \mathrm{~m}$ ) - Rare. In open subalpine shrublands on siliceous substrates.

Centaurea phrygia subsp. indurata (Janka) Stoj. \& Acht. - H scap, BC - Strid \& Papanicolaou 1981: 79.

*Centaurea phrygia subsp. stenolepis (A. Kern.) Gugler - H scap, EA - Chas. 188 $(13 / 8 / 2009,22.18661 \mathrm{E}, 41.12282 \mathrm{~N}, 1000 \mathrm{~m}$, forest road in beech stand), Chas. $303(10 / 9 / 2009,22.26008$ E, 41.12827 N, $810 \mathrm{~m}$, forest road in Quercus frainetto stand), Chas. $320(10 / 9 / 2009,22.26185 \mathrm{E}, 41.12866 \mathrm{~N}, 790 \mathrm{~m}$, forest road in Quercus frainetto stand) - Sporadic. At forest roads in oak and beech stands, on siliceous substrates.

*Centaurea salonitana Vis. - H scap, EA - Chas. $1251(25 / 5 / 2011,22.2185$ E, $41.11962 \mathrm{~N}, 1120 \mathrm{~m}$, open thermophilous deciduous - Juniperus oxycedrus shrubland on rocky slope), Chas. 2229 (5/8/2012, 22.2215 E, 41.10976 N, 660 m, dry grassland) - Rare.

*Chondrilla juncea L. - H scap, ME - Chas. 200 (24/8/2009, 22.21799 E, 41.11178 N, $710 \mathrm{~m}$, forest road in thermophilous deciduous - Juniperus oxycedrus shrubland) Sporadic. At forest roads of lowland areas.

*Cichorium intybus L. - H scap, EA - Chas. 1576 (22/7/2011, 22.19377 E, 41.1178 N, $1020 \mathrm{~m}$, forest road in mixed beech - thermophilous deciduous stand) - Sporadic. At forest roads of lowland areas.

*Cirsium appendiculatum Griseb. - H scap, Bk - Chas. 1654 (23/8/2011, 22.2185 E, $41.16331 \mathrm{~N}, 1910 \mathrm{~m}$, tall-herb vegetation above treeline) - Common. In beech forests and above treeline, on damp ground. Between 1300 and $2100 \mathrm{~m}$.

*Cirsium arvense (L.) Scop. - H scap, EA - Chas. 112 (27/7/2009, 22.1844 E, 41.1335 $\mathrm{N}, 1190 \mathrm{~m}$, forest road in beech stand), Chas. $1667(31 / 8 / 2011,22.1911 \mathrm{E}$, $41.13253 \mathrm{~N}, 1370 \mathrm{~m}$, forest road in beech stand) - Sporadic at forest roads in beech and oak forests. Rare in mesic tall grasslands above treeline, up to $2100 \mathrm{~m}$.

*Cirsium candelabrum Griseb. - H bienn, Bk - Chas. 127 (6/8/2009, 22.18389 E, $41.15205 \mathrm{~N}, 1590 \mathrm{~m}$, forest road in beech stand) - Sporadic. At forest roads at treeline and in beech forests.

*Cirsium creticum (Lam.) d'Urv. subsp. creticum - H scap, Me - Chas. 1609 (18/8/2011, 22.21711 E, 41.13554 N, $1390 \mathrm{~m}$, ditch by roadside in beech stand) Sporadic. Ditches by roadsides and streambanks. Between 700 and $1400 \mathrm{~m}$.

*Cirsium eriophorum (L.) Scop. - H bienn, Eu - Chas. 187 (13/8/2009, 22.18661 E, $41.12282 \mathrm{~N}, 1000 \mathrm{~m}$, forest road in beech stand) - Common. At forest roads in beech stands and also in grasslands at treeline and above.

*Cirsium vulgare (Savi) Ten. - H bienn, ME - Chas. 1610 (18/8/2011, 22.19793 E, $41.11813 \mathrm{~N}, 1070 \mathrm{~m}$, forest road in mixed beech and thermophilous deciduous stand) - Sporadic. At roads in oak and beech forests.

*Crepis neglecta L. subsp. neglecta - T scap, BI - Chas. 1881 (24/5/2012, 22.22724 E, 41.11336 N, 760 m, phrygana), Chas. 1922 (30/5/2012, 22.19786 E, 41.10759 N, $760 \mathrm{~m}$, phrygana) - Common. Phrygana, grasslands and forest roads of lowland areas.

*Crepis sancta (L.) Bornm. - T scap, EA - Chas. 518 (2/5/2010, 22.22395 E, 41.11187 $\mathrm{N}, 680 \mathrm{~m}$, phrygana), Chas. 1110 (26/4/2011, $22.19913 \mathrm{E}, 41.10491 \mathrm{~N}, 680 \mathrm{~m}$, grassy opening in thermophilous deciduous and Juniperus oxycedrus shrubland) Common. In phrygana, grasslands and forest roads of lowland areas. 
*Crepis setosa Haller f. - T scap, EA - Chas. 1990 (4/6/2012, 22.25307 E, 41.10934 N, $630 \mathrm{~m}$, dry grassland) - Rare.

*Crepis vesicaria L. - H scap, Me - Chas. 1183 (16/5/2011, 22.21678 E, 41.11266 N, $750 \mathrm{~m}$, forest road in thermophilous deciduous - Juniperus oxycedrus shrubland), Chas. 2199 (27/7/2012, 22.25705 E, 41.1076 N, 610 m, dry grassland) - Rare.

Crepis viscidula Froel. subsp. viscidula - H scap, Bk - Chas. 1604 (13/8/2011, 22.21372 E, $41.15963 \mathrm{~N}, 2090 \mathrm{~m}$, grassy opening in subalpine shrubland), Chas. $2116(30 / 6 / 2012,22.21517 \mathrm{E}, 41.16317 \mathrm{~N}, 1980 \mathrm{~m}$, grassy opening in shrubland above treeline), MFG2: 579 - Sporadic. In subalpine grasslands and shrublands. Above $1800 \mathrm{~m}$.

*Crupina crupinastrum (Moris) Vis. - Tscap, EA - Chas. 1861 (16/5/2012, 22.22069 E, $41.10903 \mathrm{~N}, 640 \mathrm{~m}$, dry grassland) - Sporadic. In grasslands, phrygana and open shrublands of lowland areas.

*Crupina vulgaris Cass. - T scap, EA - Chas. 631 (19/5/2010, 22.21658 E, 41.11554 N, 920 m, phrygana), Chas. 1901 (24/5/2012, 22.22059 E, 41.1128 N, 720 m, open thermophilous deciduous - Juniperus oxycedrus shrubland) - Common. In grasslands, phrygana and open shrublands of lowland areas.

*Doronicum austriacum Jacq. - G rhiz, ME - Chas. 119 (6/8/2009, 22.18158 E, 41.1539 N, $1620 \mathrm{~m})$, Chas. 149 (6/8/2009, 22.19143 E, 41.14658 N, $1560 \mathrm{~m})$ Sporadic. In beech forests of mesic siliceous ground. Between 1350 and $1800 \mathrm{~m}$.

*Doronicum columnae Ten. - G rhiz, BC - Chas. 534 (8/5/2010, 22.20572 E, 41.13375 $\mathrm{N}, 1620 \mathrm{~m}$, beech stand), Chas. $1842(11 / 5 / 2012,22.18322 \mathrm{E}, 41.1506 \mathrm{~N}, 1550 \mathrm{~m}$, beech stand) - Common in beech stands at treeline. Rare in shrublands and streambanks above treeline.

Doronicum orientale Hoffm. - G rhiz, EA - Chas. 460 (17/4/2010, 22.22105 E, $41.11777 \mathrm{~N}, 840 \mathrm{~m}$, thermophilous deciduous stand), Chas. 1769 (12/4/2012, 22.18855 E, 41.11717 N, $950 \mathrm{~m}$, thermophilous deciduous stand), Voliotis 1983: 162 - Common. Beech, oak and thermophilous deciduous forests. $700-1500 \mathrm{~m}$.

Echinops bannaticus Schrad. - H scap, Bk - Chas. 1711 (24/9/2011, 22.23931 E, $41.12057 \mathrm{~N}, 860 \mathrm{~m}$, thermophilous deciduous stand), Chas. 2206 (27/7/2012, 22.25079 E, 41.13237 N, 1040 m, Quercus petraea stand), Chasapis et al. 2019 Sporadic. In thermophilous deciduous and oak forests.

*Echinops ritro L. - H scap, ES - Chas. 158 (13/8/2009, 22.2148 E, 41.11101 N, 730 $\mathrm{m}$, forest road in Juniperus oxycedrus - thermophilous deciduous shrubland), Chas. $952(14 / 8 / 2010,22.22454 \mathrm{E}, 41.11179 \mathrm{~N}, 670 \mathrm{~m}$, dry grassland), Chas. 958 (19/9/2010, 22.21669 E, 41.11167 N, 730 m, forest road in Juniperus oxycedrus thermophilous deciduous shrubland), Chas. 1704 (18/9/2011, 22.21597 E, 41.11166 $\mathrm{N}, 730 \mathrm{~m}$, opening with phrygana in Juniperus oxycedrus - thermophilous deciduous shrubland) - Common. At forest roads, dry grasslands, and phrygana. Between 600 and $900 \mathrm{~m}$.

*Echinops sphaerocephalus subsp. albidus (Boiss. \& Spruner) Kožuharov - H scap, BI - Chas. 951 (14/8/2010, 22.22454 E, 41.11179 N, 670 m, dry grassland), Chas. $2231(5 / 8 / 2012,22.22371 \mathrm{E}, 41.1116 \mathrm{~N}, 680 \mathrm{~m}$, forest road in dry grassland) Sporadic. At forest roads and dry grasslands of lowland areas.

*Erigeron acris L. - H bienn, ES - Chas. 388 (27/9/2009, 22.21237 E, 41.13016 N, 1320 m), Chas. 2222 (31/7/2012, 22.19165 E, 41.13416 N, 1440 m) - Sporadic. At roads in beech forests. 
*Erigeron annuus (L.) Desf. - T scap, [neotrp.] - Chas. $18(11 / 7 / 2009,22.18$ E, $41.12893 \mathrm{~N}, 1000 \mathrm{~m})$, Chas. $114(27 / 7 / 2009,22.1844 \mathrm{E}, 41.1335 \mathrm{~N}, 1190 \mathrm{~m})-$ Sporadic. At roads in beech forests.

*Erigeron canadensis L. - T scap/H bienn, [N-Am.] - Chas. 383 (27/9/2009, 22.21641 E, $41.1349 \mathrm{~N}, 1390 \mathrm{~m}$, forest road in beech stand) - Sporadic. At forest roads in beech, oak and thermophilous deciduous forests.

Erigeron glabratus Bluff \& Fingerh. - H, ME - Parent 2005: 222.

*Eupatorium cannabinum L. - H scap, Pt - Chas. 380 (27/9/2009, 22.2132 E, $41.14368 \mathrm{~N}, 1740 \mathrm{~m}$, forest road above treeline) - Sporadic. Streambanks in grasslands of lowland areas and ditches by roadsides.

*Filago arvensis L. - T scap, Pt - Chas. 5 (11/7/2009, 22.18029 E, 41.13137 N, 1060 $\mathrm{m}$, forest road in beech stand), Chas. 1948 (30/5/2012, 22.19495 E, $41.10944 \mathrm{~N}$, $760 \mathrm{~m}$, opening in shrubland of Juniperus oxycedrus and thermophilous deciduous), Chas. 1972 (4/6/2012, 22.20359 E, 41.11588 N, 980 m, bank of artificial lake), Chas. $2179(11 / 7 / 2012,22.18209 \mathrm{E}, 41.12662 \mathrm{~N}, 1020 \mathrm{~m}$, forest road in beech stand) - Common. in forest roads, dry grasslands and open shrublands of lowland areas.

*Filago germanica (L.) Huds. - T scap, Pt - Chas. 1981 (4/6/2012, 22.2531 E, $41.11268 \mathrm{~N}, 660 \mathrm{~m}$, dry grassland) - Sporadic. Roads and dry grasslands of lowland areas.

*Filago minima (Sm.) Pers. - T scap, Eu - Chas. 1933 (30/5/2012, 22.19717 E, $41.11148 \mathrm{~N}, 850 \mathrm{~m}$, open Juniperus oxycedrus - thermophilous deciduous shrubland) - Sporadic. At forest roads, dry grasslands and open shrublands of lowland areas.

Filago pyramidata L. - T scap, Me - Voliotis 1983: 162.

*Gnaphalium sylvaticum L. - H scap, ES - Chas. 108 (27/7/2009, 22.1803 E, 41.13128 N, 1060 m), Chas. 2227 (5/8/2012, 22.18804 E, 41.13563 N, 1370 m) - Sporadic. At forest roads in beech stands.

*Hieracium jankae R. Uechtr. - H scap, BA - Chas. 1725 (28/9/2011, 22.19094 E, 41.11757 N, $970 \mathrm{~m}$, thermophilous deciduous stand) - Sporadic. At forest roads in beech and thermophilous deciduous stands, also in thermophilous deciduous forests.

*Hieracium klisurae subsp. pseuderiopus (Zahn) Greuter - H scap, Bk - Chas. 106 $(27 / 7 / 2009,22.1844 \mathrm{E}, 41.1335 \mathrm{~N}, 1190 \mathrm{~m}$, forest road in beech stand) - Rare.

*Hieracium lachenalii Suter - H scap, EA - Chas. 2152 (6/7/2012, 22.25119 E, $41.12634 \mathrm{~N}, 850 \mathrm{~m}$, thermophilous deciduous stand), Chas. $2166(6 / 7 / 2012$, 22.26419 E, 41.13023 N, 820 m, Quercus frainetto stand), Chas. 2435 (19/6/2014, $22.29163 \mathrm{E}, 41.14721 \mathrm{~N}, 820 \mathrm{~m}$, oak forest) - Sporadic. In thermophilous deciduous stands and oak forests.

*Hieracium lazistanum subsp. leithneri (Boiss.) Greuter - H scap, BA - Chas. 736 (2/6/2010, 22.19803 E, 41.13173 N, 1610 m, open mixed stand of Pinus nigra with Juniperus communis on rocky ground at treeline), Chas. 2082 (25/6/2012, 22.20977 E, $41.14705 \mathrm{~N}, 1940 \mathrm{~m}$, open subalpine shrubland on stony ground), Chas. 2325 (26/6/2013, 22.23628 E, $41.16277 \mathrm{~N}, 1850 \mathrm{~m}$, sparse subalpine grassland), Chas. $2326(26 / 6 / 2013,22.22536 \mathrm{E}, 41.15675 \mathrm{~N}, 1780 \mathrm{~m}$, sparse subalpine grassland on stony ground), Chas. 2457 (14/7/2014, 22.22777 E, $41.1467 \mathrm{~N}, 1890 \mathrm{~m}$, rocky place above treeline) - Common. At stony and rocky places with sparse vegetation, at treeline and above. 
*Hieracium murorum L. - H scap, EA - Chas. 1196 (20/5/2011, 22.22484 E, 41.11828 $\mathrm{N}, 900 \mathrm{~m}$, rivulet in mixed beech - thermophilous deciduous stand), Chas. 1843 $(16 / 5 / 2012,22.20013 \mathrm{E}, 41.1206 \mathrm{~N}, 1140 \mathrm{~m}$, forest road in beech stand) - Common in beech forests. Sporadic in oak forests, thermophilous deciduous stands and forest roads in beech stands.

*Hieracium naegelianum Pančić subsp. naegelianum - H scap, Bk - Chas. 2368 $(10 / 7 / 2013,22.21304 \mathrm{E}, 41.15404 \mathrm{~N}, 1940 \mathrm{~m}$, sparse grassland on stony ground), Chas. $2418(30 / 7 / 2013,22.20396$ E, $41.15903 \mathrm{~N}, 1940 \mathrm{~m}$, sparse grassland on schistose scree) - Sporadic. Above treeline on screes and stony ground of schists with sparse vegetation.

Hieracium pannosum Boiss. subsp. pannosum - H scap, BA - Chas. 133 (6/8/2009, $22.18389 \mathrm{E}, 41.15205 \mathrm{~N}, 1590 \mathrm{~m}$, forest road in beech stand), Chas. 347 $(15 / 9 / 2009,22.25927 \mathrm{E}, 41.16312 \mathrm{~N}, 1760 \mathrm{~m}$, sparse grassland on stony ground), Chas. $2462(20 / 7 / 2014,22.2242 \mathrm{E}, 41.13322 \mathrm{~N}, 1520 \mathrm{~m}$, grassland on stony ground) MFG2: 619 - Common. Above and at treeline in open grasslands and shrublands on stony calcareous ground.

*Hieracium racemosum subsp. crinitum (Sm.) Rouy - H scap, BI - Chas. 325 (10/9/2009, 22.25007 E, 41.14596 N, 1480 m, beech stand), Chas. 945 (8/8/2010, 22.22373 E, $41.11547 \mathrm{~N}, 780 \mathrm{~m}$, thermophilous deciduous stand), Chas. 1670 (31/8/2011, 22.17096 E, 41.14581 N, 1560 m, beech stand), Chas. 1686 (10/9/2011, 22.26381 E, $41.15842 \mathrm{~N}, 1450 \mathrm{~m}$, beech stand), Chas. 1717 (24/9/2011, 22.24221 E, $41.11936 \mathrm{~N}, 790 \mathrm{~m}$, thermophilous deciduous - beech stand in stream), Chas. 1724 (28/9/2011, 22.19284 E, $41.11265 \mathrm{~N}, 750 \mathrm{~m}$, thermophilous deciduous stand) - Common in beech forests. Sporadic in oak and thermophilous deciduous stands on mesic ground.

*Hieracium sabaudum L. - H scap, EA - Chas. 1735 (19/10/2011, 22.18116 E, $41.13021 \mathrm{~N}, 1040 \mathrm{~m}$ ) - Sporadic. At forest roads and openings in beech stands.

*Hieracium schmidtii Tausch- H scap, EA - Chas. 737 (2/6/2010, 22.19803 E, $41.13173 \mathrm{~N}, 1610 \mathrm{~m}$, open shrubland of Pinus nigra and Juniperus communis subsp. nana on rocky ground at treeline), Chas. 2153 (6/7/2012, 22.25119 E, $41.12634 \mathrm{~N}, 850 \mathrm{~m}$, thermophilous deciduous stand), Chas. 2312 (18/6/2013, 22.2379 E, $41.12911 \mathrm{~N}, 1120 \mathrm{~m}$, thermophilous deciduous stand) - Sporadic. In thermophilous deciduous stands, oak forests and shrublands at treeline, on stony ground.

*Hieracium sericophyllum Nejčeff \& Zahn - H scap, BA - Chas. 2197 (15/7/2012, 22.23158 E, $41.15567 \mathrm{~N}, 1830 \mathrm{~m}$, subalpine grassland on stony ground) - Rare.

*Hieracium sparsum subsp. macedonicum (Boiss. \& Orph.) Zahn - Hscap, Bk - Chas. $2131(30 / 6 / 2012,22.21931 \mathrm{E}, 41.14675 \mathrm{~N}, 1780 \mathrm{~m}$, forest road above treeline), Chas. $2225(31 / 7 / 2012,22.17403 \mathrm{E}, 41.15523 \mathrm{~N}, 1910 \mathrm{~m}$, opening in subalpine shrubland) - Common. In subalpine grasslands and shrublands on stony siliceous ground.

*Hieracium sparsum subsp. schultzianum (Pančić \&Vis.) Zahn - Hscap, Bk - Chas. $1566(22 / 7 / 2011,22.17674 \mathrm{E}, 41.15212 \mathrm{~N}, 1760 \mathrm{~m}$, grassy opening in subalpine shrubland) - Rare.

*Hieracium sparsum Friv. subsp. sparsum - Hscap, BA - Chas. 913 (1/8/2010, 22.218 E, $41.16006 \mathrm{~N}, 1990 \mathrm{~m}$, subalpine grassland on stony ground), Chas. 1658 (23/8/2011, 22.21858 E, 41.16511 N, 1970 m, subalpine grassland) - Common. in subalpine grasslands and shrublands, on stony siliceous ground. 
Hieracium umbrosum Jord. - H scap, Eu - Chas. 2159 (6/7/2012, 22.26419 E, 41.13023 N, 820 m, Quercus frainetto stand), Chas. 2256 (1/6/2013, 22.27889 E, 41.13748 N, 820 m, Quercus frainetto stand), Chasapis et al. 2019 - Rare. In oak forests.

*Hypochaeris cretensis (L.) Bory \& Chaub. - H scap, Me - Chas. 1437 (22/6/2011, 22.20549 E, $41.11185 \mathrm{~N}, 830 \mathrm{~m}$, grassy opening in thermophilous deciduous stand), Chas. 1896 (24/5/2012, 22.20075 E, 41.11134 N, 870 m, phrygana with Juniperus oxycedrus) - Sporadic. In grasslands, phrygana and forest roads of lowland areas.

*Hypochaeris glabra L. - T scap, Me - Chas. 1936 (30/5/2012, 22.19717 E, 41.11148 $\mathrm{N}, 850 \mathrm{~m}$, open shrubland of Juniperus oxycedrus and thermophilous deciduous) Sporadic. In phrygana and open shrublands of lowland areas.

Hypochaeris maculata L. - H ros, ES - Chas. 1646 (23/8/2011, 22.21841 E, 41.16754 N, 2050 m), Chas. 2095 (25/6/2012, 22.21551 E, 41.14633 N, 1830 m), MFG2: 524 - Sporadic. In subalpine shrublands on siliceous substrates.

*Hypochaeris radicata L. - H scap, EA - Chas. 345 (15/9/2009, 22.27546 E, 41.14139 N, 920 m, forest road in Quercus frainetto stand), Chas. 1464 (27/6/2011, 22.20086 $\mathrm{E}, 41.11124 \mathrm{~N}, 850 \mathrm{~m}$, forest road in thermophilous deciduous stand) - Sporadic. At forest roads and dry grasslands of lowland areas.

*Inula conyzae (Griess.) DC. - H bienn, EA - Chas. 179 (13/8/2009, 22.18554 E, $41.12244 \mathrm{~N}, 950 \mathrm{~m}$, grassy opening in beech stand) - Sporadic. In forests and shrublands of lowland areas. Also in forest and shrubland openings.

*Inula ensifolia L. - H scap, EA - Chas. 929 (8/8/2010, 22.22752 E, 41.1294 N, 1540 m) - Sporadic. Grasslands at mid-altitudes up to the treeline, on stony calcareous ground.

Inula hirta L. - H scap, ES - Chas. 852 (26/6/2010, 22.22752 E, 41.12967 N, 1550 m, grassland), Chas. 1474 (27/6/2011, $22.2139 \mathrm{E}, 41.12225 \mathrm{~N}, 1090 \mathrm{~m}$, forest road in mixed beech and thermophilous deciduous stand), MFG2: 419 - Common. Grasslands at mid-altitudes up to the treeline and above, on stony ground. 1000 $1900 \mathrm{~m}$.

*Inula oculus-christi L. - H scap, EA - Chas. 351 (15/9/2009, 22.26424 E, 41.16013 $\mathrm{N}, 1540 \mathrm{~m}$, grassland), Chas. 2185 (11/7/2012, $22.19621 \mathrm{E}, 41.11576 \mathrm{~N}, 1020 \mathrm{~m}$, open thermophilous deciduous stand) - Sporadic. In dry grasslands, phrygana, open shrublands of Juniperus oxycedrus and open thermophilous deciduous forests on stony ground. Between 600 and $1600 \mathrm{~m}$.

*Inula salicina subsp. aspera (Poir.) Hayek - H scap, EA - Chas. 2038 (18/6/2012, $22.23793 \mathrm{E}, 41.12352 \mathrm{~N}, 990 \mathrm{~m}$, thermophilous deciduous stand), Chas. 2145 (6/7/2012, 22.2916 E, 41.14838 N, 830 m, grassland within Quercus frainetto stand) - Sporadic. In oak and thermophilous deciduous forests and forest openings.

*Inula verbascifolia subsp. aschersoniana (Janka) Tutin - H scap, BA - Chas. 192 (24/8/2009, 22.21624 E, 41.12382 N, 1070 m, open shrubland of Juniperus oxycedrus with thermophilous deciduous on rocky ground) - Common. At calcareous rocky places with sparse vegetation. Between 600 and $1300 \mathrm{~m}$.

*Jurinea mollis (L.) Rchb. - H scap, EA - Chas. 846 (26/6/2010, 22.21917 E, 41.1254 $\mathrm{N}, 1220 \mathrm{~m}$, open shrubland of Juniperus oxycedrus with thermophilous deciduous on rocky ground), Chas. $1863(16 / 5 / 2012,22.2163 \mathrm{E}, 41.11283 \mathrm{~N}, 770 \mathrm{~m}$, forest road in shrubland of Juniperus oxycedrus with thermophilous deciduous) Common. Rocky or stony places with sparse vegetation. Between 600 and $1900 \mathrm{~m}$. 
*Lactuca hispida DC. - H scap, EA - Chas. 2250 (1/6/2013, 22.27611 E, 41.14842 N, $1030 \mathrm{~m}$, streambank in beech stand) - Rare.

*Lactuca muralis (L.) Gaertn. - H scap, Pt - Chas. 36 (11/7/2009, 22.18 E, 41.12893 N, 1000 m), Chas. 1528 (12/7/2011, 22.21875 E, 41.13647 N, 1410 m) - Common. In mesic beech forests.

*Lactuca perennis L. - H scap, Eu - Chas. 2003 (8/6/2012, 22.21461 E, 41.11812 N, $1040 \mathrm{~m}$, thermophilous deciduous stand), Chas. 2351 (1/7/2013, 22.22358 E, $41.12988 \mathrm{~N}, 1470 \mathrm{~m}$, open beech - thermophilous deciduous stand) - Sporadic. In thermophilous deciduous and beech stands.

*Lactuca serriola L. - H bienn/T scap, Pt - Chas. 946 (14/8/2010, 22.1935 E, 41.10581 $\mathrm{N}, 640 \mathrm{~m}$, disturbed site in Platanus stand) - Rare. At disturbed sites, mainly forest roads, of lowland areas.

*Lactuca viminea (L.) J. Presl \& C. Presl - H bienn, Pt - Chas. 209 (24/8/2009, 22.2135 E, 41.1236 N, $1130 \mathrm{~m}$, forest road in beech stand) - Rare. At disturbed sites and open stony places of lowland areas.

*Lapsana communis subsp. adenophora (Boiss.) Rech. f. - H bienn, BA - Chas. 2230 (5/8/2012, 22.19019 E, $41.13981 \mathrm{~N}, 1500 \mathrm{~m}$, forest road in beech stand) - Sporadic. In beech and oak forests and also in forest openings and forest roads.

*Lapsana communis subsp. intermedia (M. Bieb.) Hayek - H bienn, - Chas. 1491 (2/7/2011, 22.27438 E, 41.13714 N, $880 \mathrm{~m}$, forest road in mixed oak - beech stand) - Sporadic. In beech and oak forests and also in forest openings and forest roads.

*Leontodon crispus subsp. asper (Waldst. \& Kit.) Rohlena - H ros, EA - Chas. 1407 (16/6/2011, 22.22675 E, $41.11381 \mathrm{~N}, 790 \mathrm{~m}$, phrygana), Chas. 1518 (7/7/2011, 22.22534 E, $41.11395 \mathrm{~N}, 760 \mathrm{~m}$, thermophilous deciduous stand) - Common at stony and rocky places with sparse vegetation all-over the mountain, mainly on calcareous substrates. Sporadic in thermophilous deciduous stands, shrublands of Juniperus oxycedrus and subalpine shrublands.

*Leontodon crispus Vill. subsp. crispus - H ros, Me - Chas. 2412 (30/7/2013, 22.19284 E, 41.1603 N, 1940 m, schistose scree with sparse vegetation) - Sporadic. Schistose screes and stony places with sparse vegetation on siliceous substrates.

Leontodon hispidus L. subsp. hispidus - H ros, Me - Chas. 131 (6/8/2009, 22.18389 E, $41.15205 \mathrm{~N}, 1590 \mathrm{~m}$, forest road in beech stand), Chas. $1648(23 / 8 / 2011,22.21864$ E, $41.16718 \mathrm{~N}, 2040 \mathrm{~m}$, subalpine grassland), Chas. 1649 (23/8/2011, $22.21864 \mathrm{E}$, $41.16718 \mathrm{~N}, 2040 \mathrm{~m}$, subalpine grassland), Chas. 2411 (30/7/2013, $22.27333 \mathrm{E}$, $41.13631 \mathrm{~N}, 1900 \mathrm{~m}$, schistose scree), Chas. 2452 (10/7/2014, $22.19968 \mathrm{E}$, 41.15446 N, 1770 m, schistose scree), Schuler 2004: tab. 20 (as Leontodon hispidus subsp. danubialis (Jacq.) Simonk.) - Sporadic. Schistose screes and stony places with sparse vegetation above treeline, subalpine grasslands and forest roads above $1200 \mathrm{~m}$.

*Onopordum acanthium L. - H bienn, Pt - Chas. 1674 (10/9/2011, 22.27333 E, $41.13631 \mathrm{~N}, 870 \mathrm{~m}$, forest road in oak stand) - Rare. At forest roads of lowland areas.

*Petasites hybridus (L.) G. Gaertn. et al. - H scap, EA - Chas. 533 (8/5/2010, 22.21201 E, $41.14339 \mathrm{~N}, 1740 \mathrm{~m}$, spring above treeline) - Common in stream and roads in beech forests. Sporadic in damp places at treeline.

*Picnomon acarna (L.) Cass. - T scap, Pt - Chas. 1673 (10/9/2011, 22.26907 E, 41.13039 N, 790 m, forest road in Quercus frainetto stand) - Rare. At forest roads and other disturbed sites of lowland areas. 
*Picris hieracioides subsp. spinulosa (Guss.) Arcang. - H scap/H bienn, Me - Chas. $299(10 / 9 / 2009,22.26094 \mathrm{E}, 41.13621 \mathrm{~N}, 980 \mathrm{~m}$, forest road in mixed oak and beech stand) - Sporadic. At roads in forests and shrublands of lowland areas.

*Picris pauciflora Willd. - T scap, EA - Chas. 1207 (20/5/2011, 22.22829 E, 41.1141 N, 800 m, phrygana), Chas. $1463(27 / 6 / 2011,22.20086 \mathrm{E}, 41.11124 \mathrm{~N}, 850 \mathrm{~m}$, forest road in thermophilous deciduous stand) - Sporadic. At forest roads, dry grasslands and phrygana of lowland areas.

Pilosella alpicola (Hoppe) F. W. Schultz \& Sch. Bip. - H scap, BC - Chas. 2465 (28/7/2014, 22.20836 E, $41.15946 \mathrm{~N}, 2170 \mathrm{~m}$, open subalpine grassland), MFG2: 606 - Rare, occasionally abundant. On siliceous above treeline ground. 1900 - 2180 $\mathrm{m}$.

*Pilosella bauhini subsp. magyarica (Peter) S. Bräut. - H scap, EA - Chas. 1229 (25/5/2011, 22.20097 E, 41.11133 N, 860 m, phrygana with Juniperus oxycedrus), Chas. $1479(27 / 6 / 2011,22.21645$ E, $41.12031 \mathrm{~N}, 1110 \mathrm{~m}$, thermophilous deciduous stand), Chas. 1889 (24/5/2012, 22.23659 E, 41.10786 N, 620 m, phrygana with Juniperus oxycedrus), Chas. 2074 (21/6/2012, 22.22206 E, 41.14625 N, 1760 m, grassy opening in subalpine shrubland) - Common. In grasslands, thermophilous deciduous forests and shrublands and oak forests. Mainly on stony ground between 600 and $1800 \mathrm{~m}$.

*Pilosella cf. calodon (Peter) Soják - H scap, EA - Chas. 1458 (22/6/2011, 22.21729 E, 41.11346 N, $780 \mathrm{~m}$, thermophilous deciduous stand) - Rare.

*Pilosella cymosa (L.) F. W. Schultz \& Sch. Bip. - H scap, ES - Chas. 2020 (13/6/2012, 22.22708 E, $41.12372 \mathrm{~N}, 1270 \mathrm{~m}$, thermophilous deciduous stand), Chas. $2422(26 / 5 / 2014,22.2734$ E, $41.1518 \mathrm{~N}, 1100 \mathrm{~m}$, grassy opening in beech stand) - Rare.

*Pilosella cymosa subsp. sabina (Sebast.) H. P. Fuchs - Hscap, EA - Chas. 1160 (6/5/2011, 22.27748 E, 41.14583 N, 980 m, Quercus frainetto stand), Chas. 1230 (25/5/2011, 22.21392 E, $41.12153 \mathrm{~N}, 1080 \mathrm{~m}$, open shrubland of Juniperus oxycedrus and thermophilous deciduous) - Common. In grasslands, thermophilous deciduous forests and shrublands and oak forests. Mainly on stony ground between 700 and $2100 \mathrm{~m}$.

Pilosella hypeurya (Peter) Soják - H scap, EA - Gottschlich \& Schuler in Greuter \& Raus 2011: 316.

*Pilosella leucopsilon (Arv.-Touv.) Gottschl. - H ros, EA - Chas. 79 (20/7/2009, 22.21272 E, $41.14376 \mathrm{~N}, 1750 \mathrm{~m}$, open subalpine shrubland), Chas. 1499 (2/7/2011, 22.27738 E, 41.14583 N, 980 m, Quercus frainetto stand), Chas. 1545 $(12 / 7 / 2011,22.23082 \mathrm{E}, 41.1536 \mathrm{~N}, 1860 \mathrm{~m}$, subalpine grassland on stony ground) - Common in grasslands and shrublands at and above treeline. Sporadic at mid- and lower altitudes.

*Pilosella macrotricha (Boiss.) F. W. Schultz \& Sch. Bip. - H scap, BA - Chas. 2037 (18/6/2012, 22.23847 E, 41.12092 N, 890 m, thermophilous deciduous stand) Rare.

*Pilosella onegensis Norrl. - H scap, ES - Chas. 2108 (30/6/2012, 22.19967 E, $41.11969 \mathrm{~N}, 1100 \mathrm{~m}$, forest road in mixed thermophilous deciduous - beech stand) - Rare.

*Pilosella pavichii (Heuff.) Arv.-Touv. - H scap, BA - Chas. 2107 (30/6/2012, $22.20444 \mathrm{E}, 41.12391 \mathrm{~N}, 1200 \mathrm{~m}$, forest road in beech stand), Chas. 2434 (19/6/2014, 22.29163 E, 41.14721 N, 820 m, oak forest), Chas. 2445 (6/7/2014, 
22.19463 E, $41.13006 \mathrm{~N}, 1470 \mathrm{~m}$, sparse grassland on rocky ground) - Sporadic. In forests and grasslands, on stony and rocky siliceous ground. Between 800 and 1600 m.

*Pilosella piloselloides (Vill.) Soják - H scap, EA - Chas. 344 (15/9/2009, 22.27546 E, $41.14139 \mathrm{~N}, 910 \mathrm{~m}$, forest road in mixed oak - beech stand), Chas. 2130 (30/6/2012, 22.22084 E, $41.14758 \mathrm{~N}, 1800 \mathrm{~m}$, forest road above treeline) Sporadic. At forest roads and stony open ground, between 600 and $1800 \mathrm{~m}$.

*Prenanthes purpurea L. - H scap, EA - Chas. 1552 (17/7/2011, 22.18557 E, 41.12405 $\mathrm{N}, 1000 \mathrm{~m})$ - Common. In mesic beech forests.

*Ptilostemon afer (Jacq.) Greuter subsp. afer - H bienn/ H scap, - Chas. 1608 $(18 / 8 / 2011,22.21214$ E, 41.14312 N, 1730 m) - Rare. At forest roads above 1400 m.

*Pulicaria dysenterica (L.) Bernh. - H scap, - Chas. 195 (24/8/2009, 22.21376 E, $41.12306 \mathrm{~N}, 1090 \mathrm{~m}$, forest road in beech stand), Chas. 309 (10/9/2009, 22.25447 E, $41.13756 \mathrm{~N}, 1210 \mathrm{~m}$, forest road in beech stand) - Sporadic. At forest roads in oak and beech forests of lowland areas, mainly in damp places.

*Scorzonera hispanica L. - H scap, ME - Chas. 937 (8/8/2010, 22.22729 E, 41.12913 $\mathrm{N}, 1540 \mathrm{~m}$, grassland), Chas. 1903 (24/5/2012, $22.23659 \mathrm{E}, 41.10786 \mathrm{~N}, 630 \mathrm{~m}$, phrygana and Juniperus oxycedrus) - Common in grasslands at mid-altitudes and at treeline. Sporadic in open shrublands of Juniperus oxycedrus and grasslands of lowland areas.

*Scorzonera mollis M. Bieb. - H scap, EA - Chas. $633(19 / 5 / 2010,22.21658$ E, 41.11554 N, 920 m, phrygana), Chas. 1137 (1/5/2011, 22.2164 E, 41.11356 N, 800 $\mathrm{m}$, phrygana) - Common. In phrygana and dry grasslands on stony or rocky, mainly calcareous, ground. $600-1500 \mathrm{~m}$.

Scorzoneroides cichoriacea (Ten.) Greuter - H ros, Me - Chas. 517 (2/5/2010, $22.21345 \mathrm{E}, 41.12108 \mathrm{~N}, 1070 \mathrm{~m}$, grassy opening in thermophilous deciduous stand), Chas. 1813 (2/5/2012, 22.21669 E, 41.1114 N, $730 \mathrm{~m}$, open shrubland of thermophilous deciduous and Juniperus oxycedrus), Voliotis 1983: 163 (as Leontodon cichoriaceaus (Ten.) Sanguin.) - Common. In stands and openings of thermophilous deciduous and grasslands of mid-altitudes up to the treeline. Between 600 and $1800 \mathrm{~m}$, mainly on calcareous substrates.

*Senecio rupestris Waldst. \& Kit. - H bienn/H scap, ME - Chas. 129 (6/8/2009, $22.18389 \mathrm{E}, 41.15205 \mathrm{~N}, 1590 \mathrm{~m}$, forest road in beech stand), Chas. 1572 (22/7/2011, 22.17379 E, 41.1554 N, 1920 m, subalpine shrubland) - Sporadic. At forest roads in beech forests and above treeline, in subalpine shrublands and grasslands on stony ground.

*Senecio vernalis Waldst. \& Kit. - T scap, EA - Chas. 451 (10/4/2010, 22.23015 E, $41.11602 \mathrm{~N}, 890 \mathrm{~m}$, phrygana), Chas. 1142 (1/5/2011, 22.22861 E, $41.11573 \mathrm{~N}, 900$ $\mathrm{m}$, phrygana) - Sporadic. In phrygana and dry grasslands of lowland areas, on stony ground.

*Senecio vulgaris L. - T scap, Pt - Chas. 995 (28/3/2011, 22.19232 E, 41.10976 N, 700 $\mathrm{m}$, forest road in thermophilous deciduous and Juniperus oxycedrus stand) - Rare. At forest roads and dry grasslands of lowland areas.

*Silybum marianum (L.) Gaertn. - Tscap, Me - Chas. obs. (1/6/2013, 22.26665 E, $41.12756 \mathrm{~N}, 700 \mathrm{~m}$, dry grassland) - Rare. At forest roads and dry grasslands of lowland areas. 
*Solidago virgaurea L. - H scap, Pt - Chas. 202 (24/8/2009, 22.21376 E, 41.12306 N, $1100 \mathrm{~m}$, forest road in beech stand), Chas. 1615 (18/8/2011, $22.21384 \mathrm{E}, 41.13748$ $\mathrm{N}, 1500 \mathrm{~m}$, forest road in beech stand) - Common in beech stands, at the lower part of beech forest zone, and by forest roads. Sporadic in oak forests and thermophilous deciduous stands.

*Sonchus asper subsp. glaucescens (Jord.) Ball - H bienn, Pt - Chas. 691 (2/6/2010, $22.18987 \mathrm{E}, 41.1253 \mathrm{~N}, 1190 \mathrm{~m}$, forest road in beech stand), Chas. 1176 $(11 / 5 / 2011,22.20048 \mathrm{E}, 41.12087 \mathrm{~N}, 1140 \mathrm{~m}$, forest road in beech stand) Sporadic. At roads in beech, oak and thermophilous deciduous forests.

*Tanacetum corymbosum (L.) Sch. Bip. - H scap, Me - Chas. 2025 (13/6/2012, 22.22857 E, 41.12158 N, 1190 m), Chas. 2028 (18/6/2012, 22.23719 E, 41.12441 $\mathrm{N}, 1050 \mathrm{~m})$ - Sporadic. In thermophilous deciduous stands on calcareous substrates.

Tanacetum macrophyllum (Waldst. \& Kit.) Sch. Bip. - H, BA - Parent 2005: 234.

*Tanacetum parthenium (L.) Sch. Bip. - H scap, EA - Chas. 20 (11/7/2009, 22.18 E, $41.12893 \mathrm{~N}, 1000 \mathrm{~m})$, Chas. $132(6 / 8 / 2009,22.18389 \mathrm{E}, 41.15205 \mathrm{~N}, 1590 \mathrm{~m})-$ Sporadic. In beech forests and forest roads.

*Taraxacum sect. Erythrocarpa Hand.-Mazz. - H ros - Chas. 1880 (19/5/2012, 22.22202 E, $41.14805 \mathrm{~N}, 1800 \mathrm{~m}$, subalpine grassland at disturbed ground due to overgrazing) - Sporadic. At forest roads and other disturbed sites.

*Taraxacum sect. Erythrosperma (H. Lindb.) Dahlst. - H ros - Chas. 1019 (8/4/2011, 22.23959 E, $41.11013 \mathrm{~N}, 660 \mathrm{~m}$, open shrubland of Juniperus oxycedrus and thermophilous deciduous), Chas. 2242 (11/4/2013, $22.24942 \mathrm{E}, 41.11244 \mathrm{~N}, 680 \mathrm{~m}$, open shrubland of Juniperus oxycedrus and thermophilous deciduous) - Common. In dry grasslands, open shrublands and forest roads of lowland areas.

*Taraxacum sect. Fontana Soest - H ros - Chas. 2365 (10/7/2013, 22.21497 E, 41.1529 N, 1920 m, above treeline fen) - Sporadic. Fens and other damp places at and above treeline.

Taraxacum sect. Ruderalia Kirschner et al. - H ros - Chas. 428 (14/11/2009, 22.22167 E, $41.1167 \mathrm{~N}, 840 \mathrm{~m}$, streambank in thermophilous deciduous stand), Chas. 1136 $(1 / 5 / 2011,22.20989 \mathrm{E}, 41.11183 \mathrm{~N}, 800 \mathrm{~m}$, grassy opening in thermophilous deciduous stand), Chas. $1310(2 / 6 / 2011,22.22503 \mathrm{E}, 41.14233 \mathrm{~N}, 1620 \mathrm{~m}$, streambank at treeline with grassy vegetation), Chas. 1819 (2/5/2012, 22.20288 E, $41.11145 \mathrm{~N}, 850 \mathrm{~m}$, forest road in thermophilous deciduous stand), Chas. 1852 $(16 / 5 / 2012,22.23615 \mathrm{E}, 41.1498 \mathrm{~N}, 1640 \mathrm{~m}$, grassland at treeline), Voliotis 1983: 163 (as T. officinale s.lat.) - Sporadic. Forest roads and other disturbed sites and grasslands, between 600 and $1900 \mathrm{~m}$.

* Taraxacum sect. Scariosa Hand.-Mazz. - H ros - Chas. 1853 (16/5/2012, 22.24159 E, $41.15127 \mathrm{~N}, 1750 \mathrm{~m}$, sparse grassland on stony ground) - Sporadic. On stony calcareous ground with sparse vegetation, at and above treeline.

*Telekia speciosa (Schreb.) Baumg. - H scap, EA - Chas. 863 (4/7/2010, 22.1769 E, 41.14644 N, $1460 \mathrm{~m}$, streambank in beech stand) - Rare. At streambanks in open beech stands and junctions of streams with forest roads in beech stands.

Tephroseris integrifolia subsp. aucheri (DC.) B. Nord. - H scap, BA - Chas. 650 $(24 / 5 / 2010,22.24492 \mathrm{E}, 41.15597 \mathrm{~N}, 1650 \mathrm{~m}$, grassy opening in beech stand at treeline), MFG2: 472 - Common. Grasslands and shrublands at and above treeline.

Tragopogon balcanicus Velen. - H bienn, Bk - Chas. 2271 (10/6/2013, 22.19258 E, $41.11796 \mathrm{~N}, 1030 \mathrm{~m}$, forest road in mixed thermophilous deciduous - beech stand), 
Voliotis 1983: 163 - Sporadic. At forest roads and stony places with sparse vegetation of lowland areas.

*Tragopogon dubius Scop. - T scap, EA - Chas. 1865 (16/5/2012, 22.20858 E, $41.11181 \mathrm{~N}, 810 \mathrm{~m}$, road in Pteridium thicket) - Rare.

*Tragopogon porrifolius L. - H bienn/T scap, Me- Chas. 1135 (1/5/2011, 22.22282 E, $41.11401 \mathrm{~N}, 780 \mathrm{~m}$, open Juniperus oxycedrus - thermophilous deciduous shrubland) - Rare.

*Tragopogon pratensis L. - H bienn, ES - Chas. 2032 (18/6/2012, 22.24048 E, $41.13032 \mathrm{~N}, 1220 \mathrm{~m}$, thermophilous deciduous stand) - Rare.

*Tragopogon samaritanii Boiss. - T scap, Me - Chas. 1243 (25/5/2011, 22.1878 E, $41.11842 \mathrm{~N}, 970 \mathrm{~m}$, forest road in mixed beech - thermophilous deciduous stand), Chas. $2105(30 / 6 / 2012,22.20444$ E, $41.12391 \mathrm{~N}, 1200 \mathrm{~m}$, forest road in beech stand) - Sporadic. At forest roads and stony places with sparse vegetation, at low and mid-altitudes.

*Tripleurospermum inodorum (L.) Sch. Bip. - T scap, Pt - Chas. 2175 (11/7/2012, 22.17897 E, 41.13198 N, 1060 m, forest road in beech stand) - Rare.

*Tripleurospermum tenuifolium (Kit.) Freyn - H bienn, EA - Chas. 1594 (13/8/2011, 22.22682 E, $41.11128 \mathrm{~N}, 670 \mathrm{~m}$, grassland on mesic ground) - Rare.

*Tussilago farfara L. - G rhiz/H scap, Pt - Chas. 466 (17/4/2010, 22.21376 E, $41.12306 \mathrm{~N}, 1100 \mathrm{~m}$, forest road in beech stand) - Common. At forest roads and eroded open places in beech forests and above treeline.

Willemetia stipitata (Jacq.) Dalla Torre - H ros, Eu - Chas. 1647 (23/8/2011, 22.21948 E, $41.1626 \mathrm{~N}, 1890 \mathrm{~m}$, Chas. 2139 (30/6/2012, $22.22149 \mathrm{E}, 41.15885 \mathrm{~N}, 1850 \mathrm{~m})$, MFG2: 575 (as Calycocorsus stipitatus (Jacq.) Rauschert), Schuler 2004: tab. 20 Sporadic. Above treeline fens.

*Xeranthemum annuum L. - T scap, EA - Chas. 9 (11/7/2009, 22.21633 E, 41.11148 $\mathrm{N}, 730 \mathrm{~m}$, forest road in Juniperus oxycedrus - thermophilous deciduous shrubland), Chas. 395 (9/10/2009, 22.22604 E, 41.11388 N, 780 m, phrygana), Chas. 2459 (20/7/2014, 22.22239 E, 41.1123 N, 700 m) - Sporadic. At forest roads, dry grasslands and phrygana of lowland areas.

*Xeranthemum cylindraceum Sm. - T scap, ME - Chas. 2380 (15/7/2013, 22.29215 E, 41.14569 N, 790 m, forest road in Quercus frainetto stand) - Rare.

\section{BETULACEAE}

*Alnus glutinosa (L.) Gaertn. subsp. glutinosa - P scap, ES - Chas. 1096 (26/4/2011, 22.19213 E, 41.11255 N, 720 m, mixed Alnus - Platanus - Salix stand on swampy place) - Rare.

*Betula pendula Roth - P scap, ES - Chas. 326 (15/9/2009, 22.27431 E, 41.15317 N, $1140 \mathrm{~m}$, forest road in beech stand) - Rare. In openings and forest roads of beech stands and also at treeline. Between 800 and $1700 \mathrm{~m}$.

Carpinus orientalis Mill. - P caesp/P scap, EA - Chas. 1945 (30/5/2012, 22.19362 E, $41.11032 \mathrm{~N}, 750 \mathrm{~m}$, thermophilous deciduous stand), FH1: map 50 - Common. Forms mixed stands with other thermophilous deciduous, with Quercus pubescens and Juniperus oxycedrus. Up to $1650 \mathrm{~m}$.

*Corylus avellana L. - P caesp, EA - Chas. 899 (1/8/2010, 22.19764 E, 41.1202 N, $1130 \mathrm{~m}$, mixed stand of the species with other deciduous) - Sporadic. Thermophilous deciduous forests and shrublands. 
*Corylus colurna L. - P scap, EA - Chas. 2352 (1/7/2013, 22.22418 E, 41.1298 N, $1500 \mathrm{~m}$, beech stand on calcareous scree) - Rare.

Ostrya carpinifolia Scop. - P scap/P caesp, MS - Chas. 33 (11/7/2009, 22.18 E, 41.12893 N, $1000 \mathrm{~m}$, beech stand), FH1: map 51, Parent 2005: 233 - Common. Forms mixed stands with thermophilous deciduous, oak and beech. Up to $1700 \mathrm{~m}$.

\section{BORAGINACEAE}

*Alkanna graeca Boiss. \& Spruner - H scap, Bk - Chas. 2276 (14/6/2013, 22.26197 E, $41.12875 \mathrm{~N}, 810 \mathrm{~m}$, forest road in Quercus frainetto stand) - Rare.

*Alkanna pindicola Hausskn. - H scap, Bk - Chas. 1105 (26/4/2011, 22.19913 E, $41.10491 \mathrm{~N}, 680 \mathrm{~m}$, grassy opening in shrubland thermophilous deciduous and Juniperus oxycedrus), Chas. 1143 (1/5/2011, 22.22861 E, 41.11573 N, 900 m, phrygana) - Common. In grasslands and phrygana of lowland areas.

Anchusa officinalis L. - H scap, EA - Chas. 741 (12/6/2010, 22.17575 E, 41.15354 N, $1820 \mathrm{~m}$, subalpine grassland), Chas. 2248 (1/6/2013, 22.26665 E, 41.12756 N, 720 m, dry grassland), Voliotis 1983: 162 - Sporadic. In forest roads and grasslands. Between 600 and $1850 \mathrm{~m}$.

*Anchusa undulata subsp. hybrida (Ten.) Bég. - H scap, Me - Chas. 1833 (11/5/2012, 22.21192 E, 41.11186 N, $790 \mathrm{~m}$, forest road in thermophilous deciduous stand) Rare.

*Buglossoides arvensis subsp. sibthorpiana (Griseb.) R. Fern. - T scap, EA - Chas. $510(2 / 5 / 2010,22.22395$ E, $41.11187 \mathrm{~N}, 680 \mathrm{~m}$, dry grassland), Chas. 1038 (8/4/2011, 22.23559 E, $41.11295 \mathrm{~N}, 700 \mathrm{~m}$, open shrubland of Juniperus oxycedrus) - Sporadic. In grasslands, phrygana and open shrublands of lowland areas.

*Buglossoides incrassata (Guss.) I. M. Johnst. subsp. incrassata - T scap, ME - Chas. 2014 (13/6/2012, 22.22812 E, 41.11712 N, 970 m, grassland) - Rare.

*Buglossoides purpurocaerulea (L.) I.M. Johnst. - H scap, EA - Chas. 1052 (16/4/2011, 22.24961 E, 41.13217 N, 1040 m, Quercus frainetto stand), Chas. 1215 (20/5/2011, 22.2297 E, 41.11926 N, $1050 \mathrm{~m}$, thermophilous deciduous stand) Common. In oak forests and thermophilous deciduous stands. $600-1300 \mathrm{~m}$.

Cerinthe minor subsp. auriculata (Ten.) Domac - H scap, EA - Chas. 635 (19/5/2010, 22.21622 E, 41.11562 N, 930 m, thermophilous deciduous and Juniperus oxycedrus shrubland), Voliotis 1983: 162 - Sporadic. In shrublands of lowland areas on calcareous substrates.

*Cynoglossum officinale L. - H bienn, ES - Chas. 2247 (1/6/2013, 22.26665 E, 41.12756 N, 720 m, dry grassland), Chas. 2264 (5/6/2013, 22.19684 E, 41.11893 N, $1110 \mathrm{~m}$, forest road in mixed beech - thermophilous deciduous stand), Chas. 2316 $(22 / 6 / 2013,22.19081 \mathrm{E}, 41.13441 \mathrm{~N}, 1430 \mathrm{~m}$, forest road in beech stand) Sporadic. In forest roads and dry grasslands. up to $1450 \mathrm{~m}$.

*Cynoglossum pustulatum subsp. parvifolium (Vis.) Sutorý - H bienn, Bk - Chas. 697 $(2 / 6 / 2010,22.19152 \mathrm{E}, 41.13046 \mathrm{~N}, 1350 \mathrm{~m}$, forest road in beech stand), Chas. 778 $(12 / 6 / 2010,22.1914 \mathrm{E}, 41.13055 \mathrm{~N}, 1350 \mathrm{~m}$, forest road in beech stand) - Rare.

*Echium italicum L. - H bienn, MS - Chas. 772 (12/6/2010, 22.21168 E, 41.11186 N, $790 \mathrm{~m}$, forest road in thermophilous deciduous stand) - Rare. At forest roads of lowland areas.

*Echium vulgare L. - H bienn, ES - Chas. 392 (27/9/2009, 22.21237 E, 41.13016 N, $1330 \mathrm{~m}$, forest road in beech stand), Chas. 712 (2/6/2010, 22.19349 E, $41.11518 \mathrm{~N}$, 
$910 \mathrm{~m}$, forest road in thermophilous deciduous stand) - Sporadic. At forest roads and dry grasslands of lowland areas.

Myosotis alpestris F. W. Schmidt subsp. alpestris - H scap, EA - Chas. 1307 (2/6/2011, 22.22014 E, $41.14262 \mathrm{~N}, 1610 \mathrm{~m})$, Chas. 1854 (16/5/2012, $22.23825 \mathrm{E}, 41.15154$ N, 1700 m), Chas. 1855 (16/5/2012, 22.22204 E, 41.14733 N, 1790 m), MFG2: 55 - Common. In subalpine grasslands and open shrublands.

* Myosotis alpestris subsp. suaveolens (Willd.) Strid - H scap, Bk - Chas. 539 (8/5/2010, 22.22281 E, 41.14959 N, 1750 m), Chas. 2318 (22/6/2013, 22.19441 E, $41.15519 \mathrm{~N}, 1780 \mathrm{~m})$ - Common. In subalpine grasslands and open shrublands.

*Myosotis arvensis (L.) Hill - T scap, ES - Chas. 1026 (8/4/2011, 22.19996 E, $41.10483 \mathrm{~N}, 670 \mathrm{~m}$, grassy opening in thermophilous deciduous and Juniperus oxycedrus shrubland), Chas. 1193 (20/5/2011, 22.22512 E, 41.11252 N, $690 \mathrm{~m}$, open shrubland of thermophilous deciduous and Juniperus oxycedrus) - Sporadic. In grasslands and shrublands of lowland areas.

Myosotis cadmea Boiss. - T scap, BA - Voliotis 1983: 162.

*Myosotis incrassata Guss. - T scap, ME - Chas. 453 (10/4/2010, 22.23015 E, $41.11602 \mathrm{~N}, 880 \mathrm{~m}$, phrygana), Chas. 1025 (8/4/2011, $22.23559 \mathrm{E}, 41.11295 \mathrm{~N}$, $700 \mathrm{~m}$, phrygana with Juniperus oxycedrus), Chas. 1081 (22/4/2011, 22.23431 E, $41.1205 \mathrm{~N}, 980 \mathrm{~m}$, grassy opening in thermophilous deciduous shrubland) Common. In dry grasslands and phrygana of lowland areas.

* Myosotis ramosissima Rochel subsp. ramosissima - $\mathrm{T}$ scap, EA - Chas. 497 $(25 / 4 / 2010,22.19838 \mathrm{E}, 41.10616 \mathrm{~N}, 710 \mathrm{~m}$, grassy opening in thermophilous deciduous and Juniperus oxycedrus shrubland), Chas. 1106 (26/4/2011, 22.19913 E, $41.10491 \mathrm{~N}, 670 \mathrm{~m}$, grassy opening in thermophilous deciduous and Juniperus oxycedrus shrubland) - Sporadic. In dry grasslands of lowland areas.

*Myosotis stricta Roem. \& Schult. - T scap, ES - Chas. 1306 (2/6/2011, 22.22255 E, $41.14599 \mathrm{~N}, 1770 \mathrm{~m})$ - Sporadic. In subalpine grasslands, on stony ground.

Myosotis sylvatica subsp. cyanea (Hayek) Vestergr. - H scap, Me - Chas. 597 (12/5/2010, 22.25182 E, 41.13869 N, 1280 m, beech stand), Chas. 659 (24/5/2010, 22.25993 E, 41.15629 N, $1400 \mathrm{~m}$, beech stand), Chas. 2067 (21/6/2012, 22.22215 E, $41.14733 \mathrm{~N}, 1790 \mathrm{~m}$, subalpine grassland), Voliotis 1983: 162 - Sporadic. In beech stands and grasslands at treeline and above. Up to $1950 \mathrm{~m}$.

Onosma heterophylla Griseb. - H scap, BA - Chas. 321 (10/9/2009, 22.26185 E, 41.12866 N, 790 m, forest road in Quercus frainetto stand), Chas. 636 (19/5/2010, $22.21658 \mathrm{E}, 41.11554 \mathrm{~N}, 920 \mathrm{~m}$, phrygana), Voliotis 1983: 162 (as O. echioides agg.), MFG2: 37 - Sporadic. In phrygana and open stony places, of lowland areas.

Pulmonaria rubra Schott - H scap, BC - Chas. 1016 (3/4/2011, 22.21539 E, 41.12417 N, 1080 m), Chas. 1775 (19/4/2012, 22.21483 E, 41.13191 N, 1300 m), Voliotis 1983: 162 - Common. Mesic beech stands.

*Symphytum bulbosum K. F. Schimp. - G rhiz, Me - Chas. 1815 (2/5/2012, 22.19105 E, $41.11721 \mathrm{~N}, 960 \mathrm{~m}$, forest road in mixed thermophilous deciduous - beech stand) - Rare.

*Symphytum ottomanum Friv. - G rhiz, BA - Chas. 2432 (19/6/2014, 22.27387 E, $41.1292 \mathrm{~N}, 710 \mathrm{~m})$ - Rare. In oak forests.

Symphytum tuberosum subsp. angustifolium (A. Kern.) Nyman - G rhiz, ME - Chas. $495(25 / 4 / 2010,22.18587$ E, 41.11929 N, 910 m), Chas. $526(2 / 5 / 2010,22.1895 \mathrm{E}$, $41.1216 \mathrm{~N}, 1070 \mathrm{~m})$, Chas. 1068 (16/4/2011, $22.2502 \mathrm{E}, 41.13668 \mathrm{~N}, 1250 \mathrm{~m})$, Voliotis 1983: 162 (as S. tuberosum subsp. nodosum (Schur) Soó), Parent 2005: 214 
- Common in mesic beech stands and Pteridium thickets. Sporadic at streams above treeline up to $1800 \mathrm{~m}$.

\section{BRASSICACEAE}

Aethionema saxatile subsp. graecum (Boiss. \&Spruner) Hayek - C suffr, BA - Chas. $449(10 / 4 / 2010,22.22918 \mathrm{E}, 41.12537 \mathrm{~N}, 1320 \mathrm{~m}$, rocky grassy opening in thermophilous deciduous and Juniperus oxycedrus shrubland), Chas. 498 $(25 / 4 / 2010,22.19838 \mathrm{E}, 41.10616 \mathrm{~N}, 720 \mathrm{~m}$, grassy opening in thermophilous deciduous and Juniperus oxycedrus shrubland), Chas. 595 (12/5/2010, 22.24486 E, $41.14047 \mathrm{~N}, 1480 \mathrm{~m}$, sparse grassland at stony slope), Voliotis 1983: 162 (as $A$. saxatile (L.) R.Br.) - Sporadic. At stony open places, mainly on calcareous substrates up to $1600 \mathrm{~m}$.

*Alliaria petiolata (M. Bieb.) Cavara \& Grande - H bienn, Pt - Chas. 151 (6/8/2009, 22.19143 E, 41.14649 N, 1560 m, beech stand) - Sporadic. In mesic beech stands, streambanks and Platanus stands.

*Alyssoides utriculata (L.) Medik. - C suffr, ME - Chas. 628 (19/5/2010, 22.21624 E, $41.11932 \mathrm{~N}, 1110 \mathrm{~m}$, open Juniperus oxycedrus and thermophilous deciduous shrubland) - Sporadic. On stony to rocky ground with sparse vegetation, $700-1600$ m.

*Alyssum alyssoides (L.) L. - T scap, Eu - Chas. 1113 (26/4/2011, 22.19856 E, 41.104 $\mathrm{N}, 640 \mathrm{~m}$, grassy opening in shrubland of thermophilous deciduous - Juniperus oxycedrus), Chas. 1222 (20/5/2011, 22.2296 E, 41.11862 N, 1030 m, opening with phrygana in thermophilous deciduous - Juniperus oxycedrus shrubland) - Common. Grasslands, phrygana and forest roads of lowland areas.

Alyssum chalcidicum Janka - H bienn, Bk - Chas. 247 (11/7/2009, 22.21102 E, $41.12743 \mathrm{~N}, 730 \mathrm{~m}$, forest road in thermophilous deciduous - Juniperus oxycedrus shrubland), FH2: map 1101 - Sporadic. In dry grasslands and forest roads of lowland areas.

Alyssum corymbosoides Formánek - H scap, Bk - FH2: 223.

*Alyssum doerfleri Degen - H caesp, Bk - Chas. 608 (19/5/2010, 22.21618 E, $41.11734 \mathrm{~N}, 1050 \mathrm{~m}$, rocky open place), Chas. 1252 (25/5/2011, 22.21696 E, $41.11933 \mathrm{~N}, 1130 \mathrm{~m}$ sparse grassland on rocky slope) - Sporadic. Rocky open places. $1000-1800 \mathrm{~m}$.

Alyssum montanum L. subsp. montanum - C suffr, EA - Chas. 2385 (20/7/2013, 22.23296 E, $41.14435 \mathrm{~N}, 2030 \mathrm{~m}$, sparse grassland on a steep slope), FH2: map 1087 - Sporadic. At stony open places above treeline.

*Alyssum montanum subsp. repens (Baumg.) Schmalh. - C suffr, ME - Chas. 669 $(24 / 5 / 2010,22.24675$ E, 41.15888 N, 1820 m), Chas. 1976 (4/6/2012, 22.23859 E, $41.11201 \mathrm{~N}, 680 \mathrm{~m}$, open Juniperus oxycedrus - thermophilous deciduous shrubland), Chas. 2289 (14/6/2013, 22.26275 E, 41.16245 N, $1700 \mathrm{~m}$, sparse grassland on stony ground) - Common. In grasslands and open shrublands on stony or rocky ground, between 650 and $2000 \mathrm{~m}$.

*Alyssum murale Waldst. \& Kit. - H scap, ME - Chas. 7 (28/8/2009, 22.21633 E, $41.11148 \mathrm{~N}, 1280 \mathrm{~m}$, forest road in beech stand), Chas. $2210(31 / 7 / 2012,22.17197$ E, $41.15213 \mathrm{~N}, 1920 \mathrm{~m}$, subalpine shrubland on rocky slope) - Sporadic. In grasslands and shrublands on rocky or stony ground, between 600 and $1950 \mathrm{~m}$.

Alyssum sibiricum Willd. - H scap, Bk - FH2: map 1111. 
*Alyssum turkestanicum Regel \& Schmalh - T scap, EA - Chas. 1035 (8/4/2011, 22.23555 E, 41.11448 N, 720 m, phrygana) - Sporadic. In grasslands, phrygana and forest roads of lowland areas.

*Arabidopsis thaliana (L.) Heynh. - T scap, Pt - Chas. 997 (28/3/2011, 22.20098 E, $41.11106 \mathrm{~N}, 850 \mathrm{~m}$, forest road in thermophilous deciduous stand), Chas. 1058 (16/4/2011, 22.26188 E, 41.13659 N, 990 m, beech stand) - Common. In various habitats of lowland areas.

Arabis alpina L. - H scap, ES - MFG1: 267.

*Arabis auriculata Lam. - T scap, ME - Chas. 2267 (10/6/2013, 22.18364 E, 41.13971 N, 1360 m, beech stand), Chas. 1526b (7/7/2011, 22.19458 E, 41.10565 N, $660 \mathrm{~m}$, thermophilous deciduous stand) - Sporadic. In various habitats up to treeline.

Arabis bryoides Boiss. - H caesp, Bk - Chas. 679 (24/5/2010, 22.24558 E, 41.15805 N, $1790 \mathrm{~m}$, sparse grassland on stony calcareous slope), MFG1: 266, FH2: map 1054, Schuler 2004: tab. 21 - Rare.

*Arabis collina Ten. - H scap, Me - Chas. 1138 (1/5/2011, 22.22767 E, 41.11958 N, $1080 \mathrm{~m}$, grassy opening in thermophilous deciduous - Juniperus oxycedrus shrubland), Chas. 1783 (22/4/2012, 22.23984 E, 41.13202 N, 1320 m, open thermophilous deciduous - Juniperus oxycedrus shrubland) - Sporadic. At stony places with sparse vegetation, between 700 and $1600 \mathrm{~m}$.

Arabis glabra (L.) Bernh. - H bienn, Eu - Chas. 656 (24/5/2010, 22.24492 E, 41.15597 $\mathrm{N}, 1640 \mathrm{~m}$, grassy opening in beech stand), Chas. $798(12 / 6 / 2010,22.18287 \mathrm{E}$, $41.14609 \mathrm{~N}, 1550 \mathrm{~m}$, opening in beech stand at treeline), Chas. 1227 (25/5/2011, 22.18864 E, $41.11817 \mathrm{~N}, 980 \mathrm{~m}$, forest road in beech and thermophilous deciduous stand), Voliotis 1983: 162, FH2: map 1044 - Sporadic. Forest openings, mainly in beech stands.

Arabis hirsuta L. - H bienn/ H scap, ME - Voliotis 1983: 162.

Arabis sagittata (Bertol.) DC. - H bienn, Eu - Chas. 799 (12/6/2010, 22.18287 E, $41.14609 \mathrm{~N}, 1550 \mathrm{~m}$, grassland at treeline), Chas. $1218(20 / 5 / 2011,22.22945 \mathrm{E}$, $41.11961 \mathrm{~N}, 1080 \mathrm{~m}$, thermophilous deciduous stand), Chas. 1877 (19/5/2012, $22.23103 \mathrm{E}, 41.15855 \mathrm{~N}, 1690 \mathrm{~m}$, subalpine grassland), FH2: map 1046 Common. Grasslands and forests, mainly thermophilous deciduous. $600-1900 \mathrm{~m}$.

*Arabis sudetica Tausch - H bienn/H scap, ME - Chas. 681 (24/5/2010, $22.25831 \mathrm{E}$, $41.15878 \mathrm{~N}, 1550 \mathrm{~m})$, Chas. 1304 (2/6/2011, 22.2249 E, $41.1426 \mathrm{~N}, 1620 \mathrm{~m})$, Chas. $1343(9 / 6 / 2011,22.2075 \mathrm{E}, 41.16035 \mathrm{~N}, 2150 \mathrm{~m})$ - Sporadic. In grasslands at treeline and above.

Arabis turrita L. - H scap, EA - Chas. 49 (11/7/2009, 22.18 E, 41.12893 N, 1000 m, beech stand), Chas. 658 (24/5/2010, $22.24492 \mathrm{E}, 41.15597 \mathrm{~N}, 1640 \mathrm{~m}$, beech stand at treeline), Voliotis 1983: 162, FH2: map 1051 - Common. In forests and shrublands all-over the mountain.

Aubrieta deltoidea (L.) DC. - C suffr, Me - Chas. 469 (17/4/2010, 22.21942 E, $41.11621 \mathrm{~N}, 930 \mathrm{~m})$, Chas. 626 (19/5/2010, $22.21658 \mathrm{E}, 41.11554 \mathrm{~N}, 920 \mathrm{~m})$, Voliotis 1983: 162 - Sporadic. On stony calcareous ground with phrygana or shrublands. Up to $1500 \mathrm{~m}$.

Barbarea bracteosa Guss. - H scap, Me - Chas. 748 (12/6/2010, 22.17392 E, 41.15513 N, $1910 \mathrm{~m}$, streambank above treeline), Chas. 1878 (19/5/2012, $22.22042 \mathrm{E}$, $41.16298 \mathrm{~N}, 1860 \mathrm{~m}$, streambank above treeline), FH2: 172 - Sporadic. In damp places above and at treeline. 
*Berteroa obliqua (Sm.) DC. - H scap, ME - Chas. 341 (15/9/2009, 22.27132 E, $41.15376 \mathrm{~N}, 1170 \mathrm{~m}$, forest road in beech stand) - Sporadic. In grasslands and forest roads up to $1200 \mathrm{~m}$.

*Calepina irregularis (Asso) Thell. - T scap, MS - Chas. 1009 (3/4/2011, 22.2099 E, $41.11156 \mathrm{~N}, 800 \mathrm{~m}$, grassland within thermophilous deciduous - Juniperus oxycedrus shrubland), Chas. 1812 (2/5/2012, 22.21669 E, 41.1114 N, $720 \mathrm{~m}$, opening with phrygana in thermophilous deciduous - Juniperus oxycedrus shrubland) - Sporadic. Grasslands, open shrublands and forest roads of lowland areas.

*Camelina microcarpa DC. - T scap, MS - Chas. 1134 (1/5/2011, 22.21572 E, $41.11228 \mathrm{~N}, 760 \mathrm{~m}$, forest road in shrubland of thermophilous deciduous and Juniperus oxycedrus), Chas. 1257 (25/5/2011, 22.21837 E, 41.1198 N, 1140 m, open shrubland of thermophilous deciduous and Juniperus oxycedrus) - Sporadic. In grasslands, open shrublands and roads of lowland areas.

*Capsella bursa-pastoris (L.) Medik. subsp. bursa-pastoris - $\mathrm{T}$ scap/H bienn, Co Chas. $553(8 / 5 / 2010,22.22256 \mathrm{E}, 41.15013 \mathrm{~N}, 1750 \mathrm{~m}$, subalpine grassland) Common. In grasslands and roads, up to $1950 \mathrm{~m}$.

Cardamine acris Griseb. - H scap, Bk - Chas. 761 (12/6/2010, 22.17555 E, 41.15651 $\mathrm{N}, 1860 \mathrm{~m}$, stream above treeline), Chas. 1317 (2/6/2011, 22.21895 E, $41.14233 \mathrm{~N}$, $1610 \mathrm{~m}$, fen at treeline), Voliotis 1983: 162 (as C. raphanifolia subsp. acris (Griseb.) Schulz), MFG1: 257 (as C. raphanifolia subsp. acris (Griseb.) Schulz), FH2: map 1034, Schuler 2004: tab. 20 - Common in damp places, mainly fens, at and above treeline. Sporadic in damp places by roadsides in beech stands. 1500 $2100 \mathrm{~m}$.

Cardamine bulbifera (L.) Crantz - G rhiz, Eu - Chas. 43 (11/7/2009, 22.18 E, 41.12893 $\mathrm{N}, 1000 \mathrm{~m}$, beech stand), Chas. 152 (6/8/2009, $22.19142 \mathrm{E}, 41.14685 \mathrm{~N}, 1560 \mathrm{~m}$, beech stand at treeline), Chas. $1824(2 / 5 / 2012,22.21462 \mathrm{E}, 41.13506 \mathrm{~N}, 1430 \mathrm{~m}$, beech stand), Voliotis 1983: 162 (as Dentaria bulbifera L.), FH2: map 1030 Common. In mesic beech stands and Pteridium thickets of mid-altitudes up to the treeline.

*Cardamine glauca DC. - T scap, BI, - Chas. 2266 (10/6/2013, 22.17108 E, 41.14554 $\mathrm{N}, 1540 \mathrm{~m}$, beech stand) - Rare.

*Cardamine graeca L. - T scap, Me - Chas. 459 (17/4/2010, 22.22105 E, 41.11777 N, $840 \mathrm{~m}$, thermophilous deciduous stand), Chas. 1018 (8/4/2011, 22.24168 E, $41.11214 \mathrm{~N}, 680 \mathrm{~m}$, thermophilous deciduous stand) - Common. In thermophilous deciduous stands and oak forests.

*Cardamine hirsuta L. - T scap, Co - Chas. 996 (28/3/2011, 22.20098 E, 41.11106 N, $850 \mathrm{~m}$, forest road in thermophilous deciduous stand), Chas. 1059 (16/4/2011, 22.26383 E, $41.13482 \mathrm{~N}, 920 \mathrm{~m}$, stream in beech stand) - Common. In various habitats of lowland areas.

Cardamine impatiens L. - T scap, ES - Chas. 695 (2/6/2010, 22.19095 E, 41.12946 N, $1290 \mathrm{~m}$, forest road in beech stand), Chas. 1942 (30/5/2012, 22.19175 E, 41.11275 N, 720 m, alder stand), Chas. 2169 (6/7/2012, 22.27755 E, $41.1525 \mathrm{~N}, 1100 \mathrm{~m}$, forest road in beech stand), Chas. 2268 (10/6/2013, 22.18238 E, 41.14194 N, 1360 $\mathrm{m}$, forest road in beech stand), Kučera et al. 2006: 190 - Sporadic. Mesic grounds in forest roads and beech stands (open places).

*Clypeola jonthlaspi L. subsp. jonthlaspi - T scap, MS - Chas. 1112 (26/4/2011, $22.19913 \mathrm{E}, 41.10491 \mathrm{~N}, 680 \mathrm{~m}$, grassy opening in shrubland of thermophilous 
deciduous and Juniperus oxycedrus), Chas. 1141 (1/5/2011, 22.21634 E, 41.11581 N, 940 m, open shrubland of thermophilous deciduous and Juniperus oxycedrus) Common. Grasslands, open shrublands and phrygana of lowland areas.

*Diplotaxis tenuifolia (L.) DC. - H scap, EA - Chas. 211 (24/8/2009, 22.21376 E, $41.12306 \mathrm{~N}, 1100 \mathrm{~m}$, forest road in beech stand with thermophilous deciduous), Chas. $1471(27 / 6 / 2011,22.21725$ E, 41.11294 N, 770 m, forest road in shrubland of thermophilous deciduous and Juniperus oxycedrus) - Sporadic. In forest roads of lowland areas.

Draba lasiocarpa Rochel - H ros, BC - Chas. 443 (10/4/2010, 22.23015 E, 41.11602 $\mathrm{N}, 890 \mathrm{~m}$, sparse phrygana on stony slope), Chas. 547 (8/5/2010, 22.23426 E, $41.15355 \mathrm{~N}, 1850 \mathrm{~m}$, subalpine sparse grassland on stony slope), Voliotis 1983: 163 (as D. athoa (Griseb.) Boiss.), FH2: map 1140 - Common. On stony and rocky places with sparse vegetation, between 850 and $2150 \mathrm{~m}$.

*Draba verna (L.) Chevall. - T scap, EA - Chas. 979 (13/3/2011, 22.25311 E, 41.11691 N, 720 m), Chas. 1820 (2/5/2012, 22.22206 E, 41.14625 N, 1780 m) - Common. In grasslands between 600 and $1800 \mathrm{~m}$.

Erysimum comatum Pančić - H bienn, Bk - Chas. 591 (12/5/2010, 22.24484 E, $41.1411 \mathrm{~N}, 1510 \mathrm{~m}$, sparse grassland), Chas. 1129 (1/5/2011, 22.22112 E, 41.11516 N, 850 m, phrygana), Chas. 2288 (14/6/2013, 22.26275 E, 41.16245 N, $1700 \mathrm{~m}$, subalpine grassland), FH2: map 983 - Common. In sparse grasslands on stony calcareous ground, between 800 and $2050 \mathrm{~m}$.

Erysimum crassistylum C. Presl - H bienn, BI - Chas. 1277 (28/5/2011, 22.21251 E, $41.11196 \mathrm{~N}, 790 \mathrm{~m}$, forest road in shrubland of thermophilous deciduous and Juniperus oxycedrus), Chas. 1301 (2/6/2011, 22.21512 E, 41.11245 N, 780 m, forest road in shrubland of thermophilous deciduous and Juniperus oxycedrus), Chas. $1918(24 / 5 / 2012,22.22723$ E, $41.11336 \mathrm{~N}, 760 \mathrm{~m}$, phrygana on stony ground), FH2: map 970 - Sporadic. At forest roads and open shrublands of lowland areas.

*Erysimum cuspidatum (M. Bieb.) DC. - H bienn, EA - Chas. 609 (19/5/2010, 22.2155 E, $41.11597 \mathrm{~N}, 980 \mathrm{~m}$, thermophilous deciduous stand), Chas. 1420 (16/6/2011, 22.22695 E, 41.11534 N, 880 m, phrygana with Juniperus oxycedrus), Chas. $2000(8 / 6 / 2012,22.218 \mathrm{E}, 41.1161 \mathrm{~N}, 920 \mathrm{~m}$, thermophilous deciduous stand) - Sporadic. In open thermophilous deciduous stands and Juniperus oxycedrus shrublands, between 700 and $1200 \mathrm{~m}$.

*Erysimum microstylum Hausskn. - H scap, Bk - Chas. 2279 (14/6/2013, 22.2687 E, $41.16272 \mathrm{~N}, 1560 \mathrm{~m}$, grassland on stony ground) - Rare.

*Hesperis Iaciniata All. subsp. laciniata - H scap/H bienn, Me - Chas. 464 (17/4/2010, $22.22295 \mathrm{E}, 41.11357 \mathrm{~N}, 750 \mathrm{~m}$, phrygana on stony ground), Chas. 1086 $(22 / 4 / 2011,22.23452 \mathrm{E}, 41.12167 \mathrm{~N}, 1020 \mathrm{~m}$, grassy opening in thermophilous deciduous stand) - Sporadic. In grasslands and phrygana of lowland areas, on stony ground.

*Hornungia petraea (L.) Rchb. - T scap, Me - Chas. 2240 (11/4/2013, 22.24975 E, $41.11326 \mathrm{~N}, 680 \mathrm{~m}$ ) - Sporadic. Grassy openings of thermophilous deciduous stands.

Iberis saxatilis L. - C suffr, Me - Chas. 589 (12/5/2010, 22.24717 E, 41.14303 N, 1610 $\mathrm{m}$, sparse grassland on stony ground), Chas. 1320 (2/6/2011, $22.2263 \mathrm{E}, 41.13515$ $\mathrm{N}, 1640 \mathrm{~m}$, sparse grassland on stony ground at treeline), MFG1: 333 - Sporadic. In 
grasslands, open shrublands and screes at treeline and above, on calcareous substrates.

Iberis sempervirens L. - C suffr, EA - Chas. 562 (8/5/2010, 22.2315 E, $41.16333 \mathrm{~N}$, $1820 \mathrm{~m}$, subalpine shrubland), Chas. 590 (12/5/2010, $22.24717 \mathrm{E}, 41.14303 \mathrm{~N}$, $1610 \mathrm{~m}$, sparse grassland on stony ground), MFG1: 334 - Common. In open shrublands and grasslands at treeline and above, on calcareous substrates.

*Isatis tinctoria L. subsp. tinctoria - H bienn, EA - Chas. 606 (19/5/2010, 22.21718 E, $41.11969 \mathrm{~N}, 1140 \mathrm{~m}$, open shrubland of thermophilous deciduous and Juniperus oxycedrus), Chas. 1253 (25/5/2011, 22.2185 E, $41.11962 \mathrm{~N}, 1120 \mathrm{~m}$, open shrubland thermophilous deciduous and Juniperus oxycedrus) - Sporadic. At openings of thermophilous deciduous shrublands and forests.

*Lepidium campestre (L.) W. T. Aiton - T scap, EA - Chas. 1380 (9/6/2011, 22.19249 E, $41.11661 \mathrm{~N}, 940 \mathrm{~m}$, forest road in thermophilous deciduous stand) - Rare.

*Lunaria annua subsp. pachyrhiza (Borbás) Hayek - H bienn, BI - Chas. 463 $(17 / 4 / 2010,22.21966$ E, $41.11622 \mathrm{~N}, 920 \mathrm{~m})$ - Sporadic. In thermophilous deciduous stands.

Matthiola fruticulosa subsp. valesiaca (Gaudin) P. W. Ball - C suffr, ME - Chas. 594 (12/5/2010, 22.24717 E, 41.14303 N, 1610 m), Voliotis 1983: 163, FH2: map 1013 - Common. Sparse grasslands on stony calcareous ground. 1400 - $2000 \mathrm{~m}$.

*Microthlaspi perfoliatum (L.) F. K. Mey. - T scap, Pt - Chas. 983 (23/3/2011, 22.22899 E, $41.11933 \mathrm{~N}, 1070 \mathrm{~m}$, grassy clearing in thermophilous deciduous stand) - Common. In grasslands and phrygana of lowland areas.

*Noccaea lutescens F. K. Mey.- H scap, Bk - Chas. 546 (8/5/2010, 22.22281 E, 41.14959 N, $1750 \mathrm{~m})$, Chas. 1181 (16/5/2011, 22.22394 E, $41.15186 \mathrm{~N}, 1780 \mathrm{~m})-$ Common. In subalpine grasslands, between 1600 and $2180 \mathrm{~m}$.

*Noccaea tymphaea (Hausskn.) F. K. Mey. - H scap, Bk - Chas. 1342 (9/6/2011, 22.20318 E, 41.16154 N, 2100 m, subalpine grassland) - Rare.

?Noccaea praecox (Wulfen) F. K. Mey. - Hscap, BI - Voliotis 1983: 163.

*Rorippa amphibia (L.) Besser - Hscap, ES - Chas. 2262 (5/6/2013, 22.20927 E, 41.12597 N, 1210 m, swamp in beech stand) - Rare.

*Rorippa sylvestris (L.) Besser - Hscap, EA - Chas. 1639 (23/8/2011, 22.2021 E, $41.12252 \mathrm{~N}, 1180 \mathrm{~m}$, forest road in beech stand) - Rare.

*Rorippa thracica (Griseb.) Fritsch - Hscap, BA - Chas. 574 (12/5/2010, 22.25085 E, 41.13039 N, 950 m, open Juniperus oxycedrus shrubland within Quercus frainetto stand), Chas. $692(2 / 6 / 2010,22.18987 \mathrm{E}, 41.1253 \mathrm{~N}, 1190 \mathrm{~m}$, forest road in beech stand), Chas. 1941 (30/5/2012, 22.19278 E, 41.1149 N, 880 m, open shrubland of thermophilous deciduous and Juniperus oxycedrus) - Common. Grasslands and shrublands up to $1400 \mathrm{~m}$.

*Sisymbrium officinale (L.) Scop. - Tscap, ES/[Co] - Chas. 1964 (4/6/2012, 22.25445 E, $41.10684 \mathrm{~N}, 600 \mathrm{~m}$, dry grassland) - Rare.

*Teesdalia coronopifolia (J. P. Bergeret) Thell. - Tscap, Me - Chas. 1048 (11/4/2011, $22.19325 \mathrm{E}, 41.11518 \mathrm{~N}, 910 \mathrm{~m}$, forest road in thermophilous deciduous stand) Rare.

CAMPANULACEAE

Asyneuma limonifolium (L.) Janch. - Hscap, Me - Chas. 812 (26/6/2010, 22.22359 E, $41.11637 \mathrm{~N}, 790 \mathrm{~m}$, open thermophilous deciduous shrubland on stony ground), 
MFG2: 390 - Common. On stony and rocky calcareous ground with sparse vegetation all-over the mountain.

*Asyneuma pichleri (Vis.) D. Lakušić \& F. Conti - H scap, Bk - Chas. 1578 $(22 / 7 / 2011,22.17723$ E, $41.15377 \mathrm{~N}, 1750 \mathrm{~m})$ - Sporadic. In beech stands at treeline.

*Campanula bononiensis L. - H scap, ES - Chas. 1641 (23/8/2011, 22.19764 E, $41.12011 \mathrm{~N}, 1130 \mathrm{~m}$, road embankment in beech stand), Chas. 2172 (11/7/2012, 22.19222 E, 41.11786 N, $1000 \mathrm{~m}$, road embankment in mixed beech stand and thermophilous deciduous) - Rare.

*Campanula cervicaria L. - H scap, ES - Chas. 71 (20/7/2009, 22.21484 E, 41.1401 $\mathrm{N}, 1570 \mathrm{~m}$, grassy opening in beech stand), Chas. $1562(22 / 7 / 2011,22.17674 \mathrm{E}$, $41.15203 \mathrm{~N}, 1750 \mathrm{~m}$, subalpine grassland), Chas. 2055 (21/6/2012, 22.21706 E, $41.14185 \mathrm{~N}, 1590 \mathrm{~m}$, streambank at treeline) - Sporadic. Beech forests, grasslands within beech stands and above treeline.

*Campanula foliosa Ten. - H scap, BI - Chas. 1579 (22/7/2011, 22.17723 E, 41.15377 N, 1750 m, beech stand), Chas. 2054 (21/6/2012, 22.21706 E, 41.14185 N, 1590 m, grassland) - Sporadic. In beech stands and grasslands at treeline.

Campanula formanekiana Degen \& Dörfl. - H scap, Bk - Chas. 821 (26/6/2010, 22.22203 E, 41.1167 N, 840 m), Voliotis 1983: 163, MFG2: 378 - Sporadic. Shady places on calcareous rocks in different vegetation types. $700-1600 \mathrm{~m}$.

Campanula glomerata L. - H scap, EA - Chas. 359 (15/9/2009, 22.25928 E, 41.16285 N, 1740 m), Chas. 366 (15/9/2009, 22.26094 E, 41.16315 N, 1770 m), Chas. 2372 $(15 / 7 / 2013,22.25439$ E, 41.16296 N, 1840 m), MFG2: 382 - Common. In grasslands from $1300 \mathrm{~m}$ up to $1950 \mathrm{~m}$.

*Campanula lingulata Waldst. \& Kit. - H bienn, Bk - Chas. 1206 (20/5/2011, 22.22876 E, 41.11456 N, 820 m, phrygana), Chas. 1924 (30/5/2012, 22.19705 E, $41.10713 \mathrm{~N}, 740 \mathrm{~m}$, open Juniperus oxycedrus shrubland) - Common. in open shrublands and phrygana of lowland areas.

*Campanula persicifolia L. - H scap, ES - Chas. 100 (27/7/2009, 22.1844 E, 41.1335 N, 1190 m, beech stand), Chas. 289 (10/9/2009, $22.25007 \mathrm{E}, 41.14596 \mathrm{~N}, 1470 \mathrm{~m}$, beech stand), Chas. 358 (15/9/2009, 22.25928 E, 41.16285 N, 1740 m, subalpine grassland) - Common in oak and beech forests. Sporadic in subalpine grasslands.

*Campanula scutellata Griseb. - T scap, Bk - Chas. 898 (1/8/2010, 22.20909 E, 41.12839 N, $1280 \mathrm{~m}$, forest road in beech stand), Chas. 2275 (14/6/2013, 22.26197 E, 41.12875 N, 800 m, forest road in Quercus frainetto stand) - Rare.

*Campanula sparsa Friv. - T scap, Bk - Chas. 28 (11/7/2009, 22.18 E, 41.12893 N, $1000 \mathrm{~m}$, beech stand), Chas. 97 (27/7/2009, $22.1844 \mathrm{E}, 41.1335 \mathrm{~N}, 1190 \mathrm{~m}$, forest road in beech stand), Chas. $823(26 / 6 / 2010,22.22262 \mathrm{E}, 41.11671 \mathrm{~N}, 820 \mathrm{~m}$, thermophilous deciduous stand), Chas. 868 (4/7/2010, $22.22214 \mathrm{E}, 41.11716 \mathrm{~N}$, $830 \mathrm{~m}$, thermophilous deciduous stand) - Common. Forests and shrublands up to $1500 \mathrm{~m}$.

Campanula spatulata Sm. - G bulb, Bk - Chas. 739 (12/6/2010, 22.17429 E, 41.15442 $\mathrm{N}, 1890 \mathrm{~m}$, grassy opening in subalpine shrubland), Chas. $1228(25 / 5 / 2011$, $22.20097 \mathrm{E}, 41.11133 \mathrm{~N}, 860 \mathrm{~m}$, grassy opening in thermophilous deciduous stand), MFG2: 373 - Common. In forests, shrublands and grasslands all-over the mountain. 
*Campanula trachelium L. - H scap, EA - Chas. 98 (27/7/2009, 22.1844 E, 41.1335 N, 1190 m, beech stand), Chas. 304 (10/9/2009, 22.25447 E, 41.13756 N, $1210 \mathrm{~m}$, forest road in beech stand) - Common in beech and oak forests and forest roads.

Campanula velebitica L. - H scap, Bk - Chas. 77 (20/7/2009, 22.2201 E, 41.16162 N, 1900 m, subalpine shrubland), Chas. 138 (6/8/2009, 22.19142 E, 41.14685 N, 1570 $\mathrm{m}$, shrubland of Juniperus communis at treeline), Chas. 921 (1/8/2010, $22.21724 \mathrm{E}$, 41.16149 N, $2010 \mathrm{~m}$, subalpine shrubland), Strid \& Papanicolaou 1981: 77 Common in subalpine shrublands. Sporadic in subalpine grasslands.

*Campanula versicolor Andrews - H scap, BI - Chas. 930 (8/8/2010, 22.22126 E, $41.12318 \mathrm{~N}, 1000 \mathrm{~m}$, open thermophilous deciduous stand on stony ground) Sporadic. On calcareous rocks in different vegetation types up to $1500 \mathrm{~m}$.

Edraianthus graminifolius (L.) A. DC. - C suffr, BI - Chas. 837 (26/6/2010, 22.21917 E, $41.1254 \mathrm{~N}, 1230 \mathrm{~m}$, open shrubland of thermophilous deciduous and Juniperus oxycedrus on rocky ground), Chas. 920 (1/8/2010, 22.21796 E, 41.1615 N, 1970 m, rocky opening in subalpine shrubland), MFG2: 395, Schuler 2004: tab. 21 Common at stony or rocky places above treeline. Occasionally descending to the forest zone.

*Jasione heldreichii Boiss. \& Orph. - H bienn, BA - Chas. 170 (13/8/2009, 22.18554 E, $41.12244 \mathrm{~N}, 950 \mathrm{~m}$, rocky grassy opening in beech stand), Chas. 2120 $(30 / 6 / 2012,22.19324 \mathrm{E}, 41.11536 \mathrm{~N}, 910 \mathrm{~m}$, forest road in thermophilous deciduous stand) - Sporadic. At stony open places up to the treeline.

Jasione orbiculata Velen. - H scap, BI - Chas. 911 (1/8/2010, 22.21796 E, 41.1615 N, $1970 \mathrm{~m}$, rocky opening in subalpine shrubland), MFG2: 396 (as J. laevis subsp. orbiculata (Velen.) Tutin) - Sporadic. At stony and rocky places above treeline.

Legousia speculum-veneris (L.) Chaix - Tscap, ME - Chas. obs. (26/5/2014, 22.28499 E, $41.14117 \mathrm{~N}, 760 \mathrm{~m})$, Voliotis 1983: 163 - Rare. In oak forest openings.

\section{CAPRIFOLIACEAE}

*Lonicera alpina subsp. formanekiana (Halácsy) Hayek - Pcaesp, Bk - Chas. 1330 (2/6/2011, 22.22358 E, $41.1342 \mathrm{~N}, 1470 \mathrm{~m}$, mixed beech - thermophilous deciduous stand on scree), Chas. 1697 (13/9/2011, 22.24146 E, 41.1561 N, $1670 \mathrm{~m}$, rivulet above treeline) - Rare. Screes and rivulets at treeline, on calcareous substrates.

*Lonicera etrusca G. Santi - P lian, Me - Chas. 1213 (20/5/2011, 22.226 E, 41.11965 N, 1030 m), Chas. 1451 (22/6/2011, 22.2091 E, 41.1147 N, 900 m) - Sporadic. In thermophilous deciduous shrublands and forests.

*Sambucus ebulus L. - G rhiz/H scap, ME - Chas. 1575 (22/7/2011, 22.19298 E, $41.12067 \mathrm{~N}, 1140 \mathrm{~m}$, forest road in beech stand) - Common at roads in beech and oak forests. Rare in grassy openings of beech and oak stands.

*Sambucus nigra L. - P caesp, EA - Chas. 710 (2/6/2010, 22.19442 E, 41.12006 N, $1130 \mathrm{~m}$, forest road in beech stand) - Sporadic. Forest roads and streambanks in beech stands.

*Viburnum opulus L. - P caesp, ES - Chas. 973 (13/3/2011, 22.20892 E, 41.11686 N, $960 \mathrm{~m}$, thermophilous deciduous shrubland in a mixed thermophilous deciduous beech stand) - Rare.

\section{CARYOPHYLLACEAE}


Arenaria cretica Sprengel - C suffr, Bk - Chas. 1634 (18/8/2011, 22.2297 E, 41.14574 $\mathrm{N}, 1930 \mathrm{~m}$, subalpine sparse grassland on rocky ground), Chas. 2384 (20/7/2013, $22.23308 \mathrm{E}, 41.14444 \mathrm{~N}, 2030 \mathrm{~m}$, subalpine sparse grassland on rocky ground), MFG1: 88, FH1: map 258 - Rare. On calcareous, rocky and stony open ground above treeline.

*Arenaria leptoclados (Rchb.) Guss. - T scap, EA - Chas. 1077 (22/4/2011, 22.23431 $\mathrm{E}, 41.1205 \mathrm{~N}, 960 \mathrm{~m}$, grassy opening in thermophilous deciduous stand), Chas. 1111 (26/4/2011, 22.19856 E, 41.104 N, 640 m, open shrubland of Juniperus oxycedrus and thermophilous deciduous) - Common. Grasslands and phrygana on stony ground. Up to $1500 \mathrm{~m}$.

*Arenaria serpyllifolia L. - T scap, EA - Chas. 2045 (21/6/2012, 22.22206 E, 41.14625 N, 1760 m), Chas. 2099 (30/6/2012, 22.22507 E, 41.15404 N, 1840 m - Sporadic. In subalpine grasslands, mainly on stony ground.

Atocion armeria (L.) Raf. - H bienn/T scap, ES - Chas. 246 (28/8/2009, 22.21245 E, $41.12746 \mathrm{~N}, 1290 \mathrm{~m}$, forest road in beech stand), Chas. 858 (4/7/2010, 22.18525 E, $41.1373 \mathrm{~N}, 1370 \mathrm{~m}$, stony slope in open beech stand), FH1: map 562 (as Silene armeria L.), MFG1: 166 (as Silene armeria L.) - Sporadic. Siliceous stony, open ground, between 800 and $1800 \mathrm{~m}$.

Cerastium banaticum (Rochel) Heuff. subsp. banaticum - H caesp, BA - Chas. 252 (28/8/2009, 22.21319 E, 41.14404 N, 1760 m, subalpine shrubland), Chas. 619 (19/5/2010, 22.21624 E, $41.11932 \mathrm{~N}, 1110 \mathrm{~m}$, open shrubland of thermophilous deciduous and Juniperus oxycedrus), Chas. 794 (12/6/2010, 22.18793 E, 41.13977 $\mathrm{N}, 1400 \mathrm{~m}$, forest road in beech stand), Chas. 1194 (20/5/2011, 22.22885 E, $41.11564 \mathrm{~N}, 890 \mathrm{~m}$, phrygana on stony ground), MFG1: 115, FH1: map $385-$ Common. Grasslands, phrygana and open shrublands, between 700 and $2000 \mathrm{~m}$.

*Cerastium brachypetalum subsp. roeseri (Boiss. \& Heldr.) Nyman - T scap, Me Chas. $1082(22 / 4 / 2011,22.23431$ E, $41.1205 \mathrm{~N}, 960 \mathrm{~m}$, open shrubland of Juniperus oxycedrus and thermophilous deciduous), Chas. 1145 (1/5/2011, 22.22637 E, 41.11488 N, 840 m, phrygana), Chas. 1373 (9/6/2011, 22.19761 E, $41.15172 \mathrm{~N}, 1730 \mathrm{~m}$, grassy opening in subalpine shrubland) - Common. Grasslands, phrygana and open shrublands, between 600 and $1950 \mathrm{~m}$.

*Cerastium glomeratum Thuill. - T scap, Co - Chas. 1029 (8/4/2011, 22.19853 E, $41.10508 \mathrm{~N}, 680 \mathrm{~m}$, grassy opening in shrubland of Juniperus oxycedrus and thermophilous deciduous) - Sporadic. In grasslands and phrygana of lowland areas.

*Cerastium glutinosum Fr. - T scap, EA - Chas. 1028 (8/4/2011, 22.19853 E, 41.10508 $\mathrm{N}, 680 \mathrm{~m}$, grassy opening in shrubland of Juniperus oxycedrus and thermophilous deciduous), Chas. 1118 (26/4/2011, 22.19856 E, 41.104 N, 640 m, grassy opening in shrubland of Juniperus oxycedrus and thermophilous deciduous) - Common. In grasslands and phrygana of lowland areas.

*Cerastium holestoides subsp. vulgare (Fr.) Buttler - H scap, Eu - Chas. 1318 (2/6/2011, 22.21895 E, $41.14233 \mathrm{~N}, 1610 \mathrm{~m}$, swampy place at treeline), Chas. 1371 (9/6/2011, 22.1989 E, $41.15247 \mathrm{~N}, 1740 \mathrm{~m}$, stream above treeline), Chas. 2044 (21/6/2012, 22.22206 E, 41.14625 N, 1760 m, subalpine grassland) - Sporadic. Damp places at and above treeline and mesic subalpine grasslands.

Cerastium ramosissimum Boiss. - Tscap, Me - Voliotis 1983: 163 (as Cerastium gracile Dufour), FH1: map 413 (as Cerastium gracile Dufour).

Cerastium rectum subsp. petricola (Pančić) Gartner - Tscap, Bk - Chas. 475 (17/4/2010, 22.21942 E, 41.11621 N, 930 m, phrygana), Chas. 493 (25/4/2010, 
$22.19838 \mathrm{E}, 41.10616 \mathrm{~N}, 720 \mathrm{~m}$, grassy opening in shrubland of Juniperus oxycedrus and thermophilous deciduous), Chas. 1064 (16/4/2011, 22.24779 E, $41.13331 \mathrm{~N}, 1130 \mathrm{~m}$, Quercus frainetto stand), Chas. 1372 (9/6/2011, 22.19761 E, $41.15172 \mathrm{~N}, 1720 \mathrm{~m}$, grassy opening in subalpine shrubland), Chas. 2081 (25/6/2012, 22.20738 E, 41.14728 N, 2010 m, subalpine grassland), MFG1: 121 Common. Grasslands, phrygana and open thermophilous deciduous stands, between 600 and $2050 \mathrm{~m}$.

*Cerastium semidecandrum L. - T scap, EA - Chas. 1027 (8/4/2011, 22.23559 E, $41.11295 \mathrm{~N}, 700 \mathrm{~m}$, open shrubland of Juniperus oxycedrus and thermophilous deciduous), Chas. 1030 (8/4/2011, 22.22872 E, 41.11131 N, 660 m, dry grassland) - Common. Grasslands, phrygana and open shrublands of lowland areas.

Dianthus armeria L. - H bienn, ES - Chas. 2162 (6/7/2012, 22.288 E, 41.13563 N, 700 $\mathrm{m}$, forest road in Quercus frainetto stand), Parent 2005: 203 - Rare.

Dianthus corymbosus Sm. - C suffr, Gr - Chas. 1462 (27/6/2011, 22.20003 E, $41.11979 \mathrm{~N}, 1100 \mathrm{~m}$, forest road in mixed beech - thermophilous deciduous stand), Chas. $1481(27 / 6 / 2011,22.21511$ E, $41.12146 \mathrm{~N}, 1070 \mathrm{~m}$, open shrubland of Juniperus oxycedrus and thermophilous deciduous), Chas. 2260 (5/6/2013, $22.20666 \mathrm{E}, 41.12115 \mathrm{~N}, 1070 \mathrm{~m}$, forest road in mixed beech - thermophilous deciduous stand), Voliotis 1983: 163 - Sporadic. Forest roads, grasslands and open shrublands, between 800 and $1200 \mathrm{~m}$.

Dianthus deltoides L. subsp. deltoides - H caesp, ES - Chas. 917 (1/8/2010, 22.21575 E, $41.15939 \mathrm{~N}, 2030 \mathrm{~m}$, grassy opening in subalpine shrubland), Chas. 2075 $(21 / 6 / 2012,22.22243 \mathrm{E}, 41.1459 \mathrm{~N}, 1760 \mathrm{~m}$, grassy opening in subalpine shrubland), MFG1: 184, FH1: map 675 - Common. Mesic grasslands at and above treeline.

*Dianthus giganteus d'Urv. - C suffr, BA - Chas. 174 (13/8/2009, 22.18554 E, $41.12244 \mathrm{~N}, 950 \mathrm{~m}$, grassy opening in beech stand), Chas. 354 (15/9/2009, 22.26424 E, 41.16013 N, 1540 m, grassland at treeline) - Sporadic. Grasslands. 800 $-1700 \mathrm{~m}$.

Dianthus integer subsp. minutiflorus (Halácsy) Bornm. - H caesp, Bk - Chas. 918 (1/8/2010, 22.218 E, $41.16006 \mathrm{~N}, 1980 \mathrm{~m}$, subalpine shrubland), Chas. 1309 (2/6/2011, 22.22503 E, 41.14233 N, 1620 m, shrubland Juniperus communis subsp. nana at treeline), FH1: 347/ map 663 - Common. Grasslands and shrublands at and above treeline.

Dianthus pinifolius subsp. lilacinus (Boiss. \& Heldr.) Wettst. - H caesp, Bk - Chas. $860(4 / 7 / 2010,22.19325 \mathrm{E}, 41.11518 \mathrm{~N}, 910 \mathrm{~m}$, forest road in thermophilous deciduous stand), Chas. 2041 (18/6/2012, 22.23434 E, 41.13302 N, 1380 m, grassy opening in beech - thermophilous deciduous stand, on stony ground), FH1: map 712 - Sporadic. Grasslands and phrygana on stony ground. From $700 \mathrm{~m}$ up to the treeline.

*Dianthus stenopetalus Griseb. - H scap, Bk - Chas. 2425 (26/5/2014, 22.28776 E, 41.13572 N, 710 m, dry grassland within oak stand) - Rare.

Dianthus viscidus Bory \& Chaub. - H scap, BA - Chas. 1943 (30/5/2012, 22.19717 E, $41.111 \mathrm{~N}, 840 \mathrm{~m}$, open shrubland of thermophilous deciduous and Juniperus oxycedrus), Chas. 1978 (4/6/2012, 22.25307 E, 41.10934 N, 630 m, dry grassland), FH1: map 697 - Sporadic. Dry grasslands and open shrublands of lowland areas.

Heliosperma pusillum subsp. albanicum (K. Malý) Niketić \& Stevan - H caesp, Bk Chas. 270 (28/8/2009, 22.21712 E, 41.15284 N, 1860 m, streambank), Chas. 1568 
(22/7/2011, 22.17604 E, 41.15589 N, 1830 m, streambank), MFG1: 165 (as Silene pusilla subsp. albanica (K. Malý) Greuter \& Burdet), FH1: 294/ map 565 (as Silene pusilla subsp. albanica (K. Malý) Greuter \& Burdet) - Common. Streambanks, fens and springs above treeline.

*Herniaria glabra L. - T scap, Pt - Chas. 427 (31/10/2009, 22.21237 E, 41.13016 N, $1320 \mathrm{~m}$, forest road in beech stand), Chas. 1101 (26/4/2011, 22.20094 E, 41.10836 N, 770 m, open shrubland of thermophilous deciduous and Juniperus oxycedrus) Sporadic. Forest roads, dry grasslands and open shrublands of lowland areas.

*Herniaria incana Lam. - H caesp, EA - Chas. 1273 (28/5/2011, 22.19337 E, 41.11509 $\mathrm{N}, 910 \mathrm{~m}$, forest road in thermophilous deciduous stand), Chas. 2201 (27/7/2012, 22.25705 E, 41.1076 N, 610 m, dry grassland) - Sporadic. Dry grasslands and forest roads of lowland areas.

Lychnis coronaria L. (Desr.) - H scap, MS - Chas. 31 (11/7/2009, 22.18 E, 41.12893 N, $1000 \mathrm{~m}$, beech stand), FH1: map 470 (as Silene coronaria (L.) Clairv.) Sporadic. Oak stands and thermophilous beech forests.

*Lycnhis subintegra (Hayek) Turrill - H scap, Bk - Chas. 1315 (2/6/2011, 22.2249 E, 41.1426 N, 1620 m, streambank at treeline) - Rare.

* Minuartia attica (Boiss. \& Spruner) Vierh. subsp. attica - H caesp/C suffr, Bk - Chas. $474(17 / 4 / 2010,22.218 \mathrm{E}, 41.12025 \mathrm{~N}, 1140 \mathrm{~m}$, sparse grassland on rocky ground), Chas. $579(12 / 5 / 2010,22.24605 \mathrm{E}, 41.14049 \mathrm{~N}, 1480 \mathrm{~m}$, sparse grassland on stony ground), Chas. $735(2 / 6 / 2010,22.20122 \mathrm{E}, 41.13737 \mathrm{~N}, 1790 \mathrm{~m}$, subalpine grassland), Chas. 1032 (8/4/2011, 22.23725 E, 41.11307 N, 700 m, open shrubland of Juniperus oxycedrus and thermophilous deciduous), Chas. 2093 (25/6/2012, 22.20234 E, 41.14874 N, 1950 m, subalpine sparse grassland) - Common. All-over the mountain in sparse grasslands and phrygana, on stony ground.

Minuartia garckeana (Boiss.) Mattf. - H caesp, BA - Chas. 172 (13/8/2009, 22.18554 E, $41.12244 \mathrm{~N}, 950 \mathrm{~m}$, rocky opening in beech stand), Chas. 231 (24/8/2009, 22.21624 E, $41.12382 \mathrm{~N}, 1070 \mathrm{~m}$, open thermophilous deciduous shrubland on rocky ground), MFG1: 106, FH1: map 342 - Common. Rocky and stony places with sparse vegetation, between 600 and $1800 \mathrm{~m}$.

Minuartia glomerata subsp. velutina (Boiss. \& Orph.) Mattf. - H scap, Bk - Chas. 615 (19/5/2010, 22.21658 E, 41.11554 N, 920 m, phrygana), Chas. 891 (24/7/2010, $22.2568 \mathrm{E}, 41.14849 \mathrm{~N}, 1570 \mathrm{~m}$, grassy opening in beech stand), Voliotis 1983: 163, FH1: map 327 - Common. Rocky and stony calcareous places with sparse vegetation, between 600 and $1700 \mathrm{~m}$.

* Minuartia mesogitana (Boiss.) Hand.-Mazz. cf. - T scap, BA - Chas. 1031 (8/4/2011, 22.23725 E, 41.11307 N, $700 \mathrm{~m}$, phrygana), Chas. 1050 (11/4/2011, $22.22454 \mathrm{E}$, $41.11179 \mathrm{~N}, 680 \mathrm{~m}$, phrygana), Chas. 1069 (22/4/2011, 22.24038 E, 41.11636 N, $720 \mathrm{~m}$, open shrubland of Juniperus oxycedrus and thermophilous deciduous) Common. Open shrublands, phrygana and dry grasslands of lowland areas.

* Minuartia recurva subsp. condensata (C. Presl) Greuter \& Burdet - H caesp/C pulv, ME - Chas. 1630 (18/8/2011, 22.22777 E, 41.1467 N, 1890 m, subalpine sparse grassland), Chas. 2068 (21/6/2012, $22.22265 \mathrm{E}, 41.14653 \mathrm{~N}, 1780 \mathrm{~m}$, grassy opening in subalpine shrubland), Chas. 2315 (22/6/2013, 22.19957 E, $41.15842 \mathrm{~N}$, $1900 \mathrm{~m}$, subalpine sparse grassland) - Sporadic. Stony and rocky ground with sparse vegetation above treeline.

Minuartia stojanovii (Kitanov) Kožuharov \& Kuzmanov - H caesp/C pulv, Bk - Chas. 1633 (18/8/2011, 22.23296 E, 41.14435 N, 2030 m, subalpine sparse grassland), 
Chas. $2387(20 / 7 / 2013,22.23308$ E, 41.14426 N, $2030 \mathrm{~m}$, subalpine sparse grassland), MFG1: 94 (as M. setaceae (Thuill.) Hayek), FH1: 180/ map 336, Schuler 2004: tab. 21 - Rare. Stony and rocky ground with sparse vegetation above treeline.

Minuartia verna subsp. collina (Neilr.) Domin - H caesp, ME - Chas. 640 (19/5/2010, 22.21624 E, 41.11932 N, $1120 \mathrm{~m}$, open shrubland of Juniperus oxycedrus and thermophilous deciduous), Chas. 1210 (20/5/2011, $22.22886 \mathrm{E}, 41.11501 \mathrm{~N}, 850 \mathrm{~m}$, phrygana), Chas. 1240 (25/5/2011, 22.2208 E, $41.10921 \mathrm{~N}, 640$ m, dry grassland), FH1: map 348 - Sporadic. Phrygana and dry grasslands up to the treeline.

*Moehringia trinervia (L.) Clairv. - T scap, ES - Chas. 1156 (6/5/2011, 22.26833 E, 41.14975 N, 1260 m), Chas. 1484 (27/6/2011, 22.21597 E, 41.12125 N, 1070 m) Common. Mesic beech stands.

*Moenchia graeca Boiss. \& Heldr. - T scap, BA - Chas. 1107 (26/4/2011, 22.19856 E, $41.104 \mathrm{~N}, 640 \mathrm{~m}$, grassy opening in shrubland of Juniperus oxycedrus and thermophilous deciduous) - Sporadic. Phrygana and dry grasslands of lowland areas.

*Moenchia mantica (L.) Bartl. - T scap, ME - Chas. 1095 (26/4/2011, 22.20079 E, $41.10935 \mathrm{~N}, 810 \mathrm{~m}$, dry grassland) - Sporadic. Forest roads, grasslands and forest openings at lowland areas.

Paronychia albanica subsp. graeca Chaudhri - H caesp, Gr - Chas. 675 (24/5/2010, 22.24558 E, $41.15805 \mathrm{~N}, 1790 \mathrm{~m}$, subalpine sparse grassland on stony ground), Chas. $1146(1 / 5 / 2011,22.22722 \mathrm{E}, 41.11426 \mathrm{~N}, 820 \mathrm{~m}$, phrygana on rocky ground), Chas. $1632(18 / 8 / 2011,22.23295$ E, $41.14431 \mathrm{~N}, 2030 \mathrm{~m}$, subalpine sparse grassland on rocky ground), FH1: 225/ map 438 - Common. All-over the mountain, on rocky and stony calcareous ground with sparse vegetation.

? Paronychia chionaea Boiss. - H caesp, BA - Voliotis 1983: 163 (as P. kapela subsp. chionaea (Boiss.) Borhidi).

Paronychia macedonica Chaudhri - H caesp, Bk - MFG: 128, Schuler 2004: tab. 21.

*Petrorhagia dubia(Raf.) G. López\&Romo - Tscap, Me - Chas. 715 (2/6/2010, $22.19336 \mathrm{E}, 41.11536 \mathrm{~N}, 910 \mathrm{~m}$, forest road in thermophilous deciduous stand), Chas. 1268 (28/5/2011, 22.20473 E, 41.10896 N, 770 m, dry grassland) - Sporadic. Forest roads and grasslands of lowland areas.

*Petrorhagia illyrica (Ard.) P. W. Ball \& Heywood subsp. illyrica - H caesp, Bk Chas. $892(24 / 7 / 2010,22.25541 \mathrm{E}, 41.14712 \mathrm{~N}, 1530 \mathrm{~m}$, stony open place in beech stand), Chas. 1520 (7/7/2011, 22.22498 E, 41.11342 N, 720 m, phrygana on stony ground) - Sporadic. Stony and rocky places with sparse vegetation. $600-1700 \mathrm{~m}$.

*Petrorhagia prolifera (L.) P. W. Ball \& Heywood - T scap, EA - Chas. 1361 (9/6/2011, $22.2074 \mathrm{E}, 41.11152 \mathrm{~N}, 820 \mathrm{~m}$, forest road in thermophilous deciduous stand), Chas. 1422 (16/6/2011, 22.22649 E, 41.11452 N, $830 \mathrm{~m}$, phrygana) Sporadic. Forest roads, phrygana and grasslands of lowland areas.

*Petrorhagia thessala (Boiss.) P. W. Ball \& Heywood - H caesp, Bk - Chas. 191 $(24 / 8 / 2009,22.21624 \mathrm{E}, 41.12382 \mathrm{~N}, 1070 \mathrm{~m}$, open thermophilous deciduous shrubland on rocky ground), Chas. 890 (24/7/2010, $22.22499 \mathrm{E}, 41.13504 \mathrm{~N}, 1560$ $\mathrm{m}$, stony open place at treeline), Chas. 931 (8/8/2010, 22.22126 E, $41.12318 \mathrm{~N}$, $1000 \mathrm{~m}$, open thermophilous deciduous shrubland) - Sporadic. Stony and rocky calcareous places with sparse vegetation, between 600 and $1600 \mathrm{~m}$.

*Sagina procumbens L. - H caesp, Ct - Chas. 1531 (12/7/2011, 350260 E, 4556270 N, $1790 \mathrm{~m}$, spring above treeline) - Rare. 
Saponaria bellidifolia Sm. - H caesp, Me - Chas. 1532 (12/7/2011, 22.21741 E, $41.14654 \mathrm{~N}, 1850 \mathrm{~m}$, subalpine sparse grassland on stony ground), Chas. 2033 (18/6/2012, $22.22939 \mathrm{E}, 41.15312 \mathrm{~N}, 1360 \mathrm{~m}$, sparse grassland on stony ground), MFG1: 173, FH1: map 633 - Sporadic. Calcareous stony places with sparse vegetation, between 1350 and $2000 \mathrm{~m}$.

*Saponaria glutinosa M. Bieb. - T scap/H bienn, ME - Chas. 2259 (5/6/2013, 22.23935 E, 41.13255 N, $1200 \mathrm{~m}$, thermophilous deciduous stand within beech stand) - Rare.

*Saponaria officinalis L. - H scap, ES - Chas. 342 (15/9/2009, 22.27384 E, 41.15307 $\mathrm{N}, 1150 \mathrm{~m}$, forest road in mixed beech - oak stand) - Sporadic. Forest roads and streambanks in grasslands of lowland areas.

Scleranthus perennis subsp. marginatus (Guss.) Nyman - H caesp, Me - Chas. 544 (8/5/2010, 22.22281 E, 41.14959 N, 1750 m), Chas. 701 (2/6/2010, 22.20122 E, $41.13737 \mathrm{~N}, 1790 \mathrm{~m})$, MFG1: 125, FH1: map 424 - Sporadic. In subalpine grasslands on stony ground.

Silene bupleuroides subsp. staticifolia (Sm.) Chowdhuri - H caesp, BA - Chas. 2355 $(1 / 7 / 2013,22.22534 \mathrm{E}, 41.13522 \mathrm{~N}, 1590 \mathrm{~m}$, rocky open slope at treeline), MFG1: 142, FH1: map 507 - Sporadic. Rocky and stony calcareous places with sparse vegetation at and above treeline.

Silene ciliata subsp. graefferi (Guss.) Nyman - H caesp, BI - Chas. 1625 (18/8/2011, $22.23031 \mathrm{E}, 41.14548 \mathrm{~N}, 1950 \mathrm{~m}$, subalpine sparse grassland on stony ground), MFG1: 164, FH1: map 599 - Sporadic. Rocky and stony calcareous places with sparse vegetation at and above treeline.

*Silene conica L. - T scap, EA - Chas. 507 (2/5/2010, 22.22395 E, 41.11187 N, 680 m, dry grassland), Chas. 1070 (22/4/2011, 22.23766 E, 41.11136 N, 680 m, phrygana) - Common. Dry grasslands and phrygana of lowland areas.

Silene damboldtiana Greuter \& Melzh. - H caesp, Bk - Chas. 2280 (14/6/2013, 22.2687 E, $41.16272 \mathrm{~N}, 1560 \mathrm{~m}$, stony place in grassland at treeline), Chas. 2300 (18/6/2013, 22.23405 E, 41.13671 N, 1590 m, grassland at treeline), Melzheimer \& Greuter 1979: 613 - Common. Grasslands on stony calcareous ground at and above treeline. Between 1200 and $1900 \mathrm{~m}$.

*Silene fabarioides Hausskn. - H bienn/H scap, Bk - Chas. 853 (26/6/2010, 22.22397 E, $41.12853 \mathrm{~N}, 1440 \mathrm{~m}$, open thermophilous deciduous stand on rocky slope) Rare.

*Silene gallinyi Rchb. - T scap, BI - Chas. 2163 (6/7/2012, 22.288 E, 41.13563 N, 700 $\mathrm{m}$, forest road in Quercus frainetto stand) - Rare.

Silene gigantea subsp. rhodopea (Janka) Greuter - H scap, BA - Chas. 1406 (16/6/2011, 22.22684 E, 41.11507 N, 860 m, phrygana), FH1: map 492 - Sporadic. Open shrublands, phrygana and dry grasslands at low altitudes.

Silene italica (L.) Pers. Subsp. italica - H ros, - Chas. 1080 (22/4/2011, 22.23481 E, $41.1196 \mathrm{~N}, 890 \mathrm{~m}$, thermophilous deciduous stand), Chas. 1828 (5/5/2012, $22.19804 \mathrm{E}, 41.11849 \mathrm{~N}, 1080 \mathrm{~m}$, forest road in mixed beech - thermophilous deciduous stand), Voliotis 1983: 163, FH1: map 482 - Common. Forests and shrublands from $600 \mathrm{~m}$ up to the lower and drier parts of beech forest zone.

Silene latifolia Poir. - H bienn, EA - Chas. 39 (11/7/2009, 22.18321 E, 41.12484 N, $980 \mathrm{~m}$, grassy opening in beech stand), Chas. $780(12 / 6 / 2010,22.18915 \mathrm{E}$, $41.13439 \mathrm{~N}, 1370 \mathrm{~m}$, forest road in beech stand), Chas. $2022(13 / 6 / 2012,22.22851$ 
E, $41.12365 \mathrm{~N}, 1280 \mathrm{~m}$, grassy clearing in thermophilous deciduous stand), FH1: map 474 - Sporadic. In various habitats of lowland areas, mainly by forest roads.

*Silene otites (L.) Wibel - H ros, ES - Chas. 1274 (28/5/2011, 22.22133 E, 41.11156 N, $690 \mathrm{~m}$, forest road in shrubland of Juniperus oxycedrus and thermophilous deciduous), Chas. 1893 (24/5/2012, 22.20121 E, $41.11161 \mathrm{~N}, 870 \mathrm{~m}$, open shrubland of Juniperus oxycedrus and thermophilous deciduous) - Sporadic. Open shrublands and phrygana of lowland areas.

*Silene paradoxa L. - H ros, Me - Chas. 859 (4/7/2010, 22.18458 E, 41.13585 N, 1280 $\mathrm{m}$, open shrubland of Juniperus oxycedrus and thermophilous deciduous), Chas. $2118(30 / 6 / 2012,22.1931 \mathrm{E}, 41.11625 \mathrm{~N}, 950 \mathrm{~m}$, forest road in mixed thermophilous deciduous - beech stand) - Sporadic. Open shrublands and phrygana on stony ground and forest roads, at low altitudes.

*Silene parnassica Boiss. \& Spruner - H caesp, Bk - Chas. 2090 (25/6/2012, 22.21267 E, $41.15007 \mathrm{~N}, 1990 \mathrm{~m}$, streambank above treeline) - Rare.

Silene radicosa Boiss. \& Heldr. - H ros, Bk - Chas. 883 (24/7/2010, 22.25749 E, $41.14949 \mathrm{~N}, 1570 \mathrm{~m}$, stony place in sparse grassland within beech stand), Chas. 957 (14/8/2010, 22.22499 E, 41.11279 N, 690 m), MFG1: 143, FH1: map 497 Common. Open shrublands, phrygana and sparse grasslands on stony calcareous ground. Between 600 and $2050 \mathrm{~m}$.

Silene roemeri subsp. macrocarpa (Vandas) Greuter - H ros, Bk - Chas. 909 (1/8/2010, 22.21225 E, 41.16096 N, $2120 \mathrm{~m}$, subalpine grassland), Chas. 2064 (21/6/2012, 22.22315 E, $41.14591 \mathrm{~N}, 1780 \mathrm{~m}$, grassy opening in subalpine shrubland), MFG1: 145 (as $S$. roemeri Friv.), FH1: map 512 - Common. In subalpine mesic grasslands.

Silene saxifraga L. - H caesp, ME - Chas. 1629 (18/8/2011, 22.2297 E, 41.14574 N, $1930 \mathrm{~m}$, subalpine sparse grassland on stony slope), Chas. $2187(15 / 7 / 2012$, 22.24233 E, $41.15476 \mathrm{~N}, 1730 \mathrm{~m}$, sparse grassland on stony ground), MFG1: 154, FH1: map 543 - Sporadic. Calcareous screes and stony or rocky calcareous ground with sparse vegetation, above treeline.

Silene subconica Friv. - T scap, Me - Chas. 1984 (4/6/2012, 22.24855 E, 41.1181 N, $760 \mathrm{~m}$, open shrubland of Juniperus oxycedrus and thermophilous deciduous), Chas. $2019(13 / 6 / 2012,22.22861$ E, $41.11998 \mathrm{~N}, 1110 \mathrm{~m}$, grassy clearing in thermophilous deciduous stand), Voliotis 1983: 163 (as S. conica subsp. subconica (Friv.) Gavioli) - Sporadic. Dry grasslands, phrygana and open shrublands, at low altitudes.

Silene supina M. Bieb. - H caesp/C suffr, BA - Chas. 1548 (17/7/2011, 22.22049 E, $41.11191 \mathrm{~N}, 690 \mathrm{~m}$, forest road in Juniperus oxycedrus - thermophilous deciduous shrubland), MFG1: 153, FH1: 269/ map 517 - Rare. Open shrublands and phrygana on stony ground, at lowland areas.

Silene viridiflora L. - H ros, ES - Chas. 2156 (6/7/2012, 22.25119 E, 41.12634 N, 850 m, Quercus pubescens stand), FH1: map 477 - Sporadic. Thermophilous beech forests and oak forests.

Silene vulgaris (Moench) Garcke - H scap, Co - Chas. 40 (11/7/2009, 22.18 E, $41.12893 \mathrm{~N}, 1000 \mathrm{~m}$, beech stand), Chas. 1602 (13/8/2011,22.21372 E, 41.15963 N, 2090 m, subalpine shrubland), Chas. 1864 (16/5/2012, 22.19804 E, 41.1184 N, $1080 \mathrm{~m}$, forest road in mixed beech and thermophilous deciduous stand), FH1: map 527 - Sporadic. In beech and oak forests and subalpine shrublands and grasslands.

*Spergularia rubra (L.) J. Presl \& C. Presl - T scap/C suffr, Pt - Chas. 2177 $(11 / 7 / 2012,22.18042 \mathrm{E}, 41.1311 \mathrm{~N}, 1050 \mathrm{~m}$, damp place by roadside in beech 
stand), Chas. 2328 (26/6/2013, 22.22155 E, 41.14768 N, 1790 m, roadside above treeline) - Rare.

*Stellaria alsine Grimm - H scap, ES - Chas. 2330 (26/6/2013, 22.23237 E, 41.16181 $\mathrm{N}, 1780 \mathrm{~m}$, spring above treeline) - Rare.

*Stellaria aquatica (L.) Scop. - H scap, ES - Chas. 343 (15/9/2009, 22.27132 E, $41.15376 \mathrm{~N}, 1170 \mathrm{~m}$, forest road in beech stand) - Rare.

Stellaria graminea L. - H scap, ES - Chas. 333 (15/9/2009, 22.25928 E, 41.16285 N, 1750 m), Chas. 1546 (12/7/2011, 22.2308 E, 41.15359 N, 1860 m), FH1: map 375 - Common. Mesic subalpine grasslands.

*Stellaria media (L.) Cirillo - T rept/H bienn, Co - Chas. 1033 (8/4/2011, 22.20991 E, $41.11111 \mathrm{~N}, 790 \mathrm{~m}$, grassy clearing in thermophilous deciduous stand), Chas. 1374 (9/6/2011, 22.19656 E, $41.15063 \mathrm{~N}, 1710 \mathrm{~m}$, grassy opening in subalpine shrubland) - Sporadic. Grasslands and roadsides. Between 600 and $1800 \mathrm{~m}$.

*Stellaria montana Pierrat - H scap, Eu - Chas. 1375 (9/6/2011, 22.18866 E, 41.15221 N, $1580 \mathrm{~m}$, beech stand at treeline), Chas. 2132 (30/6/2012, 22.22101 E, 41.15884 $\mathrm{N}, 1860 \mathrm{~m}$, above treeline tall-herb vegetation) - Sporadic. Mesic beech stands at treeline and tall-herb vegetation above treeline.

Viscaria atropurpurea Griseb. - H scap, Bk - Chas. 499 (25/4/2010, 22.19058 E, $41.11261 \mathrm{~N}, 720 \mathrm{~m}$, grassland within thermophilous deciduous stand), Chas. 667 (24/5/2010, 22.26519 E, $41.15564 \mathrm{~N}, 1280 \mathrm{~m}$, beech stand), Voliotis 1983: 163 (Lychnis viscaria subsp. atropurpurea (Griseb.) Chater), FH1: map 473 (as Silene atropurpurea (Griseb.) Greuter \& Burdet) - Common. Beech stands, forest roads in oak and beech forests and grasslands. Between 700 and $1700 \mathrm{~m}$.

\section{CELASTRACEAE}

*Euonymus europaeus L. - P caesp/P scap, EA - Chas. 1734 (19/10/2011, 22.21895 E, $41.10729 \mathrm{~N}, 620 \mathrm{~m}$, thermophilous deciduous stand in dry grassland) - Rare.

*Euonymus latifolius (L.) Mill. - P caesp/P scap, EA - Chas. 661 (24/5/2010, 22.25993 E, $41.15629 \mathrm{~N}, 1400 \mathrm{~m})$, Chas. $1510(2 / 7 / 2011,22.27601 \mathrm{E}, 41.14862 \mathrm{~N}, 1030 \mathrm{~m})$, Chas. 1683 (10/9/2011, 22.26263 E, 41.15786 N, 1420 m) - Common. In beech forests, mainly on stony ground.

*Euonymus verrucosus Scop. - P caesp, Eu - Chas. 412 (17/10/2009, 22.24349 E, $41.13361 \mathrm{~N}, 1250 \mathrm{~m}$, open thermophilous deciduous stand), Chas. 625 (19/5/2010, $22.2155 \mathrm{E}, 41.11597 \mathrm{~N}, 980 \mathrm{~m}$, thermophilous deciduous stand) - Common in thermophilous deciduous shrublands and forests. Sporadic in oak and thermophilous beech forests.

\section{CHENOPODIACEAE}

*Atriplex patula L. - T scap, ES - Chas. 1678 (10/9/2011, 22.26648 E, 41.15179 N, $1230 \mathrm{~m}$, roadside in beech stand) - Rare.

*Blitum bonus-henricus (L.) Rchb. - H scap, - Chas. 350 (15/9/2009, 22.26424 E, $41.16013 \mathrm{~N}, 1530 \mathrm{~m}$, Pteridium thicket at treeline), Chas. 1857 (16/5/2012, $22.21739 \mathrm{E}, 41.14293 \mathrm{~N}, 1640 \mathrm{~m}$, tall-herb vegetation at treeline) - Sporadic. Grassy gentle slopes at treeline and above, where live-stock is concentrated.

Blitum virgatum L. - T scap, EA- FH1: map 211 (as Chenopodium foliosum Asch.).

*Chenopodium album L. - T scap, Co - Chas. 1676 (10/9/2011, 22.26224 E, 41.14092 $\mathrm{N}, 1120 \mathrm{~m}$, forest road in mixed oak - beech stand) - Common. Forest roads up to $1200 \mathrm{~m}$. 
Chenopodium vulvaria L. - T scap, EA - FH1: map 219.

Dysphania botrys (L.) Mosyakin \& Clemants - T scap, EA - Chas. 390 (27/9/2009, $22.21237 \mathrm{E}, 41.13016 \mathrm{~N}, 1320 \mathrm{~m}$, forest road in beech stand), FH1: map 226 (as Chenopodium botrys) - Rare.

\section{CISTACEAE}

Cistus creticus subsp. eriocephalus (Viv.) Greuter \& Burdet - C frut, Me- Chas. 292 $(10 / 9 / 2009,22.26411 \mathrm{E}, 41.1287 \mathrm{~N}, 780 \mathrm{~m}$, opening in Quercus frainetto stand), Voliotis 1983: 163 (as C. incanus L. subsp. incanus) - Common. In formations of lowland areas.

*Fumana scoparia Pomel - C suffr, Me - Chas. 433 (14/11/2009, 22.22415 E, $41.11295 \mathrm{~N}, 720 \mathrm{~m}$, phrygana), Chas. 840 (26/6/2010, $22.21917 \mathrm{E}, 41.1254 \mathrm{~N}$, $1220 \mathrm{~m}$, open shrubland of Juniperus oxycedrus and thermophilous deciduous), Chas. 1911 (24/5/2012, 22.22723 E, 41.11336 N, $750 \mathrm{~m}$, phrygana), Chas. 2350 (1/7/2013, $22.2279 \mathrm{E}, 41.13319 \mathrm{~N}, 1650 \mathrm{~m}$, sparse grassland on rocky slope) Common. At calcareous, stony or rocky places with sparse vegetation. $600-1700$ $\mathrm{m}$.

Fumana thymifolia (L.) Webb - C suffr, Me - Voliotis 1983: 163.

Helianthemum nummularium subsp. glabrum (W.D.J. Koch) Wilczek - C, Me - Parent 2005: 210 (as Helianthemum nitidum).

Helianthemum nummularium (L.) Miller subsp. nummularium - C suffr, Me - Chas. $339(15 / 9 / 2009,22.25927$ E, 41.16312 N, 1760 m), Chas. 382 (27/9/2009, 22.23223 E, $41.14505 \mathrm{~N}, 2000 \mathrm{~m})$, Chas. 593 (12/5/2010, 22.24445 E, 41.14227 N, $1580 \mathrm{~m}$ ), Voliotis 1983: 163 - Common. At stony and rocky places with sparse vegetation, between 600 and $2100 \mathrm{~m}$.

*Helianthemum nummularium subsp. tomentosum (Scop.) Schinz\&Thell. - C suffr, Me - Chas. 315 (10/9/2009, 22.25283 E, 41.13654 N, 1200 m) - Sporadic. On stony and rocky places with sparse vegetation. Up to $1400 \mathrm{~m}$.

Helianthemum oelandicum subsp. alpestre (Jacq.) Breistr. - C suffr, ME - Chas. 755 $(12 / 6 / 2010,22.18097 \mathrm{E}, 41.15858 \mathrm{~N}, 1820 \mathrm{~m}$, subalpine sparse grassland on stony ground), MFG1: 642 (as H. alpestre (Jacq.) DC.) - Sporadic. On stony and rocky places with sparse vegetation above treeline.

Helianthemum oelandicum subsp. canum (L.) Bonnier - C suffr, ME - Chas. 592 $(12 / 5 / 2010,22.24445 \mathrm{E}, 41.14227 \mathrm{~N}, 1580 \mathrm{~m}$, sparse grassland on stony slope at treeline), Chas. 655 (24/5/2010, $22.24793 \mathrm{E}, 41.15502 \mathrm{~N}, 1620 \mathrm{~m}$, sparse grassland on stony slope at treeline), Chas. $1021(8 / 4 / 2011,22.23911 \mathrm{E}, 41.1103 \mathrm{~N}, 660 \mathrm{~m}$, phrygana), Voliotis 1983: 163 (as H. canum (L.) Baumg. subsp. canum), MFG1: 642 (as H. canum (L.) Baumg. subsp. canum), Schuler 2004: tab. 21 - Common in stony slopes with sparse vegetation at and above treeline. Sporadic in phrygana and dry grasslands of lowland areas.

*Helianthemum salicifolium (L.) Miller - T scap, EA - Chas. 1020 (8/4/2011, 22.23725 E, 41.11307 N, 700 m, phrygana), Chas. 1108 (26/4/2011, 22.19856 E, $41.104 \mathrm{~N}, 640 \mathrm{~m}$, grassy opening in shrubland of Juniperus oxycedrus and thermophilous deciduous), Chas. 1275 (28/5/2011, 22.21002 E, 41.11156 N, $800 \mathrm{~m}$, grassy clearing in thermophilous deciduous and Juniperus oxycedrus stand) Common. Phrygana and dry grasslands. Up to $900 \mathrm{~m}$. 
*Tuberaria guttata (L.) Fourr. - T scap, MA - Chas. 1269 (28/5/2011, 22.20433 E, 41.10634 N, 700 m, dry grassland), Chas. 1351 (9/6/2011, 22.22068 E, $41.1093 \mathrm{~N}$, $640 \mathrm{~m}$, dry grassland) - Common. In phrygana and dry grasslands. Up to $900 \mathrm{~m}$.

\section{CONVOLVULACEAE}

*Calystegia sylvatica (Kit.) Griseb. - H scand, MS - Chas. 84 (27/7/2009, 22.1844 E, $41.1335 \mathrm{~N}, 1190 \mathrm{~m}$ ) - Sporadic. In mesic beech forests, at the lower part of their distribution.

*Convolvulus arvensis L. - G rhiz/H scap, Co - Chas. 1441 (22/6/2011, 22.2222 E, $41.11022 \mathrm{~N}, 650 \mathrm{~m}$, forest road in dry grassland) - Rare.

Convolvulus cantabrica L. - H scap, MS - Chas. 824 (26/6/2010, 22.22452 E, 41.11269 N, 700 m, dry grassland), Voliotis 1983: 164 - Sporadic. At forest roads, dry grasslands, phrygana and open shrublands, of lowland areas.

Cuscuta epithymum (L.) L. subsp. epithymum - T, EA - Parent 2005: 214.

*Cuscuta epithymum subsp. kotschyi (Desmoulins) Arcangeli - T par, ME - Chas. 264 (28/8/2009, 22.22506 E, 41.15458 N, 1840 m), Chas. 1607 (13/8/2011, 22.21497 E, $41.15749 \mathrm{~N}, 2060 \mathrm{~m})-$ Common. In grasslands at treeline and above.

*Cuscuta europaea L. - T par, Pt - Chas. 2176 (11/7/2012, 22.17897 E, 41.13198 N, $1060 \mathrm{~m}$, forest road in beech stand) - Rare.

\section{CORNACEAE}

Cornus mas L. - P caesp/P scap, EA - Chas. 370 (20/9/2009, 22.19392 E, 41.10816 N, $680 \mathrm{~m}$, thermophilous deciduous stand), Chas. $624(19 / 5 / 2010,22.2155 \mathrm{E}$, $41.11597 \mathrm{~N}, 980 \mathrm{~m}$, thermophilous deciduous stand), Voliotis 1983: 164 Common. In thermophilous deciduous forests and shrublands and in oak forests.

*Cornus sanguinea subsp. australis (C. A. Meyer) Jáv. - P caesp, EA - Chas. 900 $(1 / 8 / 2010,22.20692 \mathrm{E}, 41.1117 \mathrm{~N}, 830 \mathrm{~m}$, opening in thermophilous deciduous stand) - Rare. In stands and openings of thermophilous deciduous.

?Cornus sanguinea L. subsp. sanguinea - P caesp, ES - Voliotis 1983: 164.

\section{CRASSULACEAE}

*Hylotelephium telephium (L.) H. Ohba - H scap, ES - Chas. 288 (10/9/2009, $22.25007 \mathrm{E}, 41.14596 \mathrm{~N}, 1480 \mathrm{~m}$, rock in beech stand), Chas. 328 (15/9/2009, 22.26636 E, 41.16089 N, 1540 m, grassland on stony ground) - Sporadic. At stony and rocky places, mainly in beech stands.

Sedum acre L. - C succ, ES - Voliotis 1983: 164, FH2: map 1265.

*Sedum album L. - C succ, EA - Chas. 819 (26/6/2010, 22.22452 E, 41.11269 N, 700 $\mathrm{m}$, sparse grassland on stony ground), Chas. 1571 (22/7/2011, 22.17415 E, $41.15514 \mathrm{~N}, 1900 \mathrm{~m}$, sparse grassland on rocky streambank above treeline) Common. At screes and sparse grasslands on stony ground. From low to high altitudes.

*Sedum amplexicaule subsp. tenuifolium (Sm.) Greuter - C succ/H scap, Me - Chas. $1435(22 / 6 / 2011,22.20549 \mathrm{E}, 41.11185 \mathrm{~N}, 840 \mathrm{~m}$, stony ground of path in thermophilous deciduous stand) - Sporadic. Stony open places of lowland areas.

*Sedum annuum L. - T scap/H bienn, AA - Chas. 869 (4/7/2010, 22.18525 E, 41.1373 $\mathrm{N}, 1380 \mathrm{~m}$, sparse grassland on stony ground within beech stand), Chas. 2336 (26/6/2013, 22.19959 E, $41.14014 \mathrm{~N}, 1880 \mathrm{~m}$, subalpine sparse grassland on stony 
ground) - Sporadic. At screes and stony places with sparse vegetation at midaltitudes and above treeline.

Sedum atratum L. - T scap, Eu - Chas. 2464 (28/7/2014, 22.20978 E, 41.15534 N, $2080 \mathrm{~m}$, rocky silicate ground above treeline), MFG1: 356 - Rare.

Sedum cepaea L. - T scap, Me - Chas. 856 (4/7/2010, 22.18001 E, 41.12884 N, 1000 m, beech stand), Chas. 2106 (30/6/2012, 22.20444 E, $41.12391 \mathrm{~N}, 1210 \mathrm{~m}$, forest road in beech stand), FH2: map 1287 - Common. In oak and beech forests of lowland areas.

*Sedum dasyphyllum L. - C succ, Me - Chas. 2006 (13/6/2012, 22.22629 E, 41.11758 $\mathrm{N}, 940 \mathrm{~m}$, stony place in open thermophilous deciduous stand with Juniperus oxycedrus), Chas. 2453 (10/7/2014, 22.18557 E, 41.13865 N, 1450 m, rocky open place in beech forest) - Rare.

*Sedum grisebachii Boiss. \& Heldr. - H scap, BA - Chas. 2053 (21/6/2012, 22.21223 E, $41.1397 \mathrm{~N}, 1610 \mathrm{~m}$, rocky roadside in beech stand), Chas. $2063(21 / 6 / 2012$, 22.22264 E, $41.14266 \mathrm{~N}, 1650 \mathrm{~m}$, subalpine grassland) - Rare.

*Sedum hispanicum L. - H scap/C succ, EA - Chas. 873 (4/7/2010, 22.18525 E, $41.1373 \mathrm{~N}, 1380 \mathrm{~m}$, rocky opening in beech stand), Chas. 1938 (30/5/2012, $22.19326 \mathrm{E}, 41.11464 \mathrm{~N}, 880 \mathrm{~m}$, rocky opening in thermophilous deciduous stand), Chas. $2450(6 / 7 / 2014,22.19368$ E, 41.12995 N, 1430 m, open beech stand on stony ground) - Sporadic. At stony open places in the forest zone.

Sedum ochroleucum Chaix - C succ, Me - Chas. 822 (26/6/2010, 22.22452 E, $41.11269 \mathrm{~N}, 700 \mathrm{~m}$, phrygana), Chas. 2005 (8/6/2012, 22.22135 E, $41.11552 \mathrm{~N}$, $870 \mathrm{~m}$, phrygana on rocky ground), MFG1: 344, FH2: map 1284 - Common. At screes and other stony ground with sparse vegetation, between 700 and $2000 \mathrm{~m}$.

*Sedum rubens L. - T scap, MA - Chas. 2428 (14/6/2014, 22.20086 E, 41.11133 N, 860 m, phrygana) - Rare.

*Sedum urvillei DC. - C succ, ME - Chas. 331 (15/9/2009, 22.26009 E, 41.16376 N, $1800 \mathrm{~m}$, sparse grassland on stony ground), Chas. $771(12 / 6 / 2010,22.21621 \mathrm{E}$, 41.11166 N, 730 m, phrygana with Juniperus oxycedrus), Chas. 1465 (27/6/2011, $22.20086 \mathrm{E}, 41.11124 \mathrm{~N}, 850 \mathrm{~m}$, roadside in thermophilous deciduous stand) Common. On stony places with sparse vegetation. From low to high altitudes.

Sempervivum heuffelii Schott - C succ, Bk - Chas. 346 (15/9/2009, 22.26322 E, $41.16273 \mathrm{~N}, 1710 \mathrm{~m}), \mathrm{MFG} 1: 340, \mathrm{FH} 2$ : map 1263 - Common. At stony and rocky places with sparse vegetation, between 1100 and $2000 \mathrm{~m}$.

Umbilicus luteus (Huds.) Webb \& Berthel. - G bulb, Me - Chas. 866 (4/7/2010, 22.18641 E, $41.13849 \mathrm{~N}, 1400 \mathrm{~m}$ ), Voliotis 1983: 164 (as U. erectus DC.), FH2: map 1253 - Sporadic. At rocky places in beech stands.

*Umbilicus rupestris (Salisb.) Dandy - G bulb, MA - Chas. 1952 (30/5/2012, 22.19362 E, 41.11032 N, 750 m, thermophilous deciduous stand) - Rare.

\section{DIPSACACEAE}

*Cephalaria flava (Sm.) Szabó - H scap, Bk - Chas. 940 (8/8/2010, 22.22337 E, $41.12456 \mathrm{~N}, 1150 \mathrm{~m})$, Chas. 1710 (24/9/2011, $22.23719 \mathrm{E}, 41.12432 \mathrm{~N}, 1060 \mathrm{~m})$, Chas. $2207(27 / 7 / 2012,22.25849$ E, $41.1342 \mathrm{~N}, 1010 \mathrm{~m})$ - Common. In thermophilous deciduous stands and openings. On stony calcareous ground between 700 and $1400 \mathrm{~m}$. 
*Dipsacus laciniatus L. - H bienn, EA - Chas. 1592 (13/8/2011, 22.22706 E, 41.1112 $\mathrm{N}, 660 \mathrm{~m}$, grassland on damp ground) - Rare. In damp grasslands and damp places of forest roads, in the lowland areas.

Knautia ambigua Boiss. \& Orph. - H scap, Bk - Chas. 294 (10/9/2009, 22.25709 E, $41.1285 \mathrm{~N}, 880 \mathrm{~m}$, forest road in Quercus frainetto stand), Chas. 1716 (24/9/2011, 22.23437 E, $41.10969 \mathrm{~N}, 640 \mathrm{~m}$, dry grassland), MFG2: 356 - Common in grasslands at forest zone. Sporadic in grasslands at treeline and at forest roads.

* Knautia drymeia subsp. nympharum (Boiss. \& Heldr.) Ehrend. - H scap, Bk - Chas. $85(27 / 7 / 2009,22.1844$ E, 41.1335 N, 1190 m), Chas. 229 (24/8/2009, 22.21467 E, $41.12443 \mathrm{~N}, 1110 \mathrm{~m})$ - Common. In mesic beech stands and forest roads.

*Knautia integrifolia (L.) Bertol. - T scap, Me - Chas. 1358 (9/6/2011, 22.21812 E, $41.10692 \mathrm{~N}, 610 \mathrm{~m}$, forest road in dry grassland) - Sporadic. In forest roads and dry grasslands of lowland areas.

*Knautia magnifica Boiss. \& Orph. - H scap, Bk - Chas. 1560 (22/7/2011,22.17552 E, $41.15318 \mathrm{~N}, 1820 \mathrm{~m}$, grassy opening in subalpine shrubland) - Rare. In subalpine grasslands, on siliceous substrates.

*Lomelosia argentea (L.) Greuter \& Burdet - H bienn/H scap, EA - Chas. 313 (10/9/2009, 22.25484 E, 41.12793 N, 850 m, forest road in Quercus frainetto stand) - Sporadic. At forest roads and dry grasslands, of lowland areas.

*Lomelosia brachiata (Sm.) Greuter \& Burdet - T scap, EM - Chas. 1403 (16/6/2011, $22.2256 \mathrm{E}, 41.11253 \mathrm{~N}, 700 \mathrm{~m}$, open shrubland of Juniperus oxycedrus and thermophilous deciduous) - Sporadic. At forest roads, dry grasslands and open shrublands, of lowland areas.

Lomelosia crenata (Cyr.) Greuter \& Burdet - C suffr, Me - MFG2: 361.

Lomelosia graminifolia (L.) Greuter \& Burdet - C suffr, ME - MFG2: 363.

Scabiosa columbaria L. subsp. columbaria - H scap, EA - Chas. 2454 (14/7/2014, 22.23271 E, $41.1447 \mathrm{~N}, 2030 \mathrm{~m}$, subalpine shrubland), MFG2: 366 - Rare. In subalpine shrublands and grasslands, on stony ground.

Scabiosa triniifolia Friv. - H bienn, Bk - Chas. 171 (13/8/2009, 22.18554 E, 41.12244 $\mathrm{N}, 950 \mathrm{~m}$, grassy opening in beech stand), Chas. 207 (24/8/2009, 22.21374 E, $41.12378 \mathrm{~N}, 1120 \mathrm{~m}$, forest road in beech stand), MFG2: 368 - Common. Sparse grasslands on stony ground and forest roads, between 600 and $2000 \mathrm{~m}$.

\section{ERICACEAE}

Arctostaphylos uva-ursi (L.) Spreng. - C suffr/P caesp, Ct - Chas. 190 (24/8/2009, 22.21624 E, $41.12382 \mathrm{~N}, 1070 \mathrm{~m}$, open beech stand), Chas. 1746 (4/11/2011, $22.24701 \mathrm{E}, 41.14566 \mathrm{~N}, 1600 \mathrm{~m}$, low shrubland at treeline), Chas. 1867 $(16 / 5 / 2012,22.24427 \mathrm{E}, 41.15353 \mathrm{~N}, 1750 \mathrm{~m}$, shrubland above treeline), MFG1: 739 - Common in shrublands at above treeline. Rare in open beech stands. On calcareous substrates.

Bruckenthalia spiculifolia (Salisb.) Rchb. - C suffr, BA - Chas. 57 (20/7/2009, 22.21678 E, $41.1522 \mathrm{~N}, 1860 \mathrm{~m}$, subalpine shrubland), Chas. 165 (13/8/2009, 22.18554 E, 41.12244 N, $950 \mathrm{~m}$, opening in beech stand), Voliotis 1983: 164, MFG1: 738 - Common in subalpine shrublands on stony siliceous ground. Sporadic in open beech stands.

*Hypopitys monotropa Crantz - G par, Bo - Chas. 83 (27/7/2009, 22.1844 E, 41.1335 N, $1190 \mathrm{~m}$, beech stand) - Common in beech forests. Sporadic in mesic oak and thermophilous deciduous forests. 
Orthilia secunda (L.) House - H ros/C rept, Bo - Chas. 809 (26/6/2010, 22.22206 E, $41.12004 \mathrm{~N}, 900 \mathrm{~m})$, Chas. 1013 (3/4/2011, 22.22907 E, 41.13375 N, $1600 \mathrm{~m}$ ), Schuler 2004: map 44 - Common. Beech stands, mainly on shallow soils.

*Pyrola minor L. - H ros, Bo - Chas. 2049 (21/6/2012, 22.22042 E, 41.13641 N, 1400 $\mathrm{m}$, beech stand) - Rare.

Vaccinium myrtillus L. - C frut, ES - Chas. 146 (6/8/2009, 22.19142 E, 41.14685 N, $1560 \mathrm{~m}$, low shrubland at treeline), Chas. 1879 (19/5/2012, 22.22471 E, 41.15872 N, 1760 m, subalpine shrubland), MFG1: 742, Schuler 2004: map 45 - Common. Forms extensive subalpine mixed shrublands with Juniperus communis subsp. nana and other species of the Ericaceae family.

Vaccinium uliginosum subsp. microphyllum (Lange) Tolm - C frut, Bo - Chas. 257 (28/8/2009, 22.21989 E, 41.16045 N, 1920 m), Chas. 1856 (16/5/2012, 22.24073 E, $41.15558 \mathrm{~N}, 1710 \mathrm{~m}), \mathrm{MFG1}: 741$ - Common. In subalpine shrublands.

\section{EUPHORBIACEAE}

Euphorbia amygdaloides L. subsp. amygdaloides - C suffr/H caesp, EA - Chas. 91 (27/7/2009, 22.1844 E, 41.1335 N, 1190 m, beech stand), Chas. 120 (6/8/2009, 22.18389 E, $41.15205 \mathrm{~N}, 1590 \mathrm{~m}$, forest road in beech stand), Voliotis 1983: 164 Common. In forests all-over the mountain.

Euphorbia thessala (Formánek) Degen \& Dörfl. - C suffr, Bk - Chas. 845 (26/6/2010, 22.22398 E, $41.12844 \mathrm{~N}, 1440 \mathrm{~m}$, open shrubland of thermophilous deciduous and Juniperus oxycedrus on rocky slope), Chas. 1324 (2/6/2011, 22.2263 E, 41.13515 $\mathrm{N}, 1640 \mathrm{~m}$, sparse grassland at treeline on rocky ground), MFG1: 573 - Common. On rocky or stony calcareous ground at and above treeline with sparse vegetation. $1300-1900 \mathrm{~m}$.

*Euphorbia chamaesyce L. - T rept, ME - Chas. 2198 (27/7/2012, 22.26355 E, 41.11383 N, 630 m, roadside in dry grassland) - Rare. Roads of lowland areas.

*Euphorbia epithymoides L. - H caesp/G rhiz, Eu - Chas. 527 (2/5/2010, 22.18975 E, $41.12539 \mathrm{~N}, 1190 \mathrm{~m}$, forest road in beech stand), Chas. 872 (4/7/2010, 22.17565 E, $41.14408 \mathrm{~N}, 1370 \mathrm{~m}$, beech stand) - Sporadic. In beech forests and forest roads.

Euphorbia glabriflora Vis. - C suffr, BA - Chas. 1325 (2/6/2011, 22.23123 E, $41.13774 \mathrm{~N}, 1690 \mathrm{~m}$, subalpine sparse grassland on stony ground), MFG1: 568 Sporadic. Rocky and stony calcareous ground at and above treeline with sparse vegetation. Between 1500 and $1900 \mathrm{~m}$.

*Euphorbia helioscopia L. - T scap, Co - Chas. 1132 (1/5/2011, 22.20966 E, 41.11165 $\mathrm{N}, 800 \mathrm{~m}$, forest road in thermophilous deciduous and Juniperus oxycedrus stand) Rare. Disturbed sites, mainly forest roads, of lowland areas.

Euphorbia myrsinites L. - H caesp/C rept, ME - Chas. 441 (10/4/2010, 22.23015 E, 41.11602 N, 880 m, phrygana), Voliotis 1983: 164 - Common. Dry grasslands and phrygana on stony or rocky ground. Between 600 and $1500 \mathrm{~m}$.

Euphorbia oblongata Griseb. - H scap, BA - Voliotis 1983: 164.

*Euphorbia salicifolia Host - H scap, BC - Chas. 508 (2/5/2010, 22.20966 E, 41.11165 N, $800 \mathrm{~m}$, forest road in Pteridium thicket), Chas. 1133 (1/5/2011, 22.20894 E, $41.112 \mathrm{~N}, 810 \mathrm{~m}$, forest road in thermophilous deciduous and Juniperus oxycedrus stand), Chas. 1871 (19/5/2012, 22.2074 E, 41.11143 N, 810 m, Pteridium thicket) Sporadic. Forest roads, Pteridium thickets and shrublands of thermophilous deciduous and Juniperus oxycedrus, at low altitudes. 
*Euphorbia niciciana Borbás ex Novák - H scap, BA - Chas. 197 (24/8/2009, $22.19679 \mathrm{E}, 41.11622 \mathrm{~N}, 1040 \mathrm{~m}$, forest road in mixed thermophilous deciduous and beech stand) - Sporadic. In dry grasslands and forest roads, of lowland areas.

*Euphorbia taurinensis All. - T scap, EA - Chas. 241 (28/8/2009, 22.21245 E, $41.12746 \mathrm{~N}, 1290 \mathrm{~m}$, forest road in beech stand), Chas. 1144 (1/5/2011, $22.2164 \mathrm{E}$, $41.11356 \mathrm{~N}, 810 \mathrm{~m}$, phrygana) - Common. At forest roads, phrygana and open shrublands, of lowland areas.

\section{FABACEAE}

Anthyllis aurea Host - C suffr, Bk - Chas. 360 (15/9/2009, 22.25927 E, 41.16312 N, $1760 \mathrm{~m}$, subalpine sparse grassland), Chas. 397 (9/10/2009, 22.22749 E, 41.12192 N, $1200 \mathrm{~m}$, open shrubland of thermophilous deciduous and Juniperus oxycedrus), MFG1: 522, Schuler 2004: tab. 21 - Common. Stony calcareous places with sparse vegetation, between 1000 and $2050 \mathrm{~m}$.

Anthyllis vulneraria subsp. bulgarica - H scap, Bk - Chas. 580 (12/5/2010, 22.24246 E, $41.14107 \mathrm{~N}, 1530 \mathrm{~m}$, grassland on stony ground), Chas. $2080(25 / 6 / 2012$, 22.20799 E, 41.14684 N, $1980 \mathrm{~m}$, rocky opening in subalpine shrubland), Chas. $2348(1 / 7 / 2013,22.22534 \mathrm{E}, 41.13522 \mathrm{~N}, 1590 \mathrm{~m}$, rocky slope with sparse vegetation), Chas. 2381 (15/7/2013, $22.25267 \mathrm{E}, 41.16482 \mathrm{~N}, 1920 \mathrm{~m}$, sparse grassland on stony ground), MFG1: 524 - Common. Stony or rocky places at midaltitudes and above treeline, usually with sparse vegetation. Between 1400 and $2100 \mathrm{~m}$.

Anthyllis vulneraria subsp. pulchella (Vis.) Bornm. - H scap, Bk - Chas. 769 $(12 / 6 / 2010,22.17528 \mathrm{E}, 41.15759 \mathrm{~N}, 1890 \mathrm{~m}$, rocky opening in subalpine shrubland), Schuler 2004: tab. 21 (as A. vulnerarya subsp. scardica (Wettst.) Bergmeier) - Sporadic. Subalpine grasslands and openings in subalpine shrublands, on stony ground.

Anthyllis vulneraria subsp. rubriflora (DC.) Arcang. - H scap, Me - Chas. 1265 (28/5/2011, $22.20473 \mathrm{E}, 41.10896 \mathrm{~N}, 770 \mathrm{~m}$, dry grassland), Chas. 1883 (24/5/2012, $22.22723 \mathrm{E}, 41.11336 \mathrm{~N}, 760 \mathrm{~m}$, phrygana), Voliotis 1983: 164 (as $A$. vulneraria subsp. praepropera (A. Kern.) Bornm.) - Sporadic. Dry grasslands and phrygana, of lowland areas.

Astragalus angustifolius subsp. balcanicus Brullo, Giusso \& Musarella - C frut, Bk Chas. $576(12 / 5 / 2010,22.246 \mathrm{E}, 41.14229 \mathrm{~N}, 1590 \mathrm{~m}$, sparse grassland on stony ground), MFG1: 468 - Common. Stony or rocky places with sparse vegetation. 1300 - $2100 \mathrm{~m}$, occasionally descending to $600 \mathrm{~m}$.

Astragalus depressus L. - H ros, ME - Chas. 1332 (2/6/2011, 22.22875 E, 41.15005 N, $1690 \mathrm{~m}$, subalpine grassland on stony ground), Chas. 1830 (5/5/2012, 22.19009 E, $41.13909 \mathrm{~N}, 1490 \mathrm{~m}$, forest road in beech stand), MFG1: 462 - Rare.

*Astragalus glycyphyllos L. - H rept, ES - Chas. 26 (11/7/2009, 22.18 E, 41.12893 N, $1000 \mathrm{~m}$, beech stand), Chas. 816 (26/6/2010, $22.22406 \mathrm{E}, 41.11638 \mathrm{~N}, 800 \mathrm{~m}$, thermophilous deciduous stand) - Sporadic. Thermophilous deciduous stands, beech forests at the lower part of their distribution and oak forests.

*Astragalus hamosus L. - T scap, MS- Chas. 1284 (28/5/2011, 22.20684 E, 41.11034 N, 790 m, Pteridium thicket) - Rare.

Astragalus monspessulanus L. subsp. monspessulanus - $\mathrm{H}$ ros/H scap, Me - Chas. $487(25 / 4 / 2010,22.19838$ E, 41.10616 N, 710 m, open shrubland of Juniperus 
oxycedrus and thermophilous deciduous), Voliotis 1983: 164 - Common. Thermophilous deciduous forests and shrublands and oak forests.

*Bituminaria bituminosa (L.) C. H. Stirt. - H scap, ME - Chas. 1225 (25/5/2011, 22.22512 E, 41.11243 N, 700 m), Chas. 1349 (9/6/2011, 22.1953 E, 41.11404 N, $900 \mathrm{~m})$ - Sporadic. Thermophilous deciduous forests and shrublands.

Chamaecytisus austriacus L. (Link) - C suffr, BC - Chas. 1503 (2/7/2011, 22.27995 E, $41.14713 \mathrm{~N}, 920 \mathrm{~m}$, Juniperus oxycedrus - thermophilous deciduous shrubland within Quercus frainetto stand), Chas. 2208 (27/7/2012, 22.26385 E, 41.13878 N, $1070 \mathrm{~m}$, forest road in mixed beech - oak stand) - Rare.

Chamaecytisus hirsutus subsp. polytrichus (M. Bieb.) Ponert - C suffr, ME - Chas. $488(25 / 4 / 2010,22.19512$ E, 41.10755 N, 690 m, open Juniperus oxycedrus thermophilous deciduous shrubland), Chas. 522 (2/5/2010, 22.20281 E, 41.12686 N, $1370 \mathrm{~m}$, grassy opening in beech stand), Chas. $2036(18 / 6 / 2012,22.23847 \mathrm{E}$, $41.12092 \mathrm{~N}, 890 \mathrm{~m}$, thermophilous deciduous stand), Chas. 2097 (25/6/2012, 22.21386 E, $41.14567 \mathrm{~N}, 1820 \mathrm{~m}$, subalpine shrubland), Voliotis 1983: 164 (as $C$. hirsutus (L.) Link) - Common. Stony ground with herbaceous or shrubby vegetation, between 600 and $2000 \mathrm{~m}$.

*Colutea arborescens L. - P caesp, EA - Chas. 638 (19/5/2010, 22.2155 E, 41.11597 $\mathrm{N}, 980 \mathrm{~m}$ ) - Sporadic. In thermophilous deciduous forests and shrublands.

*Cytisus procumbens (Willd.) Spreng. - C suffr, BC - Chas. 1088 (22/4/2011, $22.23721 \mathrm{E}, 41.1191 \mathrm{~N}, 830 \mathrm{~m}$, rocky opening in thermophilous deciduous stand), Chas. $1779(22 / 4 / 2012,22.23536 \mathrm{E}, 41.117 \mathrm{~N}, 770 \mathrm{~m}$, rocky opening in thermophilous deciduous stand) - Rare.

*Dorycnium herbaceum Vill. - H caesp, ME - Chas. 708 (2/6/2010, 22.19336 E, $41.11536 \mathrm{~N}, 910 \mathrm{~m}$, forest road in thermophilous deciduous stand), Chas. 2007 (13/6/2012, 22.22911 E, $41.11925 \mathrm{~N}, 1070 \mathrm{~m}$, open shrubland of Juniperus oxycedrus and thermophilous deciduous) - Sporadic. Thermophilous deciduous shrublands, phrygana and forest roads of lowland areas, on stony ground.

*Dorycnium hirsutum (L.) Ser. - H scap/C suffr, Me - Chas. 709 (2/6/2010, 22.19679 $\mathrm{E}, 41.11622 \mathrm{~N}, 1030 \mathrm{~m}$, forest road in mixed beech - thermophilous deciduous stand) - Sporadic. Thermophilous deciduous shrublands, phrygana and forest roads of lowland areas, on stony ground.

*Genista carinalis Griseb. - C suffr, BA - Chas. 1498 (2/7/2011, 22.27737 E, 41.14583 N, $980 \mathrm{~m}$, Quercus frainetto stand) - Sporadic. Oak forests and thermophilous deciduous forests and shrublands.

Genista depressa M. Bieb. - C suffr, Bk - Chas. 702 (2/6/2010, 22.19887 E, 41.13175 $\mathrm{N}, 1630 \mathrm{~m}$, low shrubland at treeline), MFG1: 452 - Common. Grasslands and shrublands at and above treeline.

*Genista tinctoria L. - C suffr, ES - Chas. 389 (27/9/2009, 22.21237 E, 41.13016 N, $1320 \mathrm{~m}$, forest road in beech stand) - Rare.

*Hippocrepis ciliata Willd. - T scap, Me - Chas. 1177 (11/5/2011, 22.22418 E, 41.11187 N, 680 m, phrygana), Chas. 1917 (24/5/2012, 22.22059 E, 41.1128 N, $730 \mathrm{~m}$, phrygana) - Common. Phrygana and dry grasslands of lowland areas.

*Hippocrepis comosa L. - H caesp, Eu - Chas. 639 (19/5/2010, 22.21658 E, 41.11554 $\mathrm{N}, 930 \mathrm{~m}$, phrygana), Chas. $673(24 / 5 / 2010,22.25209 \mathrm{E}, 41.15977 \mathrm{~N}, 1750 \mathrm{~m}$, subalpine grassland on stony ground), Chas. 1533 (12/7/2011, $22.2298 \mathrm{E}, 41.1531$ $\mathrm{N}, 1850 \mathrm{~m}$, subalpine sparse grassland on stony ground), Chas. 1961 (4/6/2012, $22.24855 \mathrm{E}, 41.1181 \mathrm{~N}, 760 \mathrm{~m}$, open shrubland of Juniperus oxycedrus and 
thermophilous deciduous) - Common. Stony ground with herbaceous or shrubby vegetation. $600-2100 \mathrm{~m}$.

Hippocrepis emerus subsp. emeroides (Boiss. \& Spruner) Lassen - P caesp, EM Chas. 602 (19/5/2010, 22.21658 E, 41.11554 N, 920 m, shrubland of thermophilous deciduous and Juniperus oxycedrus), Voliotis 1983: 164 (as Coronilla emerus subsp. emeroides (Boiss. \& Spruner) Hayek) - Common. Oak forests, thermophilous deciduous forests and shrublands and forest roads, mainly on calcareous substrates.

*Lathyrus alpestris (Waldst. \& Kit.) Čelak. - H scap, Bk - Chas. 2217 (31/7/2012, 22.17379 E, $41.15531 \mathrm{~N}, 1930 \mathrm{~m}$, subalpine shrubland), Chas. 2265 (10/6/2013, 22.18215 E, $41.14617 \mathrm{~N}, 1530 \mathrm{~m}$, Pteridium thicket in beech forest) - Sporadic. Pteridium thickets at treeline and in beech forests and in shrublands at and above treeline.

*Lathyrus cicera L. - T scap, MS - Chas. 491 (25/4/2010, 22.19832 E, 41.10823 N, $770 \mathrm{~m}$, grassland within shrubland of thermophilous deciduous and Juniperus oxycedrus), Chas. 1167 (11/5/2011, 22.20668 E, $41.1116 \mathrm{~N}, 830 \mathrm{~m}$, forest road in thermophilous deciduous stand) - Common. Dry grasslands, open shrublands and forest roads, at lowland areas.

*Lathyrus grandiflorus Sibth. \& Sm. - H scap, BI - Chas. 2343 (26/6/2013, 22.20439 E, 41.13454 N, $1640 \mathrm{~m}$, Pteridium thicket in beech forest) - Rare.

*Lathyrus laxiflorus (Desf.) Kuntze - H scap, EM - Chas. 484 (25/4/2010, 22.19922 E, $41.11005 \mathrm{~N}, 820 \mathrm{~m}$, thermophilous deciduous stand), Chas. 1053 (16/4/2011, $22.25058 \mathrm{E}, 41.13155 \mathrm{~N}, 1000 \mathrm{~m}$, oak stand) - Common. Oak forests, thermophilous deciduous forests and shrublands and thermophilous beech forests.

*Lathyrus niger (L.) Bernh. subsp. niger - H scap, ME - Chas. 1438 (22/6/2011, $22.20333 \mathrm{E}, 41.11227 \mathrm{~N}, 880 \mathrm{~m}$, thermophilous deciduous stand), Chas. 1892 $(24 / 5 / 2012,22.20286 \mathrm{E}, 41.11217 \mathrm{~N}, 880 \mathrm{~m}$, thermophilous deciduous stand) Sporadic. Oak forests and thermophilous deciduous stands.

*Lathyrus nissolia L. - T scap, ME - Chas. 1244 (25/5/2011, 22.1961 E, 41.1199 N, $1130 \mathrm{~m}$, grassy opening in beech stand) - Sporadic. Openings and forest roads in beech forests.

*Lathyrus pratensis L. - H scap, Pt - Chas. 808 (26/6/2010, 22.21379 E, 41.12189 N, $1080 \mathrm{~m}$, forest road in mixed beech - thermophilous deciduous stand), Chas. 1691 (10/9/2011, 22.2587 E, 41.16221 N, 1700 m, subalpine grassland) - Common. Oak and beech forests, shrublands and grasslands at treeline and above treeline.

Lathyrus sativus L. - T scap, [?MS] - Voliotis 1983: 164.

Lathyrus sphaericus Retz. - T scap, EA - Voliotis 1983: 164.

*Lathyrus venetus (Mill.) Wohlf. - H scap, Eu - Chas. 1249 (25/5/2011, 22.21619 E, $41.12121 \mathrm{~N}, 1070 \mathrm{~m}$, beech stand) - Sporadic. In beech and oak forests.

*Lens nigricans (M. Bieb.) Godr. - T scap, Me - Chas. 1944 (30/5/2012, 22.19718 E, $41.111 \mathrm{~N}, 840 \mathrm{~m}$, open shrubland of thermophilous deciduous and Juniperus oxycedrus) - Rare.

Lotus corniculatus L. - H scap, EA - Chas. 17 (11/7/2009, 22.19274 E, 41.11643 N, $940 \mathrm{~m}$, forest road in thermophilous deciduous stand), Chas. $113(27 / 7 / 2009$, $22.18836 \mathrm{E}, 41.12816 \mathrm{~N}, 1190 \mathrm{~m}$, forest road in beech stand), Chas. 266 (28/8/2009, 22.21319 E, $41.14404 \mathrm{~N}, 1750 \mathrm{~m}$, subalpine shrubland), Chas. 758 (12/6/2010, 22.17528 E, $41.15759 \mathrm{~N}, 1900 \mathrm{~m}$, opening in subalpine shrubland), 
MFG1: 519 - Common. Stony ground with herbaceous vegetation or open shrublands. All-over the mountain.

*Lotus gebelia Vent. - H scap, IT - Chas. 1973 (4/6/2012, 22.20359 E, 41.11588 N, $980 \mathrm{~m}$, rocky margins of artificial lake) - Rare.

*Medicago lupulina L. - T scap, Ct - Chas. 2178 (11/7/2012, 22.17739 E, 41.13285 N, $1080 \mathrm{~m}$, forest road in beech stand) - Rare.

Medicago minima (L.) L. - T scap, Pt - Chas. 1232 (25/5/2011, 22.2208 E, 41.10921 $\mathrm{N}, 640 \mathrm{~m}$, dry grassland), Chas. 1873 (19/5/2012, 22.22252 E, $41.11167 \mathrm{~N}, 680 \mathrm{~m}$, forest road in shrubland of Juniperus oxycedrus and thermophilous deciduous), Voliotis 1983: 164 - Sporadic. Dry grasslands of lowland areas.

* Medicago monspeliaca (L.) Trautv. - T scap, MS - Chas. 1888 (24/5/2012, 22.23659 E, 41.10786 N, 630 m, phrygana with Juniperus oxycedrus) - Sporadic. Dry grasslands and phrygana, up to $900 \mathrm{~m}$.

*Medicago rigidula (L.) All. - T scap, MS - Chas. 1233 (25/5/2011, 22.2208 E, 41.10921 N, $640 \mathrm{~m}$ ) - Sporadic. Dry grasslands, up to $900 \mathrm{~m}$.

* Medicago sativa subsp. falcata (L.) Arcang. - Hscap, EA - Chas. 404 (9/10/2009, $22.22863 \mathrm{E}, 41.12356 \mathrm{~N}, 1270 \mathrm{~m}$, grassland within shrubland of Juniperus oxycedrus and thermophilous deciduous), Chas. 2008 (13/6/2012, 22.23007 E, $41.11827 \mathrm{~N}, 1010 \mathrm{~m}$, grassland within shrubland of Juniperus oxycedrus and thermophilous deciduous) - Common. Grasslands and open shrublands of lowland areas.

*Melilotus albus Medik. - T scap/H bienn, EA - Chas. 159 (13/8/2009, 22.21595 E, $41.11238 \mathrm{~N}, 760 \mathrm{~m}$, forest road in shrubland of thermophilous deciduous and Juniperus oxycedrus) - Sporadic. Forest roads up to $1300 \mathrm{~m}$.

*Melilotus neapolitanus Ten. - T scap, EA - Chas. 1195 (20/5/2011, 22.22441 E, 41.11224 N, 690 m, dry grassland), Chas. 1231 (25/5/2011, 22.20407 E, $41.1112 \mathrm{~N}$, $820 \mathrm{~m}$, grassland) - Sporadic. Roads and dry grasslands, up to $1000 \mathrm{~m}$.

*Onobrychis alba (Waldst. \& Kit.) Desv. subsp. alba - H scap, BI - Chas. 637 (19/5/2010, 22.21658 E, 41.11554 N, 920 m, phrygana with Juniperus oxycedrus), Chas. 775 (12/6/2010, 22.22204 E, 41.11193 N, 690 m, phrygana with Juniperus oxycedrus), BI - Chas. 1188 (20/5/2011, 22.22748 E, 41.11346 N, 760 m, phrygana) - Sporadic. Phrygana and roads, at lowland areas.

Onobrychis alba subsp. pentelica (Hausskn.) Nyman - H scap, BI - Chas. 683 (24/5/2010, 22.25245 E, 41.15978 N, 1730 m), Chas. 2298 (18/6/2013, 22.24251 E, $41.13909 \mathrm{~N}, 1450 \mathrm{~m})$, Chas. 2345 (1/7/2013, $22.22925 \mathrm{E}, 41.13591 \mathrm{~N}, 1620 \mathrm{~m})$, Chas. 2382 (15/7/2013, 22.2477 E, 41.16367 N, 1980 m), MFG1: 534 - Common. Grasslands above treeline and at mid-altitudes, on stony ground.

*Onobrychis arenaria subsp. lasiostachya (Boiss.) Hayek - H scap, BA - Chas. 2281 $(14 / 6 / 2013,22.2687$ E, 41.16272 N, 1570 m, grassland) - Rare.

*Onobrychis caput-galli Lam. - T scap, Me - Chas. 1187 (20/5/2011, 22.22418 E, 41.11187 N, $680 \mathrm{~m}$ ) - Sporadic. Dry grasslands up to $900 \mathrm{~m}$.

Onobrychis degenii Dörfl. - H scap, Bk - Voliotis 1983: 164.

*Onobrychis montana subsp. scardica (Griseb.) P. W. Ball - H scap, Bk - Chas. 577 $(12 / 5 / 2010,22.24605$ E, 41.14049 N, 1490 m), Chas. 1540 (12/7/2011, 22.23092 E, 41.15454 N, $1850 \mathrm{~m})$, Chas. $2282(14 / 6 / 2013,22.26275$ E, $41.16245 \mathrm{~N}, 1700 \mathrm{~m})$ Sporadic. Grasslands above treeline and at mid-altitudes, on stony ground.

*Onobrychis pindicola Hausskn. - H scap, Bk - Chas. 713 (2/6/2010, 22.21583 E, $41.11247 \mathrm{~N}, 760 \mathrm{~m}$, stony roadside in shrubland of thermophilous deciduous and 
Juniperus oxycedrus), Chas. 774 (12/6/2010, 22.21977 E, 41.11199 N, 700 m, stony roadside in shrubland of thermophilous deciduous and Juniperus oxycedrus), Chas. 2296 (18/6/2013, 22.23794 E, 41.13641 N, 1490 m, grassland) - Sporadic. Grasslands and roadsides, up to $1600 \mathrm{~m}$.

*Ononis pusilla L. - H scap, Me - Chas. 1433 (16/6/2011, 22.22484 E, 41.11731 N, $840 \mathrm{~m}$, shrubland of thermophilous deciduous and Juniperus oxycedrus), Chas. 1907 (24/5/2012, 22.23659 E, 41.10786 N, 620 m, phrygana with Juniperus oxycedrus) - Sporadic. Dry grasslands, phrygana and shrublands, up to $1000 \mathrm{~m}$.

*Ononis spinosa subsp. leiosperma (Boiss.) Širj. - C suffr, EM - Chas. 318 (10/9/2009, 22.26185 E, $41.12866 \mathrm{~N}, 790 \mathrm{~m}$, forest road in oak forest) - Sporadic. At forest roads and dry grasslands, of lowland areas.

*Oxytropis pilosa (L.) DC. - H scap, ES - Chas. 205 (24/8/2009, 22.21402 E, 41.12226 $\mathrm{N}, 1080 \mathrm{~m}$, forest road in mixed beech - thermophilous deciduous stand), Chas. 806 $(26 / 6 / 2010,22.21414 \mathrm{E}, 41.12199 \mathrm{~N}, 1080 \mathrm{~m}$, forest road in mixed beech thermophilous deciduous stand) - Rare. At the collection localities and at "Vrachi" in grasslands on rocky or stony ground.

*Robinia pseudoacacia L. - P caesp/P scap, [N-Am.] - Chas. 2042 (21/6/2012, 22.20443 E, $41.12445 \mathrm{~N}, 1210 \mathrm{~m}$, forest road in beech stand) - Rare. Road embankments of lowland areas.

Securigera parviflora(Desv.) Lassen - T scap, EM - Voliotis 1983: 164 (as Coronilla rostrata Boiss. \& Spruner).

*Securigera varia (L.) Lassen - H scap, EA - Chas. 870 (4/7/2010, 22.17565 E, $41.14408 \mathrm{~N}, 1370 \mathrm{~m}$, beech forest - Sporadic. In beech and oak forests, mainly in open stands.

*Spartium junceum L. - P caesp, Me - Chas. 1461 (27/6/2011, 22.18851 E, 41.11843 $\mathrm{N}, 980 \mathrm{~m}$, roadside in mixed beech - thermophilous deciduous stand) - Rare. Roadsides of lowland areas.

*Trifolium alpestre L. - G rhiz, EA - Chas. 67 (20/7/2009, 22.22555 E, 41.15414 N, $1830 \mathrm{~m}$, subalpine grassland), Chas. 828 (26/6/2010, $22.2213 \mathrm{E}, 41.11705 \mathrm{~N}, 840$ $\mathrm{m}$, thermophilous deciduous shrubland), Chas. $1582(22 / 7 / 2011,22.17672 \mathrm{E}$, 41.15249 N, $1770 \mathrm{~m}$, subalpine shrubland), Chas. 1949 (30/5/2012, 22.19652 E, $41.10892 \mathrm{~N}, 790 \mathrm{~m}$, open shrubland of thermophilous deciduous and Juniperus oxycedrus) - Common. Forests, shrublands and grasslands, between 700 and 2000 m.

*Trifolium angustifolium L. - T scap, EA - Chas. 2146 (6/7/2012, 22.28378 E, 41.14205 N, 780 m, forest road in Quercus frainetto stand), Chas. 2441 (28/6/2014, 22.20244 E, 41.1101 N, 830 m, dry grassland) - Sporadic. Dry grasslands and forest roads, up to $900 \mathrm{~m}$.

*Trifolium arvense L. - T scap, $\mathrm{Pt}$ - Chas. 773 (12/6/2010, 22.21168 E, $41.11186 \mathrm{~N}$, $790 \mathrm{~m}$, forest road in shrubland of Juniperus oxycedrus and thermophilous deciduous) - Sporadic. Dry grasslands and forest roads of lowland areas.

*Trifolium aureum Pollich - T scap, ES - Chas. 2357 (1/7/2013, 22.2148 E, 41.13308 N, $1360 \mathrm{~m}$, forest road in beech stand), Chas. $2446(6 / 7 / 2014,22.18803 \mathrm{E}$, $41.13608 \mathrm{~N}, 1360 \mathrm{~m}$, forest road in beech stand) - Rare.

*Trifolium badium Schreb. - H scap, Eu - Chas. 275 (28/8/2009, 22.21988 E, $41.16081 \mathrm{~N}, 1910 \mathrm{~m}$, above treeline fen), Chas. $759(12 / 6 / 2010,22.20086 \mathrm{E}$, $41.15907 \mathrm{~N}, 1930 \mathrm{~m}$, streambank above treeline), Chas. $2136(30 / 6 / 2012,22.22148$ 
E, 41.15903 N, 1850 m, above treeline fen) - Sporadic. Fens and other damp places above treeline.

*Trifolium campestre Schreb. - T scap, EA - Chas. 23 (11/7/2009, 22.2049 E, 41.11167 $\mathrm{N}, 840 \mathrm{~m}$, forest road in thermophilous deciduous stand), Chas. 867 (4/7/2010, $22.18458 \mathrm{E}, 41.13585 \mathrm{~N}, 1280 \mathrm{~m}$, grassland on stony ground within beech stand) Sporadic. Forest roads and grasslands up to the treeline.

*Trifolium cherleri L. - Tscap, Me - Chas. 1989 (4/6/2012, 22.25307 E, 41.10934 N, $630 \mathrm{~m}$, dry grassland) - Rare.

*Trifolium dalmaticum Vis. - T scap, Bk - Chas. 1303 (2/6/2011, 22.22255 E, $41.11068 \mathrm{~N}, 670 \mathrm{~m})$, Chas. 1363 (9/6/2011, 22.22255 E, $41.11068 \mathrm{~N}, 670 \mathrm{~m})$ Sporadic. Dry grasslands, between 600 and $850 \mathrm{~m}$. Occasionally abundant.

*Trifolium diffusum Ehrh. - T scap, EA - Chas. 2439 (28/6/2014, 22.20208 E, 41.11 N, $830 \mathrm{~m}$, dry grassland) - Rare.

Trifolium heldreichianum (Gibelli \& Belli) Hausskn. - G rhiz, BA - Chas. 2433 (19/6/2014, 22.29163 E, 41.14721 N, 820 m), MFG1: 512 - Rare, but occasionally abundant. In oak forests on siliceous ground.

*Trifolium hirtum All. - T scap, Me - Chas. 1950 (30/5/2012, 22.19745 E, 41.10743 N, 760 m, open Juniperus oxycedrus shrubland) - Sporadic. Phrygana and open shrublands up to $900 \mathrm{~m}$.

*Trifolium hybridum L. subsp. hybridum - H caesp, EA - Chas. 1570 (22/7/2011, $22.17604 \mathrm{E}, 41.15589 \mathrm{~N}, 1830 \mathrm{~m}$, streambank above treeline), Chas. 2137 (30/6/2012, 22.22148 E, 41.15903 N, 1850 m, above treeline fen) - Sporadic. Fens and other damp places above treeline.

*Trifolium incarnatum L. - T scap, ME - Chas. 1266 (28/5/2011, 22.2031 E, 41.10776 N, 740 m, dry grassland) - Rare.

*Trifolium medium subsp. balcanicum Velen. - G rhiz, Bk - Chas. 779 (12/6/2010, $22.19439 \mathrm{E}, 41.14735 \mathrm{~N}, 1640 \mathrm{~m}$, shrubland of Juniperus communis subsp. nana at treeline), Chas. 1314 (2/6/2011, 22.21825 E, 41.14178 N, $1590 \mathrm{~m}$, shrubland of Juniperus communis subsp. nana at treeline), Chas. 1529 (12/7/2011, 22.2096 E, $41.14011 \mathrm{~N}, 1690 \mathrm{~m}$, roadside in beech stand at treeline) - Sporadic. Roads and shrublands at and above treeline. Between 1200 and $1900 \mathrm{~m}$.

Trifolium noricum Wulfen - H caesp, BI - Chas. 2378 (15/7/2013, 22.24285 E, $41.16215 \mathrm{~N}, 1820 \mathrm{~m}$, subalpine grassland), MFG1: 511 - Rare.

*Trifolium ochroleucon Huds. - H scap, ME - Chas. 877 (4/7/2010, 22.1769 E, $41.14644 \mathrm{~N}, 1450 \mathrm{~m}$, forest road in beech stand) - Sporadic in oak forests. Rare in forest roads of beech stands and in thermophilous beech stands.

Trifolium pallescens Schreb. - H caesp, Eu - Chas. 914 (1/8/2010, 22.20629 E, $41.16096 \mathrm{~N}, 2150 \mathrm{~m}$, subalpine grassland on stony ground), Chas. $1186(16 / 5 / 2011$, $22.19274 \mathrm{E}, 41.11643 \mathrm{~N}, 950 \mathrm{~m}$, forest road in thermophilous deciduous stand), Chas. $1255(25 / 5 / 2011,22.2156 \mathrm{E}, 41.12111 \mathrm{~N}, 1090 \mathrm{~m}$, grassy opening in thermophilous deciduous stand), Chas. 1569 (22/7/2011, 22.17604 E, 41.15589 N, $1830 \mathrm{~m}$, grassy opening in subalpine shrubland), MFG1: 505 - Common in subalpine grasslands and shrublands, mainly on siliceous ground. Rare in forest roads of low altitudes, up to $950 \mathrm{~m}$.

*Trifolium physodes M. Bieb. - H scap, Me - Chas. 1974 (4/6/2012, 22.25456 E, $41.11171 \mathrm{~N}, 650 \mathrm{~m}$, dry grassland) - Rare.

Trifolium pignantii Fauché \& Chaub. - G rhiz, Bk - Chas. 519 (2/5/2010, 22.21162 E, $41.12276 \mathrm{~N}, 1120 \mathrm{~m}$, mixed beech - thermophilous deciduous stand), Chas. 657 
$(24 / 5 / 2010,22.24492 \mathrm{E}, 41.15597 \mathrm{~N}, 1640 \mathrm{~m}$, beech stand at treeline), Chas. 1851 $(16 / 5 / 2012,22.21226 \mathrm{E}, 41.12997 \mathrm{~N}, 1330 \mathrm{~m}$, forest road in beech stand), Voliotis 1983: 164 - Common. Oak forests, thermophilous deciduous stands and thermophilous beech stands.

*Trifolium pratense L. - H scap, EA - Chas. 58 (20/7/2009, 22.21261 E, 41.14349 N, $1730 \mathrm{~m}$, forest road above treeline), Chas. $68(20 / 7 / 2009,22.21485 \mathrm{E}, 41.13551 \mathrm{~N}$, $1440 \mathrm{~m}$, forest road in beech stand), Chas. 282 (28/8/2009, $22.20834 \mathrm{E}, 41.13829$ $\mathrm{N}, 1690 \mathrm{~m}$, forest road in beech stand at treeline), Chas. $1468(27 / 6 / 2011,22.20764$ E, $41.11153 \mathrm{~N}, 820 \mathrm{~m}$, forest road in thermophilous deciduous stand) - Sporadic. Oak forests, thermophilous deciduous stands, thermophilous beech stands and forest roads.

*Trifolium purpureum Loisel. - T scap, Me - Chas. 2119 (30/6/2012, 22.1931 E, $41.11625 \mathrm{~N}, 950 \mathrm{~m}$, forest road in thermophilous deciduous stand) - Rare.

*Trifolium repens L. - H rept, Pt - Chas. 69 (20/7/2009, 22.21765 E, 41.14663 N, 1790 $\mathrm{m}$, road above treeline) - Sporadic. Grasslands and roads from low to high altitudes.

Trigonella caerulea subsp. procumbens (Besser) Thell. - T scap, EA - Chas. 2151 (6/7/2012, 22.28378 E, 41.14205 N, 780 m, forest road in Quercus frainetto stand), Chasapis et al. 2019 - Rare.

*Trigonella corniculata subsp. balansae (Boiss. \&Reut.) Lassen - T scap, EM - Chas. $1872(19 / 5 / 2012,22.20835 \mathrm{E}, 41.11172 \mathrm{~N}, 810 \mathrm{~m}$, forest road in thermophilous deciduous stand) - Sporadic. In forest roads and dry grasslands up to $900 \mathrm{~m}$.

Vicia angustifolia L. - T scap, Pt - Chas. 1165 (11/5/2011, 22.19614 E, 41.11387 N, $900 \mathrm{~m}$, forest road in thermophilous deciduous stand), Chas. $1302(2 / 6 / 2011$, 22.22255 E, $41.11068 \mathrm{~N}, 670 \mathrm{~m}$, dry grassland), Voliotis 1983: 164 (as V. nigra subsp. angustifolia (L.) Ehrh.) - Sporadic. Roads, phrygana, open shrublands and dry grasslands of lowland areas.

*Vicia bithynica (L.) L. - T scap, Me - Chas. 1282 (28/5/2011, 22.20717 E, 41.11107 $\mathrm{N}, 800 \mathrm{~m}$, Pteridium thicket) - Rare.

*Vicia cassubica L. - H scap, EA - Chas. 2258 (1/6/2013, 22.27889 E, 41.13748 N, $820 \mathrm{~m}$, Quercus frainetto stand) - Rare.

*Vicia cracca subsp. incana (Gouan) Rouy - H scap, Ct - Chas. 711 (2/6/2010, $22.19336 \mathrm{E}, 41.11536 \mathrm{~N}, 920 \mathrm{~m}$, forest road in thermophilous deciduous stand), Chas. 1245 (25/5/2011, 22.1967 E, 41.11964 N, 1130 m, forest road in mixed beech - thermophilous deciduous stand) - Sporadic. Openings and forest roads of oak forests, thermophilous deciduous stands and thermophilous beech stands.

*Vicia grandiflora Scop. - T scap, ME - Chas. 1279 (28/5/2011, 22.19908 E, 41.11977 $\mathrm{N}, 1110 \mathrm{~m}$, forest road in mixed beech - thermophilous deciduous stand), Chas. 2255 (1/6/2013, 22.27889 E, 41.13748 N, 820 m, Quercus frainetto stand) Sporadic. Open oak, beech and thermophilous deciduous stands and forest roads and openings.

*Vicia hirsuta (L.) Gray - T scap, Pt - Chas. 1166 (11/5/2011, 22.22418 E, 41.11187 N, $680 \mathrm{~m}$, dry grassland), Chas. $1884(24 / 5 / 2012,22.21027 \mathrm{E}, 41.11553 \mathrm{~N}, 910 \mathrm{~m}$, thermophilous deciduous stand) - Sporadic. Open forests and shrublands and grasslands of lowland areas.

Vicia lathyroides L. - T scap, ME - Chas. 1114 (26/4/2011, 22.20094 E, 41.10836 N, $770 \mathrm{~m}$, open Juniperus oxycedrus - thermophilous deciduous shrubland), Chas. $1164(11 / 5 / 2011,22.19758 \mathrm{E}, 41.11344 \mathrm{~N}, 900 \mathrm{~m}$, forest road in thermophilous 
deciduous stand), Voliotis 1983: 164 - Sporadic. Grasslands, open shrublands and forest roads, of lowland areas.

*Vicia narbonensis L. - T scap, MS - Chas. 666 (24/5/2010, 22.26465 E, 41.14447 N, $1170 \mathrm{~m}$, forest road in mixed beech - oak stand) - Rare.

*Vicia sepium L. - H scap, ES - Chas. 698 (2/6/2010, 22.19567 E, 41.1353 N, 1600 m, beech stand), Chas. 2269 (10/6/2013, 22.17219 E, 41.14448 N, $1450 \mathrm{~m}$, beech stand) - Sporadic. In beech stands and in Pteridium thickets at mid-altitudes.

*Vicia tenuifolia subsp. dalmatica (A. Kern.) Greuter - H scap, ME - Chas. 690 $(2 / 6 / 2010,22.18404 \mathrm{E}, 41.12503 \mathrm{~N}, 1010 \mathrm{~m}$, forest road in beech stand), Chas. 1939 (30/5/2012, 22.19278 E, 41.1149 N, 880 m, thermophilous deciduous stand) Common. Thermophilous beech forests, oak forests, thermophilous deciduous stands and forest roads, up to $1400 \mathrm{~m}$.

*Vicia tetrasperma (L.) Schreb. - T scap, Pt - Chas. 1457 (22/6/2011, 22.21024 E, 41.11555 N, 910 m, thermophilous deciduous stand) - Sporadic. Thermophilous deciduous and oak forests.

*Vicia villosa subsp. varia (Host) Corb. - T scap, ME - Chas. 400 (9/10/2009, 22.22873 E, $41.11987 \mathrm{~N}, 1100 \mathrm{~m}$, grassland within thermophilous deciduous Juniperus oxycedrus shrubland), Chas. 716 (2/6/2010, 22.19442 E, 41.12006 N, $1130 \mathrm{~m}$, forest road in beech stand) - Sporadic. Forests, shrublands, grasslands and forest roads of lowland areas.

*Vicia villosa Roth subsp. villosa - T scap, EA - Chas. 1926 (30/5/2012, 22.19779 E, $41.10597 \mathrm{~N}, 710 \mathrm{~m}$, grassy opening in shrubland of Juniperus oxycedrus and thermophilous deciduous) - Rare.

\section{FAGACEAE}

*Castanea sativa Mill. - P scap, Eu - Chas. 2209 (27/7/2012, 22.2551 E, 41.1273 N, $830 \mathrm{~m}$, beech stand within oak forest) - Rare.

Fagus sylvatica L. subsp. sylvatica - P scap, Eu - Chas. 857 (4/7/2010, 22.17804 E, $41.1352 \mathrm{~N}, 1130 \mathrm{~m}$, beech stand), Chas. 1874 (19/5/2012, 22.19323 E, $41.11572 \mathrm{~N}$, $940 \mathrm{~m}$, mixed beech - thermophilous deciduous stand), Voliotis 1983: 164, Parent 2005: 198, FH1: map 56 - Common. Forming extensive forests. 700 - $1800 \mathrm{~m}$.

*Quercus coccifera L. - P caesp, Me - Chas. 485 (25/4/2010, 22.19682 E, 41.11082 N, $840 \mathrm{~m}$ ) - Rare. In shrublands between 600 and $850 \mathrm{~m}$.

*Quercus frainetto Ten. - P scap, BA - Chas. 283 (10/9/2009, 22.26008 E, 41.12827 N, $810 \mathrm{~m})$ - Common. Forming forests in the southeast part of the mountain. 650 $1300 \mathrm{~m}$.

*Quercus petraea subsp. polycarpa (Schur) Soó - P scap, ME - Chas. 295 (10/9/2009, 22.25179 E, 41.13085 N, 970 m, mixed stand with Quercus frainetto) - Sporadic. Forming thickets within Quercus frainetto forests or scattered in thermophilous beech and Quercus frainetto forests.

Quercus pubescens Willd. - P scap, ME - Chas. 163 (13/8/2009, 22.21772 E, 41.11313 $\mathrm{N}, 760 \mathrm{~m}$, thermophilous deciduous stand), FH1: map 72 - Common. Forming thickets or in mixture with other thermophilous deciduous trees and shrubs. Mainly on calcareous substrates. $600-1500 \mathrm{~m}$.

\section{FUMARIACEAE}

Corydalis cava (L.) Schweigg. \& Körte - G bulb, EA - Chas. 1150 (6/5/2011, 22.25066 E, $41.15101 \mathrm{~N}, 1730 \mathrm{~m}$, beech stand at treeline), Chas. $1772(19 / 4 / 2012,22.21411$ 
E, $41.13631 \mathrm{~N}, 1470 \mathrm{~m}$, beech stand), Voliotis 1983: 166 - Sporadic. Beech stands, Pteridium thickets and grasslands, between 1400 and $1900 \mathrm{~m}$.

*Corydalis solida subsp. incisa Lidén - G bulb, Bk - Chas. 557 (8/5/2010, 22.244 E, $41.15938 \mathrm{~N}, 1820 \mathrm{~m}$, subalpine grassland), Chas. 1790 (25/4/2012, 22.25414 E, $41.15908 \mathrm{~N}, 1660 \mathrm{~m}$, grassland at treeline) - Sporadic. Grasslands and Pteridium thickets, between 1400 and $2000 \mathrm{~m}$.

*Corydalis solida (L.) Clairv. subsp. solida - G bulb, EA - Chas. 1002 (31/3/2011, $22.27976 \mathrm{E}, 41.1455 \mathrm{~N}, 870 \mathrm{~m}$, thermophilous deciduous stand), Chas. 2241 (11/4/2013, 22.27988 E, $41.14524 \mathrm{~N}, 860 \mathrm{~m}$, thermophilous deciduous stand) Rare.

*Fumaria kralikii Jord. - T scap, Me - Chas. 1098 (26/4/2011, 22.19809 E, 41.10372 $\mathrm{N}, 610 \mathrm{~m}$, open shrubland of thermophilous deciduous and Juniperus oxycedrus) Rare.

*Fumaria officinalis L. subsp. officinalis - T scap, Pt - Chas. 1808 (2/5/2012, 22.218 E, $41.107 \mathrm{~N}, 620 \mathrm{~m}$, dry grassland) - Rare.

?Fumaria officinalis subsp. wirtgenii (W. D. J. Koch) Arcang. - T scap, Eu - Voliotis 1983: 166.

*Fumaria petteri Rchb. - T scap, ME - Chas. 1074 (22/4/2011, 22.23695 E, 41.12441 $\mathrm{N}, 1070 \mathrm{~m}$, grassy opening in thermophilous deciduous stand), Chas. 1130 $(1 / 5 / 2011,22.22331 \mathrm{E}, 41.11781 \mathrm{~N}, 840 \mathrm{~m}$, grassy opening in thermophilous deciduous stand) - Sporadic. Grasslands of lowland areas.

*Fumaria rostellata Knaf - T scap, BC - Chas. 506 (2/5/2010, 22.19337 E, 41.11518 $\mathrm{N}, 910 \mathrm{~m}$, road in thermophilous deciduous stand), Chas. $1217(20 / 5 / 2011$, $22.22834 \mathrm{E}, 41.12122 \mathrm{~N}, 1170 \mathrm{~m}$, grassland within shrubland of thermophilous deciduous and Juniperus oxycedrus), Chas. 1770 (12/4/2012, 22.20148 E, 41.11017 N, 820 m, Pteridium thicket) - Sporadic. Grasslands, Pteridium thickets, open shrublands and roads, at lowland areas.

*Fumaria vaillantii Loisel. - T scap, EA - Chas. 1807 (2/5/2012, 22.21984 E, 41.10965 N, 660 m, dry grassland) - Rare.

\section{GENTIANACEAE}

*Blackstonia perfoliata (L.) Huds. subsp. perfoliata - T scap, ME - Chas. 213 $(24 / 8 / 2009,22.21374 \mathrm{E}, 41.12378 \mathrm{~N}, 1130 \mathrm{~m}$, forest road in mixed beech thermophilous deciduous stand), Chas. 810 (26/6/2010, 22.22203 E, $41.1167 \mathrm{~N}$, $840 \mathrm{~m}$, grassy opening in thermophilous deciduous stand) - Sporadic. Grasslands, phrygana and forest roads, at lowland areas.

*Centaurium erythraea subsp. rhodense (Boiss. \&Reut.) Melderis - T scap/H bienn, Me - Chas. $1467(27 / 6 / 2011,22.20787$ E, $41.11162 \mathrm{~N}, 810 \mathrm{~m}$, forest road in thermophilous deciduous stand), Chas. 2173 (11/7/2012, 22.18808 E, 41.12122 N, $1010 \mathrm{~m}$, forest road in beech stand) - Sporadic. Forest roads, grasslands and phrygana, at lowland areas.

*Centaurium pulchellum (Sw.) Druce - T scap, EA - Chas. 2448 (6/7/2014, 22.21409 E, 41.11082 N, 750 m, dry grassland) - Sporadic. Dry grasslands of lowland areas.

Gentiana asclepiadea L. - H scap, BC - Chas. 239 (28/8/2009, 22.21319 E, 41.14404 $\mathrm{N}, 1750 \mathrm{~m}$, damp place in subalpine shrubland), MFG2: 4 - Sporadic. Damp places at and above treeline and ditches by roadsides in beech forests. $1400-1800 \mathrm{~m}$.

*Gentiana punctata L. - H scap, BC - Chas. 311 (10/9/2009, 22.25447 E, 41.13756 N, $1210 \mathrm{~m}$, forest road in beech stand) - Rare. 
Gentiana verna subsp. balcanica N. M. Pritch. - H ros, BA - Chas. 379 (27/9/2009, 22.23259 E, $41.14488 \mathrm{~N}, 2010 \mathrm{~m}$, subalpine grassland on stony ground), Chas. 552 $(8 / 5 / 2010,22.24032 \mathrm{E}, 41.15437 \mathrm{~N}, 1780 \mathrm{~m}$, grassy opening in subalpine shrubland), Chas. 1825 (2/5/2012, $22.22206 \mathrm{E}, 41.14625 \mathrm{~N}, 1770 \mathrm{~m}$, subalpine grassland), MFG2: 6, Schuler 2004: tab. 21 -Common. In subalpine grasslands and open shrublands, mainly on stony ground. Between 1700 and $2180 \mathrm{~m}$.

Gentiana verna L. subsp. verna - H ros, EA - Voliotis 1983: 165.

Gentianella bulgarica (Velen.) Holub - T scap/H bienn, Bk - Chas. 256 (28/8/2009, $22.21659 \mathrm{E}, 41.14616 \mathrm{~N}, 1780 \mathrm{~m}$, open subalpine shrubland), Chas. 426 (31/10/2009, 22.21109 E, 41.15536 N, 2060 m, subalpine grassland), MFG2: 8, Sporadic. In subalpine grasslands and open shrublands, between 1700 and $2180 \mathrm{~m}$.

Gentianella crispata (Vis.) Holub - H bienn, BI - Chas. 377 (27/9/2009, 22.23095 E, 41.14386 N, 1990 m), Chas. 1623 (18/8/2011, 22.22839 E, 41.14599 N, $1890 \mathrm{~m})$, MFG2: 8 - Sporadic. Subalpine grasslands on stony calcareous ground. Between 1800 and $2050 \mathrm{~m}$.

Gentianopsis ciliata (L.) Ma - H scap, ME - Chas. 376 (27/9/2009, 22.21367 E, $41.14387 \mathrm{~N}, 1760 \mathrm{~m}$, road above treeline), Chas. 1698 (13/9/2011, 22.23539 E, $41.15591 \mathrm{~N}, 1820 \mathrm{~m}$, subalpine grassland on stony ground), Chasapis et al. 2019 Rare.

\section{GERANIACEAE}

Erodium cicutarium (L.) L'Hér. - T scap, Ct - Chas. 976 (13/3/2011, 22.21014 E, 41.11148 N, 800 m, grassland), Chas. 999 (28/3/2011, 22.19807 E, 41.10435 N, $650 \mathrm{~m}$, grassy opening in shrubland of thermophilous deciduous and Juniperus oxycedrus), Voliotis 1983: 165 - Common. Grasslands, open shrublands, phrygana and forest roads, at lowland areas.

*Geranium columbinum L. - T scap, EA - Chas. 1211 (20/5/2011, 22.22876 E, 41.11456 N, 820 m, phrygana) - Rare.

*Geranium divaricatum Ehrh. - T scap, EA - Chas. 1960 (4/6/2012, 22.20377 E, $41.11786 \mathrm{~N}, 980 \mathrm{~m}$, grassy opening in thermophilous deciduous stand on damp ground) - Rare.

*Geranium lucidum L. - T scap, EA - Chas. 465 (17/4/2010, 22.22106 E, 41.11732 N, $840 \mathrm{~m}$, thermophilous deciduous stand) - Common. Thermophilous deciduous stands and oak forests, mainly on calcareous substrates.

*Geranium macrorrhizum L. - G rhiz, ME - Chas. 525 (2/5/2010, 22.19723 E, $41.12181 \mathrm{~N}, 1220 \mathrm{~m}$, opening of beech stand on rocky ground), Chas. 662 $(24 / 5 / 2010,22.2657 \mathrm{E}, 41.15457 \mathrm{~N}, 1220 \mathrm{~m}$, rocky streambank in beech stand) Sporadic. Rocky and stony places in beech stands, between 1100 and $1700 \mathrm{~m}$.

*Geranium molle L. - T scap, Pt - Chas. 1040 (8/4/2011, 22.19996 E, 41.10483 N, 670 $\mathrm{m}$, grassy opening in shrubland of thermophilous deciduous and Juniperus oxycedrus) - Sporadic. Roads and dry grasslands, up to $900 \mathrm{~m}$.

*Geranium purpureum Vill. - T scap, Me - Chas. 1214 (20/5/2011, 22.22898 E, $41.1151 \mathrm{~N}, 850 \mathrm{~m}$, phrygana on stony ground) - Rare.

*Geranium pyrenaicum Burm. f. - H scap, ME - Chas. 777 (12/6/2010, 22.18916 E, $41.13403 \mathrm{~N}, 1360 \mathrm{~m}$, forest road in beech stand), Chas. $1376(9 / 6 / 2011,22.1889 \mathrm{E}$, $41.15204 \mathrm{~N}, 1560 \mathrm{~m}$, streambank in beech stand) - Sporadic. In beech stands and forest roads. 
*Geranium reflexum L. - H scap, BI - Chas. 2127 (30/6/2012, 22.22351 E, 41.15024 $\mathrm{N}, 1740 \mathrm{~m}$, tall-herb vegetation above treeline) - Rare. Tall meadows above treeline.

*Geranium robertianum L. - T scap, Ct/Co - Chas. 90 (27/7/2009, 22.1844 E, 41.1335 N, 1190 m), Chas. 126 (6/8/2009, 22.18389 E, 41.15205 N, 1590 m), Chas. 145 (6/8/2009, 22.19144 E, 41.1464 N, 1560 m) - Common. In beech stands.

*Geranium rotundifolium L. - T scap, Pt - Chas. 1278 (28/5/2011, 22.19586 E, $41.11972 \mathrm{~N}, 1120 \mathrm{~m}$, forest road in beech stand) - Rare.

*Geranium sanguineum L. - G rhiz, EA - Chas. 627 (19/5/2010, 22.21658 E, $41.11554 \mathrm{~N}, 920 \mathrm{~m}$, shrubland of thermophilous deciduous and Juniperus oxycedrus) - Common. Thermophilous deciduous and oak forests, mainly on calcareous substrates.

*Geranium subcaulescens DC. - G rhiz, Me - Chas. 148 (6/8/2009, 22.19142 E, $41.14685 \mathrm{~N}, 1560 \mathrm{~m}$, low shrubland at treeline), Chas. $699(2 / 6 / 2010,22.20024 \mathrm{E}$, $41.13807 \mathrm{~N}, 1790 \mathrm{~m}$, subalpine open shrubland) - Common. Grasslands and shrublands above and at treeline. Between 1550 and $2150 \mathrm{~m}$.

*Geranium sylvaticum L. - G rhiz, EA - Chas. 2124 (30/6/2012, 22.22351 E, 41.15024 $\mathrm{N}, 1740 \mathrm{~m}$, tall-herb vegetation above treeline) - Rare. Tall meadows above treeline.

\section{GESNERIACEAE}

Ramonda nathaliae Pančić \& Petrovič - H ros, Bk - Chas. 228 (24/8/2009, 22.21376 E, $41.12306 \mathrm{~N}, 1100 \mathrm{~m}$, rocky roadside in beech stand), Chas. 470 (17/4/2010, $22.22178 \mathrm{E}, 41.11697 \mathrm{~N}, 840 \mathrm{~m}$, rocky slope in ravine with thermophilous deciduous vegetation), Voliotis 1983: 165, MFG2: 261, Schuler 2004: tab. 21 Sporadic. Rocky and stony, usually calcareous and shady, places. 700 - 1900 m.

\section{GLOBULARIACEAE}

Globularia cordifolia L. - C suffr, ME - Chas. 444 (10/4/2010, 22.23019 E, 41.11602 $\mathrm{N}, 890 \mathrm{~m}$, phrygana), Chas. $596(12 / 5 / 2010,22.24717 \mathrm{E}, 41.14303 \mathrm{~N}, 1600 \mathrm{~m}$, open grassland on stony ground), Chas. 1782 (22/4/2012, $22.23195 \mathrm{E}, 41.11542 \mathrm{~N}$, 810 m, phrygana with Juniperus oxycedrus), Voliotis 1983: 165, MFG2: 258 Common. On stony and rocky calcareous places with sparse vegetation. $600-2050$ $\mathrm{m}$.

\section{HYPERICACEAE}

Hypericum barbatum Jacq. - H scap, Eu - Chas. 61 (20/7/2009, 22.21865 E, 41.15367 $\mathrm{N}, 1900 \mathrm{~m}$, subalpine grassland), Chas. 706 (2/6/2010, $22.19803 \mathrm{E}, 41.13173 \mathrm{~N}$, $1610 \mathrm{~m}$, shrubland of Juniperus communis subsp. nana at treeline), Chas. 2283 $(14 / 6 / 2013,22.26927 \mathrm{E}, 41.15481 \mathrm{~N}, 1230 \mathrm{~m}$, forest road in beech stand), MFG1: 603 - Common in grasslands and shrublands at and above treeline, on stony ground. Occasionally descending to $1000 \mathrm{~m}$ in similar habitats.

*Hypericum hirsutum L. - Hscap, ES - Chas. 862 (4/7/2010, 22.17565 E, 41.14408 N, $1370 \mathrm{~m}$, beech stand) - Rare.

*Hypericum maculatum subsp. immaculatum (Murb.) A. Fröhl. - H scap, Bk - Chas. $2220(31 / 7 / 2012,22.17635 \mathrm{E}, 41.15778 \mathrm{~N}, 1860 \mathrm{~m}$, streambank above treeline) Rare. 
*Hypericum montbretii Spach - H scap, EA - Chas. 169 (13/8/2009, 22.18554 E, $41.12244 \mathrm{~N}, 950 \mathrm{~m}$, grassy opening in beech stand), Chas. 1501 (2/7/2011, $22.27995 \mathrm{E}, 41.14713 \mathrm{~N}, 920 \mathrm{~m}$, thermophilous deciduous stand), Chas. 2429 (19/6/2014, 22.27387 E, 41.1292 N, 710 m, oak forest) - Sporadic. Oak forests and thermophilous deciduous stands, on stony ground.

Hypericum olympicum L. - H caesp, BA - Chas. 62 (20/7/2009, 22.21969 E, 41.15477 $\mathrm{N}, 1940 \mathrm{~m}$, subalpine sparse grassland), Chas. $122(6 / 8 / 2009,22.18389 \mathrm{E}$, $41.15205 \mathrm{~N}, 1600 \mathrm{~m}$, forest road in beech stand at treeline), Chas. 839 (26/6/2010, 22.22222 E, 41.12734 N, $1360 \mathrm{~m}$, open shrubland of Juniperus oxycedrus and thermophilous deciduous on rocky slope), MFG1: 597 - Common. At stony and rocky places with sparse vegetation and screes. $800-2100 \mathrm{~m}$.

*Hypericum perforatum L. - H scap, Pt - Chas. 34 (11/7/2009, 22.18 E, 41.12893 N, $1000 \mathrm{~m}$, forest road in beech stand), Chas. 63 (20/7/2009, $22.22073 \mathrm{E}, 41.14704 \mathrm{~N}$, $1780 \mathrm{~m}$, open subalpine shrubland), Chas. 105 (27/7/2009, $22.18653 \mathrm{E}, 41.12975$ $\mathrm{N}, 1190 \mathrm{~m}$, forest road in beech stand), Chas. $2122(30 / 6 / 2012,22.1931 \mathrm{E}$, $41.11625 \mathrm{~N}, 950 \mathrm{~m}$, forest road in beech stand) - Common in forest roads of beech and oak forests. Sporadic in open shrublands and grasslands on stony ground. 600 $1900 \mathrm{~m}$.

Hypericum rumeliacum Boiss. subsp. rumeliacum - H scap, Bk - Chas. 1350 (9/6/2011, 22.21705 E, 41.11149 N, 720 m, forest road in shrubland of Juniperus oxycedrus and thermophilous deciduous), Chas. 1977 (4/6/2012, 22.23859 E, $41.11201 \mathrm{~N}, 680 \mathrm{~m}$, open shrubland of Juniperus oxycedrus and thermophilous deciduous), Voliotis 1983: 165 - Sporadic. At forest roads, phrygana and open shrublands of lowland areas.

\section{JUGLANDACEAE}

*Juglans regia L. - P scap, ME - Chas. 1745 (4/11/2011, 22.26815 E, 41.12921 N, 760 $\mathrm{m}$, forest road in Quercus frainetto stand) - Rare.

\section{LAMIACEAE}

Acinos alpinus subsp. hungaricus (Simonk.) Soják - H caesp, BA - Chas. 1170 $(11 / 5 / 2011,22.20668 \mathrm{E}, 41.1116 \mathrm{~N}, 830 \mathrm{~m}$, forest road in thermophilous deciduous stand), Chas. 1834 (11/5/2012, 22.21382 E, $41.11207 \mathrm{~N}, 780 \mathrm{~m}$, forest road in shrubland of Juniperus oxycedrus and thermophilous deciduous), Voliotis 1983: 165 (as A. alpines subsp. majoranifolius (Mill.) P. W. Ball) - Common. In open shrublands, phrygana and grasslands, on stony ground between 600 and $1800 \mathrm{~m}$.

*Acinos alpinus subsp. meridionalis (Nyman) P.W. Ball - H caesp, Me - Chas. 915 $(1 / 8 / 2010,22.20752 \mathrm{E}, 41.15963 \mathrm{~N}, 2140 \mathrm{~m}$, subalpine sparse grassland on stony ground), Chas. 2114 (30/6/2012, $22.22554 \mathrm{E}, 41.15432 \mathrm{~N}, 1830 \mathrm{~m}$, subalpine sparse grassland on stony ground), Chas. 2215 (31/7/2012, 22.17663 E, 41.15608 $\mathrm{N}, 1810 \mathrm{~m}$, rocky streambank above treeline), Chas. $2321(22 / 6 / 2013,22.19862 \mathrm{E}$, $41.15832 \mathrm{~N}, 1910 \mathrm{~m}$, schistose scree above treeline), Chas. 2415 (30/7/2013, $22.20573 \mathrm{E}, 41.15978 \mathrm{~N}, 2110 \mathrm{~m}$, subalpine sparse grassland on stony ground) Common. Stony places with sparse vegetation, above treeline. $1750-2180 \mathrm{~m}$.

*Acinos arvensis (Lam.) Dandy - $\mathrm{T}$ scap/H bienn, Me - Chas. 1489 (2/7/2011, 22.27438 E, 41.13714 N, 870 m, forest road in Quercus frainetto stand) - Rare.

Ajuga chamaepitys subsp. chia (Schreb.) Arcang. - H scap, EA - Chas. 399 (9/10/2009, 22.22604 E, $41.11388 \mathrm{~N}, 780 \mathrm{~m}$, phrygana on rocky ground), Chas. 509 (2/5/2010, 
22.22395 E, 41.11187 N, 680 m, dry grassland), Voliotis 1983: 165 - Sporadic. Dry grasslands and phrygana, on stony ground between 600 and $1000 \mathrm{~m}$.

Ajuga genevensis L. - G rhiz, EA - Chas. 700 (2/6/2010, 22.19803 E, 41.13173 N, $1610 \mathrm{~m}$, open Pinus nigra stand on rocky ground at treeline), Chas. 1850 (16/5/2012, 22.20162 E, 41.12251 N, 1190 m, Juniperus oxycedrus - thermophilous deciduous stand within beech stand), Voliotis 1983: 165 - Sporadic. In grasslands, phrygana, shrublands and forest roads, up to $1500 \mathrm{~m}$.

Ajuga reptans L. - H rept, EA - Chas. 483 (25/4/2010, 22.19832 E, 41.10823 N, 770 m, damp grassland), Chas. 1173 (11/5/2011, $22.21522 \mathrm{E}, 41.1393 \mathrm{~N}, 1540 \mathrm{~m}$, ditch by roadside in beech stand), Voliotis 1983: 165 - Sporadic. Damp places. 700 $1700 \mathrm{~m}$.

*Ballota nigra subsp. uncinata (Fiori\&Bég.) Patzak - H scap, Me - Chas. 396 (9/10/2009, 22.22863 E, $41.12356 \mathrm{~N}, 1270 \mathrm{~m}$, grassland within Juniperus oxycedrus - thermophilous deciduous shrubland), Chas. 2160 (6/7/2012, 22.28619 E, $41.13182 \mathrm{~N}, 670 \mathrm{~m}$, road in dry grassland) - Rare.

Betonica alopecuros L. - H scap, Eu - Chas. 1564 (22/7/2011, 22.17674 E, 41.15203 N, $1750 \mathrm{~m}$, grassy opening in low shrubland at treeline), MFG2: 98 (as Stachys alopecuros (L.) Benth.) - Sporadic. In grasslands at and above treeline.

*Betonica scardica Griseb. - H scap, Bk - Chas. 871 (4/7/2010, 22.17565 E, 41.14408 $\mathrm{N}, 1370 \mathrm{~m}$, beech stand) - Rare.

*Calamintha grandiflora (L.) Moench - H scap, ME - Chas. 94 (27/7/2009, 22.1844 E, $41.1335 \mathrm{~N}, 1190 \mathrm{~m})$, Chas. 130 (6/8/2009, 22.18389 E, 41.15205 N, $1590 \mathrm{~m})$, Chas. 1558 (17/7/2011, 22.19064 E, 41.13189 N, 1340 m) - Common. Beech stands, from 1000 up to $1800 \mathrm{~m}$.

*Calamintha menthifolia Host - H scap, ME - Chas. 409 (17/10/2009, 22.24754 E, $41.13367 \mathrm{~N}, 1140 \mathrm{~m}$, forest road in mixed beech - oak stand), Chas. 1682 (10/9/2011, 22.26678 E, 41.15396 N, 1190 m, beech stand), Chas. 2180 (11/7/2012, 22.18209 E, 41.12662 N, 1020 m, beech stand) - Sporadic. Beech stands, oak forests and forest roads.

*Calamintha nepeta (L.) Savi - H scap, ME - Chas. 185 (13/8/2009, 22.18661 E, $41.12282 \mathrm{~N}, 1000 \mathrm{~m}$, forest road in beech stand), Chas. $293(10 / 9 / 2009,22.26185$ E, $41.12866 \mathrm{~N}, 790 \mathrm{~m}$, forest road in Quercus frainetto stand), Chas. 1640 $(23 / 8 / 2011,22.19823 \mathrm{E}, 41.12039 \mathrm{~N}, 1110 \mathrm{~m}$, forest road in mixed beech thermophilous deciduous stand) - Sporadic. Thermophilous deciduous shrublands and forests and in forest roads of lowland areas.

*Clinopodium vulgare subsp. orientale Bothmer - H scap, Me - Chas. 12 (11/7/2009, 22.18 E, 41.12893 N, $1000 \mathrm{~m}$, beech stand), Chas. 2121 (30/6/2012, $22.1931 \mathrm{E}$, $41.11625 \mathrm{~N}, 970 \mathrm{~m}$, forest road in thermophilous deciduous stand) - Common. Thermophilous deciduous stands, oak forests and thermophilous beech forests.

*Clinopodium vulgare L. subsp. vulgare - H scap, ES - Chas. 1488 (2/7/2011, 22.27426 E, 41.13714 N, $880 \mathrm{~m}$, forest road in mixed oak - beech stand), Chas. $2102(30 / 6 / 2012,22.22261 \mathrm{E}, 41.14806 \mathrm{~N}, 1790 \mathrm{~m}$, subalpine grassland), Chas. 2287 (14/6/2013, 22.2647 E, 41.16059 N, 1550 m, Pteridium thicket) - Sporadic. Subalpine grasslands and Pteridium thickets and grasslands at treeline.

?Galeobdolon luteum Huds. - Voliotis 1983: 165, Schuler 2004: map 52.

Galeobdolon montanum (Pers.) Rchb. - H scap, Eu - Chas. 663 (24/5/2010, 22.25993 E, 41.15629 N, $1400 \mathrm{~m}$, beech stand), Chas. 1117 (26/4/2011, 22.19177 E, 
41.11263 N, 720 m, Alnus stand), Schuler 2004: map 53 (as Lamium montanum (Pers.) Hoffm. ex Kabath) - Common. In beech stands.

*Galeopsis bifida Boenn. - T scap, ES - Chas. 2221 (31/7/2012, 22.1794 E, 41.15063 N, 1680 m), Chas. 2237 (9/8/2012, 22.21423 E, 41.13622 N, 1470 m) - Sporadic. Beech stands on mesic or damp ground.

Lamium amplexicaule L. - T scap, Pt - Chas. 452 (10/4/2010, 22.23015 E, 41.11602 N, 890 m, phrygana), Voliotis 1983: 165 - Sporadic. Dry grasslands and phrygana of lowland areas.

*Lamium bifidum subsp. balcanicum Velen. - T scap, Bk - Chas. 1859 (16/5/2012, 22.21739 E, 41.14293 N, $1630 \mathrm{~m}$, grassland at treeline) - Rare.

Lamium garganicum L. subsp. garganicum - H scap, Me - Chas. 431 (15/11/2009, 22.2212 E, $41.11705 \mathrm{~N}, 840 \mathrm{~m}$, rocky opening in thermophilous deciduous stand), Chas. $467(17 / 4 / 2010,22.22058 \mathrm{E}, 41.11731 \mathrm{~N}, 850 \mathrm{~m}$, grassland on stony ground within thermophilous deciduous stand), Chas. 1017 (8/4/2011, 22.24168 E, $41.11214 \mathrm{~N}, 680 \mathrm{~m}$, thermophilous deciduous stand), Voliotis 1983: 165 (as $L$. garganicum subsp. laevigatum Arcang.) - Sporadic. Stony places of lowland areas.

Lamium maculatum (L.) L. - H scap, EA - Chas. 254 (28/8/2009, 22.20974 E, $41.13939 \mathrm{~N}, 1700 \mathrm{~m}$, forest road in beech stand), Chas. $560(8 / 5 / 2010,22.22256 \mathrm{E}$, 41.15013 N, 1750 m, tall-herb subalpine meadow), Voliotis 1983: 165 - Sporadic. Beech forests and above treeline tall-herb meadows, on mesic ground. $800-2000$ $\mathrm{m}$.

*Lamium purpureum L. - T scap, EA - Chas. 998 (28/3/2011, 22.19086 E, 41.11541 $\mathrm{N}, 860 \mathrm{~m}$, opening in thermophilous deciduous stand) - Rare. Grasslands and roads, up to $900 \mathrm{~m}$.

*Lycopus europaeus L. - H scap, Pt - Chas. 230 (24/8/2009, 22.21376 E, 41.12306 N, $1100 \mathrm{~m}$, damp place at forest road in beech stand) - Rare.

*Marrubium peregrinum L. - H scap, Eu - Chas. 316 (10/9/2009, 22.26703 E, $41.12907 \mathrm{~N}, 750 \mathrm{~m}$, dry grassland) - Rare. In dry grasslands and forest roads, up to $800 \mathrm{~m}$.

*Melissa officinalis subsp. altissima (Sm.) Arcang. - H scap, Me - Chas. 2426 (9/6/2014, 22.22503 E, 41.11153 N, 660 m) - Rare. In Platanus stands.

*Mentha longifolia (L.) L. - H scap, Pt - Chas. 6 (11/7/2009, 22.18055 E, 41.13065 N, $1050 \mathrm{~m}$, ditch by roadside in beech stand) - Sporadic. Ditches by roadside and other damp places, up to $1400 \mathrm{~m}$.

* Mentha spicata subsp. condensata (Briq.) Greuter \& Burdet - Hscap, Me - Chas. 1680 (10/9/2011, 22.26224 E, 41.14092 N, 1120 m) - Sporadic. Ditches by roadside and other damp places, up to $1400 \mathrm{~m}$.

Micromeria cristata (Hampe) Griseb. - C suffr, BA - Chas. 193 (24/8/2009, 22.21624 E, $41.12382 \mathrm{~N}, 1070 \mathrm{~m}$, opening on rocky ground in beech stand), Chas. 363 $(15 / 9 / 2009,22.25927 \mathrm{E}, 41.16312 \mathrm{~N}, 1760 \mathrm{~m}$, rocky place above treeline), MFG2: 133 - Sporadic. Rocky open calcareous places, between 1000 and $2050 \mathrm{~m}$.

Micromeria juliana (L.) Benth. ex Rchb. - C suffr, Me - Chas. 971 (31/10/2010, 22.23146 E, 41.11577 N, 840 m), Chas. 1346 (9/6/2011, 22.21799 E, 41.11205 N, $720 \mathrm{~m}$ ), Voliotis 1983: 165 - Sporadic. At stony and rocky places with phrygana. Between 600 and $1300 \mathrm{~m}$.

*Nepeta nuda L. subsp. nuda - H scap, EA - Chas. 140 (6/8/2009, 22.19142 E, $41.14685 \mathrm{~N}, 1560 \mathrm{~m}$, grassy opening in beech stand), Chas. 2024 (13/6/2012, 22.22851 E, $41.12365 \mathrm{~N}, 1280 \mathrm{~m}$, grassland within shrubland of thermophilous 
deciduous and Juniperus oxycedrus) - Sporadic. Mesic grasslands, between 1000 and $1800 \mathrm{~m}$.

*Origanum vulgare L. subsp. vulgare - H scap/G rhiz, EA - Chas. 160 (13/8/2009, 22.18554 E, 41.12244 N, $950 \mathrm{~m}$, grassy opening in beech stand) - Common in oak forests. Sporadic in thermophilous deciduous stands and thermophilous beech stands.

*Prunella laciniata (L.) L. - H scap, Me - Chas. 1517 (7/7/2011, 22.19694 E, 41.1065 N, 730 m, open Juniperus oxycedrus - thermophilous deciduous shrubland), Chas. 2416 (30/7/2013, $22.19515 \mathrm{E}, 41.1588 \mathrm{~N}, 1900 \mathrm{~m}$, streambank above treeline) Sporadic. Stony places with sparse shrubs, phrygana or herbaceous vegetation, between 650 and $1900 \mathrm{~m}$.

Prunella vulgaris L. - H scap, EA - Chas. 15 (11/7/2009, 22.18 E, 41.12893 N, 1000 m, mesic beech stand), Chas. 2417 (30/7/2013, 22.19515 E, 41.1588 N, $1900 \mathrm{~m}$, streambank above treeline), Schuler 2004: tab. 20 - Sporadic in mesic beech forests. Rare in mesic to damp grasslands at and above treeline.

*Salvia amplexicaulis Lam. - H scap, BA - Chas. 1440 (22/6/2011, 22.22068 E, $41.10921 \mathrm{~N}, 640 \mathrm{~m}$, dry grassland) - Rare.

*Salvia argentea L. - H scap, Me - Chas. 1898 (24/5/2012, 22.18481 E, 41.12324 N, $660 \mathrm{~m}$, phrygana) - Rare. In phrygana and open shrublands of Juniperus oxycedrus, up to $800 \mathrm{~m}$.

*Salvia glutinosa L. - H scap, EA - Chas. 164 (13/8/2009, 22.18481 E, 41.12324 N, $950 \mathrm{~m}$, streambank in beech stand) - Sporadic. Beech forests on mesic to damp ground, between 950 and $1600 \mathrm{~m}$.

*Salvia ringens Sm. - H scap, Eu - Chas. 419 (17/10/2009, 22.23613 E, 41.13268 N, $1350 \mathrm{~m}$, open thermophilous deciduous stand on stony ground), Chas. 770 $(12 / 6 / 2010,22.21467 \mathrm{E}, 41.11119 \mathrm{~N}, 740 \mathrm{~m}$, rocky roadside in shrubland of Juniperus oxycedrus and thermophilous deciduous) - Sporadic. Stony or rocky ground with sparse vegetation, between 650 and $1450 \mathrm{~m}$.

*Salvia sclarea L. - H bienn, MS - Chas. 1516 (7/7/2011, 22.20231 E, 41.10613 N, 700 $\mathrm{m}$, open shrubland of Juniperus oxycedrus) - Sporadic. In shrublands on stony ground, between 600 and $850 \mathrm{~m}$.

*Salvia viridis L. - Tscap, Me - Chas. 1965 (4/6/2012, 22.25459 E, 41.10603 N, 600 m, dry grassland) - Rare.

*Satureja montana subsp. macedonica (Formánek) Baden - C suffr, Gr - Chas. 1692 $(13 / 9 / 2011,22.22157 \mathrm{E}, 41.11174 \mathrm{~N}, 690 \mathrm{~m}$, rocky roadside in shrubland of thermophilous deciduous and Juniperus oxycedrus), Chas. 1718 (24/9/2011, 22.23603 E, 41.12773 N, 1200 m, sparse grassland on stony ground) - Sporadic. Stony and rocky ground with sparse vegetation, between 600 and $1300 \mathrm{~m}$.

*Satureja montana L. subsp. montana - C suffr, Me - Chas. 204 (24/8/2009, 22.21624 E, $41.12382 \mathrm{~N}, 1070 \mathrm{~m}$, rocky opening in beech stand), Chas. 408 (9/10/2009, $22.22865 \mathrm{E}, 41.12284 \mathrm{~N}, 1250 \mathrm{~m}$, stony grassland), Chas. 1733 (4/10/2011, 22.22709 E, 41.12795 N, $1480 \mathrm{~m}$, stony grassland) - Sporadic. Stony or rocky ground with sparse vegetation, between 600 and $1550 \mathrm{~m}$.

*Scutellaria columnae All. - H scap, Me - Chas. 1505 (2/7/2011, 22.27636 E, 41.14955 N, 1030 m, Quercus frainetto stand) - Sporadic. In oak stands.

*Sideritis montana L. subsp. montana - T scap, MS - Chas. 629 (19/5/2010, 22.21658 E, $41.11554 \mathrm{~N}, 920 \mathrm{~m}$, open shrubland on stony ground) - Sporadic. Open shrublands, phrygana and dry grasslands of lowland areas. 
*Sideritis montana subsp. remota (d'Urv.) P. W. Ball - T scap, MS - Chas. 1915 (24/5/2012, 22.22723 E, 41.11336 N, 750 m, phrygana) - Sporadic. Open shrublands, phrygana and dry grasslands of lowland areas.

Sideritis scardica Griseb. - H scap, Bk - Chas. 336 (15/9/2009, 22.26011 E, 41.16286 N, $1760 \mathrm{~m})$, MFG1: 89 - Sporadic. Subalpine grasslands, on stony calcareous ground.

*Stachys alpina L. - H scap, Eu - Chas. 2085 (25/6/2012, 22.21404 E, 41.13901 N, $1540 \mathrm{~m}$, forest road in beech stand), Chas. 2327 (26/6/2013, 22.23103 E, 41.15855 N, 1690 m, streambank with tall-herb vegetation above treeline) - Sporadic. Above treeline grasslands and openings of beech stands, above $1500 \mathrm{~m}$.

Stachys horvaticii Micevski - H scap, Bk - Chas. 329 (15/9/2009, 22.26011 E, $41.16286 \mathrm{~N}, 1760 \mathrm{~m}$, subalpine grassland on stony ground), Chas. 610 (19/5/2010, $22.2149 \mathrm{E}, 41.11623 \mathrm{~N}, 1000 \mathrm{~m}$, rocky place with sparse herb vegetation), Voliotis 1983: 165, MFG2: 107 - Common. In grasslands on stony or rocky calcareous ground. Between 1000 and $2050 \mathrm{~m}$.

*Stachys plumosa Griseb. - H scap, Bk - Chas. 194 (24/8/2009, 22.21376 E, 41.12306 $\mathrm{N}, 1120 \mathrm{~m}$, roadside in mixed beech - thermophilous deciduous stand), Chas. 208 $(24 / 8 / 2009,22.21376 \mathrm{E}, 41.12306 \mathrm{~N}, 1120 \mathrm{~m}$, roadside in mixed beech thermophilous deciduous stand), Chas. 1690 (10/9/2011, 22.26036 E, $41.16242 \mathrm{~N}$, $1740 \mathrm{~m}$, subalpine grassland) - Sporadic. Thermophilous deciduous stands, roads and grasslands, between 1000 and $1750 \mathrm{~m}$.

*Stachys sylvatica L. - H scap, ES - Chas. 86 (27/7/2009, 22.1844 E, 41.1335 N, 1190 m), Chas. 864 (4/7/2010, 22.17565 E, 41.14408 N, 1370 m) - Sporadic. In mesic beech stands.

Stachys tymphaea Hausskn. - H scap, BI - Chas. 142 (6/8/2009, 22.19142 E, 41.14685 N, 1560 m), Chas. 1636 (23/8/2011, 22.21537 E, 41.13822 N, 1510 m), Parent 2005: 215 - Sporadic. At grassy openings in beech forests, at treeline and above.

*Teucrium capitatum L. - C suffr, Me - Chas. 8 (11/7/2009, 22.21126 E, 41.10969 N, $730 \mathrm{~m}$, dry grassland on stony ground), Chas. 893 (24/7/2010, 22.2568 E, 41.14849 $\mathrm{N}, 1560 \mathrm{~m}$, grassland on stony ground within beech stand) - Common. Dry grasslands and phrygana at low and mid-altitudes.

Teucrium chamaedrys L. subsp. chamaedrys - C suffr, Me - Chas. 414 (17/10/2009, $22.2359 \mathrm{E}, 41.1325 \mathrm{~N}, 1340 \mathrm{~m}$, open thermophilous deciduous stand on stony ground), Chas. $805(26 / 6 / 2010,22.22203 \mathrm{E}, 41.1167 \mathrm{~N}, 840 \mathrm{~m}$, thermophilous deciduous stand), Chas. 1627 (18/8/2011, 22.23031 E, $41.14548 \mathrm{~N}, 1950 \mathrm{~m}$, subalpine grassland), Voliotis 1983: 165 - Common in thermophilous deciduous stands, oak forests, grasslands and phrygana. Between 600 and $2000 \mathrm{~m}$, mainly on calcareous substrates.

*Teucrium montanum subsp. helianthemoides (Adamovic) Baden - C suffr, Gr Chas. $838(26 / 6 / 2010,22.22292 \mathrm{E}, 41.12789 \mathrm{~N}, 1400 \mathrm{~m}$, rocky slope with open thermophilous deciduous shrubland), Chas. 1626 (18/8/2011, 22.23031 E, 41.14557 N, $1950 \mathrm{~m}$, subalpine sparse grassland on stony ground) - Sporadic. Sparse grasslands on stony or rocky calcareous ground.1400 - $2050 \mathrm{~m}$.

* Thymus boissieri Halácsy - C suffr, Bk - Chas. 668 (24/5/2010, 22.24497 E, 41.15867 $\mathrm{N}, 1820 \mathrm{~m}$, subalpine sparse grassland on stony ground), Chas. 842 (26/6/2010, $22.22398 \mathrm{E}, 41.12835 \mathrm{~N}, 1440 \mathrm{~m}$, rocky slope with open thermophilous deciduous shrubland), Chas. 2290 (14/6/2013, 22.26275 E, 41.16245 N, 1700 m, subalpine 
sparse grassland on stony ground) - Common. Sparse grasslands on stony or rocky calcareous ground. $1400-2050 \mathrm{~m}$.

*Thymus longicaulis subsp. chaubardii (Rchb. f.) Jalas - C suffr, BA - Chas. 642 $(19 / 5 / 2010,22.21624$ E, $41.11932 \mathrm{~N}, 1110 \mathrm{~m}$, rocky slope with open thermophilous deciduous shrubland), Chas. 1198 (20/5/2011, 22.22998 E, 41.11773 N, 990 m, grassland on stony ground) - Sporadic. Grasslands. $900-2100 \mathrm{~m}$.

Thymus praecox subsp. jankae (Č́elak.) Jalas - C suffr, BA - Chas. 2218 (31/7/2012, $22.17663 \mathrm{E}, 41.15608 \mathrm{~N}, 1810 \mathrm{~m}$, rocky streambank above treeline), Chas. 2398 (25/7/2013, 22.20978 E, 41.15534 N, 2060 m, subalpine sparse grassland on stony ground), Voliotis 1983: 165 (as Th. praecox subsp. skorpilii (Velen.) Jalas), MFG2: 163, Schuler 2004: tab. 21 - Common. Subalpine stony ground, usually with sparse vegetation. $1600-2180 \mathrm{~m}$.

Thymus sibthorpii Benth. - C suffr, BA - Chas. 687 (2/6/2010, 22.215 E, 41.11254 N, $780 \mathrm{~m}$, roadside in thermophilous deciduous stand), Chas. 814 (26/6/2010, $22.22203 \mathrm{E}, 41.1167 \mathrm{~N}, 840 \mathrm{~m}$, grassy opening in thermophilous deciduous stand), Chas. $1412(16 / 6 / 2011,22.22585$ E, $41.11649 \mathrm{~N}, 880 \mathrm{~m}$, open shrubland of Juniperus oxycedrus and thermophilous deciduous), Voliotis 1983: 165 - Common. Grasslands, phrygana and shrublands. $600-2150 \mathrm{~m}$.

*Thymus striatus Vahl - C suffr, BI - Chas. 831 (26/6/2010, 22.22452 E, 41.11269 N, 700 m), Chas. 1413 (16/6/2011, 22.22661 E, 41.11479 N, 850 m) - Rare. On stony or rocky calcareous ground with phrygana.

*Thymus thracicus Velen. - C suffr, Me - Chas. 1131 (1/5/2011, 22.22197 E, 41.11445 $\mathrm{N}, 810 \mathrm{~m}$, phrygana), Chas. 1887 (24/5/2012, $22.20876 \mathrm{E}, 41.11398 \mathrm{~N}, 880 \mathrm{~m}$, open thermophilous deciduous stand with Juniperus oxycedrus) - Sporadic. Grasslands, phrygana and open thermophilous deciduous shrublands on stony or rocky calcareous ground. $700-2050 \mathrm{~m}$.

\section{LENTIBULARIACEAE}

Pinguicula balcanica Casper subsp. balcanica - H ros, Bk - Chas. 754 (12/6/2010, 22.20086 E, 41.15907 N, 1930 m, streambank above treeline), Parent 2005: 218 Sporadic. In damp places, mainly eroded, above treeline.

\section{LINACEAE}

*Linum austriacum L. subsp. austriacum - C suffr, EA - Chas. 1203 (20/5/2011, $22.22834 \mathrm{E}, 41.12122 \mathrm{~N}, 1150 \mathrm{~m}$, grassland within shrubland of Juniperus oxycedrus and thermophilous deciduous), Chas. 2015 (13/6/2012, 22.23007 E, $41.11827 \mathrm{~N}, 1010 \mathrm{~m}$, grassland within shrubland of Juniperus oxycedrus and thermophilous deciduous) - Sporadic. Grasslands at low and mid-altitudes.

*Linum austriacum subsp. collinum (Boiss.) Nyman - C suffr, Me - Chas. 1899 $(24 / 5 / 2012,22.2323$ E, $41.11137 \mathrm{~N}, 660 \mathrm{~m}$, forest road in open Juniperus oxycedrus - thermophilous deciduous shrubland), Chas. 1900 (24/5/2012, 22.23326 E, 41.11111 N, 650 m, phrygana) - Rare.

*Linum bienne Miller - T scap, Me - Chas. 2438 (28/6/2014, 22.20208 E, 41.11 N, 830 $\mathrm{m}$, dry grassland) - Rare.

Linum capitatum Schultes subsp. capitatum - C suffr, Bk - Chas. 742 (12/6/2010, 22.17429 E, 41.15442 N, 1890 m), Chas. 2455 (14/7/2014, 22.23271 E, 41.1447 N, $2030 \mathrm{~m})$, Chasapis et al. 2019 - Sporadic. In shrublands at and above treeline. 
Linum catharticum L. - T scap/H scap, Me - Chas. 1381 (9/6/2011, 22.20728 E, $41.11143 \mathrm{~N}, 820 \mathrm{~m}$, Pteridium thicket), Chas. $1620(18 / 8 / 2011,22.21823 \mathrm{E}$, $41.14691 \mathrm{~N}, 1790 \mathrm{~m}$, ditch by roadside above treeline), Schuler 2004: tab. 20 Common. Damp open places, up to $1950 \mathrm{~m}$.

*Linum flavum subsp. albanicum (Janch.) Hartvig - H scap, Bk - Chas. 813 $(26 / 6 / 2010,22.22359 \mathrm{E}, 41.11637 \mathrm{~N}, 790 \mathrm{~m}$, rocky opening in thermophilous deciduous stand), Chas. 932 (8/8/2010, $22.22798 \mathrm{E}, 41.1258 \mathrm{~N}, 1360 \mathrm{~m}$, open thermophilous deciduous shrubland), Chas. 1414 (16/6/2011, 22.2262 E, 41.1165 $\mathrm{N}, 880 \mathrm{~m}$, open thermophilous deciduous shrubland), Chas. 2010 (13/6/2012, 22.22629 E, $41.11758 \mathrm{~N}, 940 \mathrm{~m}$, open thermophilous deciduous shrubland), Chas. 2186 (15/7/2012, 22.24427 E, 41.15353 N, $1760 \mathrm{~m}$, subalpine shrubland), Chas. 2303 (18/6/2013, 22.23678 E, 41.13963 N, 1640 m, shrubland of Juniperus communis subsp. alpina) - Common. Shrublands and grasslands on stony ground, between 700 and $2000 \mathrm{~m}$.

Linum hirsutum subsp. spathulatum (Halácsy \& Bald.) Hayek - H scap, Bk - Chas. $714(2 / 6 / 2010,22.215 \mathrm{E}, 41.11254 \mathrm{~N}, 780 \mathrm{~m}$, forest road in thermophilous deciduous stand), Chas. 1980 (4/6/2012, 22.25165 E, $41.11815 \mathrm{~N}, 730 \mathrm{~m}$, dry grassland), Chas. 2346 (1/7/2013, $22.22925 \mathrm{E}, 41.13591 \mathrm{~N}, 1620 \mathrm{~m}$, sparse grassland on stony ground at treeline), Voliotis 1983: 166 (as L. spathulatum (Halácsy \& Bald.) Halácsy) - Sporadic. Forest roads, grasslands and phrygana, on stony ground at low and mid-altitudes.

*Linum hologynum Reichenb. - H scap, Bk - Chas. 1382 (9/6/2011, 22.20728 E, $41.11143 \mathrm{~N}, 820 \mathrm{~m}$, Pteridium thicket), Chas. 1584 (22/7/2011, $22.17662 \mathrm{E}$, $41.15229 \mathrm{~N}, 1770 \mathrm{~m}$, grassy opening in subalpine shrubland) - Sporadic. In dry grasslands of lowland areas, occasionally abundant.

*Linum strictum subsp. spicatum Pers. (Nyman) - T scap, Me - obs. (28/6/2014, 22.23941 E, 41.11256 N, 700 m, dry grassland) - Rare.

Linum tenuifolium L. - H scap, EA - Chas. 630 (19/5/2010, 22.21658 E, 41.11554 N, $940 \mathrm{~m})$, Chas. 1202 (20/5/2011, $22.22928 \mathrm{E}, 41.11736 \mathrm{~N}, 990 \mathrm{~m})$, Voliotis 1983: 166, MFG1: 564 - Sporadic. In phrygana on stony ground of lowland areas.

*Linum trigynum L. - T scap, Me - Chas. 1443 (22/6/2011, 22.22068 E, $41.10921 \mathrm{~N}$, $640 \mathrm{~m})$, Chas. $2440(28 / 6 / 2014,22.20208$ E, $41.11 \mathrm{~N}, 830 \mathrm{~m})$ - Sporadic. In dry grasslands of lowland areas.

\section{LYTHRACEAE}

*Lythrum salicaria L. - H scap, EA/[Co] - Chas. 2 (11/7/2009, 22.20585 E, 41.11168 $\mathrm{N}, 830 \mathrm{~m}$, ditch by roadside in thermophilous deciduous stand) - Rare.

\section{MALVACEAE}

*Malva sylvestris L. - H scap/T scap, EA - Chas. 401 (9/10/2009, 22.22873 E, $41.11987 \mathrm{~N}, 1100 \mathrm{~m}$, grassy opening in thermophilous deciduous and Juniperus oxycedrus shrubland) - Rare.

Malva thuringiaca subsp. ambigua (DC.) Valdés - H scap, BI - Chas. 296 (10/9/2009, 22.25709 E, 41.1285 N, $880 \mathrm{~m}$, forest road in Quercus frainetto stand), Strid \& Papanicolaou 1981: 72 (as Lavatera thuringiaca L.) - Sporadic. In forest roads of lowland areas.

\section{MORACEAE}


*Ficus carica L. - P caesp, MS - Chas. 1272 (28/5/2011, 22.22133 E, 41.11156 N, 690 $\mathrm{m}$, road in thermophilous deciduous and Juniperus oxycedrus shrubland) - Rare.

\section{OLEACEAE}

*Fraxinus ornus L. - P scap/P caesp, ME - Chas. 95 (27/7/2009, 22.1844 E, 41.1335 N, 1190 m, beech stand), Chas. 844 (26/6/2010, 22.21917 E, 41.1254 N, $1220 \mathrm{~m}$, open thermophilous deciduous shrubland on rocky slope) - Common. In mixture with other thermophilous deciduous. Rare in beech stands at the lower parts of the beech forest zone.

*Jasminum fruticans L. - P caesp, EA - Chas. 613 (19/5/2010, 22.2155 E, 41.11597 N, $950 \mathrm{~m}$, thermophilous deciduous stand on stony ground) - Rare.

*Ligustrum vulgare L. - P caesp, ME - Chas. 950 (14/8/2010, 22.19389 E, 41.10483 N, $630 \mathrm{~m}$, thermophilous deciduous shrubland), Chas. 1416 (16/6/2011, 22.22659 E, $41.11551 \mathrm{~N}, 880 \mathrm{~m}$, thermophilous deciduous and Juniperus oxycedrus shrubland) - Common. In thermophilous deciduous stands and oak forests.

*Phillyrea latifolia L. - P caesp, Me - Chas. 1752 (18/3/2012, 22.23847 E, 41.112 N, 680 m, open Juniperus oxycedrus and thermophilous deciduous shrubland) - Rare.

\section{ONAGRACEAE}

*Circaea lutetiana L. - H scap, EA - Chas. 1723 (28/9/2011, 22.19177 E, 41.11272 N, 720 m, swampy place with Alnus glutinosa) - Rare.

*Epilobium alsinifolium Vill. - H scap, AA - Chas. 1573 (22/7/2011, 22.17448 E, 41.15613 N, $1900 \mathrm{~m})$, Chas. 2403 (25/7/2013, 22.21823 E, $41.14691 \mathrm{~N}, 1790 \mathrm{~m})$ Sporadic. In springs and streams above treeline.

*Epilobium anagallidifolium Lam. - H scap, AA - Chas. 2213 (31/7/2012, 22.17664 E, $41.1559 \mathrm{~N}, 1800 \mathrm{~m}$, above treeline stream) - Rare.

Epilobium angustifolium L. - H scap, Ct - Chas. 874 (4/7/2010, 22.18017 E, 41.13154 $\mathrm{N}, 1060 \mathrm{~m}$, roadside in beech stand), Chas. 1601 (13/8/2011, 22.21505 E, 41.15893 N, 2050 m, streambank above treeline), Schuler 2004: map 40 - Common at roadsides in beech stands. Rare at streambanks above treeline. $1000-2050 \mathrm{~m}$.

*Epilobium dodonaei Vill. - H scap, Me - Chas. 242 (28/8/2009, 22.20444 E, 41.124 $\mathrm{N}, 1200 \mathrm{~m}$, stony roadside in beech stand) - Rare.

*Epilobium hirsutum L. - H scap, EA - Chas. 226 (24/8/2009, 22.21376 E, 41.12306 $\mathrm{N}, 1100 \mathrm{~m}$, ditch by roadside in beech stand) - Sporadic. Ditches by roadsides and streambanks in the forest zone.

*Epilobium Ianceolatum Sebastiani \& Mauri - H scap, EA - Chas. 1553 (17/7/2011, 22.18979 E, 41.12836 N, 1230 m, ditch by roadside in beech stand) - Rare. Ditches by roadsides and streambanks in beech stands.

*Epilobium montanum L. - H scap, EA - Chas. 153 (6/8/2009, 22.19132 E, 41.14649 N, $1560 \mathrm{~m}$, beech stand), Chas. 271 (28/8/2009, $22.20973 \mathrm{E}, 41.13966 \mathrm{~N}, 1760 \mathrm{~m}$, ditch by roadside in beech stand) - Common. In mesic beech stands.

*Epilobium palustre L. - H scap, Bo - Chas. 2390 (20/7/2013, 22.2391 E, 41.15525 N, $1750 \mathrm{~m}$, above treeline fen) - Rare.

*Epilobium parviflorum Schreber - H scap, Pt - Chas. 393 (27/9/2009, 22.20742 E, $41.1372 \mathrm{~N}, 1680 \mathrm{~m}$, ditch by roadside in beech stand), Chas. $1596(13 / 8 / 2011$, 22.22706 E, $41.1112 \mathrm{~N}, 660 \mathrm{~m}$, streambank in grassland) - Sporadic. At open damp places, between 650 and $1700 \mathrm{~m}$. 
*Epilobium roseum Schreber subsp. roseum - H scap, EA - Chas. 1707 (18/9/2011, 22.17949 E, 41.13874 N, 1180 m, stream in beech stand) - Rare.

\section{OROBANCHACEAE}

Bellardia latifolia (L.) Cuatrec. - T scap, MS - Chas. 490 (25/4/2010, 22.19838 E, $41.10616 \mathrm{~N}, 700 \mathrm{~m}$, grassy opening in shrubland of Juniperus oxycedrus and thermophilous deciduous), Voliotis 1983: 168 (as Parentucellia latifolia (L.) Caruel) - Common. In dry grasslands and forest openings of lowland areas.

Euphrasia liburnica Wettst. - T scap, BI - Chas. 265 (28/8/2009, 22.21319 E, $41.14404 \mathrm{~N}, 1760 \mathrm{~m}$, opening in subalpine shrubland), Chas. 1657 (23/8/2011, 22.21864 E, $41.16718 \mathrm{~N}, 2040 \mathrm{~m}$, subalpine sparse grassland on stony ground), Chas. 2401 (25/7/2013, 22.22149 E, $41.15876 \mathrm{~N}, 1850 \mathrm{~m}$, above treeline fen), Schuler 2004: tab. 21 - Common. Subalpine grasslands, mainly on stony ground.

Euphrasia pectinata Ten. - T scap, ES - Chas. 21 (11/7/2009, 22.18 E, 41.12893 N, $1000 \mathrm{~m}$, forest road in beech stand), Voliotis 1983: 168 - Rare.

*Euphrasia salisburgensis Funck - T scap, AA - Chas. 206 (24/8/2009, 22.21376 E, $41.12306 \mathrm{~N}, 1100 \mathrm{~m}$, damp place at forest road in beech stand), Chas. 1666 (31/8/2011, 22.18757 E, $41.14418 \mathrm{~N}, 1440 \mathrm{~m}$, damp place at forest road in beech stand) - Sporadic. Damp places in beech stands and above treeline.

*Lathraea squamaria L. - H caesp, EA - Chas. 987 (28/3/2011, 22.19177 E, 41.11272 N, 720 m, swampy place with alders), Chas. 1093 (26/4/2011, 22.19177 E, 41.11263 N, 720 m, swampy place with alders) - Rare.

*Macrosyringion glutinosum (M. Bieb.) Rothm. - T scap, BA - Chas. 349 (15/9/2009, $22.25988 \mathrm{E}, 41.16286 \mathrm{~N}, 1750 \mathrm{~m}$, subalpine grassland on stony ground), Chas. $1689(10 / 9 / 2011,22.26475 \mathrm{E}, 41.16347 \mathrm{~N}, 1690 \mathrm{~m}$, sparse grassland on stony ground) - Sporadic. Stony and rocky calcareous places with sparse vegetation, at and above treeline.

*Odontites vulgaris Moench - H scap, EA - Chas. 1659 (31/8/2011, 22.2049 E, 41.11167 N, $840 \mathrm{~m}$, forest road in Quercus frainetto stand) - Rare.

*Orobanche alba Willd. - T par, EA - Chas. 220 (24/8/2009, 22.21415 E, 41.12172 N, $1100 \mathrm{~m}$, grassy opening in thermophilous deciduous stand), Chas. 887 (24/7/2010, $22.25683 \mathrm{E}, 41.15183 \mathrm{~N}, 1600 \mathrm{~m}$, grassy opening in beech stand) - Common. In various habitats, mainly in grasslands on stony ground, all-over the mountain.

*Orobanche amethystea Thuill. - T par, MA - Chas. 843 (26/6/2010, 22.21917 E, $41.1254 \mathrm{~N}, 1220 \mathrm{~m}$, open thermophilous deciduous shrubland on rocky slope), Chas. $2295(18 / 6 / 2013,22.23886 \mathrm{E}, 41.13768 \mathrm{~N}, 1510 \mathrm{~m}$, opening in low shrubland of Juniperus communis subsp. nana) - Common. Grasslands on stony ground, at low and mid-altitudes.

*Orobanche artemisiae-campestris Gaudin - T par, ME - Chas. 1264 (28/5/2011, $22.20433 \mathrm{E}, 41.10634 \mathrm{~N}, 700 \mathrm{~m}$, grassy opening in Juniperus oxycedrus thermophilous deciduous shrubland) - Rare.

*Orobanche caryophyllacea Sm. - T par, EA - Chas. 1179 (16/5/2011, 22.21678 E, $41.11275 \mathrm{~N}, 760 \mathrm{~m}$, opening with phrygana in thermophilous deciduous stand) Rare.

Orobanche crenata Forssk. - T par, ME - Voliotis 1983: 166.

*Orobanche elatior Sutton - T par, Pt - Chas. 1988 (4/6/2012, 22.24855 E, 41.1181 N, $760 \mathrm{~m}$, open shrubland of Juniperus oxycedrus and thermophilous deciduous), Chas. 1994 (8/6/2012, 22.22135 E, 41.11552 N, 870 m, phrygana with Juniperus 
oxycedrus) - Sporadic. Phrygana and open shrublands, on stony ground of lowland areas.

*Orobanche hederae Duby - T par, ME - Chas. 1502 (2/7/2011, 22.28024 E, 41.14524 $\mathrm{N}, 860 \mathrm{~m}$, streambank in mixed beech - thermophilous deciduous stand) - Rare.

*Orobanche minor Sm. - T par, EA - Chas. 717 (2/6/2010, 22.2118 E, 41.11186 N, 790 $\mathrm{m}$, road embankment in thermophilous deciduous stand), Chas. 1238 (25/5/2011, 22.2208 E, 41.10921 N, 640 m, dry grassland) - Sporadic. Dry grasslands and phrygana of lowland areas.

Orobanche pubescens d'Urv. - T par, Me - Voliotis 1983: 166.

Pedicularis leucodon Griseb. subsp. leucodon - H scap, Bk - Chas. 738 (12/6/2010, 22.17429 E, $41.15442 \mathrm{~N}, 1890 \mathrm{~m}$, open subalpine shrubland), Chas. 1378 (9/6/2011, 22.19228 E, 41.15894 N, 1870 m), MFG2: 248, Schuler 2004: tab. 21 Sporadic. Grasslands and open shrublands, on stony ground above treeline.

*Phelipanche nana (Reut.) Soják - T par, Pt - Chas. 1205 (20/5/2011, 22.22665 E, $41.11299 \mathrm{~N}, 740 \mathrm{~m}$, phrygana) - Sporadic. In dry grasslands and phrygana, of lowland areas.

*Rhinanthus sintenisii (Sterneck) Soó - T scap, Bk - Chas. 2216 (31/7/2012, 22.1732 E, $41.1553 \mathrm{~N}, 1950 \mathrm{~m}$, open subalpine shrubland) - Sporadic. In subalpine grasslands and open shrublands, on stony ground.

\section{OXALIDACEAE}

Oxalis acetosella L. - G rhiz, Bo - Chas. 1329 (2/6/2011, 22.23943 E, $41.14751 \mathrm{~N}$, $1650 \mathrm{~m}$, beech stand at treeline), Voliotis 1983: 166 - Sporadic in mesic beech stands at treeline. Rare in shrublands just above treeline.

\section{PAPAVERACEAE}

*Chelidonium majus L. - H scap, EA - Chas. 1706 (18/9/2011, 22.17884 E, 41.13657 N, $1150 \mathrm{~m}$, streambank in beech stand) - Rare.

*Papaver lecoqii Lamotte - T scap, EA - Chas. 664 (24/5/2010, 22.2634 E, 41.14238 $\mathrm{N}, 1150 \mathrm{~m}$, road in mixed beech - oak forest) - Rare.

Papaver rhoeas L. - T scap, Pt - Chas. 603 (19/5/2010, 22.21132 E, 41.11195 N, 790 $\mathrm{m}$, forest road in thermophilous deciduous stand), Voliotis 1983: 166 - Sporadic. In dry grasslands and forest roads of lowland areas.

\section{PARNASSIACEAE}

Parnassia palustris L. - H scap, ES - Chas. 249 (28/8/2009, 22.21436 E, 41.14046 N, 1590 m, damp place of road in beech forest), FH2: map 1328, Schuler 2004: tab. 20 - Sporadic. In damp places above treeline and forest roads in beech stands. Between 1550 and $1950 \mathrm{~m}$.

\section{PLANTAGINACEAE}

Plantago argentea Chaix - H ros, Eu - Chas. 855 (26/6/2010, 22.22409 E, 41.12845 N, $1440 \mathrm{~m}$, open thermophilous deciduous shrubland on rocky slope), Chas. 1334 (2/6/2011, 22.23124 E, $41.1372 \mathrm{~N}, 1670 \mathrm{~m}$, subalpine sparse grassland on stony ground), MFG2: 339 - Sporadic. Sparse grasslands on stony or rocky ground above and at treeline. 
Plantago atrata Hoppe subsp. atrata - H ros, Eu - Chas. 548 (8/5/2010, 22.22281 E, $41.14959 \mathrm{~N}, 1750 \mathrm{~m})$, Chas. 1536 (12/7/2011, $22.2298 \mathrm{E}, 41.1531 \mathrm{~N}, 1860 \mathrm{~m})$, MFG2: 337 - Common. Subalpine grasslands, mainly on stony ground.

*Plantago bellardii All. - T scap, Me - Chas. 1934 (30/5/2012, 22.19717 E, 41.11148 $\mathrm{N}, 850 \mathrm{~m}$, open shrubland of Juniperus oxycedrus and thermophilous deciduous), Chas. 2444 (28/6/2014, 22.2312 E, 41.11243 N, 690 m, dry grassland) - Sporadic. In open shrublands, phrygana and dry grasslands, of lowland areas.

*Plantago holosteum Scop. - H ros, Eu - Chas. 274 (28/8/2009, 22.21718 E, 41.16383 N, $1950 \mathrm{~m}$ ) - Common. Subalpine grasslands, on stony and eroded ground.

*Plantago lanceolata L. - H ros, Co - Chas. 212 (24/8/2009, 22.21376 E, 41.12306 N, $1100 \mathrm{~m}$, forest road in mixed beech - thermophilous deciduous stand), Chas. 250 $(28 / 8 / 2009,22.21258 \mathrm{E}, 41.1271 \mathrm{~N}, 1280 \mathrm{~m}$, grassy opening in beech stand), Chas. $1305(2 / 6 / 2011,22.21825 \mathrm{E}, 41.14178 \mathrm{~N}, 1590 \mathrm{~m}$, grassland at treeline) Common. Forest roads and grasslands on disturbed sites, between 600 and $1600 \mathrm{~m}$.

*Plantago major L. subsp. major - H ros, EA - Chas. 13 (11/7/2009, 22.18 E, 41.12893 N, 1000 m, forest road in beech stand), Chas. 74 (20/7/2009, 22.20764 E, 41.13774 $\mathrm{N}, 1700 \mathrm{~m}$, forest road in beech stand) - Common. At forest roads and grasslands on disturbed sites, between 600 and $1750 \mathrm{~m}$.

* Plantago media subsp. pindica (Hausskn.) Greuter \& Burdet - H ros, Bk - Chas. 2123 (30/6/2012, 22.21384 E, 41.16378 N, 2000 m), Chas. 2367 (10/7/2013, 22.21603 E, $41.15345 \mathrm{~N}, 1900 \mathrm{~m})$ - Sporadic. In streambanks above treeline.

\section{PLATANACEAE}

Platanus orientalis L. - P scap, EM - Chas. 1263 (28/5/2011, 22.20709 E, 41.10999 N, $780 \mathrm{~m}), \mathrm{FH} 2$ : map 1333 - Sporadic. In streams up to $900 \mathrm{~m}$.

\section{PLUMBAGINACEAE}

*Armeria rumelica Boiss. - H ros, Bk - Chas. 1561 (22/7/2011, 22.17674 E, 41.15203 $\mathrm{N}, 1750 \mathrm{~m}$, grassy opening of low shrubland at treeline on stony ground), Chas. $2098(30 / 6 / 2012,22.22507 \mathrm{E}, 41.15404 \mathrm{~N}, 1840 \mathrm{~m}$, subalpine sparse grassland on stony ground), Chas. 2391 (20/7/2013, 22.23797 E, $41.1573 \mathrm{~N}, 1680 \mathrm{~m}$, rocky streambank above treeline) - Sporadic. Stony places above treeline.

\section{POLYGALACEAE}

*Polygala comosa Schkuhr - H scap, Eu - Chas. 492 (25/4/2010, 22.20005 E, 41.10592 $\mathrm{N}, 700 \mathrm{~m}$, grassy opening in shrubland of Juniperus oxycedrus and thermophilous deciduous), Chas. 514 (2/5/2010, 22.20609 E, 41.1115 N, 820 m, Pteridium thicket), Chas. 604 (19/5/2010, 22.20858 E, 41.11208 N, 820 m, grassy opening in thermophilous deciduous stand), Chas. 1285 (28/5/2011, 22.20717 E, $41.11107 \mathrm{~N}$, 800 m, Pteridium thicket), Chas. 1931 (30/5/2012, 22.19818 E, 41.10886 N, 790 m, Platanus stand) - Sporadic. Pteridium thickets and grasslands of mesic ground, up to $900 \mathrm{~m}$.

Polygala major Jacq. - H scap, ES - Chas. 653 (24/5/2010, 22.24643 E, 41.15743 N, $1740 \mathrm{~m}$, subalpine grassland), Chas. 1139 (1/5/2011, 22.22698 E, 41.11435 N, 830 $\mathrm{m}$, shrubland of Juniperus oxycedrus and thermophilous deciduous), Voliotis 1983: 166 - Sporadic. Grasslands and shrublands on stony ground, up to $1900 \mathrm{~m}$.

Polygala nicaeensis subsp. mediterranea Chodat - H scap, Me - Chas. 378 (27/9/2009, 22.23095 E, $41.14386 \mathrm{~N}, 1990 \mathrm{~m}$, subalpine grassland on stony ground), MFG1: 
579, Schuler 2004: tab. 21 - Sporadic. On stony calcareous ground with grassy vegetation at mid-altitudes and above treeline.

*Polygala supina subsp. rhodopea (Velen.) McNeill - H scap, Bk - Chas. 1089 $(22 / 4 / 2011,22.23721 \mathrm{E}, 41.1191 \mathrm{~N}, 830 \mathrm{~m}$, rocky opening in thermophilous deciduous stand), Chas. 1140 (1/5/2011, 22.22788 E, 41.11598 N, 920 m, open shrubland of Juniperus oxycedrus and thermophilous deciduous), Chas. 1905 (24/5/2012, 22.23659 E, 41.10786 N, 620 m, phrygana with Juniperus oxycedrus) Sporadic. Juniperus oxycedrus shrublands and phrygana, on stony and rocky ground of lowland areas.

Polygala vulgaris L. - H scap, ES - Chas. 1220 (20/5/2011, 22.22724 E, 41.11336 N, $760 \mathrm{~m}$, phrygana), Chas. $1250(25 / 5 / 2011,22.21635 \mathrm{E}, 41.11986 \mathrm{~N}, 1110 \mathrm{~m}$, shrubland of thermophilous deciduous and Juniperus oxycedrus), Chas. 1333 $(2 / 6 / 2011,22.23383 \mathrm{E}, 41.13841 \mathrm{~N}, 1700 \mathrm{~m}$, subalpine grassland on stony ground), Voliotis 1983: 166 - Common. Grasslands, phrygana and shrublands, on stony ground. $700-2050 \mathrm{~m}$.

\section{POLYGONACEAE}

*Aconogonon alpinum (All.) Tzvelev. - H scap, Pt - Chas. 2211 (31/7/2012, 22.17061 E, 41.15391 N, 2090 m, subalpine grassland) - Rare. In subalpine grasslands and open shrublands, on siliceous substrates.

*Fallopia convolvulus (L.) Á. Löve - T scand/H scand, Ct - Chas. 1740 (19/10/2011, $22.22833 \mathrm{E}, 41.12149 \mathrm{~N}, 1190 \mathrm{~m}$, grassy opening in thermophilous deciduous stand), Chas. 2203 (27/7/2012, $22.2589 \mathrm{E}, 41.12772 \mathrm{~N}, 810 \mathrm{~m}$, forest road in Quercus frainetto stand) - Rare. Grasslands and forest roads, up to $1250 \mathrm{~m}$.

*Fallopia dumetorum (L.) J. Holub - T scand/H scand, EA - Chas. 1679 (10/9/2011, 22.2727 E, $41.15567 \mathrm{~N}, 1270 \mathrm{~m}$, forest road in beech stand) - Rare.

*Persicaria lapathifolia (L.) S. F. Gray subsp. lapathifolia - T scap, Ct - Chas. 962 $(26 / 9 / 2010,22.27048 \mathrm{E}, 41.1405 \mathrm{~N}, 1020 \mathrm{~m}$, forest road in mixed oak and beech stand) - Rare.

*Polygonum aviculare subsp. neglectum (Besser) Arcang. - T rept, EA - Chas. 964 $(26 / 9 / 2010,22.26282 \mathrm{E}, 41.14156 \mathrm{~N}, 1140 \mathrm{~m}$, forest road in mixed oak - beech stand), Chas. 2101 (30/6/2012, 22.22214 E, 41.14805 N, $1790 \mathrm{~m}$, subalpine grassland at disturbed site) - Sporadic. Forest roads and other disturbed sites. 600 $1800 \mathrm{~m}$.

Rumex acetosella subsp. acetoselloides (Balansa) Nijs - H scap, EA - Chas. 765 $(12 / 6 / 2010,22.17528 \mathrm{E}, 41.15759 \mathrm{~N}, 1900 \mathrm{~m}$, grassy opening in subalpine shrubland), Chas. 1276 (28/5/2011, 22.19337 E, 41.11509 N, $910 \mathrm{~m}$, forest road in thermophilous deciduous stand), Chas. 1496 (2/7/2011, 22.27738 E, $41.14583 \mathrm{~N}$, $980 \mathrm{~m}$, Quercus frainetto stand), FH1: map 163 - Common in subalpine grasslands on stony, eroded ground and at roads above treeline. Sporadic in the forest zone in similar habitats.

*Rumex alpinus L. - G rhiz, EA - obs. (15/7/2013, 22.24848 E, 41.16125 N, 1820 m, tall-herb vegetation above treeline) - Rare.

*Rumex arifolius All. - H scap, Eu - Chas. 125 (6/8/2009, 22.18389 E, 41.15205 N, $1600 \mathrm{~m}$, forest road in beech stand), Chas. $1651(23 / 8 / 2011,22.21779 \mathrm{E}, 41.16339$ N, 1930 m, subalpine shrubland), Chas. 2117 (30/6/2012, 22.21372 E, 41.16396 N, 2010 m, subalpine grassland) - Common. Grasslands, shrublands and roads above 
treeline and forest openings and roads at the higher altitudes of beech forest zone. $1400-2100 \mathrm{~m}$.

*Rumex conglomeratus Murray - H scap, EA - Chas. 109 (27/7/2009, 22.1844 E, $41.1335 \mathrm{~N}, 1190 \mathrm{~m}$, forest road in beech stand) - Rare.

*Rumex crispus L. - H scap, Co - Chas. 1957 (4/6/2012, 22.20377 E, 41.11786 N, 980 $\mathrm{m}$, damp grassy clearing in thermophilous deciduous stand) - Rare.

*Rumex obtusifolius L. - H scap, EA - Chas. 2088 (25/6/2012, 22.21413 E, 41.13991 $\mathrm{N}, 1560 \mathrm{~m}$, forest road in beech stand) - Sporadic. Forest roads in mesic oak and beech forests.

*Rumex pulcher L. - H scap, MS - Chas. 1912 (24/5/2012, 22.22707 E, 41.11102 N, $660 \mathrm{~m}$, grassland), Chas. $2246(1 / 6 / 2013,22.26676 \mathrm{E}, 41.12757 \mathrm{~N}, 720 \mathrm{~m}$ grassland) - Rare. Grasslands and forest roads on mesic ground of lowland areas.

*Rumex scutatus L. - H scap, EA - Chas. 123 (6/8/2009, 22.18389 E, 41.15205 N, $1600 \mathrm{~m}$, forest road in beech stand), Chas. 253 (28/8/2009, $22.21319 \mathrm{E}, 41.14404$ $\mathrm{N}, 1760 \mathrm{~m}$, rocky opening in subalpine shrubland) - Sporadic. Forest roads and subalpine grasslands on stony ground, between 1200 and $2000 \mathrm{~m}$.

\section{PRIMULACEAE}

*Anagallis arvensis L. - $\mathrm{T}$ rept/H rept, Co - Chas. $829(26 / 6 / 2010,22.22452 \mathrm{E}$, $41.11269 \mathrm{~N}, 700 \mathrm{~m}$, dry grassland) - Sporadic. In dry grasslands and phrygana. 600 - $800 \mathrm{~m}$.

Androsace villosa L. - H rept, EA - Chas. 582 (12/5/2010, 22.24691 E, 41.14402 N, $1660 \mathrm{~m}$ ), Voliotis 1983: 166, MFG1: 744 - Sporadic. At rocky and stony calcareous places with sparse vegetation above and at treeline.

Cyclamen hederifolium Aiton - G bulb, Me - Chas. 373 (27/9/2009, 22.20122 E, $41.11098 \mathrm{~N}, 850 \mathrm{~m}$, thermophilous deciduous stand), Voliotis 1983: 166 Common. In forests and shrublands up to $1500 \mathrm{~m}$.

*Lysimachia punctata L. - H scap, Eu - Chas. 30 (11/7/2009, 22.18 E, 41.12893 N, $1000 \mathrm{~m}$, forest road in beech stand), Chas. 792 (12/6/2010, 22.18844 E, 41.12987 $\mathrm{N}, 1230 \mathrm{~m}$, forest road in beech stand) - Sporadic. At damp places of forest roads and forest openings, in beech forests.

Primula elatior (L.) L. - H ros, EA - Chas. 536 (8/5/2010, 22.2301 E, 41.16214 N, $1800 \mathrm{~m}$, streambank above treeline), Chas. 1046 (11/4/2011, $22.20148 \mathrm{E}, 41.13215$ $\mathrm{N}, 1600 \mathrm{~m}$, streambank in beech forest), Chas. $1773(19 / 4 / 2012,22.20377 \mathrm{E}$, $41.13119 \mathrm{~N}, 1520 \mathrm{~m}$, streambank in beech forest), Chas. 1804 (2/5/2012, 22.21649 E, $41.14085 \mathrm{~N}, 1570 \mathrm{~m}$, streambank in beech forest), Voliotis 1983: 166 (as $P$. elatior subsp. intricata (Gren. \& Godr.) Lüdi) - Sporadic. At streambanks, above treeline and in beech forests, between 1450 and $2050 \mathrm{~m}$.

Primula veris $\mathrm{L}$. subsp. veris $-\mathrm{H}$ ros, $\mathrm{Eu}$ - Chas. 439 (10/4/2010, 22.22918 E, $41.12537 \mathrm{~N}, 1320 \mathrm{~m}$, thermophilous deciduous stand), Chas. $524(2 / 5 / 2010$, 22.1895 E, 41.1216 N, $1070 \mathrm{~m}$, beech stand), Chas. 554 (8/5/2010, 22.22256 E, $41.15013 \mathrm{~N}, 1750 \mathrm{~m}$, subalpine grassland), Voliotis 1983: 166 - Common. In forests and grasslands of mesic grounds, between 600 and $2000 \mathrm{~m}$.

Primula vulgaris Huds. subsp. vulgaris - H ros, EA - Chas. 458 (17/4/2010, 22.22105 E, $41.11777 \mathrm{~N}, 850 \mathrm{~m}$, streambank in mixed thermophilous deciduous - beech stand), Voliotis 1983: 166 - Common. Mesic to damp ground in forests, up to 1500 m. 


\section{RANUNCULACEAE}

*Actaea spicata L. - G rhiz, ES - Chas. 2193 (15/7/2012, 22.24241 E, 41.15638 N, $1650 \mathrm{~m})$ - Rare. in beech stands at treeline.

Adonis flammea Jacq. - T scap, EA - Chas. 1809 (2/5/2012, 22.218 E, 41.107 N, 610 $\mathrm{m}$, dry grassland), Voliotis 1983: 166 - Rare.

Anemone pavonina Lam. - G bulb, Me - Chas. 440 (10/4/2010, 22.23015 E, 41.11602 $\mathrm{N}, 880 \mathrm{~m}$ ), Voliotis 1983: 166 - Common. In grasslands and open shrublands, on stony ground, up to $1300 \mathrm{~m}$.

Anemone ranunculoides L. - G rhiz, ES - Chas. 535 (8/5/2010, 22.24072 E, 41.15698 $\mathrm{N}, 1660 \mathrm{~m}$, beech stand at treeline), Chas. 988 (28/3/2011, 22.19177 E, $41.11272 \mathrm{~N}$, $720 \mathrm{~m}$, swampy place with alders), Voliotis 1983: 166, MFG1: 208 - Common. Pteridium thickets and beech forests on mesic to damp ground, between 1100 and $1800 \mathrm{~m}$, rarely at lower altitudes.

*Clematis vitalba L. - P lian, EA - Chas. 308 (10/9/2009, 22.25447 E, 41.13756 N, $1210 \mathrm{~m}$, forest road in beech stand) - Common. In forests, shrublands and roadsides, between 600 and $1400 \mathrm{~m}$.

Consolida regalis subsp. paniculata (Host) Soó - T scap, EA - Chas. 2158 (6/7/2012, 22.28536 E, 41.14072 N, 750 m, grassland within Quercus frainetto stand), Voliotis 1983: 167 - Rare.

*Delphinium balcanicum Pawl. - T scap, Bk - Chas. 2181 (11/7/2012, 22.21561 E, $41.11183 \mathrm{~N}, 740 \mathrm{~m}$, forest road in thermophilous deciduous and Juniperus oxycedrus shrubland) - Rare.

*Delphinium fissum Waldst. \& Kit. subsp. fissum - H scap, EA - Chas. 882 (24/7/2010, 22.24587 E, 41.14725 N, $1540 \mathrm{~m}$, grassy opening in beech stand) Sporadic. Grasslands and thermophilous deciduous stands, on stony calcareous ground between 900 and $1600 \mathrm{~m}$.

?Ficaria verna subsp. bulbifera Á. Löve \& D. Löve - G bulb, ES - Voliotis 1983: 166.

*Ficaria verna subsp. ficariiformis (F.W. Schultz) B. Walln. - G bulb, Me - Chas. 989 (28/3/2011, 22.19133 E, $41.11578 \mathrm{~N}, 890 \mathrm{~m}$, thermophilous deciduous stand), Chas. 1805 (2/5/2012, 22.21649 E, $41.14085 \mathrm{~N}, 1570 \mathrm{~m}$, beech stand at treeline) Sporadic. Mesic forests.

*Nigella arvensis L. subsp. arvensis - T scap, ME - Chas. 1408 (16/6/2011, 22.22684 E, $41.11507 \mathrm{~N}, 860 \mathrm{~m}$, grassy opening in shrubland of Juniperus oxycedrus and thermophilous deciduous) - Sporadic. Dry grasslands, shrubland openings and forest roads of lowland areas.

*Ranunculus acris L. - H scap, ES - Chas. 384 (27/9/2009, 22.20742 E, 41.1372 N, $1680 \mathrm{~m}$, ditch by roadside in beech stand), Chas. $468(17 / 4 / 2010,22.21978 \mathrm{E}$, 41.11622 N, 920 m), Chas. 693 (2/6/2010, 22.19095 E, 41.12946 N, 1290 m, ditch by roadside in beech stand), Chas. 2424 (26/5/2014, 22.27504 E, $41.15282 \mathrm{~N}, 1100$ $\mathrm{m}$, opening in beech stand) - Sporadic. Forest roads, grasslands and forest openings of mesic beech stands. $800-1700 \mathrm{~m}$.

Ranunculus illyricus L. - H scap, EA - Chas. 2016 (13/6/2012, 22.22861 E, 41.11998 $\mathrm{N}, 1110 \mathrm{~m}$ ), Chasapis et al. 2019 - Rare. Grasslands within thermophilous deciduous stands, on calcareous ground.

*Ranunculus millefoliatus Vahl - H scap, ME - Chas. 486 (25/4/2010, 22.19832 E, $41.10823 \mathrm{~N}, 770 \mathrm{~m}$, mesic grassland), Chas. 1153 (6/5/2011, 22.26943 E, 41.14859 $\mathrm{N}, 1250 \mathrm{~m}$, grassy opening in beech stand) - Sporadic. Grasslands and forest openings on mesic or damp ground, between 650 and $1300 \mathrm{~m}$. 
*Ranunculus neapolitanus Ten. - H scap, Me - Chas. 2427 (9/6/2014, 22.22503 E, 41.11153 N, $660 \mathrm{~m}$, Platanus stand) - Rare. Mesic to damp forests or grasslands of lowland areas.

*Ranunculus platanifolius L. - H scap/G rhiz, Eu - Chas. 746 (12/6/2010, 22.18087 E, 41.14948 N, $1650 \mathrm{~m})$, Chas. $1180(16 / 5 / 2011,22.18603$ E, 41.15226 N, $1650 \mathrm{~m})$ Rare. Beech stands below the treeline, on siliceous substrates.

Ranunculus polyanthemos subsp. polyanthemoides (Boreau) Ahlfv. - H scap, Eu Chas. $2323(22 / 6 / 2013,22.19488$ E, $41.15555 \mathrm{~N}, 1810 \mathrm{~m}$, above treeline fen), Voliotis 1983: 167 - Rare.

*Ranunculus polyanthemos L. subsp. polyanthemos - H scap, EA - Chas. 1450 (22/6/2011, 22.20728 E, 41.11143 N, 820 m, Pteridium thicket), Chas. 1919 (30/5/2012, 22.19831 E, 41.10859 N, 780 m, streambank in Platanus stand) Sporadic. In Pteridium thickets, grasslands and Platanus stands, on damp or mesic ground, of lowland areas.

Ranunculus psilostachys Griseb. - H scap, Bk - Chas. 575 (12/5/2010, 22.24867 E, $41.13594 \mathrm{~N}, 1250 \mathrm{~m}$, mixed beech - oak stand), Chas. 1799 (29/4/2012, 22.20682 E, 41.11097 N, 800 m, Pteridium thicket), MFG1: 217 - Sporadic. Grasslands, Pteridium thickets and forests, between 800 and $1400 \mathrm{~m}$.

*Ranunculus repens L. - H rept, Pt - Chas. 2126 (30/6/2012, 22.218 E, 41.14682 N, $1800 \mathrm{~m}$, ditch by roadside above treeline), Chas. 2261 (5/6/2013, 22.21764 E, $41.13375 \mathrm{~N}, 1320 \mathrm{~m}$, damp place in beech stand) - Rare. Damp places in forests.

Ranunculus sartorianus Boiss. \& Heldr. - G rhiz, BA - Chas. 545 (8/5/2010, 22.22281 E, $41.14959 \mathrm{~N}, 1760 \mathrm{~m}$, subalpine grassland), Chas. 1364 (9/6/2011, 22.19703 E, $41.15108 \mathrm{~N}, 1720 \mathrm{~m}$, grassy opening in subalpine shrubland), Voliotis 1983: 167, MFG1: 214, FH2: map 794 - Common. Subalpine grasslands and grassy openings in beech forests, between 1450 and $2180 \mathrm{~m}$.

*Ranunculus serbicus Vis. - G rhiz, BI - Chas. 385 (27/9/2009, 22.20933 E, 41.13686 $\mathrm{N}, 1610 \mathrm{~m}$, ditch by roadside in beech stand), Chas. 2047 (21/6/2012, 22.21291 E, $41.12782 \mathrm{~N}, 1360 \mathrm{~m}$, ditch by roadside in beech stand) - Rare. Damp places in beech forests.

*Ranunculus sprunerianus Boiss. - H scap, EM - Chas. 505 (2/5/2010, 22.22395 E, 41.11187 N, 680 m, dry grassland), Chas. 605 (19/5/2010, 22.20977 E, $41.11201 \mathrm{~N}$, $810 \mathrm{~m}$, open shrubland of thermophilous deciduous and Juniperus oxycedrus) Common. Phrygana, open shrublands and dry grasslands, on stony or rocky calcareous ground. $600-1400 \mathrm{~m}$.

*Ranunculus trichophyllus Chaix - T scap/ H scap, Ct/[Co] - Chas. obs. (31/5/2014, 22.20927 E, 41.12597 N, 1200 m, swamp in beech stand) - Rare.

*Thalictrum aquilegiifolium L. - Grhiz, Eu - Chas. 750 (12/6/2010, 22.19164 E, $41.15208 \mathrm{~N}, 1580 \mathrm{~m}$, opening in beech stand at treeline), Chas. 1191 (20/5/2011, 22.22396 E, $41.11592 \mathrm{~N}, 780 \mathrm{~m}$, thermophilous deciduous stand) - Sporadic. Pteridium thickets and beech forest openings at treeline, rarely at lower altitudes.

*Thalictrum lucidum L. - G rhiz, Eu - Chas. 2174 (11/7/2012, 22.20287 E, 41.11154 $\mathrm{N}, 860 \mathrm{~m}$, grassy opening on damp ground in thermophilous deciduous stand) Rare.

Thalictrum minus subsp. saxatile Hooker fil. - G rhiz, ME - Chas. 888 (24/7/2010,

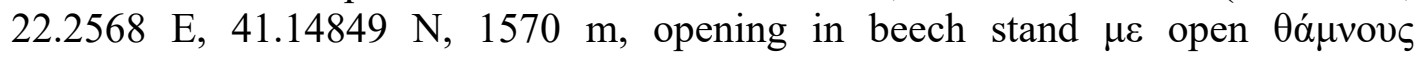
Juniperus communis subsp. nana), Chas. 2011 (13/6/2012, 22.22861 E, 41.11998 N, $1110 \mathrm{~m}$, grassland in shrubland thermophilous deciduous and Juniperus oxycedrus), 
FH2: map 852, MFG1: 229 - Common. Grasslands and open shrublands, on stony calcareous ground. $800-1800 \mathrm{~m}$.

\section{$\underline{R E S E D A C E A E}$}

Reseda lutea L. - T scap/ H scap, Pt - Chas. 210 (24/8/2009, 22.21376 E, 41.12306 N, $1100 \mathrm{~m}$, rocky roadside in mixed beech and thermophilous deciduous stand), Voliotis 1983: 167, FH2: map 1245 - Sporadic. At forest roads of lowland areas.

*Reseda luteola L. - H scap, EA - Chas. 1668 (31/8/2011, 22.17619 E, 41.14607 N, $1450 \mathrm{~m}$, forest road in beech stand) - Rare.

*Reseda phyteuma L. - T scap/H scap, ME - Chas. 1778 (22/4/2012, 22.22115 E, $41.1094 \mathrm{~N}, 640 \mathrm{~m}$, forest road in dry grassland) - Sporadic. At forest roads of lowland areas.

\section{$\underline{\text { RHAMNACEAE }}$}

*Frangula rupestris (Scop.) Schur - P caesp, BI - Chas. 827 (26/6/2010, 22.21801 E, $41.1243 \mathrm{~N}, 1120 \mathrm{~m}$, open thermophilous deciduous shrubland on rocky slope) Rare. On rocky calcareous places with open shrublands.

*Paliurus spina-christi Miller - P caesp, EA - Chas. 1355 (9/6/2011, 22.21812 E, 41.10692 N, 610 m, dry grassland) - Rare. In dry grasslands and open shrublands, between 600 and $750 \mathrm{~m}$.

Rhamnus alpina subsp. fallax (Boiss.) Maire \& Petitmengin - P caesp, EM - Chas. 880 (24/7/2010, 22.24752 E, 41.14808 N, 1570 m), Chas. 1321 (2/6/2011, 22.22358 E, $41.1342 \mathrm{~N}, 1470 \mathrm{~m})$, MFG1: 587 - Sporadic. Beech stands on stony calcareous ground, between 1400 and $1700 \mathrm{~m}$.

Rhamnus pumila Turra - P caesp, Me - MFG1: 588.

*Rhamnus saxatilis subsp. rhodopea (Velen.) Aldén - P caesp, BA - Chas. 970 (31/10/2010, $22.23174 \mathrm{E}, 41.11884 \mathrm{~N}, 970 \mathrm{~m}$, rocky place with sparse phrygana), Chas. 1714 (24/9/2011, 22.23603 E, 41.12773 N, 1190 m, open Juniperus oxycedrus shrubland) - Sporadic. Rocky and stony calcareous places with sparse vegetation, up to $1300 \mathrm{~m}$.

\section{ROSACEAE}

*Agrimonia eupatoria L. subsp. eupatoria - H scap, EA - Chas. 177 (13/8/2009, 22.18554 E, 41.12244 N, $950 \mathrm{~m}$, opening in beech stand), Chas. 2147 (6/7/2012, 22.2916 E, 41.14838 N, 830 m, opening in oak forest) - Sporadic. In grasslands and openings forests, of lowland areas.

*Alchemilla acutiloba Opiz - H ros, ES - Chas. 2389 (20/7/2013, 22.2391 E, 41.15525 N, 1750 m), Chas. 2407 (25/7/2013, 22.2148 E, 41.1637 N, 1990 m) - Sporadic. Above treeline fens.

*Alchemilla bulgarica Rothm. - H ros, Bk - Chas. 2377 (15/7/2013, 22.24953 E, $41.16207 \mathrm{~N}, 1890 \mathrm{~m}$, tall-herb vegetation above treeline) - Rare.

Alchemilla indivisa Formánek - H ros, Bk - Chas. 54 (20/7/2009, 22.21366 E, $41.14405 \mathrm{~N}, 1760 \mathrm{~m}$, damp place in subalpine shrubland), Chas. 776 (12/6/2010, $22.18995 \mathrm{E}, 41.12683 \mathrm{~N}, 1210 \mathrm{~m}$, ditch by roadside in beech stand), MFG1: 428, Schuler 2004: tab. 20 - Common in fens and other damp places above treeline. Rare in streambanks and ditches by roadsides in beech stands. 
Alchemilla lanuginosa Rothm. - H ros, Bk - Chas. 747 (12/6/2010, 22.17554 E, $41.1566 \mathrm{~N}, 1860 \mathrm{~m})$, Chas. 2196 (15/7/2012, $22.24076 \mathrm{E}, 41.15554 \mathrm{~N}, 1710 \mathrm{~m})$, MFG1: 420 - Sporadic. In grasslands and shrubland openings above treeline.

*Alchemilla monticola Opiz - H ros, ES - Chas. 2335 (26/6/2013, 22.23103 E, $41.15855 \mathrm{~N}, 1690 \mathrm{~m}$, tall-herb vegetation above treeline), Chas. 2408 (25/7/2013, 22.2148 E, 41.1637 N, 1990 m, above treeline fen) - Sporadic. Tall-herb meadows and fens, above treeline.

*Alchemilla obtusa Buser - H ros, Eu - Chas. 2050 (21/6/2012, 22.22022 E, 41.14406 $\mathrm{N}, 1660 \mathrm{~m}$, streambank above treeline), Chas. 2140 (30/6/2012, $22.22149 \mathrm{E}$, $41.15885 \mathrm{~N}, 1850 \mathrm{~m}$, above treeline fen), Chas. $2189(15 / 7 / 2012,22.24099 \mathrm{E}$, $41.15582 \mathrm{~N}, 1700 \mathrm{~m}$, rivulet above treeline), Chas. 2406 (25/7/2013, 22.2148 E, $41.1637 \mathrm{~N}, 1990 \mathrm{~m}$, above treeline fen), Chas. 2420 (30/7/2013, 22.21 E, 41.16471 $\mathrm{N}, 2050 \mathrm{~m}$, tall-herb vegetation above treeline) - Common. Mesic and damp ground above treeline.

Alchemilla serbica (Paulin) Pawł. - H ros, Bk - Chas. 743 (12/6/2010, 22.17535 E, 41.15507 N, $1840 \mathrm{~m})$, Chas. 2065 (21/6/2012, $22.22316 \mathrm{E}, 41.14591 \mathrm{~N}, 1780 \mathrm{~m})$, MFG1: 420 - Sporadic. In grasslands and shrubland openings above treeline.

*Alchemilla xanthochlora Rothm. - H ros, Eu - Chas. 2141 (30/6/2012, 22.22149 E, $41.15885 \mathrm{~N}, 1850 \mathrm{~m}$, swamp above treeline) - Sporadic. At mesic and damp ground above treeline.

Amelanchier ovalis Medik. subsp. ovalis - P caesp, ME - Chas. 826 (26/6/2010, 22.21801 E, $41.1243 \mathrm{~N}, 1120 \mathrm{~m}$, open shrubland of thermophilous deciduous on rocky slope), Chas. $1169(11 / 5 / 2011,22.21925 \mathrm{E}, 41.12243 \mathrm{~N}, 990 \mathrm{~m}$, thermophilous deciduous shrubland on stony ground), MFG1: 436 - Sporadic. Rocky and stony calcareous places in shrublands.

Aremonia agrimonoides (L.) DC. subsp. agrimonoides - H ros, BC - Chas. 501 $(25 / 4 / 2010,22.18587$ E, $41.11929 \mathrm{~N}, 910 \mathrm{~m}$, mixed beech - thermophilous deciduous stand), Chas. 1368 (9/6/2011, $22.19537 \mathrm{E}, 41.15061 \mathrm{~N}, 1670 \mathrm{~m}$, subalpine shrubland), Voliotis 1983: 167 - Common in forests all-over the mountain. Rare in shrublands at and above treeline.

Cotoneaster integerrimus Medik. - P caesp, EA - Chas. 237 (28/8/2009, 22.21319 E, $41.14404 \mathrm{~N}, 1760 \mathrm{~m})$, Chas. $674(24 / 5 / 2010,22.25831 \mathrm{E}, 41.15878 \mathrm{~N}, 1560 \mathrm{~m})$, MFG1: 438 - Sporadic. In shrublands on stony calcareous ground. $1400-2000 \mathrm{~m}$.

Cotoneaster tomentosus (Aiton) Lindl. - P caesp, ME - Chas. 340 (15/9/2009, 22.25928 E, $41.16285 \mathrm{~N}, 1750 \mathrm{~m}$, subalpine shrubland), Chas. 848 (26/6/2010, 22.22221 E, $41.1277 \mathrm{~N}, 1370 \mathrm{~m}$, open thermophilous deciduous shrubland on rocky slope), MFG1: 439 - Common. Shrublands on stony calcareous ground. 1300 $2000 \mathrm{~m}$.

*Crataegus monogyna Jacq. - P caesp, Pt - Chas. 1185 (16/5/2011, 22.21836 E, $41.1071 \mathrm{~N}, 620 \mathrm{~m}$, dry grassland) - Rare.

Dryas octopetala L. - C rept, AA - Chas. 381 (27/9/2009, 22.23224 E, 41.1446 N, 1890 $\mathrm{m}$, rocky place with sparse vegetation above treeline), Chas. $1628(18 / 8 / 2011$, 22.2297 E, 41.14574 N, 1930 m, open subalpine shrubland), MFG1: 401, Schuler 2004: tab. 21 - Rare. At the north and north-east slope of summit "Tzena", between 1850 and $2000 \mathrm{~m}$, on calcareous stony or rocky ground with usually sparse vegetation. 
Filipendula vulgaris Moench - H scap, ES - Chas. 851 (26/6/2010, 22.22752 E, $41.12967 \mathrm{~N}, 1550 \mathrm{~m})$, Chas. 1902 (24/5/2012, $22.23548 \mathrm{E}, 41.10799 \mathrm{~N}, 630 \mathrm{~m})$, Voliotis 1983: 167 - Common. In grasslands, from $600 \mathrm{~m}$ up to the treeline.

Fragaria vesca L. - H rept, EA - Chas. 512 (2/5/2010, 22.21113 E, 41.11897 N, 990 m, mixed beech and thermophilous deciduous stand), Voliotis 1983: 167 - Common. In beech and oak forests, Pteridium thickets and grasslands of mid- to high altitudes and in shrublands above treeline.

*Fragaria viridis Weston subsp. viridis - H rept, ES - Chas. 1962 (4/6/2012, 22.20777 E, 41.11126 N, 820 m, Pteridium thicket) - Rare.

Geum coccineum Sibth. \& Sm. - H scap, BA - Chas. $671(24 / 5 / 2010,22.25907$ E, $41.16186 \mathrm{~N}, 1690 \mathrm{~m})$, MFG1: 404 - Common. In grasslands, on mesic to damp ground above treeline.

Geum montanum L. - H ros, Eu - Chas. 919 (1/8/2010, 22.21145 E, 41.15969 N, 2140 m), Chas. 1858 (16/5/2012, 22.22623 E, 41.15532 N, 1810 m), MFG1: 402 Sporadic. In subalpine grasslands on siliceous substrates.

*Geum urbanum L. - H scap, EA - Chas. 25 (11/7/2009, 22.18 E, 41.12893 N, 1000 m, beech stand), Chas. 694 (2/6/2010, 22.19095 E, 41.12946 N, 1290 m, forest road in beech stand) - Sporadic. In mesic beech and oak forests.

*Malus sylvestris (L.) Mill. - P scap, Eu - Chas. 961 (26/9/2010, 22.2706 E, 41.14924 N, $1230 \mathrm{~m}$, forest road in beech stand), Chas. 1128 (1/5/2011, 22.20977 E, 41.1121 $\mathrm{N}, 820 \mathrm{~m}$, open thermophilous deciduous stand) - Rare.

*Potentilla argentea L. - H scap, ES - Chas. 1271 (28/5/2011, 22.18828 E, 41.11825 $\mathrm{N}, 980 \mathrm{~m}$, opening with disturbed ground in mixed beech - thermophilous deciduous stand) - Sporadic. Forest roads and dry grasslands, at lowland areas.

*Potentilla aurea subsp. chrysocraspeda (Lehm.) Nyman - H scap, ME - Chas. 1367 (9/6/2011, 22.19537 E, $41.15061 \mathrm{~N}, 1670 \mathrm{~m}$, subalpine shrubland) - Sporadic. Grasslands and shrublands, above treeline, on siliceous substrates. $1650-2180 \mathrm{~m}$.

*Potentilla detommasii Ten. - H scap, BI - Chas. 2442 (28/6/2014, 22.2312 E, $41.11243 \mathrm{~N}, 690 \mathrm{~m}$ ) - Sporadic. In dry grasslands of lowland areas.

Potentilla erecta (L.) Räusch. - H scap, ES - Chas. 267 (28/8/2009, 22.21367 E, $41.14387 \mathrm{~N}, 1740 \mathrm{~m}$, damp place by roadside above treeline), Chas. 1312 $(2 / 6 / 2011,22.21895 \mathrm{E}, 41.14233 \mathrm{~N}, 1610 \mathrm{~m}$, streambank at treeline), Strid \& Franzèn 1982: 12, Schuler 2004: tab. 21 - Sporadic. Damp places above treeline.

*Potentilla inclinata Vill. - H scap, EA - Chas. 795 (12/6/2010, 22.18462 E, 41.14287 $\mathrm{N}, 1470 \mathrm{~m}$, roadside in beech stand), Chas. 1281 (28/5/2011, 22.20682 E, 41.11088 N, $800 \mathrm{~m}$, Pteridium thicket) - Sporadic. Grasslands, Pteridium thickets and roads, up to $1500 \mathrm{~m}$.

Potentilla micrantha DC. - Hscap, EA - Chas. 978 (13/3/2011, 22.20848 E, 41.1155 N, $930 \mathrm{~m}$ ), Voliotis 1983: 167 - Common. In forests and shrublands up to $1800 \mathrm{~m}$.

*Potentilla recta subsp. laciniosa (Nestl.) Nyman - H scap, ME - Chas. 2430 (19/6/2014, 22.27387 E, 41.1292 N, 710 m, oak forest) - Rare.

Potentilla recta L. subsp. recta - H scap, EA - Chas. $72(20 / 7 / 2009,22.22324$ E, $41.14699 \mathrm{~N}, 1800 \mathrm{~m})$, Chas. $361(15 / 9 / 2009,22.25928 \mathrm{E}, 41.16285 \mathrm{~N}, 1750 \mathrm{~m})$, Chas. 1262 (28/5/2011, 22.2031 E, 41.10776 N, 740 m), MFG1: 410 - Common. Grasslands. 800 - $1850 \mathrm{~m}$.

*Potentilla reptans L. - H ros, Pt - Chas. 1593 (13/8/2011, 22.22671 E, 41.11101 N, $660 \mathrm{~m}$, Prunus spinosa stand) - Rare. 
*Prunus avium (L.) L. - P scap, EA - Chas. 791 (12/6/2010, 22.18866 E, 41.13474 N, $1360 \mathrm{~m}$, road embankment in beech stand) - Rare. in forests, up to $1500 \mathrm{~m}$.

*Prunus cerasifera Ehrh. - P caesp/P scap, EA - Chas. 89 (27/7/2009, 22.18677 E, 41.12976 N, $1200 \mathrm{~m}$, opening in beech stand), Chas. 117 (6/8/2009, $22.20882 \mathrm{E}$, $41.112 \mathrm{~N}, 810 \mathrm{~m}$, forest road in thermophilous deciduous stand) - Common. In forests, mainly forest openings, up to $1600 \mathrm{~m}$.

*Prunus spinosa L. - P caesp, EA - Chas. 1356 (9/6/2011, 22.20965 E, 41.11201 N, $810 \mathrm{~m}$, opening in thermophilous deciduous stand) - Sporadic. Open shrublands and dry grasslands. $600-850 \mathrm{~m}$.

*Pyracantha coccinea M. Roem. - P caesp, ME - Chas. 969 (31/10/2010, 22.22952 E, $41.11286 \mathrm{~N}, 720 \mathrm{~m}$ ) - Rare. In thermophilous deciduous shrublands, up to $800 \mathrm{~m}$.

*Pyrus pyraster (L.) Burgsd. - P scap, ME - Chas. 1042 (11/4/2011, 22.19602 E, $41.11387 \mathrm{~N}, 900 \mathrm{~m}$, road embankment in thermophilous deciduous stand), Chas. 1766 (5/4/2012, 22.21952 E, 41.10829 N, 630 m, dry grassland) - Rare. in forest roads and grasslands of lowland areas.

*Pyrus spinosa Forssk. - P caesp/P scap, Me - Chas. 1449 (22/6/2011, 22.20772 E, $41.11279 \mathrm{~N}, 840 \mathrm{~m}$, grassy opening in thermophilous deciduous stand) - Sporadic. Forest openings and grasslands, up to $1400 \mathrm{~m}$.

*Rosa agrestis Savi - P caesp, ME - Chas. 1932 (30/5/2012, 22.197 E, 41.10875 N, $760 \mathrm{~m}$, shrubland of Juniperus oxycedrus and thermophilous deciduous) - Rare. Shrublands of lowland areas.

*Rosa arvensis Huds. - P caesp, Me - Chas. 1436 (22/6/2011, 22.21024 E, 41.11555 N, $910 \mathrm{~m}$, thermophilous deciduous stand) - Common. Oak forests, thermophilous deciduous forests and beech forests at the lower parts of the beech forest zone.

* Rosa canina L. - P caesp, Pt - Chas. 175 (13/8/2009, 22.18554 E, 41.12244 N, 950 m, opening in beech stand), Chas. 1509 (2/7/2011, 22.27636 E, $41.14955 \mathrm{~N}, 1030 \mathrm{~m}$, Quercus frainetto stand), Chas. 1860 (16/5/2012, 22.22069 E, 41.10903 N, 640 m, dry grassland), Chas. 1886 (24/5/2012, 22.20784 E, 41.1127 N, 840 m, opening in thermophilous deciduous stand) - Common. Grasslands, forests, forest openings and forest roads. $600-1900 \mathrm{~m}$.

* Rosa corymbifera Borkh. - P caesp, EA - Chas. 1460 (22/6/2011, 22.20341 E, $41.11227 \mathrm{~N}, 880 \mathrm{~m}$, thermophilous deciduous stand), Chas. 1895 (24/5/2012, $22.20312 \mathrm{E}, 41.10686 \mathrm{~N}, 720 \mathrm{~m}$, shrubland of thermophilous deciduous and Juniperus oxycedrus) - Sporadic. Thermophilous deciduous stands and oak forests, of lowland areas.

*Rosa dumalis Bechst. - P caesp, EA - Chas. 139 (6/8/2009, 22.19142 E, 41.14685 N, $1560 \mathrm{~m}$, opening in beech stand with Vaccinium myrtillus and Juniperus communis subsp. nana), Chas. 1737 (19/10/2011, 22.22488 E, 41.13467 N, 1560 m, margins of Ostrya stand on stony ground), Chas. 2057 (21/6/2012, 22.22168 E, $41.14282 \mathrm{~N}$, $1630 \mathrm{~m}$, shrubland of Juniperus communis subsp. nana at treeline) - Sporadic. Shrublands at and above treeline.

*Rosa heckeliana Tratt. - P caesp, BI - Chas. 2061 (21/6/2012, 22.224 E, 41.14511 N, $1750 \mathrm{~m}$, subalpine shrubland), Chas. 2305 (18/6/2013, $22.23447 \mathrm{E}, 41.13707 \mathrm{~N}$, $1610 \mathrm{~m}$, shrubland of Juniperus communis subsp. nana), Chas. 2456 (14/7/2014, 22.23414 E, 41.14472 N, 1960 m, subalpine shrubland) - Sporadic. Shrublands at and above treeline. 
*Rosa pendulina L. - P caesp, Eu - Chas. 1588 (22/7/2011, 22.17377 E, 41.15529 N, 1910 m), Chas. 2059 (21/6/2012, 22.22203 E, 41.14301 N, 1650 m) - Common. In shrublands above treeline, rarely at lower altitudes.

Rosa pulverulenta M. Bieb. - P caesp, ME - Chas. 268 (28/8/2009, 22.21319 E, $41.14404 \mathrm{~N}, 1760 \mathrm{~m}$, subalpine shrubland), Chas. 314 (10/9/2009, 22.25447 E, $41.13756 \mathrm{~N}, 1210 \mathrm{~m}$, forest road in beech stand), Chas. 2060 (21/6/2012, 22.22156 E, $41.143 \mathrm{~N}, 1640 \mathrm{~m}$, shrubland of Juniperus communis subsp. nana at treeline), MFG1: 397 - Common. Shrublands at and above treeline, rarely at lower altitudes.

Rosa spinosissima L. - P caesp, Pt - Chas. 2058 (21/6/2012, 22.21718 E, 41.14608 N, $1770 \mathrm{~m}$, road above treeline), Chas. 2310 (18/6/2013, $22.23321 \mathrm{E}, 41.1394 \mathrm{~N}, 1780$ m, subalpine shrubland), Chas. 2395 (20/7/2013, 22.23475 E, 41.14852 N, 1690 m, rocky opening in beech stand at treeline), Chasapis et al. 2019 - Sporadic. Shrublands at and above treeline.

*Rosa villosa L. - P caesp, EA - Chas. 1730 (4/10/2011, 22.22466 E, 41.12963 N, 1520 m, opening in mixed stand of beech with Acer hyrcanum) - Rare. Beech forests and margins of beech forests at treeline.

*Rubus caesius L. - P caesp, EA - Chas. 1079 (22/4/2011, 22.23569 E, 41.118 N, 810 $\mathrm{m}$, rivulet in thermophilous deciduous stand) - Rare.

*Rubus canescens DC. - P caesp, EA - Chas. 162 (13/8/2009, 22.18554 E, 41.12244 $\mathrm{N}, 950 \mathrm{~m}$, opening in beech stand), Chas. $184(13 / 8 / 2009,22.18661 \mathrm{E}, 41.12282 \mathrm{~N}$, $1000 \mathrm{~m}$, forest road in beech stand), Chas. 1459 (22/6/2011, 22.21729 E, 41.11346 $\mathrm{N}, 790 \mathrm{~m}$, thermophilous deciduous stand) - Common. In beech and oak forests, thermophilous deciduous stands and forest roads. $700-1700 \mathrm{~m}$.

*Rubus hirtus Waldst. \& Kit. - P caesp, EA - Chas. 24 (11/7/2009, 22.18 E, 41.12893 $\mathrm{N}, 1000 \mathrm{~m}$ ) - Common. Beech forests and forest roads. $1000-1700 \mathrm{~m}$.

*Rubus idaeus L. - P caesp, Bo - Chas. 881 (24/7/2010, 22.24496 E, 41.14552 N, 1610 $\mathrm{m}$, opening in beech stand at treeline) - Common. In beech forest openings and in shrublands at and above treeline.

*Rubus sanctus Schreb. - P caesp, MS - Chas. 1466 (27/6/2011, 22.20764 E, 41.11153 $\mathrm{N}, 810 \mathrm{~m}$, forest road in thermophilous deciduous stand), Chas. 2157 (6/7/2012, 22.25925 E, 41.1279 N, $810 \mathrm{~m}$, forest road in Quercus frainetto stand) - Common. In oak forests and forest roads. $600-1400 \mathrm{~m}$.

Sanguisorba minor Scop. subsp. minor - H scap, EA - Chas. 227 (24/8/2009, 22.21376 E, $41.12306 \mathrm{~N}, 1100 \mathrm{~m}$, forest road in mixed thermophilous deciduous and beech stand), Chas. 1235 (25/5/2011, $22.20407 \mathrm{E}, 41.1112 \mathrm{~N}, 830 \mathrm{~m}$, forest road in thermophilous deciduous stand), Voliotis 1983: 167 - Common. Grasslands and forest roads, of lowland areas.

*Sanguisorba officinalis L. - H scap, EA - Chas. 262 (28/8/2009, 22.21948 E, 41.1626 N, 1890 m, above treeline fen) - Rare.

*Sorbus aria (L.) Crantz subsp. aria - P caesp, ME - Chas. 402 (9/10/2009, 22.22865 E, $41.12284 \mathrm{~N}, 1240 \mathrm{~m}$, rocky place with sparse vegetation), Chas. 849 (26/6/2010, 22.21917 E, $41.1254 \mathrm{~N}, 1220 \mathrm{~m}$, open thermophilous deciduous shrubland on rocky slope), Chas. 2183 (11/7/2012, 22.19598 E, $41.11972 \mathrm{~N}, 1120 \mathrm{~m}$, opening in beech stand) - Sporadic. In rocky or stony calcareous places with sparse vegetation.

*Sorbus aucuparia L. subsp. aucuparia - P caesp/P scap, EA - Chas. 1216 (20/5/2011, $22.22847 \mathrm{E}, 41.12086 \mathrm{~N}, 1150 \mathrm{~m}$, thermophilous deciduous stand), Chas. 1696 (13/9/2011, 22.24583 E, $41.15328 \mathrm{~N}, 1720 \mathrm{~m}$, mixed beech - fir stand at treeline) - 
Sporadic. Oak, thermophilous deciduous and beech forests, on stony calcareous ground. $800-1750 \mathrm{~m}$.

*Sorbus graeca (Spach) Schauer - P caesp, EA - Chas. 1742 (19/10/2011, 22.22286 E, $41.13437 \mathrm{~N}, 1430 \mathrm{~m}$, Ostrya stand on rocky slope) - Sporadic. At rocky or stony calcareous places with sparse vegetation.

*Sorbus X thuringiaca (Ilse) Fritsch - P caesp, Eu - Chas. 244 (28/8/2009, 22.20709 E, $41.12728 \mathrm{~N}, 1260 \mathrm{~m}$, roadside in beech stand), Chas. $418(17 / 10 / 2009,22.24418$ E, $41.13461 \mathrm{~N}, 1250 \mathrm{~m}$, thermophilous deciduous stand on stony ground), Chas. 1702 (13/9/2011, $22.24595 \mathrm{E}, 41.15328 \mathrm{~N}, 1710 \mathrm{~m}$, mixed beech - fir stand on stony ground) - Sporadic. Beech forests, Ostrya stands and forest roads, on stony ground. $1250-1750 \mathrm{~m}$.

*Sorbus torminalis (L.) Crantz - P caesp/P scap, EA - Chas. 369 (20/9/2009, 22.18372 E, $41.12394 \mathrm{~N}, 940 \mathrm{~m}$, beech stand) - Sporadic. In beech and oak forests.

\section{RUBIACEAE}

Asperula aristata subsp. condensata (Boiss.) Ehrend. \& Krendl - H scap, Bk - Chas. $2104(30 / 6 / 2012,22.2277$ E, 41.15381 N, $1830 \mathrm{~m})$, Chas. $2369(15 / 7 / 2013$, 22.24621 E, $41.16148 \mathrm{~N}, 1940 \mathrm{~m})$, Chas. 2373 (15/7/2013, 22.2477 E, 41.16367 N, 1980 m), Chas. 2397 (25/7/2013, 22.20978 E, 41.15534 N, 2060 m), Chasapis et al. 2019 - Common. Subalpine grasslands on stony ground.

Asperula aristata subsp. nestia (Rech. f.) Ehrend. \& Krendl - H scap, Bk - Chas. 895 $(24 / 7 / 2010,22.2568 \mathrm{E}, 41.14849 \mathrm{~N}, 1560 \mathrm{~m}$, grassy opening in beech stand), Chas. 2191 (15/7/2012, 22.24 E, 41.15274 N, 1770 m, subalpine grassland), MFG2: 285, Schuler 2004: tab. 21 - Common. In grasslands, up to $1800 \mathrm{~m}$.

Asperula purpurea subsp. apiculata (Sibth. \& Sm.) Ehrend. - C suffr, Bk - Chas. 956 $(14 / 8 / 2010,22.22488$ E, $41.11261 \mathrm{~N}, 700 \mathrm{~m}$, open shrubland of Juniperus oxycedrus and thermophilous deciduous on rocky ground), Chas. 2349 (1/7/2013, 22.2279 E, $41.13319 \mathrm{~N}, 1660 \mathrm{~m}$, sparse grassland on rocky ground), MFG2: 300 Sporadic. Rocky calcareous places usually with sparse vegetation. $650-1700 \mathrm{~m}$.

*Asperula purpurea (L.) Ehrend. subsp. purpurea - C suffr, ME - Chas. 2155 (6/7/2012, 22.25119 E, 41.12634 N, 850 m, Quercus pubescens stand), Chas. 2354 $(1 / 7 / 2013,22.22296 \mathrm{E}, 41.13059 \mathrm{~N}, 1460 \mathrm{~m}$, open shrubland of thermophilous deciduous and Juniperus oxycedrus on rocky ground), Chas. 2376 (15/7/2013, $22.25828 \mathrm{E}, 41.16455 \mathrm{~N}, 1820 \mathrm{~m}$, subalpine sparse grassland on rocky ground) Sporadic. Rocky calcareous places usually with sparse vegetation. $700-1850 \mathrm{~m}$.

*Crucianella graeca Boiss. - T scap, Bk - Chas. 1410 (16/6/2011, 22.22684 E, $41.11507 \mathrm{~N}, 860 \mathrm{~m}$, phrygana), Chas. 2013 (13/6/2012, $22.22812 \mathrm{E}, 41.11712 \mathrm{~N}$, $980 \mathrm{~m}$, grassland) - Sporadic. In grasslands and phrygana, of lowland areas.

*Cruciata laevipes Opiz - H scap, EA - Chas. 489 (25/4/2010, 22.19832 E, 41.10823 N, $770 \mathrm{~m}$, grassland on damp ground), Chas. 740 (12/6/2010, 22.17455 E, 41.1537 $\mathrm{N}, 1870 \mathrm{~m}$, grassy opening in subalpine shrubland) - Sporadic. Grasslands on mesic to damp ground. $700-1900 \mathrm{~m}$.

*Cruciata pedemontana (Bellardi) Ehrend. - T scap, EA - Chas. 1985 (4/6/2012, 22.24446 E, $41.11822 \mathrm{~N}, 760 \mathrm{~m}$, thermophilous deciduous stand) - Sporadic. At shady places in forests of lowland areas.

*Galium album Mill. subsp. pycnotrichum (Heinr. Braun) Krendl - H scap, EA - Chas. 1409 (16/6/2011, 22.2256 E, 41.11253 N, 700 m, thermophilous deciduous stand) Sporadic. In different habitats of lowland areas. 
Galium anisophyllon subsp. plebeium (Boiss. \& Heldr.) Ehrend. - H scap, Bk - Chas. 2096 (25/6/2012, 22.20843 E, 41.14793 N, 2010 m, subalpine shrubland), Chas. $2314(22 / 6 / 2013,22.19862 \mathrm{E}, 41.15832 \mathrm{~N}, 1910 \mathrm{~m}$, schistose scree with sparse herbaceous vegetation), MFG2: 323 - Sporadic. Subalpine shrublands and grasslands, on stony ground.

*Galium aparine L. - T scap, EA - Chas. 621 (19/5/2010, 22.2155 E, 41.11597 N, 980 $\mathrm{m}$, thermophilous deciduous stand) - Common. In forests. $600-1500 \mathrm{~m}$.

*Galium asparagifolium Boiss. \& Heldr. - H scap, EM - Chas. 1523 (7/7/2011, $22.22498 \mathrm{E}, 41.11342 \mathrm{~N}, 720 \mathrm{~m}$, rocky place in opening with phrygana within thermophilous deciduous stand), Chas. 2034 (18/6/2012, 22.23207 E, $41.11542 \mathrm{~N}$, $810 \mathrm{~m}$, open shrubland of Juniperus oxycedrus and thermophilous deciduous) Sporadic. Open shrublands or phrygana on stony and rocky places, up to $1650 \mathrm{~m}$.

*Galium breviramosum Krendl - H scap, Bk - Chas. 55 (20/7/2009, 22.22228 E, $41.14724 \mathrm{~N}, 1790 \mathrm{~m})$, Chas. $767(12 / 6 / 2010,22.17566 \mathrm{E}, 41.15678 \mathrm{~N}, 1860 \mathrm{~m})$, Chas. 907 (1/8/2010, 22.2139 E, 41.16153 N, 2060 m), Chas. 908 (1/8/2010, 22.21559 E, 41.16083 N, 2040 m), Chas. 1577 (22/7/2011, 22.17496 E, 41.15614 N, 1860 m), Chas. 2066 (21/6/2012, 22.22352 E, 41.14528 N, 1760 m) - Common in shrublands and grassy shrubland openings, above treeline.

*Galium divaricatum Lam. - T scap, Me - Chas. 1352 (9/6/2011, 22.22068 E, 41.1093 N, $640 \mathrm{~m}$, dry grassland) - Sporadic. Dry grasslands and phrygana. $600-800 \mathrm{~m}$.

Galium hellenicum Krendl - H scap, Bk - Chas. 64 (20/7/2009, 22.21425 E, 41.14442 N, 1750 m, subalpine shrubland), MFG2: 314 - Rare.

*Galium intricatum Margot \& Reut. - T scap, Bk - Chas. 1353 (9/6/2011, 22.22068 E, $41.1093 \mathrm{~N}, 640 \mathrm{~m}$, dry grassland), Chas. 1882 (24/5/2012, 22.22724 E, $41.11336 \mathrm{~N}$, $760 \mathrm{~m}$, phrygana on rocky ground), Chas. 1923 (30/5/2012, $22.19704 \mathrm{E}, 41.10713$ N, $750 \mathrm{~m}$, open thermophilous deciduous - Juniperus oxycedrus shrubland), Chas. 2443 (28/6/2014, 22.2312 E, 41.11243 N, 690 m, dry grassland) - Common. Dry grasslands, open shrublands and phrygana. $600-900 \mathrm{~m}$.

Galium odoratum (L.) Scop. - H scap, EA - Chas. 93 (27/7/2009, 22.1844 E, 41.1335 $\mathrm{N}, 1190 \mathrm{~m}$ ), Schuler 2004: map 62 - Common. In beech stands, from $1000 \mathrm{~m}$ up to the treeline.

Galium oreophilum Krendl - H scap, Bk - Chas. 364 (15/9/2009, 22.25999 E, $41.16286 \mathrm{~N}, 1760 \mathrm{~m}$, subalpine sparse grassland on stony ground), Chas. 854 $(26 / 6 / 2010,22.22386$ E, $41.12826 \mathrm{~N}, 1440 \mathrm{~m}$, rocky slope with sparse thermophilous deciduous shrubland), Chas. 1991 (8/6/2012, 22.21881 E, 41.11693 N, 970 m, phrygana with Juniperus oxycedrus), MFG2: 315 - Common at stony and rocky places with sparse vegetation, above treeline. Occasionally descending to $900 \mathrm{~m}$, in grasslands and phrygana stony and rocky places.

*Galium pseudaristatum Schur - H scap, Bk - Chas. 46 (11/7/2009, 22.18 E, 41.12893 N, $1000 \mathrm{~m}$, beech stand), Chas. $96(27 / 7 / 2009,22.1844 \mathrm{E}, 41.1335 \mathrm{~N}, 1190 \mathrm{~m}$, beech stand), Chas. 1500 (2/7/2011, 22.27738 E, 41.14583 N, 980 m, Quercus frainetto stand), Chas. 2168 (6/7/2012, 355910 E, 4555690 N, 790 m, Quercus frainetto stand), Chas. 2170 (6/7/2012, 355990 E, 4556960 N, 1010 m, Quercus frainetto stand) - Common. In Quercus frainetto stands and beech stands at the lower parts of the beech forest zone.

*Galium rotundifolium L. - H scap, EA - Chas. 2228 (5/8/2012, 22.19394 E, 41.12906 $\mathrm{N}, 1430 \mathrm{~m}$, beech stand) - Rare. 
*Galium verticillatum Danthoine - T scap, ME - Chas. 1209 (20/5/2011, 22.22876 E, $41.11456 \mathrm{~N}, 820 \mathrm{~m}$, phrygana on rocky ground) - Sporadic. In phrygana and dry grasslands, on stony and rocky ground of lowland areas.

Galium verum L. subsp. verum - H scap, Pt - Chas. 1522 (7/7/2011, 22.22443 E, 41.11161 N, 680 m, irrigation ditch), Parent 2005: 219 - Sporadic. Roads, dry grasslands and shrublands. $600-900 \mathrm{~m}$.

Sherardia arvensis L. - T scap, EA - Chas. 1036 (8/4/2011, 22.19805 E, 41.10489 N, $670 \mathrm{~m}$, grassy opening in shrubland of Juniperus oxycedrus and thermophilous deciduous), Voliotis 1983: 167 - Sporadic. Dry grasslands and grassy openings of shrublands. $600-1000 \mathrm{~m}$.

\section{RUTACEAE}

*Dictamnus albus L. - H scap, EA - Chas. 1411 (16/6/2011, 22.2262 E, 41.1165 N, $900 \mathrm{~m})$ - Sporadic. Thermophilous deciduous stands, mainly on calcareous substrates.

Haplophyllum coronatum Spach - H scap, Bk - Chas. 513 (2/5/2010, 22.21203 E, $41.11196 \mathrm{~N}, 790 \mathrm{~m}$, road in thermophilous deciduous - Juniperus oxycedrus shrubland), Chas. 1208 (20/5/2011, 22.22829 E, 41.1141 N, 800 m, phrygana), Chas. 1869 (19/5/2012, $22.22109 \mathrm{E}, 41.11165 \mathrm{~N}, 690 \mathrm{~m}$, road in thermophilous deciduous - Juniperus oxycedrus shrubland), Chas. 2347 (1/7/2013, 22.22925 E, $41.13591 \mathrm{~N}, 1620 \mathrm{~m}$, sparse grassland on stony ground at treeline), Voliotis 1983: 167 - Sporadic. Roads and stony places with phrygana or sparse grassy vegetation. $600-1650 \mathrm{~m}$.

\section{SALICACEAE}

*Populus alba L. - P scap, EA - Chas. 387 (27/9/2009, 22.21237 E, 41.13016 N, 1320 $\mathrm{m}$, forest road in beech stand) - Rare. Streams and ditches by roadsides. $600-1350$ $\mathrm{m}$.

*Populus nigra L. - P scap, EA - Chas. 2233 (9/8/2012, 22.2035 E, 41.12371 N, 1200 $\mathrm{m}$, beech stand) - Rare.

Populus tremula L. - P scap, Ct - Chas. 3 (11/7/2009, 22.18 E, 41.12893 N, 1000 m), FH1: map 41 - Sporadic. Beech forests.

Salix alba L. - P scap, EA - Chas. 14 (11/7/2009, 22.19346 E, 41.11599 N, 950 m), Voliotis 1983: 167, FH1: map 32 - Sporadic. Streams and ditches by roadsides. 600 - $1200 \mathrm{~m}$.

Salix amplexicaulis Bory - P caesp, Me - Chas. 238 (28/8/2009, 22.2104 E, 41.12841 $\mathrm{N}, 1280 \mathrm{~m}$, ditch by roadside in beech stand), FH1: map 38 - Sporadic. Streams and ditches by roadsides. $700-1750 \mathrm{~m}$.

Salix caprea L. - P caesp/P scap, ES - Chas. 11 (11/7/2009, 22.18 E, 41.12893 N, 1000 $\mathrm{m}$, beech stand), FH1: map 35 - Common. Beech forests and forest roads, occasionally above treeline on stony ground. $1000-1900 \mathrm{~m}$.

Salix elaeagnos Scop. - P caesp/P scap, ME - Chas. 287 (10/9/2009, 22.25565 E, $41.12902 \mathrm{~N}, 900 \mathrm{~m}$, forest road in oak stand), FH1: map 36 - Sporadic. Streams and ditches by roadsides. $700-1500 \mathrm{~m}$.

*Salix X flueggeana Willd. - P caesp, Eu - Chas. 255 (28/8/2009, 22.21823 E, $41.14691 \mathrm{~N}, 1790 \mathrm{~m}$, above treeline spring by roadside), Chas. 2069 (21/6/2012, 22.21823 E, $41.14691 \mathrm{~N}, 1790 \mathrm{~m}$, above treeline spring by roadside) - Rare.

Salix triandra L. - P caesp, ES - FH1: map 33. 


\section{SANTALACEAE}

Arceuthobium oxycedri (DC.) MB. - P ep, MS - Chas. 368 (20/9/2009, 22.19869 E, $41.11202 \mathrm{~N}, 880 \mathrm{~m}$ ), Voliotis 1983: 169, FH1: map 112 - Common. Juniperus oxycedrus shrublands, of lowland areas.

*Comandra umbellata subsp. elegans (Spreng.) Piehl - C suffr, BA - Chas. 2039 (18/6/2012, 22.23578 E, 41.128 N, 1210 m, open thermophilous deciduous stand on stony ground) - Rare. Thermophilous deciduous stands and oak forests on stony ground.

*Osyris alba L. - P caesp, Me - Chas. 1906 (24/5/2012, 22.22666 E, 41.11281 N, 720 m, phrygana) - Sporadic. Phrygana and open Juniperus oxycedrus shrublands, on stony and rocky ground of lowland areas.

Thesium arvense Horv. - H scap, EA - MFG1: 54.

Thesium divaricatum Mert. \& W. D. J. Koch - H scap, ME - Chas. 1212 (20/5/2011, 22.22817 E, $41.1141 \mathrm{~N}, 800 \mathrm{~m}$, phrygana), Chas. 1415 (16/6/2011, 22.22585 E, $41.11649 \mathrm{~N}, 880 \mathrm{~m}$, opening thermophilous deciduous - Juniperus oxycedrus stand), FH1: map 101 - Sporadic. Phrygana and openings of thermophilous deciduous - Juniperus oxycedrus shrublands, on stony ground of lowland areas.

Thesium linophyllon L. subsp. linophyllon - H scap, Eu - Chas. 704 (2/6/2010, $22.20058 \mathrm{E}, 41.13439 \mathrm{~N}, 1690 \mathrm{~m}$, opening of Juniperus communis subsp. nana shrubland at treeline), Chas. 1157 (6/5/2011, $22.27314 \mathrm{E}, 41.14802 \mathrm{~N}, 1120 \mathrm{~m}$, opening in mixed beech - oak stand), MFG1: 56, FH1: map 99 - Common. Grasslands and openings of shrublands and forests, on stony ground. $1000-1900$ $\mathrm{m}$.

Thesium linophyllon subsp. montanum (Schrad.) Čelak. - H scap, Eu - Chas. 705 (2/6/2010, 22.20058 E, 41.13439 N, 1690 m, opening of Juniperus communis subsp. nana shrubland at treeline), Chas. 1323 (2/6/2011, 22.23123 E, $41.13774 \mathrm{~N}$, $1690 \mathrm{~m}$, subalpine grassland on stony ground), FH1: map 100 - Sporadic. Common. Grasslands and openings of shrublands and forests, on stony ground. $1300-1900 \mathrm{~m}$.

Thesium parnassi A. DC. - H scap, BI - Chas. 2103 (30/6/2012, 22.22591 E, 41.15397 N, 1820 m), MFG1: 55, FH1: map 97 - Sporadic. In subalpine grasslands.

\section{SAXIFRAGACEAE}

*Chrysosplenium alternifolium L. - H scap, ES - Chas. 1771 (19/4/2012, 22.21293 E, $41.13143 \mathrm{~N}, 1320 \mathrm{~m}$, streambank in beech stand) - Rare.

*Saxifraga adscendens L. subsp. adscendens - H bienn, AA - Chas. 1327 (2/6/2011, 22.22875 E, $41.15005 \mathrm{~N}, 1680 \mathrm{~m}$, rocky streambank above treeline) - Rare.

Saxifraga aizoides L. - H scap, AA - Chas. 269 (28/8/2009, 22.21911 E, 41.16296 N, $1890 \mathrm{~m}$, above treeline spring by roadside), Chas. 1621 (18/8/2011, $22.24872 \mathrm{E}$, $41.13414 \mathrm{~N}, 1750 \mathrm{~m}$, above treeline spring by roadside), Parent 2005: 205, MFG1: 369, FH2: 348/ map 1315 - Sporadic. Springs and streambanks above treeline.

Saxifraga bulbifera L. - H scap, ME - Chas. 480 (25/4/2010, 22.19922 E, 41.11005 N, $820 \mathrm{~m}$, thermophilous deciduous stand), Chas. $521(2 / 5 / 2010,22.21345 \mathrm{E}$, $41.12108 \mathrm{~N}, 1070 \mathrm{~m}$, rocky opening in thermophilous deciduous stand), Chas. 598 $(12 / 5 / 2010,22.24872 \mathrm{E}, 41.13414 \mathrm{~N}, 1180 \mathrm{~m}$, forest road in mixed oak - beech stand), Voliotis 1983: 167, FH2: map 1318 - Common. Open forests and forest openings, up to $1400 \mathrm{~m}$. 
*Saxifraga carpetana subsp. graeca (Boiss. \& Heldr.) D. A. Webb - H scap, Me Chas. 994 (28/3/2011, 22.1929 E, 41.11472 N, 870 m, thermophilous deciduous stand) - Rare.

Saxifraga exarata Vill. - H scap, ME - Chas. 1152 (6/5/2011, 22.25388 E, 41.15088 N, $1710 \mathrm{~m}$, steep rocky slope at treeline), Voliotis 1983: 167, MFG1: 370, FH2: 350/ map 1319 - Rare.

Saxifraga federici-augusti subsp. grisebachii (Degen \& Dörfl.) D. A. Webb - H scap, Bk - Chas. $586(12 / 5 / 2010,22.24717$ E, $41.14303 \mathrm{~N}, 1610 \mathrm{~m}$, sparse grassland on stony slope), Voliotis 1983: 168 (as S. porophylla subsp. grisebachii (Degen \& Dörfl.) Aldén \& Strid), MFG1: 379 (S. porophylla subsp. grisebachii (Degen \& Dörfl.) Aldén \& Strid), FH2: map 1309, Schuler 2004: tab. 21 - Common. Stony places with sparse herbaceous vegetation at and above treeline.

?Saxifraga porophylla Bertol. - H scap, BI - Voliotis 1983: 168 (as S. porophylla Bertol. subsp. porophylla).

Saxifraga paniculata Mill. - H ros, AA - Chas. 760 (12/6/2010, 22.18097 E, 41.15858 $\mathrm{N}, 1820 \mathrm{~m}$, rocky streambank above treeline), Chas. 1377 (9/6/2011, 22.18666 E, $41.15543 \mathrm{~N}, 1660 \mathrm{~m}$, rocky streambank above treeline), Voliotis 1983: 168, FH2: map 1314 - Sporadic. Stony and rocky places with sparse vegetation above treeline.

* Saxifraga rotundifolia subsp. chrysosplenifolia (Boiss.) D. A. Webb - H scap, Bk Chas. $502(25 / 4 / 2010,22.18587$ E, 41.11929 N, 910 m, streambank in mixed beech - thermophilous deciduous stand), Chas. 528 (2/5/2010, 22.19793 E, $41.11363 \mathrm{~N}$, $910 \mathrm{~m}$, streambank in mixed beech - thermophilous deciduous stand) - Sporadic. Streambanks and springs in beech forests at the lower parts of the beech forest zone.

Saxifraga rotundifolia L. subsp. rotundifolia - H scap, ME - Chas. 801 (12/6/2010, 22.18478 E, 41.14576 N, 1510 m, beech stand), Chas. $2072(21 / 6 / 2012,22.20959$ E, $41.14038 \mathrm{~N}, 1710 \mathrm{~m}$, beech stand at treeline), Chas. $2133(30 / 6 / 2012,22.21923$ E, $41.16296 \mathrm{~N}, 1890 \mathrm{~m}$, above treeline spring by roadside), Voliotis 1983: 168, FH2: map 1299 - Common in damp places, mainly stony streambanks in beech forests. Rare in streambanks above treeline.

Saxifraga scardica Griseb. - C pulv, Bk - Chas. 462 (17/4/2010, 22.218 E, 41.12025 $\mathrm{N}, 1150 \mathrm{~m}$, rocky slope with sparse herbaceous vegetation), Chas. 537 (8/5/2010, 22.23426 E, $41.15373 \mathrm{~N}, 1860 \mathrm{~m}$, rocky place above treeline), Voliotis 1983: 168, MFG1: 373, FH2: map 1303, Schuler 2004: tab. 21 - Common. Calcareous rocky and stony places with sparse vegetation above treeline, rarely at lower altitudes.

Saxifraga sempervivum K. Koch - C pulv, BA - Schuler 2005: tab. 21.

*Saxifraga tridactylites L. - T scap, EA - Chas. 471 (17/4/2010, 22.21942 E, 41.11621 $\mathrm{N}, 930 \mathrm{~m}$, phrygana) - Common. In grasslands, forest roads and phrygana. 600 $1400 \mathrm{~m}$.

\section{SCROPHULARIACEAE}

*Scrophularia canina L. subsp. canina - H scap, ME - Chas. 800 (12/6/2010, $22.18793 \mathrm{E}, 41.13977 \mathrm{~N}, 1390 \mathrm{~m}$, rocky roadside in beech stand), Chas. 2313 (22/6/2013, 22.20004 E, 41.15843 N, 1890 m, scree above treeline) - Rare. Stony siliceous ground, mainly schistose screes above treeline and roadsides in beech stands.

*Scrophularia laciniata Waldst. \& Kit. - H scap, Bk - Chas. 2094 (25/6/2012, 22.2069 E, 41.14746 N, 2010 m, subalpine grassland) - Rare. 
*Scrophularia nodosa L. - H scap, ES - Chas. 2277 (14/6/2013, 22.2655 E, 41.15313 N, 1230 m, small stream in beech forest) - Rare.

*Verbascum banaticum Schrad. - H bienn, Bk - Chas. 2043 (21/6/2012, 22.19349 E, $41.11509 \mathrm{~N}, 920 \mathrm{~m}$, roadside in thermophilous deciduous stand) - Sporadic. At forest roads and dry grasslands. $600-1000 \mathrm{~m}$.

*Verbascum dieckianum Borbás \& Degen - H bienn, Bk - Chas. 904 (1/8/2010, 22.21645 E, $41.11166 \mathrm{~N}, 730 \mathrm{~m}$, roadside in thermophilous deciduous - Juniperus oxycedrus shrubland), Chas. 1527 (12/7/2011, 22.22157 E, 41.11174 N, 690 m, road embankment in thermophilous deciduous - Juniperus oxycedrus shrubland), Chas. 1715 (24/9/2011, 22.23437 E, 41.10969 N, 630 m, dry grassland) - Sporadic. Roads and dry grasslands. $600-800 \mathrm{~m}$.

*Verbascum eriophorum Godr. - H bienn, Bk - Chas. 1 (11/7/2009, 22.18441 E, $41.12477 \mathrm{~N}, 1010 \mathrm{~m}$, roadside in beech stand), Chas. $236(24 / 8 / 2009,22.21376 \mathrm{E}$, $41.12306 \mathrm{~N}, 1100 \mathrm{~m}$, roadside in mixed beech - thermophilous deciduous stand) Sporadic. Stony roadsides in beech and oak forests.

*Verbascum longifolium Ten. - H scap, BI - Chas. 60 (20/7/2009, 22.22228 E, $41.14724 \mathrm{~N}, 1790 \mathrm{~m}$, subalpine grassland), Chas. 386 (27/9/2009, 22.20335 E, $41.13353 \mathrm{~N}, 1630 \mathrm{~m}$, forest road in beech stand), Chas. $2113(30 / 6 / 2012,22.22418$ E, 41.15169 N, $1770 \mathrm{~m}$, subalpine grassland) Common. Subalpine grasslands and roadsides of beech stands.

*Verbascum nigrum subsp. abietinum (Borbás) I. K. Ferguson - H scap, Bk - Chas. 32 $(11 / 7 / 2009,22.18 \mathrm{E}, 41.12893 \mathrm{~N}, 1000 \mathrm{~m}$, forest road in beech stand) - Common. Oak and beech forests and forest roads. $750-1500 \mathrm{~m}$.

*Verbascum phlomoides L. - H scap, EA - Chas. 317 (10/9/2009, 22.26185 E, 41.12866 N, 790 m, forest road in Quercus frainetto stand), Chas. 356 (15/9/2009, 22.25928 E, $41.16285 \mathrm{~N}, 1750 \mathrm{~m}$, subalpine grassland) - Sporadic. In grasslands and forest roads. $650-1800 \mathrm{~m}$.

Verbascum xanthophoeniceum Griseb. - H scap, BA - Chas. 660 (24/5/2010, 22.26465 E, $41.14447 \mathrm{~N}, 1170 \mathrm{~m}$, forest road in mixed oak - beech stand), Chas. 1109 (26/4/2011, 22.20094 E, $41.10836 \mathrm{~N}, 770 \mathrm{~m}$, grassy opening in Juniperus oxycedrus - thermophilous deciduous shrubland), Chas. 1221 (20/5/2011, 22.22724 E, 41.11336 N, 750 m, phrygana with Juniperus oxycedrus), Chas. 1810 (2/5/2012, $22.20656 \mathrm{E}, 41.11169 \mathrm{~N}, 830 \mathrm{~m}$, grassy opening by roadside in thermophilous deciduous stand), Voliotis 1983: 168 - Sporadic. Grasslands, open shrublands and roads. $600-1200 \mathrm{~m}$.

\section{SOLANACEAE}

Atropa bella-donna L. - H scap, ME - Chas. 87 (27/7/2009, 22.1844 E, 41.1335 N, $1190 \mathrm{~m}$, forest road in beech stand), Schuler 2004: map 41 - Sporadic. In beech forests and forest roads.

*Solanum dulcamara L. - P caesp, Pt - Chas. 1705 (18/9/2011, 22.17914 E, 41.13873 $\mathrm{N}, 1180 \mathrm{~m}$, streambank in beech stand) - Rare.

*Solanum nigrum L. - T scap, Co - Chas. 967 (26/9/2010, 22.27482 E, 41.13822 N, $890 \mathrm{~m}$, forest road in Quercus frainetto stand) - Sporadic. At forest roads and other disturbed sites, up to $900 \mathrm{~m}$.

\section{$\underline{\text { THYMELAEACEAE }}$}


Daphne laureola L. - P caesp, ME - Chas. 22 (11/7/2009, 22.18 E, 41.12893 N, 1000 m), Voliotis 1983: 168 - Sporadic. In beech stands.

*Daphne mezereum L. - P caesp, ES - Chas. 2194 (15/7/2012, 22.24253 E, 41.15638 $\mathrm{N}, 1650 \mathrm{~m}$, beech stand on stony ground below treeline) - Rare.

Daphne oleoides Schreb. - C frut, Me - Chas. 677 (24/5/2010, 22.2478 E, 41.15547 N, $1650 \mathrm{~m}$, grassland on stony ground at treeline), MFG1: 592 - Sporadic. On stony places with sparse vegetation of herbs or shrubs. $1400-2000 \mathrm{~m}$.

*Thymelaea passerina (L.) Coss. \& Germ. - T scap, EA - Chas. 1677 (10/9/2011, 22.26805 E, 41.15109 N, 1220 m, forest road in beech stand) - Rare.

\section{TILIACEAE}

*Tilia tomentosa Moench - P scap, EA - Chas. 1739 (19/10/2011, 22.22836 E, 41.1205 $\mathrm{N}, 110 \mathrm{~m}$, margins of thermophilous deciduous stand on stony ground) - Rare.

\section{ULMACEAE}

*Celtis australis L. - P scap, ME - Chas. 1354 (9/6/2011, 22.21812 E, 41.10692 N, 610 $\mathrm{m}$, roadside in dry grassland) - Rare. At forest roads and thermophilous deciduous stands.

*Ulmus glabra Huds. - P scap, EA - Chas. 811 (26/6/2010, 22.22406 E, 41.11638 N, $790 \mathrm{~m}$, thermophilous deciduous stand) - Rare.

*Ulmus minor Mill. subsp. minor - P caesp/P scap, EA - Chas. 1800 (29/4/2012, $22.21097 \mathrm{E}, 41.11167 \mathrm{~N}, 800 \mathrm{~m}$, thermophilous deciduous stand), Chas. 1827 (5/5/2012, 22.20709 E, $41.10999 \mathrm{~N}, 780 \mathrm{~m}$, Platanus thicket in grassland) Sporadic. Shrublands. $600-900 \mathrm{~m}$.

\section{URTICACEAE}

*Parietaria officinalis L. - H scap, EA - Chas. 953 (14/8/2010, 22.22487 E, 41.11288 N, 690 m, rocky streambank), Chas. 1727 (28/9/2011, 22.19177 E, 41.11272 N, 720 m, swampy place with alders) - Rare.

*Urtica dioica L. - H scap, Co - Chas. 905 (1/8/2010, 22.21559 E, 41.16083 N, 2040 $\mathrm{m}$, subalpine shrubland) - Common. At forest roads, grasslands and shrublands above treeline, open beech stands and Pteridium thickets. From low to high altitudes, mainly on gentle slopes where live-stock is concentrated.

\section{VALERIANACEAE}

*Valeriana italica Lam. - H scap, EM - Chas. 622 (19/5/2010, 22.2155 E, 41.11597 N, 980 m), Chas. 1083 (22/4/2011, 22.23521 E, 41.11835 N, 830 m) - Rare. Open thermophilous deciduous stands on stony or rocky, calcareous ground.

Valeriana montana L. - H scap, Eu - Chas. 2195 (15/7/2012, 22.24253 E, 41.15638 N, $1640 \mathrm{~m}$, beech stand at treeline), Chasapis et al. 2019 - Rare.

Valerianella coronata (L.) DC. - T scap, EA - Chas. 1022 (8/4/2011, 22.22872 E, $41.11131 \mathrm{~N}, 660 \mathrm{~m}$, grassland), Chas. 1100 (26/4/2011, 22.19913 E, $41.10491 \mathrm{~N}$, $670 \mathrm{~m}$, grassy opening in thermophilous deciduous - Juniperus oxycedrus shrubland), Voliotis 1983: 168 - Sporadic. Grasslands and shrubland openings, up to $900 \mathrm{~m}$.

*Valerianella locusta (L.) Laterr. - T scap, EA - Chas. 1023 (8/4/2011, 22.19878 E, $41.10436 \mathrm{~N}, 650 \mathrm{~m}$, grassy opening in thermophilous deciduous - Juniperus oxycedrus shrubland) - Sporadic. Grasslands and shrubland openings, up to $900 \mathrm{~m}$. 
Valerianella rimosa Bast. - T scap, EA - Voliotis 1983: 168.

*Valerianella turgida (Steven) Betcke - T scap, MS - Chas. 1024 (8/4/2011, 22.19856 E, $41.10391 \mathrm{~N}, 640 \mathrm{~m}$, grassy opening in thermophilous deciduous - Juniperus oxycedrus shrubland) - Sporadic. Grasslands and shrubland openings, up to $900 \mathrm{~m}$.

\section{VERBENACEAE}

*Verbena officinalis L. - H scap, Ct - Chas. 947 (14/8/2010, 22.1935 E, 41.10581 N, $640 \mathrm{~m}$, disturbed site in Platanus stand) - Rare.

\section{VERONICACEAE}

*Chaenorhinum minus (L.) Lange - T scap, ME - Chas. 198 (24/8/2009, 22.21379 E, $41.12189 \mathrm{~N}, 1100 \mathrm{~m}$, forest road in mixed beech - thermophilous deciduous stand), Chas. $1681(10 / 9 / 2011,22.26805 \mathrm{E}, 41.15109 \mathrm{~N}, 1220 \mathrm{~m}$, forest road in beech stand) - Sporadic. Forest roads and grasslands. $600-1300 \mathrm{~m}$.

Digitalis grandiflora Mill. - H scap, Eu - Chas. 42 (11/7/2009, 22.18 E, 41.12893 N, $1000 \mathrm{~m}$, beech stand), Chas. 1507 (2/7/2011, 22.27636 E, $41.14955 \mathrm{~N}, 1030 \mathrm{~m}$, Quercus frainetto stand), Parent 2005: 217 - Sporadic. Oak and beech stands. 800 $1300 \mathrm{~m}$.

Digitalis viridiflora Lindl. - H scap, Bk - Chas. 137 (6/8/2009, 22.19156 E, 41.14632 N, 1560 m, roadside in beech stand), Parent 2005: 217 - Sporadic. Forest roads and openings in beech forests.

*Linaria dalmatica (L.) Mill. - H scap, EM - Chas. 245 (28/8/2009, 22.21245 E, $41.12746 \mathrm{~N}, 1290 \mathrm{~m}$, roadside in beech stand), Chas. 1688 (10/9/2011, 22.26486 E, $41.16366 \mathrm{~N}, 1690 \mathrm{~m}$, grassland on stony ground at treeline) - Rare.

*Linaria genistifolia (L.) Mill. - H scap, EA - Chas. 1360 (9/6/2011, 22.2074 E, $41.11152 \mathrm{~N}, 820 \mathrm{~m}$, roadside in thermophilous deciduous stand), Chas. 1442 (22/6/2011, 22.22068 E, 41.10921 N, 640 m, dry grassland) - Sporadic. Roads and dry grasslands of lowland areas.

*Linaria pelisseriana (L.) Mill. - T scap, MS - Chas. 1925 (30/5/2012, 22.19745 E, $41.10743 \mathrm{~N}, 760 \mathrm{~m}$, open shrubland of Juniperus oxycedrus) - Sporadic. Dry grasslands, phrygana and open shrublands. $600-900 \mathrm{~m}$.

*Linaria simplex (Willd.) DC. - T scap, MS - Chas. 1178 (11/5/2011, 22.22418 E, 41.11187 N, 680 m, dry grassland), Chas. 1870 (19/5/2012, 22.20372 E, 41.11129 $\mathrm{N}, 840 \mathrm{~m}$, forest road in thermophilous deciduous stand) - Dry grasslands, forest roads, phrygana and open shrublands. $600-900 \mathrm{~m}$.

*Veronica acinifolia L. - T scap, ME - Chas. 2270 (10/6/2013, 22.1845 E, 41.14305 N, $1480 \mathrm{~m}$, damp place at forest road in beech stand) - Rare.

Veronica anagallis-aquatica L. - H scap/T scap, Co - Chas. 1595 (13/8/2011, 22.22706 E, 41.1112 N, $660 \mathrm{~m}$, stream in grassland), Voliotis 1983: 168 - Rare.

*Veronica arvensis L. - Tscap, EA - Chas. 1951 (30/5/2012, 22.19459 E, 41.10569 N, $660 \mathrm{~m}$, opening in thermophilous deciduous shrubland) - Sporadic. Grasslands and shrubland openings, at lowland areas.

*Veronica beccabunga L. - Hrept, EA - Chas. 793 (12/6/2010, 22.18758 E, 41.14382 $\mathrm{N}, 1440 \mathrm{~m}$, damp place by roadside in beech stand), Chas. $2214(31 / 7 / 2012$, 22.17664 E, 41.1559 N, 1800 m, above treeline spring) - Sporadic. Damp places by roadsides and springs. $700-1800 \mathrm{~m}$.

*Veronica chamaedrys L. subsp. chamaedrys - H scap, ES - Chas. 652 (24/5/2010, 22.24705 E, $41.15663 \mathrm{~N}, 1700 \mathrm{~m}$, subalpine grassland), Chas. 1866 (16/5/2012, 
22.23929 E, 41.15264 N, $1760 \mathrm{~m}$, subalpine grassland) - Common. Subalpine grasslands and shrublands and beech stands at the higher parts of the beech forest zone. Up to $2100 \mathrm{~m}$.

*Veronica dillenii Crantz - T scap, EA - Chas. 1947 (30/5/2012, 22.19495 E, 41.10944 $\mathrm{N}, 760 \mathrm{~m}$, opening in thermophilous deciduous stand) - Sporadic. Grasslands and openings of forests and shrublands. On stony ground between 700 and $1800 \mathrm{~m}$.

*Veronica hederifolia L. - T scap, EA - Chas. 2239 (11/4/2013, 22.24863 E, 41.11504 $\mathrm{N}, 700 \mathrm{~m}$, grassy opening in thermophilous deciduous stand) - Sporadic. Grasslands, shrublands and forests, up to $1000 \mathrm{~m}$.

Veronica jacquinii Baumg. - H scap, EA - Chas. 573 (12/5/2010, 22.25085 E, $41.13039 \mathrm{~N}, 950 \mathrm{~m}$, grassland within oak forest), MFG2: 221 - Sporadic. Grasslands, shrublands and forests, up to $1800 \mathrm{~m}$.

*Veronica officinalis L. - H rept, EA - Chas. 118 (6/8/2009, 22.18389 E, 41.15205 N, $1590 \mathrm{~m}$, roadside in beech stand), Chas. 1684 (10/9/2011, 22.26368 E, $41.15868 \mathrm{~N}$, $1460 \mathrm{~m}$, beech stand) - Sporadic. Beech forests, on stony ground.

Veronica orsiniana Ten. subsp. orsiniana - C suffr/H caesp, ME - Chas. 651 $(24 / 5 / 2010,22.24705 \mathrm{E}, 41.15663 \mathrm{~N}, 1700 \mathrm{~m}$, subalpine grassland), Chas. 1365 (9/6/2011, 22.19703 E, $41.15108 \mathrm{~N}, 1720 \mathrm{~m}$, grassy opening in subalpine shrubland), MFG2: 220 - Common. Grasslands and shrubland openings. 1600 $2180 \mathrm{~m}$.

*Veronica persica Poir. - T scap, [W-As.] - Chas. 1760 (27/3/2012, 22.22671 E, $41.11092 \mathrm{~N}, 660 \mathrm{~m}$, grassland) - Rare. Grasslands and roads, up to $750 \mathrm{~m}$.

*Veronica polita Fr. - T scap, EA - Chas. 1008 (3/4/2011, 22.2099 E, 41.11156 N, 800 $\mathrm{m}$, roadside at margins of Pteridium thicket and thermophilous deciduous stand) Rare.

*Veronica serpyllifolia subsp. humifusa (Dicks.) Syme - T rept, AA - Chas. 1366 (9/6/2011, 22.1916 E, 41.14902 N, $1550 \mathrm{~m}$, roadside in beech stand), Chas. 2421 $(30 / 7 / 2013,22.21 \mathrm{E}, 41.16471 \mathrm{~N}, 2040 \mathrm{~m}$, tall-herb vegetation in rivulet above treeline) - Rare.

*Veronica serpyllifolia L. subsp. serpyllifolia - T rept, Ct - Chas. 696 (2/6/2010, 22.19272 E, 41.12994 N, 1380 m, forest road in beech stand) - Rare.

*Veronica urticifolia Jacq. - H scap, Eu - Chas. 27 (11/7/2009, 22.18 E, 41.12893 N, $1000 \mathrm{~m}$ ) - Common. In beech forests, mainly at streambanks inside them.

*Veronica verna L. - T scap, ES - Chas. 2046 (21/6/2012, 22.22206 E, 41.14625 N, $1760 \mathrm{~m})$ - Sporadic. Subalpine grasslands on stony ground.

Veronica vindobonensis (M. A. Fisch.) M. A. Fisch. - H scap, Eu - Chas. 1781 $(22 / 4 / 2012,22.23907 \mathrm{E}, 41.12075 \mathrm{~N}, 870 \mathrm{~m}$, thermophilous deciduous stand), Chas. $2273(10 / 6 / 2013,22.17108 \mathrm{E}, 41.14554 \mathrm{~N}, 1540 \mathrm{~m}$, beech stand), Voliotis 1983: 168 - Common. Forests and shrublands. 600 - $1600 \mathrm{~m}$.

\section{VIOLACEAE}

*Viola aetolica Boiss. \& Heldr. - H scap, Bk - Chas. 447 (10/4/2010, 22.22918 E, $41.12537 \mathrm{~N}, 1320 \mathrm{~m}$, open thermophilous deciduous and Juniperus oxycedrus stand) - Rare.

*Viola alba Besser subsp. alba - H ros, EA - Chas. 1044 (11/4/2011, 22.21073 E, $41.11185 \mathrm{~N}, 800 \mathrm{~m}$, thermophilous deciduous stand), Chas. $1756(22 / 3 / 2012$, 22.25079 E, $41.13246 \mathrm{~N}, 1040 \mathrm{~m}$, oak forest), Chas. 2449 (6/7/2014, 22.18951 E, 
41.12557 N, $1200 \mathrm{~m}$, beech forest) - Common. Oak, thermophilous deciduous and beech forests. $600-1600 \mathrm{~m}$.

?Viola allchariensis Beck subsp. allchariensis - Hscap, Bk - Voliotis 1983: 168.

*Viola arvensis Murray - T scap, EA - Chas. 1084 (22/4/2011, 22.23431 E, 41.1205 N, $960 \mathrm{~m}$, grassy opening in thermophilous deciduous - Juniperus oxycedrus stand on stony ground), Chas. 1792 (25/4/2012, 22.27738 E, 41.14511 N, 960 m, roadside in Quercus frainetto stand) - Sporadic. Grasslands and roads of lowland areas.

*Viola doerfleri Degen X frondosa (Velen.) Hayek - H scap - Chas. 542 (8/5/2010, 22.23426 E, $41.15355 \mathrm{~N}, 1850 \mathrm{~m})$, Chas. 766 (12/6/2010, 22.17317 E, $41.15206 \mathrm{~N}$, $1850 \mathrm{~m})$, Chas. 1841 (11/5/2012, 22.17185 E, 41.15213 N, $1930 \mathrm{~m})$ - Sporadic. Grasslands above treeline on stony ground.

*Viola eximia Formànek X frondosa (Velen.) Hayek - H scap - Chas. 1816 (2/5/2012, $22.21439 \mathrm{E}, 41.14802 \mathrm{~N}, 1920 \mathrm{~m}$, open subalpine shrubland), Chas. 1822 (3/6/2012, 22.22315 E, 41.14591 N, $1780 \mathrm{~m}$, open subalpine shrubland), Chas. $1832(11 / 5 / 2012,22.19441$ E, $41.15519 \mathrm{~N}, 1770 \mathrm{~m}$, subalpine grassland) Sporadic. Grasslands and shrublands above treeline.

*Viola eximia Formànek - H scap, Bk - Chas. 425 (31/10/2009, 22.21109 E, 41.15536 $\mathrm{N}, 2060 \mathrm{~m}$, subalpine grassland), Chas. 541 (8/5/2010, $22.24473 \mathrm{E}, 41.15876 \mathrm{~N}$, $1820 \mathrm{~m}$, subalpine grassland), Chas. 1014 (3/4/2011, $22.22817 \mathrm{E}, 41.13653 \mathrm{~N}, 1670$ $\mathrm{m}$, rocky slope with sparse vegetation), Chas. 1015 (3/4/2011, 22.21787 E, $41.12529 \mathrm{~N}, 1160 \mathrm{~m}$, open thermophilous deciduous shrubland on rocky slope), Chas. $1055(16 / 4 / 2011,22.24369 \mathrm{E}, 41.14397 \mathrm{~N}, 1650 \mathrm{~m}$, grassland at treeline), Chas. 1154 (6/5/2011, 22.25281 E, $41.15077 \mathrm{~N}, 1740 \mathrm{~m}$, open grassland on stony ground at treeline), Chas. $1789(25 / 4 / 2012,22.25292 \mathrm{E}, 41.16005 \mathrm{~N}, 1720 \mathrm{~m}$, open grassland on stony ground above treeline), Chas. 1847 (16/5/2012, 22.24159 E, $41.15127 \mathrm{~N}, 1750 \mathrm{~m}$, open grassland on stony ground above treeline) - Common. Grasslands and shrublands, usually on stony calcareous ground, at and above treeline. Rarely appears at lowland areas.

*Viola frondosa (Velen.) Hayek - H scap, Bk - Chas. 446 (10/4/2010, 22.22918 E, $41.12537 \mathrm{~N}, 1320 \mathrm{~m}$, open thermophilous deciduous - Juniperus oxycedrus stand), Chas. 543 (8/5/2010, 22.22281 E, 41.14959 N, 1750 m, open subalpine shrubland), Chas. $986(23 / 3 / 2011,22.22774 \mathrm{E}, 41.1258 \mathrm{~N}, 1370 \mathrm{~m}$, grassy opening in thermophilous deciduous - Juniperus oxycedrus shrubland), Chas. 1155 (6/5/2011, $22.25258 \mathrm{E}, 41.1505 \mathrm{~N}, 1720 \mathrm{~m}$, sparse grassland on stony ground at treeline), Chas. $1823(2 / 5 / 2012,22.22368 \mathrm{E}, 41.14375 \mathrm{~N}, 1700 \mathrm{~m}$, subalpine shrubland), Chas. 1839 (11/5/2012, 22.19358 E, 41.15499 N, $1760 \mathrm{~m}$, subalpine shrubland), Chas. 1849 (16/5/2012, 22.22262 E, 41.14788 N, 1790 m, subalpine grassland) Common. Grasslands and shrublands. $1200-1900 \mathrm{~m}$.

Viola grisebachiana Vis. s.lat. - H scap, Bk - Chas. 1547b (12/7/2011, 22.22881 E, 41.15324 N, $1860 \mathrm{~m}$, subalpine grassland), Chasapis et al. 2019 - Rare.

*Viola kitaibeliana Schult. - Tscap, ME - Chas. 472 (17/4/2010, 22.21942 E, 41.11621 N, 930 m, phrygana with Juniperus oxycedrus) - Common. Dry grasslands, phrygana and forest roads, of lowland areas.

*Viola macedonica Boiss. \& Heldr. - T scap, Bk - Chas. 338 (15/9/2009, 22.25928 E, $41.16285 \mathrm{~N}, 1750 \mathrm{~m}$, subalpine grassland), Chas. $555(8 / 5 / 2010,22.22281 \mathrm{E}$, 41.14959 N, $1750 \mathrm{~m}$, subalpine grassland), Chas. 1219 (20/5/2011, 22.22839 E, $41.12365 \mathrm{~N}, 1280 \mathrm{~m}$, grassland within Juniperus oxycedrus - thermophilous deciduous shrubland), Chas. 1379 (9/6/2011, 22.19228 E, 41.15894 N, $1860 \mathrm{~m}$, 
subalpine grassland), Chas. 2134 (30/6/2012, 22.22351 E, 41.15024 N, 1740 m tallherb vegetation above treeline) - Common. Grasslands above treeline and at midaltitudes.

Viola odorata L. - H ros, EA - Chas. 448 (10/4/2010, 22.22918 E, 41.12537 N, 1320 m, open thermophilous deciduous stand), Chas. 985 (23/3/2011, 22.21709 E, 41.11861 $\mathrm{N}, 1100 \mathrm{~m}$, thermophilous deciduous stand), Chas. 1005 (31/3/2011, $22.28359 \mathrm{E}$, $41.14007 \mathrm{~N}, 760 \mathrm{~m}$, beech stand in stream within Quercus frainetto forest), Chas. 1045 (11/4/2011, 22.20837 E, $41.11965 \mathrm{~N}, 1020 \mathrm{~m}$, mixed beech - thermophilous deciduous stand), Chas. $1840(11 / 5 / 2012,22.17185$ E, $41.15213 \mathrm{~N}, 1930 \mathrm{~m}$, grassland on steep slope above treeline), Voliotis 1983: 168 - Common. Forests and shrublands of low and mid-altitudes. Rarely above treeline.

*Viola reichenbachiana Jord. ex Boreau - H scap, EA - Chas. 216 (24/8/2009, 22.21662 E, $41.12743 \mathrm{~N}, 1140 \mathrm{~m}$, beech stand), Chas. 473 (17/4/2010, 22.21628 E, $41.12238 \mathrm{~N}, 1040 \mathrm{~m}$, beech stand), Chas. 991 (28/3/2011, 22.19054 E, $41.11414 \mathrm{~N}$, $790 \mathrm{~m}$, streambank in thermophilous deciduous forest) - Common. Beech stands and in the wettest places of oak and thermophilous deciduous forests.

Viola riviniana Reichenb. - H scap, Eu - Chas. 481 (25/4/2010, 22.19922 E, 41.11005 $\mathrm{N}, 820 \mathrm{~m}$, thermophilous deciduous stand), Chas. 1054 (16/4/2011, 22.26446 E, $41.13348 \mathrm{~N}, 880 \mathrm{~m}$, beech stand), Chas. 1848 (16/5/2012, 22.22063 E, $41.1465 \mathrm{~N}$, $1760 \mathrm{~m}$, subalpine shrubland), Voliotis 1983: 168, Tiniakou 1991: 156 - Common in beech stands and in the wettest places of oak and thermophilous deciduous forests. Rare in subalpine shrublands, up to $1900 \mathrm{~m}$.

Viola tricolor L. - T scap/H scap, ES - Voliotis 1983: 168.

\section{VITACEAE}

*Vitis vinifera L. - P lian, EA - Chas. 1909 (24/5/2012, 22.2256 E, 41.11253 N, 700 m, open thermophilous deciduous stand on stony ground) - Rare.

\section{ANGIOSPERMAE (MONOCOTYLEDONEAE)}

\section{ALLIACEAE}

Allium carinatum L. - G, Eu - Parent 2005: 225.

Allium cupani Raf. subsp. cupani - G bulb, Me - Chas. 406 (9/10/2009, 22.22813 E, $41.11995 \mathrm{~N}, 1110 \mathrm{~m}$, grassy opening in thermophilous deciduous stand), MFG2: 707 - Sporadic. In grasslands, phrygana and shrublands, on stony ground, up to $1700 \mathrm{~m}$.

*Allium flavum subsp. tauricum (Besser ex Rchb.) K. Richt. - G bulb, ME - Chas. 943 $(8 / 8 / 2010,22.22242 \mathrm{E}, 41.12428 \mathrm{~N}, 1090 \mathrm{~m}$, forest road in beech stand), Chas. $1603(13 / 8 / 2011,22.22542 \mathrm{E}, 41.1545 \mathrm{~N}, 1830 \mathrm{~m}$, subalpine grassland on stony ground), Chas. 2204 (27/7/2012, $22.26315 \mathrm{E}, 41.14273$ N, $1170 \mathrm{~m}$, Q. pubescens stand) - Common in subalpine grasslands. Sporadic at forest roads, oak and thermophilous deciduous forests.

Allium macedonicum Zahar. - G bulb, Gr - Chas. 1712 (24/9/2011, 22.23931 E, $41.12057 \mathrm{~N}, 870 \mathrm{~m}$ ), MFG2: 708 - Sporadic. In thermophilous deciduous stands, on calcareous substrates.

Allium melanantherum Pančić - G bulb, Bk - Chas. 1624 (18/8/2011, 22.22839 E, $41.14599 \mathrm{~N}, 1890 \mathrm{~m}$, sparse, subalpine grassland on stony ground), Chasapis et al. 2019 - Rare. 
Allium moschatum L. - G bulb, Eu - Chas. 942 (8/8/2010, 22.22255 E, 41.12374 N, $1070 \mathrm{~m}$, stony place in thermophilous deciduous stand), MFG2: 705 - Rare.

*Allium paniculatum L. - G bulb, ME - Chas. 173 (13/8/2009, 22.18554 E, 41.12244 $\mathrm{N}, 950 \mathrm{~m}$, grassy opening in beech stand) - Rare.

*Allium sphaerocephalon L. subsp. sphaerocephalon - G bulb, ME - Chas. 894 $(24 / 7 / 2010,22.2568 \mathrm{E}, 41.14849 \mathrm{~N}, 1560 \mathrm{~m}$, grassland in beech stand), Chas. 1521 (7/7/2011, 22.22452 E, 41.11269 N, $700 \mathrm{~m}$, phrygana on stony ground) - Common. In phrygana and sparse grasslands on stony places. Up to $1700 \mathrm{~m}$.

Allium ursinum L. - G bulb, EA - Chas. 456 (17/4/2010, 22.2207 E, 41.11731 N, 860 $\mathrm{m}$, streambank in mixed beech and thermophilous deciduous stand), Voliotis 1983: 169, Schuler 2004: map 50 - Sporadic. At damp places in beech forests, where forms a thick herb layer.

\section{ANTHERICACEAE}

Anthericum liliago L. - G bulb, - Chas. 634 (19/5/2010, 22.21658 E, 41.11554 N, 920 $\mathrm{m}$, phrygana on stony ground), Chas. 1197 (20/5/2011, 22.22559 E, $41.11262 \mathrm{~N}$, $700 \mathrm{~m}$, open thermophilous deciduous stand on stony ground), Voliotis 1983: 169 Sporadic. In phrygana and open thermophilous deciduous stands on stony calcareous ground.

\section{ARACEAE}

*Arum maculatum L. - G rhiz, ME - Chas. 903 (1/8/2010, 22.19764 E, 41.1202 N, $1130 \mathrm{~m}$, beech stand) - Rare. In mesic beech and oak forests.

\section{ASPARAGACEAE}

*Asparagus acutifolius L. - C frut, Me - Chas. 1189 (20/5/2011, 22.22451 E, 41.11314 $\mathrm{N}, 720 \mathrm{~m}$, thermophilous deciduous stand) - Sporadic. In thermophilous deciduous stands and oak forests.

*Asparagus tenuifolius Lam. - C frut, ME - Chas. 1065 (16/4/2011, 22.25131 E, 41.13075 N, 970 m, Quercus frainetto stand), Chas. 1076 (22/4/2011, 22.23912 E, $41.12336 \mathrm{~N}, 940 \mathrm{~m}$, thermophilous deciduous stand), Chas. 1713 (24/9/2011, 22.23719 E, $41.12432 \mathrm{~N}, 1040 \mathrm{~m}$, thermophilous deciduous stand) - Sporadic. In thermophilous deciduous stands and oak forests.

\section{ASPHODELACEAE}

*Asphodeline liburnica (Scop.) Rchb. - G rhiz, EM-Chas. 2031 (18/6/2012, 22.24198 E, $41.12791 \mathrm{~N}, 1060 \mathrm{~m})$ - Sporadic. Thermophilous deciduous stands on calcareous substrates.

*Asphodeline lutea (L.) Rchb. - G rhiz, ME- Chas. 461 (17/4/2010, 22.21942 E, $41.11621 \mathrm{~N}, 930 \mathrm{~m}$, phrygana with Juniperus oxycedrus) - Sporadic. Open thermophilous deciduous stands, grasslands and phrygana on stony ground, up to $1500 \mathrm{~m}$.

Asphodeline taurica (Pall.) Endl. - G rhiz, EA - Chas. 584 (12/5/2010, 22.24484 E, 41.1411 N, 1500 m, grassland on stony slope), Tan et al. 2006: 296 - Rare. Sparse grasslands at mid-altitudes on calcareous substrates.

\section{$\underline{\text { COLCHICACEAE }}$}


*Colchicum autumnale L. - G bulb, Eu - Chas. 300 (10/9/2009, 22.26008 E, 41.12827 $\mathrm{N}, 810 \mathrm{~m}$, oak forest) - Sporadic. In oak forests and forest openings.

*Colchicum doerfleri Halácsy - G bulb, Bk - Chas. 980 (23/3/2011, 22.22892 E, $41.11717 \mathrm{~N}, 970 \mathrm{~m}$, grassland), Chas. 1751 (18/3/2012, $22.25747 \mathrm{E}, 41.11427 \mathrm{~N}$, $680 \mathrm{~m}$, grassland) - Sporadic. In grasslands and phrygana, up to $1400 \mathrm{~m}$.

?Colchicum macedonicum Kosanin - G bulb, Bk - MFG2: 656.

\section{CONVALARIACEAE}

*Polygonatum odoratum (Mill.) Druce - G rhiz, ES - Chas. 494 (25/4/2010, 22.18587 E, 41.11929 N, $910 \mathrm{~m}$, beech stand) - Common in thermophilous deciduous stands on calcareous substrates. Sporadic in oak and beech forests.

\section{CYPERACEAE}

Blysmus compressus (L.) Link - G rhiz, ES - Chas. 276 (28/8/2009, 22.21948 E, $41.1626 \mathrm{~N}, 1890 \mathrm{~m})$, Chas. 2402 (25/7/2013, $22.22149 \mathrm{E}, 41.15876 \mathrm{~N}, 1850 \mathrm{~m})$, MFG2: 833, Schuler 2004: tab. 20 - Common. Above treeline fens.

Carex appropinquata Schumach. - H, ES - Parent 2005: 229.

*Carex caryophyllea Latourr. - H scap, ES - Chas. 734 (2/6/2010, 22.20024 E, $41.13807 \mathrm{~N}, 1790 \mathrm{~m}$, grassy opening in subalpine shrubland), Chas. 1063 (16/4/2011, 22.24779 E, 41.13331 N, 1130 m, oak forest), Chas. 1394 (9/6/2011, $22.18892 \mathrm{E}, 41.15573 \mathrm{~N}, 1690 \mathrm{~m}$, grassy opening in subalpine shrubland) Sporadic. In grasslands all-over the mountain.

*Carex davalliana Sm. - H caesp, Eu - Chas. 1386 (9/6/2011, 22.19093 E, 41.1563 N, 1730 m), Chas. 2111 (30/6/2012, 22.21971 E, 41.1627 N, 1880 m) - Common. Above treeline fens.

*Carex depauperata With. - H caesp, ME - Chas. 1487 (27/6/2011, 22.21629 E, 41.11795 N, $1080 \mathrm{~m})$, Chas. 1940 (30/5/2012, 22.19217 E, $41.11534 \mathrm{~N}, 880 \mathrm{~m})-$ Rare. In thermophilous deciduous stands.

*Carex digitata L. - H caesp, ES - Chas. 1486 (27/6/2011, 22.21629 E, 41.12556 N, 1120 m), Chas. 1801 (29/4/2012, 22.18364 E, 41.13971 N, 1380 m) - Common. In beech stands mainly on stony calcareous ground.

*Carex distachya Desf. - H caesp, Me - Chas. 1259 (25/5/2011, 22.21635 E, 41.11986 $\mathrm{N}, 1110 \mathrm{~m}$, open thermophilous deciduous stand) - Rare.

*Carex distans L. - H caesp, Eu - Chas. 1246 (25/5/2011, 22.20878 E, 41.12217 N, $1070 \mathrm{~m}$, opening in mixed beech - thermophilous deciduous stand), Chas. 1897 $(24 / 5 / 2012,22.20349 \mathrm{E}, 41.10651 \mathrm{~N}, 710 \mathrm{~m}$, grassy clearing in thermophilous deciduous stand) - Rare.

*Carex divulsa Stokes - H caesp, EA - Chas. 2084 (25/6/2012, 22.21219 E, 41.13241 $\mathrm{N}, 1360 \mathrm{~m}$, damp place by roadside in beech stand) - Rare.

Carex echinata Murray - H caesp, Ct - Chas. 1655 (23/8/2011, 22.2185 E, 41.16331 N, 1910 m), Chas. 2366 (10/7/2013, 22.21497 E, 41.1529 N, 1920 m), Schuler 2004: tab. 20 - Sporadic. Above treeline fens.

Carex ferruginea Scop. - G rhiz, Eu - Chas. 788 (12/6/2010, 22.20086 E, 41.15907 N, $1930 \mathrm{~m}$, streambank above treeline), Chas. 1339 (2/6/2011, 22.21895 E, 41.14233 N, 1610 m, swampy place at treeline), MFG2: 861, Schuler 2004: tab. $20-$ Common. Above treeline fens.

*Carex flacca Schreb. subsp. flacca - G rhiz, Eu - Chas. 532 (2/5/2010, 22.21113 E, $41.11897 \mathrm{~N}, 990 \mathrm{~m}$, damp place in mixed beech - thermophilous deciduous stand), 
Chas. $599(12 / 5 / 2010,22.24872 \mathrm{E}, 41.13414 \mathrm{~N}, 1170 \mathrm{~m}$, damp place in beech stand) - Sporadic. Damp places up to $1900 \mathrm{~m}$.

*Carex flacca subsp. serrulata (Spreng.) Greuter - G rhiz, MS - Chas. 1526 (7/7/2011, 22.20235 E, $41.10604 \mathrm{~N}, 690 \mathrm{~m}$, juniper and thermophilous deciduous stand) Common. Thermophilous deciduous stands and oak forests.

*Carex halleriana Asso - H caesp, EA - Chas. 1062 (16/4/2011, 22.25248 E, 41.14059 N, 1370 m, beech stand), Chas. 1092 (22/4/2011, 22.23912 E, 41.12336 N, 940 m, thermophilous deciduous stand) - Sporadic. Mainly in thermophilous deciduous stands and openings. Up to $1400 \mathrm{~m}$.

*Carex hirta L. - G rhiz, EA - Chas. 1890 (24/5/2012, 22.22718 E, 41.11111 N, 650 m, streambank in grassland) - Rare.

*Carex humilis Leyss. - H caesp, Pt - Chas. 1319 (2/6/2011, 22.22323 E, 41.13411 N, $1450 \mathrm{~m}$, mixed beech - Ostrya stand on stony ground), Chas. 1793 (25/4/2012, $22.2635 \mathrm{E}, 41.16111 \mathrm{~N}, 1610 \mathrm{~m}$, grassland on stony ground at treeline), Chas. 2374 (15/7/2013, 22.2477 E, 41.16367 N, 1980 m, subalpine grassland on stony ground) - Common. Subalpine shrublands and grasslands and thermophilous deciduous stands. On calcareous substrates between 1000 and $2050 \mathrm{~m}$.

Carex kitaibeliana Bech. - H caesp, ME - Chas. 570 (8/5/2010, 22.23426 E, 41.15355 $\mathrm{N}, 1850 \mathrm{~m})$, Chas. 571 (8/5/2010, $22.23426 \mathrm{E}, 41.15355 \mathrm{~N}, 1850 \mathrm{~m})$, Chas. 600 (12/5/2010, 22.24691 E, 41.14402 N, 1660 m), Chas. 787 (12/6/2010, 22.18097 E, 41.15858 N, 1820 m), MFG2: 859, Schuler 2004: tab. 21 - Common. Grasslands at and above treeline, mainly on stony calcareous ground.

*Carex leersii F.W. Schultz - H caesp, EA - Chas. 720 (2/6/2010, 22.2118 E, 41.11186 $\mathrm{N}, 790 \mathrm{~m}$, grassy clearing in thermophilous deciduous stand) - Rare.

*Carex leporina L. - H caesp, ES - Chas. 926 (1/8/2010, 22.21145 E, 41.15969 N, $2140 \mathrm{~m}$, subalpine grassland), Chas. 2052 (21/6/2012, 22.21223 E, $41.1397 \mathrm{~N}$, $1600 \mathrm{~m}$, ditch by roadside in beech stand) - Sporadic. Subalpine grasslands on damp ground and streambanks. Above $1500 \mathrm{~m}$.

*Carex liparocarpos Gaudin - G rhiz, EA - Chas. 644 (19/5/2010, 22.21465 E, $41.11641 \mathrm{~N}, 1010 \mathrm{~m}$, grassland on stony ground), Chas. $1041(8 / 4 / 2011,22.23524$ E, 41.11249 N, 690 m, phrygana), Chas. 1072 (22/4/2011, 22.23867 E, $41.12237 \mathrm{~N}$, $930 \mathrm{~m}$, grassy opening in thermophilous deciduous stand) - Common. Grasslands and phrygana, on stony ground, mainly below $1300 \mathrm{~m}$.

*Carex nigra (L.) Reichard - G rhiz, Ct - Chas. 2332 (26/6/2013, 22.22691 E, 41.15677 N, 1760 m, above treeline fen) - Rare.

*Carex otrubae Podp. - H caesp, EA - Chas. 1475 (27/6/2011, 22.21444 E, 41.12424 $\mathrm{N}, 1110 \mathrm{~m}$, damp place by roadside in beech stand) - Rare.

*Carex pallescens L. - H caesp, Ct - Chas. 2143 (30/6/2012, 22.22149 E, 41.15885 N, $1850 \mathrm{~m}$, above treeline fen), Chas. 2333 (26/6/2013, 22.23103 E, $41.15855 \mathrm{~N}, 1690$ $\mathrm{m}$, streambank above treeline with tall-herb vegetation) - Sporadic. Fens and other damp places above treeline.

*Carex panicea L. - G rhiz, ES - Chas. 1338 (2/6/2011, 22.21895 E, 41.14233 N, 1610 m, swampy place at treeline), Chas. $2051(21 / 6 / 2012,22.22022 \mathrm{E}, 41.14406 \mathrm{~N}$, $1660 \mathrm{~m}$, above treeline fen), Chas. 2112 (30/6/2012, 22.21971 E, 41.1627 N, 1890 $\mathrm{m}$, above treeline fen) - Sporadic in swamps and fens at and above treeline.

Carex paniculata L. - H caesp, EA - Chas. 566 (8/5/2010, 22.22587 E, 41.15973 N, $1750 \mathrm{~m}$, streambank above treeline), MFG2: 844, Schuler 2004: tab. 20 - Common. In fens and other damp places above treeline. 
*Carex pendula Huds. - H caesp, EA - Chas. 48 (11/7/2009, 22.18 E, 41.12893 N, $1000 \mathrm{~m}$, streambank in beech stand), Chas. 225 (24/8/2009, 22.21625 E, 41.12769 $\mathrm{N}, 1160 \mathrm{~m}$, streambank in beech stand) - Common. In damp sites of beech forests.

*Carex punctata Gaudin - H caesp, ME - Chas. 1953 (4/6/2012, 22.20426 E, 41.11733 N, 980 m, swamp in thermophilous deciduous stand) - Rare.

*Carex remota L. - H caesp, EA - Chas. 833 (26/6/2010, 22.22206 E, 41.12004 N, 900 $\mathrm{m}$, streambank in mixed beech - thermophilous deciduous stand) - Sporadic. In damp sites of beech forests.

*Carex sylvatica Huds. - H caesp, ES - Chas. 1485 (27/6/2011, 22.21629 E, 41.12556 $\mathrm{N}, 1120 \mathrm{~m})$ - Sporadic. In damp sites of beech forests.

*Carex viridula Michx. - H caesp, EA - Chas. 1954 (4/6/2012, 22.20426 E, 41.11733 N, $980 \mathrm{~m}$, swamp in thermophilous deciduous stand), Chas. 2364 (10/7/2013, 22.21497 E, 41.1529 N, 1920 m, above treeline swamp) - Rare.

*Eleocharis palustris (L.) R. Br. - G rhiz, Co - Chas. 1971 (4/6/2012, 22.20426 E, 41.11733 N, 980 m, swamp in thermophilous deciduous stand) - Rare.

*Eleocharis quinqueflora (Hartmann) O. Schwarz - G rhiz, Bo - Chas. 1653 (23/8/2011, 22.21672 E, 41.16337 N, 1950 m), Chas. 2363 (10/7/2013, 22.21497 E, $41.1529 \mathrm{~N}, 1930 \mathrm{~m})$ - Sporadic. Above treeline fens.

Eriophorum latifolium Hoppe - H caesp, EA - Chas. $272(28 / 8 / 2009,22.21827$ E, $41.1633 \mathrm{~N}, 1910 \mathrm{~m}$, above treeline fen), Chas. 1313 (2/6/2011, 22.21895 E, $41.14233 \mathrm{~N}, 1610 \mathrm{~m}$, swampy place at treeline), MFG2: 835 - Common. Swampy places at and above treeline.

*Schoenus nigricans L. - H caesp, Co - Chas. 1000 (28/3/2011, 22.20349 E, 41.10651 N, 710 m, damp site at road), Chas. 1768 (12/4/2012, 22.20278 E, 41.11064 N, 830 $\mathrm{m}$, damp site in Pteridium thicket) - Rare.

*Scirpoides holoschoenus (L.) Soják - G rhiz, Pt - Chas. 948 (14/8/2010, 22.19363 E, 41.10554 N, $640 \mathrm{~m}$, ditch by roadside) - Sporadic. Damp places by roadsides and grasslands, up to $1200 \mathrm{~m}$.

\section{DIOSCOREACEAE}

*Dioscorea communis (L.) Caddick \& Wilkin - G rad, ME - Chas. 939 (8/8/2010, 22.22337 E, 41.12456 N, $1160 \mathrm{~m}$, thermophilous deciduous stand) - Sporadic. In thermophilous deciduous forests and shrublands and oak forests.

\section{HYACINTHACEAE}

*Muscari botryoides (L.) Mill. - G bulb, ME - Chas. 1784 (22/4/2012, 22.23511 E, $41.13519 \mathrm{~N}, 1470 \mathrm{~m}$, grassland) - Rare. In grasslands of mid-altitudes and openings of beech forest.

*Muscari comosum (L.) Mill. - G bulb, ME - Chas. 1280 (28/5/2011, 22.20717 E, 41.11107 N, 800 m, Pteridium thicket) - Sporadic. In Pteridium thickets, grasslands and shrublands, up to $1400 \mathrm{~m}$.

Muscari neglectum Guss. ex Ten. - G bulb, EA - Chas. 450 (10/4/2010, 22.23015 E, $41.11602 \mathrm{~N}, 890 \mathrm{~m}$, phrygana on rocky ground), Chas. $1090(22 / 4 / 2011,22.24001$ E, $41.11671 \mathrm{~N}, 730 \mathrm{~m}$, grassy opening in thermophilous deciduous stand), Voliotis 1983: 169, MFG2: 699 - Common. In grasslands and stony open places all-over the mountain. 
Ornithogalum boucheanum (Kunth) Asch. - Gbulb, Eu - Chas. 1148 (6/5/2011, 22.27452 E, 41.14525 N, 1010 m), Voliotis 1983: 169 - Sporadic. In Pteridium thickets and grasslands of mesic ground. $1000-1650 \mathrm{~m}$.

*Ornithogalum divergens Boreau - G bulb, ME - Chas. 1010 (3/4/2011, 22.2099 E, $41.11156 \mathrm{~N}, 800 \mathrm{~m}$, grassy clearing in thermophilous deciduous stand), Chas. 2073 $(21 / 6 / 2012,22.22203 \mathrm{E}, 41.14751 \mathrm{~N}, 1790 \mathrm{~m}$, subalpine grassland) - Common in subalpine grasslands on mesic ground. Sporadic in grasslands at low and midaltitudes

Ornithogalum gussonei Ten. - G bulb, Me - Voliotis 1983: 169.

Ornithogalum kochii Parl. - G bulb, Me - Chas. 751 (12/6/2010, 22.19164 E, 41.15208 $\mathrm{N}, 1580 \mathrm{~m}$, opening in beech stand), Chas. 752 (12/6/2010, 22.19164 E, 41.15208 N, 1580 m, opening in beech stand), Chas. 1247 (25/5/2011, 22.19586 E, 41.11972 $\mathrm{N}, 1120 \mathrm{~m}$, road embankment in beech stand), Chas. $1248(25 / 5 / 2011,22.21392 \mathrm{E}$, $41.12153 \mathrm{~N}, 1080 \mathrm{~m}$, grassy opening $\mu \varepsilon$ stony ground in mixed thermophilous deciduous stand - beech), Chas. 1370 (9/6/2011, 22.19702 E, 41.15126 N, 1720 m, grassy opening in subalpine shrubland), Voliotis 1983: 169 (as O. orthophyllum Ten) - Common in stony places and grasslands above treeline. Sporadic in road embankments and grasslands or Pteridium thickets at low and mid-altitudes.

*Prospero autumnale (L.) Speta - G bulb, Me - Chas. 407 (9/10/2009, 22.22813 E, $41.11995 \mathrm{~N}, 1110 \mathrm{~m}$, grassy opening in thermophilous deciduous stand) - Sporadic. In openings of forests and shrublands, in grasslands and phrygana. Up to $1600 \mathrm{~m}$.

*Scilla subnivalis (Halácsy) Speta - G bulb, Gr - Chas. 438 (10/4/2010, 22.22918 E, $41.12537 \mathrm{~N}, 1320 \mathrm{~m}$, grassy opening in thermophilous deciduous stand), Chas. 551 (8/5/2010, 22.22594 E, $41.15721 \mathrm{~N}, 1760 \mathrm{~m}$, subalpine grassland) - Common. In grasslands and forests, mainly at and above treeline. $1000-2180 \mathrm{~m}$.

\section{IRIDACEAE}

*Crocus cancellatus Herb. - G bulb, BA - Chas. 1738 (19/10/2011, 22.22866 E, 41.12239 N, 1230 m, grassland) - Sporadic. Grasslands of lowland areas, openings of thermophilous deciduous and oak stands, phrygana. Up to $1400 \mathrm{~m}$.

*Crocus chrysanthus (Herb.) Herb. - G bulb, BA - Chas. 437 (10/4/2010, 22.22796 E, $41.13076 \mathrm{~N}, 1550 \mathrm{~m}$, montane grassland), Chas. 1753 (18/3/2012, 22.25933 E, $41.11592 \mathrm{~N}, 700 \mathrm{~m}$, grassland in thermophilous deciduous zone) - Sporadic. Grasslands and forest openings, up to the treeline.

*Crocus nivalis Bory \& Chaub. - G bulb, Bk - Chas. 1049 (11/4/2011, 22.20117 E, $41.13467 \mathrm{~N}, 1710 \mathrm{~m}$, grassy opening of low shrubland at treeline), Chas. 1056 $(16 / 4 / 2011,22.24487 \mathrm{E}, 41.14444 \mathrm{~N}, 1650 \mathrm{~m}$, grassy opening of low shrubland at treeline), Chas. 1755 (22/3/2012, 22.24784 E, $41.13151 \mathrm{~N}, 1050 \mathrm{~m}$, oak stand) Common. Grasslands and forests, mainly at and above treeline. $1000-2180 \mathrm{~m}$.

*Crocus pulchellus Herb. - G bulb, BA - Chas. 1744 (4/11/2011, 22.27045 E, $41.13257 \mathrm{~N}, 820 \mathrm{~m}$, open oak stand) - Common. Grasslands, forest openings and oak forests, ascending to the lower part of beech forest zone.

Crocus veluchensis Herb. - G bulb, Bk - Chas. 436 (10/4/2010, 22.22796 E, 41.13076 $\mathrm{N}, 1550 \mathrm{~m}$, grassy opening in shrubland), Chas. $550(8 / 5 / 2010,22.22594 \mathrm{E}$, $41.15721 \mathrm{~N}, 1750 \mathrm{~m}$, subalpine grassland), Chas. 1762 (2/4/2012, 22.20975 E, 41.12588 N, $1210 \mathrm{~m}$, beech stand), Voliotis 1983: 169 - Common. Grasslands and forests, mainly at and above treeline. $1000-2180 \mathrm{~m}$. 
*Gladiolus illyricus W. D. J. Koch - G bulb, ME - Chas. 850 (26/6/2010, 22.2291 E, $41.11952 \mathrm{~N}, 1080 \mathrm{~m}$, mixed shrubland of thermophilous deciduous with junipers) Sporadic. Open thermophilous deciduous stands.

*Iris reichenbachii Heuff. - G rhiz, Bk - Chas. 442 (10/4/2010, 22.23015 E, 41.11602 $\mathrm{N}, 890 \mathrm{~m}$, phrygana on stony ground) - Common. Stony ground with phrygana and open shrublands, at low altitudes.

*Romulea bulbocodium (L.) Sebast. \& Mauri - G buld, Me - Chas. 974 (13/3/2011, 22.20397 E, $41.11498 \mathrm{~N}, 960 \mathrm{~m}$, grassy opening in thermophilous deciduous stand), Chas. $1754(18 / 3 / 2012,22.25933 \mathrm{E}, 41.11592 \mathrm{~N}, 710 \mathrm{~m}$, grassland in thermophilous deciduous zone) - Common. Dry grasslands up to $1000 \mathrm{~m}$.

\section{JUNCACEAE}

*Juncus articulatus L. - G rhiz, Bo - Chas. 248 (28/8/2009, 22.21266 E, 41.13268 N, $1360 \mathrm{~m}$, damp place by roadside in beech stand), Chas. 1619 (18/8/2011, 22.21823 E, $41.14691 \mathrm{~N}, 1790 \mathrm{~m}$, ditch by roadside above treeline) - Common in damp places above treeline. Sporadic in damp places by roadsides of beech stands.

*Juncus bufonius L. - T caesp, Co - Chas. 1614 (18/8/2011, 22.2136 E, 41.13315 N, $1380 \mathrm{~m}$, damp place by roadside in beech stand) - Sporadic. Damp open places (mainly by roadsides), up to $1500 \mathrm{~m}$.

*Juncus compressus Jacq. - G rhiz/H caesp, EA - Chas. 1969 (4/6/2012, 22.20426 E, $41.11733 \mathrm{~N}, 980 \mathrm{~m}$, swamp in thermophilous deciduous stand) - Rare.

*Juncus conglomeratus L. - H caesp, Eu - Chas. 1643 (23/8/2011, 22.22001 E, $41.1473 \mathrm{~N}, 1800 \mathrm{~m}$, damp place by roadsides above treeline) - Rare.

*Juncus effusus L. - H caesp/G rhiz, Eu - Chas. 1599 (13/8/2011, 22.21737 E, 41.15257 N, $1850 \mathrm{~m}$, streambank above treeline) - Sporadic. Damp places in beech forests (mainly by roadsides) and above treeline.

*Juncus inflexus L. - H caesp/G rhiz, Pt - Chas. 232 (24/8/2009, 22.21635 E, $41.12851 \mathrm{~N}, 1170 \mathrm{~m}$, damp place in forest road in beech stand), Chas. 281 (28/8/2009, 22.21988 E, 41.16081 N, $1910 \mathrm{~m}$, streambank above treeline), Chas. $1638(23 / 8 / 2011,22.21361 \mathrm{E}, 41.13729 \mathrm{~N}, 1510 \mathrm{~m}$, damp place by roadside in beech stand) - Common. Damp places in beech stands (mainly by roadsides) and above treeline.

*Juncus tenuis Willd. - H caesp, [N-Am.] - Chas. 50 (11/7/2009, 22.18 E, 41.12893 N, $1000 \mathrm{~m}$, damp place by roadside in beech stand), Chas. $1556(17 / 7 / 2011,22.18828$ E, $41.13113 \mathrm{~N}, 1250 \mathrm{~m}$, damp place by roadside in beech stand) - Sporadic. Damp places in beech stands ascending to the lower part of the subalpine zone (mainly by roadsides).

*Juncus thomasii Ten. - G rhiz, Eu - Chas. 1567 (22/7/2011, 22.17448 E, 41.15613 N, $1890 \mathrm{~m}$, streambank above treeline) - Rare.

*Luzula campestris (L.) DC. - H caesp, EA - Chas. 568 (8/5/2010, 22.2295 E, 41.1533 N, 1850 m), Chas. 729 (2/6/2010, 22.20122 E, 41.13737 N, 1790 m), Chas. 1838 (11/5/2012, 22.19069 E, 41.15639 N, 1730 m) - Sporadic. Subalpine grasslands.

?Luzula congesta (Thuill.) Lej. - H caesp, Eu - Voliotis 1983: 169.

*Luzula forsteri (Sm.) DC. - H caesp, Me - Chas. 1061 (16/4/2011, 22.25345 E, $41.13988 \mathrm{~N}, 1290 \mathrm{~m})$ - Common. Thermophilous beech stands.

*Luzula luzulina (Vill.) Racib. - H caesp, Eu - Chas. 1308 (2/6/2011, 22.22255 E, 41.14599 N, 1760 m, open subalpine shrubland), Chas. 1797 (29/4/2012, 22.18451 E, $41.14692 \mathrm{~N}, 1520 \mathrm{~m}$, beech stand), Chas. 2274 (10/6/2013, 22.18463 E, 
$41.14684 \mathrm{~N}, 1520 \mathrm{~m}$, beech stand) - Common in mesic beech stands close to the treeline. Sporadic in subalpine shrublands and grasslands. Up to $1800 \mathrm{~m}$.

Luzula luzuloides (Lam.) Dandy \& Wilmott subsp. luzuloides - H caesp, Eu - Chas. 2263 (5/6/2013, 22.21437 E, 41.13127 N, 1280 m), Schuler 2004: map 61, Parent 2005: 227 - Common. In beech stands between 1200 and $1750 \mathrm{~m}$.

Luzula luzuloides subsp. rubella (Hoppe ex Mert. \& W. D. J. Koch) Holub - H caesp, $\mathrm{Eu}$ - Chas. $134(6 / 8 / 2009,22.18389 \mathrm{E}, 41.15205 \mathrm{~N}, 1600 \mathrm{~m}$, forest road in beech stand), Chas. $233(24 / 8 / 2009,22.21376 \mathrm{E}, 41.12306 \mathrm{~N}, 1100 \mathrm{~m}$, forest road in beech stand), Chas. 727 (2/6/2010, 22.19463 E, 41.12997 N, 1470 m, open beech stand), Chas. $789(12 / 6 / 2010,22.17434 \mathrm{E}, 41.15442 \mathrm{~N}, 1890 \mathrm{~m}$, subalpine shrubland), Chas. 1337 (2/6/2011, 22.21825 E, 41.14178 N, $1590 \mathrm{~m}$, shrubland at treeline), Parent 2005: 227 (as L. luzuloides subsp. cuprina) - Common. Beech forests and subalpine shrublands. Up to $2000 \mathrm{~m}$.

*Luzula multiflora (Ehrh.) Lej. - H caesp, Bo - Chas. 1073 (22/4/2011, 22.23805 E, $41.12326 \mathrm{~N}, 980 \mathrm{~m}$, thermophilous deciduous stand), Chas. $1798(29 / 4 / 2012$, 22.20682 E, $41.11097 \mathrm{~N}, 800 \mathrm{~m}$, Pteridium thicket in thermophilous deciduous zone) - Sporadic. Thermophilous deciduous and oak stands and stand openings.

Luzula spicata subsp. pindica (Hausskn.) Gamisans - H caesp, BI - Chas. 786 $(12 / 6 / 2010,22.17528 \mathrm{E}, 41.15759 \mathrm{~N}, 1890 \mathrm{~m}$, grassy opening in subalpine shrubland), Chas. 1385 (9/6/2011, 22.19879 E, 41.16084 N, 2060 m, subalpine grassland), MFG2: 741 - Common. Subalpine grasslands on siliceous substrates. Up to $2180 \mathrm{~m}$.

*Luzula sudetica (Willd.) Schult. - H caesp, AA - Chas. 2142 (30/6/2012, 22.22149 E, $41.15885 \mathrm{~N}, 1850 \mathrm{~m}$, above treeline fen), Chas. 2410 (25/7/2013, 22.2148 E, 41.1637 N, $1990 \mathrm{~m}$, spring above treeline) - Sporadic. Swampy places and damp grasslands above treeline.

Luzula sylvatica (Huds.) Gaudin - H caesp, Eu - Chas. 531 (2/5/2010, 22.1942 E, $41.12393 \mathrm{~N}, 1270 \mathrm{~m}$, beech stand), Chas. 567 (8/5/2010, 22.24072 E, $41.15698 \mathrm{~N}$, $1650 \mathrm{~m}$, beech stand at treeline), Parent 2005: 226 - Common in beech stands mainly on stony ground. Sporadic in subalpine shrublands, up to $1900 \mathrm{~m}$.

\section{LILIACEAE}

Fritillaria gussichiae (Degen \& Dörfl.) Rix - G bulb, Bk - Chas. 1094 (26/4/2011, $22.20054 \mathrm{E}, 41.10998 \mathrm{~N}, 830 \mathrm{~m}$, thermophilous deciduous stand), Chas. 1124 (1/5/2011, 22.22275 E, $41.11663 \mathrm{~N}, 810 \mathrm{~m}$, thermophilous deciduous stand), Chas. 1811 (2/5/2012, 22.20372 E, 41.1112 N, 830 m, Pteridium thicket), Voliotis 1983: 169 - Sporadic. In thermophilous deciduous forests and shrublands and in Pteridium thickets of lowland areas.

*Fritillaria montana Hoppe ex W. D. J. Koch - G bulb, Eu - Chas. 578 (12/5/2010, 22.24416 E, $41.14434 \mathrm{~N}, 1660 \mathrm{~m}$, grassland at treeline), Chas. 1125 (1/5/2011, $22.22133 \mathrm{E}, 41.11615 \mathrm{~N}, 870 \mathrm{~m}$, open thermophilous deciduous stand on stony slope) - Common. In grasslands, from lowlands up to the treeline, and open thermophilous deciduous stands. On stony calcareous ground, up to $1750 \mathrm{~m}$.

*Gagea lutea (L.) Ker Gawl. - G bulb, ES - Chas. 1174 (11/5/2011, 22.20311 E, 41.13362 N, $1630 \mathrm{~m}$, Pteridium thicket in beech stand), Chas. 1796 (29/4/2012, 22.17854 E, 41.14728 N, 1540 m, beech stand) - Common in Pteridium thickets at treeline. Sporadic in beech forests at treeline. 
Gagea minima (L.) Ker Gawl. - G bulb, ES - Chas. 549 (8/5/2010, 22.23068 E, $41.15368 \mathrm{~N}, 1840 \mathrm{~m}$, subalpine grassland), Chas. 1175 (11/5/2011, $22.20299 \mathrm{E}$, $41.13352 \mathrm{~N}, 1630 \mathrm{~m}$, Pteridium thicket in beech stand), Chas. 1795 (29/4/2012, $22.1801 \mathrm{E}, 41.14686 \mathrm{~N}, 1530 \mathrm{~m}$, Pteridium thicket in the beech forest zone), Chas. $1818(2 / 5 / 2012,22.21268 \mathrm{E}, 41.14502 \mathrm{~N}, 1800 \mathrm{~m}$, grassy opening in subalpine shrubland), Chas. 982 (23/3/2011, 22.22847 E, $41.12095 \mathrm{~N}, 1160 \mathrm{~m}$, grassy opening in thermophilous deciduous stand), MFG2: 663 - Common. Grasslands all-over the mountain, mainly above treeline.

*Gagea pratensis (Pers.) Dumort. - G bulb, Eu - Chas. 1761 (27/3/2012, 22.28494 E, 41.14792 N, 900 m, open oak stand), Chas. 1765 (5/4/2012, 22.2298 E, 41.12889 $\mathrm{N}, 1450 \mathrm{~m}$, grassy opening in shrubland) - Sporadic. Grasslands, shrublands and open forests, up to $1500 \mathrm{~m}$.

*Gagea pusilla (F. W. Schmidt) Sweet - G bulb, Eu - Chas. 981 (23/3/2011, 22.22847 E, $41.12095 \mathrm{~N}, 1160 \mathrm{~m}$, grassy opening in thermophilous deciduous stand), Chas. $1750(18 / 3 / 2012,22.23438 \mathrm{E}, 41.10906 \mathrm{~N}, 630 \mathrm{~m}$, grassland on stony ground), Chas. 1817 (2/5/2012, 22.21798 E, 41.14763 N, 1840 m, subalpine open shrubland) - Common. Grasslands and openings of forests and shrublands up to $1900 \mathrm{~m}$

*Gagea villosa (M. Bieb.) Sweet - G bulb, EA - Chas. 1757 (22/3/2012, 22.26677 E, 41.12748 N, 720 m, dry grassland) - Rare.

*Lilium albanicum Griseb. - G bulb, Bk - Chas. 763 (12/6/2010, 22.17808 E, $41.15124 \mathrm{~N}, 1700 \mathrm{~m}$, grassy opening in shrubland at treeline) - Sporadic. In grassland and shrubland openings above treeline, on siliceous substrates.

Lilium martagon L. - G bulb, EA - Chas. 820 (26/6/2010, 22.22138 E, 41.1185 N, 850 $\mathrm{m}$, streambank in mixed beech - thermophilous deciduous stand), Voliotis 1983: 169 - Common in mesic beech stands. Sporadic in oak forests and in Pteridium thickets at treeline.

\section{MELANTHIACEAE}

Veratrum lobelianum Bernh. - G rhiz, EA - Chas. 912 (1/8/2010, 22.21145 E, 41.15969 N, 2140 m), Voliotis 1983: 169, Schuler 2004: tab. 20 - Common. In subalpine mesic grasslands.

\section{ORCHIDACEAE}

Anacamptis coriophora subsp. fragrans (Pollini) R.M. Bateman, Pridgeon \& M. W. Chase - G bulb, EA - Chas. 807 (26/6/2010, 22.21299 E, 41.12044 N, $1050 \mathrm{~m}$, open thermophilous deciduous stand), Chas. 1359 (9/6/2011, 22.21965 E, 41.10793 N, 630 m, dry grassland), Voliotis 1983: 170 (as Orchis coriophora subsp. fragrans (Pollini) Sudre) - Sporadic. Grasslands and forest and shrubland openings, at lowland areas.

Anacamptis laxiflora (L.) R. M. Bateman, Pridgeon \& M. W. Chase subsp. laxiflora G bulb, MS - Chas. 1956 (4/6/2012, 41.11733 E, 22.20426 N, 980 m, swamp in thermophilous deciduous stand), Voliotis 1983: 170 (as Orchis laxiflora subsp. laxiflora Bech.) - Sporadic. Shrublands, phrygana and grasslands at low and midaltitudes.

Anacamptis morio subsp. caucasica (K. Koch) H. Kretzschmar, Eccarius \& H. Dietr. G bulb, EA - Chas. 1103 (26/4/2011, 22.19972 E, 41.10501 N, 670 m, grassy opening in juniper - thermophilous deciduous shrubland), Chas. 1182 (16/5/2011, 22.20097 E, $41.11133 \mathrm{~N}, 870 \mathrm{~m}$, grassy opening in thermophilous deciduous stand), 
Chas. $1241(25 / 5 / 2011,22.1961 \mathrm{E}, 41.1199 \mathrm{~N}, 1120 \mathrm{~m}$, grassy opening in mixed thermophilous deciduous - beech stand), Chas. 1298 (2/6/2011, 22.20162 E, $41.1226 \mathrm{~N}, 1180 \mathrm{~m}$, Juniperus oxycedrus thicket in beech stand), Chas. 1802 (2/5/2012, 22.21611 E, 41.11112 N, 720 m, phrygana with Juniperus oxycedrus), Chas. 1803 (2/5/2012, 22.21611 E, 41.11112 N, 720 m, phrygana with Juniperus oxycedrus), Voliotis 1983: 170 (as Orchis morio subsp. picta (Loisel.) Arcang.) Common. Grasslands and openings in forests and shrublands with phrygana or grassy vegetation. Up to $1300 \mathrm{~m}$.

*Anacamptis papilionacea (L.) R. M. Bateman, Pridgeon \& M. W. Chase - G bulb, MS - Chas. $1270(28 / 5 / 2011,22.18768 \mathrm{E}, 41.11842 \mathrm{~N}, 970 \mathrm{~m}$, forest road in mixed thermophilous deciduous - beech stand) - Sporadic. Grasslands, phrygana and roads of lowland areas.

*Anacamptis pyramidalis (L.) Rich. - G bulb, Eu - Chas. 790 (12/6/2010, 22.20359 E, $41.11137 \mathrm{~N}, 860 \mathrm{~m}$, forest road in thermophilous deciduous stand) - Sporadic. Grasslands, phrygana and forest roads of lowland areas.

*Cephalanthera damasonium (Mill.) Druce - G rhiz, ME - Chas. 2253 (1/6/2013, 22.2455 E, $41.13445 \mathrm{~N}, 1180 \mathrm{~m})$ - Rare. In beech stands on calcareous substrates.

Cephalanthera longifolia (L.) Fritsch - G rhiz, EA - Chas. 572 (12/5/2010, 22.25953 E, 41.13079 N, $910 \mathrm{~m}$, forest road in oak stand), Chas. 1097 (26/4/2011, 22.19908 E, $41.11103 \mathrm{~N}, 850 \mathrm{~m}$, thermophilous deciduous stand), Voliotis 1983: 169 Common in beech stands. Sporadic in oak forests and thermophilous deciduous stands. On calcareous substrates.

*Cephalanthera rubra (L.) Rich. - G rhiz, EA - Chas. 875 (4/7/2010, 22.1804 E, $41.13182 \mathrm{~N}, 1070 \mathrm{~m}$, beech stand) - Common in beech stands. Sporadic in oak forests and thermophilous deciduous stands. Mainly on calcareous substrates.

*Coeloglossum viride (L.) Hartm. - G bulb, Bo - obs. (15/7/2012, 22.23349 E, 41.15561 N, $1830 \mathrm{~m}$, subalpine dry grassland) - Rare.

*Corallorhiza trifida Châtel. - G rhiz, Bo - Chas. 757 (12/6/2010, 322.19138 E, $41.15289 \mathrm{~N}, 1620 \mathrm{~m})$ - Rare. In beech stands at treeline.

Dactylorhiza baumanniana J. Hölzinger \& Künkele - G bulb, Bk - Schuler 2004: tab. 20.

*Dactylorhiza cordigera (Fr.) Soó subsp. cordigera - G bulb, Bk - Chas. 1311 $(2 / 6 / 2011,22.21895 \mathrm{E}, 41.14233 \mathrm{~N}, 1610 \mathrm{~m}$, swamp at treeline), Chas. 1344 (9/6/2011, 22.19901 E, 41.15256 N, $1740 \mathrm{~m}$, streambank above treeline) Common. Swamps and streambanks at and above treeline.

?Dactylorhiza maculata (L.) Soó subsp. maculata - G bulb, ES - Voliotis 1983: 170.

?Dactylorhiza majalis (Rchb.) P. F. Hunt \& Summerh. subsp. majalis - G bulb, Eu Voliotis 1983: 170.

*Dactylorhiza saccifera (Brongn.) Soó - G bulb, Me - Chas. 4 (11/7/2009, 22.18 E, $41.12893 \mathrm{~N}, 1000 \mathrm{~m}$, streambank in beech stand), Chas. $37(11 / 7 / 2009,22.18 \mathrm{E}$, $41.12893 \mathrm{~N}, 1000 \mathrm{~m}$, streambank in beech stand), Chas. 73 (20/7/2009, 22.20846 E, $41.13838 \mathrm{~N}, 1690 \mathrm{~m}$, ditch by roadside in beech stand), Chas. $240(28 / 8 / 2009$, 22.20845 E, $41.13847 \mathrm{~N}, 1690 \mathrm{~m}$, ditch by roadside in beech stand) - Common in damp places in beech forests. Sporadic in damp places above treeline, up to 1850 $\mathrm{m}$.

*Dactylorhiza sambucina (L.) Soó - G bulb, Eu - Chas. 504 (2/5/2010, 22.21258 E, $41.12701 \mathrm{~N}, 1280 \mathrm{~m}$, grassy opening in beech stand), Chas. $515(2 / 5 / 2010$, $22.21113 \mathrm{E}, 41.11897 \mathrm{~N}, 990 \mathrm{~m}$, opening in mixed beech - thermophilous 
deciduous stand), Chas. 540 (8/5/2010, 22.22281 E, 41.14959 N, 1750 m, subalpine grassland) - Common in subalpine grasslands. Sporadic in openings of beech forests. $900-2100 \mathrm{~m}$.

*Epipactis atrorubens (Hoffm.) Besser - G rhiz, EA - Chas. 2358 (5/7/2013, 22.24921 E, 41.14712 N, $1500 \mathrm{~m}$, mixed beech - fir stand) - Sporadic. In beech forests.

*Epipactis helleborine (L.) Crantz - G rhiz, Pt - Chas. $38(11 / 7 / 2009,22.18$ E, $41.12893 \mathrm{~N}, 1000 \mathrm{~m}$, beech stand), Chas. 1508 (2/7/2011, 22.27636 E, 41.14955 N, $1030 \mathrm{~m}$, Quercus frainetto stand), Chas. 2167 (6/7/2012, 22.28543 E, $41.14712 \mathrm{~N}$, $870 \mathrm{~m}$, Quercus frainetto stand) - Sporadic. Oak and beech forests. Up to $1600 \mathrm{~m}$.

*Epipactis microphylla (Ehrh.) Sw. - G rhiz, EA - Chas. 815 (26/6/2010, 22.21914 E, $41.12224 \mathrm{~N}, 1020 \mathrm{~m}$, beech stand) - Rare.

Epipactis palustris (L.) Crantz - G rhiz, EA - Voliotis 1983: 170.

Gymnadenia conopsea (L.) R. Br. - G bulb, EA - Chas. 2056 (21/6/2012, 22.22046 E, $41.14388 \mathrm{~N}, 1660 \mathrm{~m}$, grassy opening in shrubland above treeline), Voliotis 1983: 170, Schuler 2004: tab. 20, Parent 2005: 231 - Sporadic. In grasslands above treeline, mainly on damp ground.

Gymnadenia frivaldii Hampe ex Griseb. - G bulb, Bk - Chas. 2138 (30/6/2012, 22.22149 E, $41.15885 \mathrm{~N}, 1850 \mathrm{~m}$, above treeline fen), MFG2: 869 - Sporadic. Swampy places above treeline.

*Himantoglossum jankae Somlyay, Kreutz \& Óvári - G bulb, BC - Chas. 1439 (22/6/2011, 22.21735 E, $41.11339 \mathrm{~N}, 780 \mathrm{~m}$, thermophilous deciduous stand) Rare.

*Limodorum abortivum (L.) Sw. - G bulb, Me - Chas. 1123 (1/5/2011, 22.22641 E, 41.11317 N, $750 \mathrm{~m}$, thermophilous deciduous scrub) - Rare.

Neotinea tridentata (L.) R. M. Bateman, Pridgeon \& M. W. Chase subsp. tridentata - G bulb, Me - Chas. 503 (2/5/2010, 22.22395 E, 41.11187 N, 680 m, phrygana), Chas. $1122(1 / 5 / 2011,22.22331 \mathrm{E}, 41.11781 \mathrm{~N}, 860 \mathrm{~m}$, opening with phrygana Juniperus oxycedrus within thermophilous deciduous stand), Voliotis 1983: 170 (as Orchis tridentata Scop. subsp. tridentata) - Common. Grasslands and phrygana, up to $1300 \mathrm{~m}$.

Neotinea ustulata (L.) R. M. Bateman, Pridgeon \& M. W. Chase - G bulb, Eu - Chas. $1322(2 / 6 / 2011,22.23955 \mathrm{E}, 41.14301 \mathrm{~N}, 1680 \mathrm{~m}$, grassland at treeline), Voliotis 1983: 170 (as Orchis ustulata L.) - Rare.

Neottia nidus-avis (L.) Rich. - G bulb, EA - Chas. 583 (12/5/2010, 22.24741 E, $41.13412 \mathrm{~N}, 1160 \mathrm{~m}$, beech - oak stand), Voliotis 1983: 170 - Common in beech stands. Sporadic in mesic oak forests and thermophilous deciduous stands.

Neottia ovata (L.) Bluff \& Fingerh. - G bulb, EA - Chas. 477 (25/4/2010, 22.19832 E, $41.10823 \mathrm{~N}, 770 \mathrm{~m}$, grassy clearing in thermophilous deciduous stand), Chas. 756 $(12 / 6 / 2010,22.19138 \mathrm{E}, 41.15289 \mathrm{~N}, 1630 \mathrm{~m}$, beech stand at treeline), Voliotis 1983: 170 (as Listera ovata (L.) R. Br.) - Sporadic. In various habitats, mainly in Pteridium thickets, up to the treeline.

?Ophrys cretica (Soó) E. Nelson X sphegodes Mill. subsp. litigiosa (E. G. Camus) Bech. - G bulb - Voliotis 1983: 170.

Ophrys scolopax subsp. cornuta (Steven) E. G. Camus - G bulb, ME - Chas. 1190 (20/5/2011, 22.22559 E, 41.11262 N, 700 m, phrygana - Juniperus oxycedrus), Chas. $1236(25 / 5 / 2011,22.21749$ E, 41.11285 N, $760 \mathrm{~m}$, opening with phrygana in thermophilous deciduous stand), Voliotis 1983: 170 - Sporadic. Grasslands, 
phrygana, openings in thermophilous deciduous stands and forest roads, up to 1100 m.

Ophrys sphegodes subsp. mammosa (Desf.) Soó ex E. Nelson - Gbulb, ME - Chas. 457 (17/4/2010, 22.22395 E, 41.11187 N, 680 m, phrygana), Voliotis 1983: 170 Common. Phrygana, dry grasslands and forest roads of lowland areas.

Orchis mascula (L.) L. - G bulb, EA - Chas. 478 (25/4/2010, 22.19838 E, 41.10616 N, $710 \mathrm{~m}$, grassy opening in thermophilous deciduous stand), $587(12 / 5 / 2010$, 22.24416 E, $41.14434 \mathrm{~N}, 1650 \mathrm{~m}$, shrubland of Juniperus communis subsp. nana at treeline), Chas. 1104 (26/4/2011, $22.19995 \mathrm{E}, 41.10961 \mathrm{~N}, 820 \mathrm{~m}$, open thermophilous deciduous shrubland), Chas. 1328 (2/6/2011, 22.24298 E, 41.14405 $\mathrm{N}, 1660 \mathrm{~m}$, grassland at treeline), Voliotis 1983: 170 (as O. mascula subsp. signifera (Vest) Soó) - Sporadic. In various habitats up to $1700 \mathrm{~m}$.

Orchis pallens L. - G bulb, ME - Chas. 559 (8/5/2010, 22.244 E, 41.15938 N, 1820 m), Voliotis 1983: 170, MFG2: 882 , - Sporadic. In subalpine grasslands.

*Orchis purpurea Huds. - G bulb, EA - Chas. 1171 (11/5/2011, 22.21402 E, 41.12217 $\mathrm{N}, 1080 \mathrm{~m}$, forest road in mixed beech and thermophilous deciduous stand) - Rare.

*Orchis simia Lam. - G bulb, EA - Chas. 1121 (1/5/2011, 22.22923 E, 41.11907 N, $1070 \mathrm{~m}$, opening in thermophilous deciduous shrubland with Juniperus oxycedrus) - Rare.

Platanthera bifolia (L.) Rich. - G bulb, Pt - Chas. 1226 (25/5/2011, 22.20775 E, $41.11171 \mathrm{~N}, 820 \mathrm{~m}$, grassy opening by roadside in thermophilous deciduous stand), Chas. 1296 (2/6/2011, 22.20162 E, 41.1226 N, 1180 m, Juniperus oxycedrus stand within beech stand), Voliotis 1983: 170 - Sporadic. Pteridium thickets and forest openings, on mesic to damp ground. Up to $1400 \mathrm{~m}$.

Platanthera chlorantha (Custer) Rchb. - G bulb, ES - Chas. 1149 (6/5/2011, 22.27748 E, 41.14583 N, $980 \mathrm{~m}$, Quercus frainetto stand), Chas. 1885 (24/5/2012, 22.21024 E, $41.11555 \mathrm{~N}, 910 \mathrm{~m}$, thermophilous deciduous stand), Voliotis 1983: 170 Sporadic. Oak forests, thermophilous deciduous stands and openings, on mesic ground.

*Spiranthes spiralis (L.) Chevall. - G rhiz, EA - Chas. 965 (26/9/2010, 22.26898 E, $41.12922 \mathrm{~N}, 770 \mathrm{~m}$, phrygana in opening of Quercus frainetto stand), Chas. 1720 (28/9/2011, 22.2208 E, 41.1093 N, 640 m, grassland) - Common. Dry grasslands and phrygana, up to $900 \mathrm{~m}$.

\section{POACEAE}

*Achnatherum bromoides (L.) P. Beauv. - H caesp, Me - Chas. 298 (10/9/2009, $22.26185 \mathrm{E}, 41.12866 \mathrm{~N}, 790 \mathrm{~m}$, forest road in oak forest) - Sporadic. Thermophilous deciduous forests and shrublands, oak forests and phrygana. Up to $1300 \mathrm{~m}$.

*Achnatherum calamagrostis (L.) P. Beauv. - H caesp, Eu - Chas. 1731 (4/10/2011, 22.22156 E, 41.12976 N, 1380 m, open Ostrya - Juniperus oxycedrus shrubland), Chas. $1743(19 / 10 / 2011,22.22227$ E, $41.13418 \mathrm{~N}, 1410 \mathrm{~m}$, opening in Ostrya stand) - Rare. Open shrublands and stands of Ostrya on calcareous substrates.

Aegilops biuncialis Vis. - T scap, MS - Chas. 1967 (4/6/2012, 22.25459 E, 41.10603 N, $600 \mathrm{~m}$, grassland), Voliotis 1983: 170 - Sporadic. Dry grasslands and forest roads of lowland areas. 
*Aegilops markgrafii (Greuter) Hammer - T scap, EM - Chas. 2150 (6/7/2012, 22.25925 E, 41.1279 N, 820 m, forest road in Quercus frainetto stand) - Sporadic. Dry grasslands and forest roads of lowland areas.

*Aegilops neglecta Bertol. - T scap, MS - Chas. 722 (2/6/2010, 22.2118 E, 41.11186 N, $790 \mathrm{~m}$, forest road in thermophilous deciduous stand), Chas. 1983 (4/6/2012, 22.25456 E, $41.11171 \mathrm{~N}, 650 \mathrm{~m}$, grassland) - Sporadic. In dry grasslands and forest roads of lowland areas.

*Aegilops triuncialis L. - T scap, MS - Chas. 721 (2/6/2010, 22.2118 E, 41.11186 N, $790 \mathrm{~m})$, Chas. $2171(11 / 7 / 2012,22.20681 \mathrm{E}, 41.11151 \mathrm{~N}, 830 \mathrm{~m})$, Chas. 2272 $(10 / 6 / 2013,22.20846$ E, 41.1119 N, 810 m) - Sporadic. Forest roads of lowland areas.

*Agrostis capillaris L. - H caesp, ES - Chas. 1637 (23/8/2011, 22.21203 E, 41.12979 $\mathrm{N}, 1360 \mathrm{~m}$, forest road in beech stand) - Rare.

*Agrostis castellana Boiss. \& Reut. - H caesp, Me - Chas. 927 (1/8/2010, 22.218 E, $41.16006 \mathrm{~N}, 1990 \mathrm{~m})$, Chas. 1581 (22/7/2011, $22.17672 \mathrm{E}, 41.15249 \mathrm{~N}, 1770 \mathrm{~m})$, Chas. $1585(22 / 7 / 2011,22.17792$ E, $41.15101 \mathrm{~N}, 1700 \mathrm{~m})$ - Common. At shrublands and grasslands at and above treeline.

*Agrostis stolonifera L. - H caesp/H rept, ES - Chas. 181 (13/8/2009, 22.18554 E, 41.12244 N, $960 \mathrm{~m}$, opening in beech stand), Chas. 949 (14/8/2010, $22.19339 \mathrm{E}$, 41.10572 N, $640 \mathrm{~m}$, streambank with Platanus stand), Chas. 1555 (17/7/2011, $22.19087 \mathrm{E}, 41.13216 \mathrm{~N}, 1360 \mathrm{~m}$, forest road in beech stand), Chas. 1652 (23/8/2011, 22.21947 E, $41.16269 \mathrm{~N}, 1890 \mathrm{~m}$, above treeline fen), Chas. 1664 (31/8/2011, $22.18799 \mathrm{E}, 41.14194 \mathrm{~N}, 1420 \mathrm{~m}$, forest road in beech stand), Chas. 1665 (31/8/2011, 22.18817 E, $41.13536 \mathrm{~N}, 1360 \mathrm{~m}$, forest road in beech stand) Common. Forest openings and roads in beech stands, Platanus stands, grasslands and damp places above treeline.

*Aira elegans Roem. \& Schult. - T scap, MS - Chas. 1295 (2/6/2011, 22.20162 E, $41.1226 \mathrm{~N}, 1180 \mathrm{~m}$, Juniperus oxycedrus stand within beech stand), Chas. 1935 (30/5/2012, 22.19717 E, 41.11148 N, 850 m, Juniperus oxycedrus and phrygana) Sporadic. in phrygana and shrublands of lowland areas.

*Anthoxanthum aristatum Boiss. - T scap, MA - Chas. 1490 (2/7/2011, 22.27738 E, 41.14583 N, 980 m, Quercus frainetto stand) - Sporadic. In oak and thermophilous deciduous stands, grasslands and forest roads of lowland areas.

*Anthoxanthum odoratum L. - H caesp, Co - Chas. 529 (2/5/2010, 22.20966 E, $41.11165 \mathrm{~N}, 800 \mathrm{~m}$, grassland), Chas. 565 (8/5/2010, $22.23116 \mathrm{E}, 41.16269 \mathrm{~N}$, $1800 \mathrm{~m}$, subalpine grassland) - Common. In grasslands all-over the mountain, but mainly above treeline.

*Apera spica-venti (L.) P. Beauv. subsp. spica-venti - T scap, ES - Chas. 1493 (2/7/2011, 22.27426 E, 41.13714 N, 880 m, Quercus frainetto stand) - Rare.

*Avena barbata Link subsp. barbata - T scap, Me - Chas. 726 (2/6/2010, 22.2118 E, $41.11186 \mathrm{~N}, 790 \mathrm{~m}$, forest road in thermophilous deciduous stand) - Sporadic. At roadsides up to $900 \mathrm{~m}$.

*Avenella flexuosa (L.) Drejer - H caesp, Co - Chas. 925 (1/8/2010, 22.21889 E, $41.16232 \mathrm{~N}, 1910 \mathrm{~m}$, subalpine shrubland), Chas. 1495 (2/7/2011, $22.27738 \mathrm{E}$, 41.14583 N, 980 m, Quercus frainetto stand), Chas. 2396 (25/7/2013, 22.20821 E, $41.16063 \mathrm{~N}, 2170 \mathrm{~m}$, subalpine grassland) - Common in grasslands and open shrublands above treeline, on siliceous substrates. Sporadic in oak and beech forests. $900-2180 \mathrm{~m}$. 
*Avenula pubescens (Huds.) Dumort. - H caesp, EA - Chas. 684 (24/5/2010, 22.25209 E, 41.15977 N, 1750 m), Chas. 730 (2/6/2010, 22.19593 E, 41.13909 N, 1770 m) Sporadic. In grasslands at and above treeline, up to $1900 \mathrm{~m}$.

Bellardiochloa variegata (Lam.) Kerguélen - H caesp, Eu - Chas. 1539 (12/7/2011, 22.22881 E, 41.15324 N, $1850 \mathrm{~m})$, Chas. 1605 (13/8/2011, 22.21231 E, 41.15871 N, 2130 m), Chas. 2077 (25/6/2012, 22.20506 E, 41.14941 N, 2010 m), MFG2: 774 - Common at grasslands above treeline. Occasionally descending to $1500 \mathrm{~m}$ in grasslands of mid-altitudes.

*Bothriochloa ischaemum (L.) Keng - H caesp, ST - Chas. 291 (10/9/2009, 22.26185 E, $41.12866 \mathrm{~N}, 790 \mathrm{~m}$, forest road in Quercus frainetto stand) - Common. In dry grasslands and phrygana of lowland areas.

*Brachypodium distachyon (L.) P. Beauv. - T scap, MS - Chas. 1429 (16/6/2011, 22.26185 E, $41.12866 \mathrm{~N}, 860 \mathrm{~m}$, phrygana) - Sporadic. At stony open places, up to $1100 \mathrm{~m}$.

*Brachypodium pinnatum (L.) P. Beauv. - H caesp, ES - Chas. 1453 (22/6/2011, 22.2081 E, $41.11208 \mathrm{~N}, 820 \mathrm{~m}$, thermophilous deciduous stand) - Common in thermophilous deciduous stands, mainly on calcareous substrates. Sporadic in grasslands up to $1700 \mathrm{~m}$.

*Brachypodium sylvaticum (Huds.) P. Beauv. subsp. sylvaticum - H caesp, Pt - Chas. $182(13 / 8 / 2009,22.18554$ E, $41.12244 \mathrm{~N}, 950 \mathrm{~m}$, beech stand), Chas. 410 $(17 / 10 / 2009,22.24754 \mathrm{E}, 41.13367 \mathrm{~N}, 1140 \mathrm{~m}$, forest road in mixed oak - beech stand), Chas. 1492 (2/7/2011, 22.27426 E, 41.13714 N, 880 m, Quercus frainetto stand) - Common in thermophilous deciduous stands and oak forests. Sporadic in beech forests at the lower part of the beech forest zone.

*Briza maxima L. - T scap, ST - Chas. 1927 (30/5/2012, 22.19801 E, 41.10651 N, 730 $\mathrm{m}$, grassy opening in thermophilous deciduous and Juniperus oxycedrus stand) Rare.

* Briza media subsp. elatior (Sm.) Rohlena - H caesp, ES - Chas. 353 (15/9/2009, 22.26424 E, $41.16013 \mathrm{~N}, 1540 \mathrm{~m}$, grassland at treeline), Chas. 1452 (22/6/2011, 22.21024 E, 41.11555 N, $910 \mathrm{~m}$, thermophilous deciduous stand) - Sporadic. Forests all-over the mountain and grasslands up to the treeline.

*Bromus benekenii (Lange) Trimen - H caesp, Pt - Chas. 2251 (1/6/2013, 22.27783 E, 41.14629 N, 980 m, grassy opening in mixed oak - beech stand) - Rare.

*Bromus cappadocicus Boiss. \& Balansa subsp. cappadocicus - H caesp, MS - Chas. $2070(21 / 6 / 2012,22.21718 \mathrm{E}, 41.14608 \mathrm{~N}, 1780 \mathrm{~m}$, rocky roadside above treeline) - Rare.

Bromus cappadocicus subsp. lacmonicus (Hausskn.) P.M. Sm. - H caesp, Bk- Chas. 277 (28/8/2009, 22.21967 E, 41.1599 N, 1930 m, subalpine grassland), Chas. 2035 (18/6/2012, 22.23207 E, 41.11542 N, 810 m, phrygana with Juniperus oxycedrus), Chas. $2285(14 / 6 / 2013,22.26979 \mathrm{E}, 41.1622 \mathrm{~N}, 1520 \mathrm{~m}$, grassland at treeline), MFG2: 789 - Common. Stony places in grasslands at mid-altitudes and above treeline. Occasionally descending to $800 \mathrm{~m}$.

Bromus hordeaceus L. subsp. hordeaceus - T scap, Co - Chas. 723 (2/6/2010, 22.2118 E, $41.11186 \mathrm{~N}, 790 \mathrm{~m}$, forest road in thermophilous deciduous stand), Chas. 1288 $(28 / 5 / 2011,22.21251 \mathrm{E}, 41.11196 \mathrm{~N}, 780 \mathrm{~m}$, forest road in thermophilous deciduous stand), Chas. 1392 (9/6/2011, 22.20072 E, 41.15123 N, $1850 \mathrm{~m}$, subalpine grassland), Chas. 1399 (9/6/2011, $22.18682 \mathrm{E}, 41.1539 \mathrm{~N}, 1600 \mathrm{~m}$, streambank with grassy vegetation at treeline), Voliotis 1983: 170 - Common in 
forest roads and dry grasslands of lowland areas. Occasionally ascending to $1900 \mathrm{~m}$ in grasslands.

*Bromus inermis Leyss. - G rhiz, ES - Chas. 1921 (30/5/2012, 22.19831 E, 41.10859 N, 780 m, streambank with Platanus in thermophilous deciduous stand) - Rare.

*Bromus ramosus Huds. - H caesp, Eu - Chas. 1663 (31/8/2011, 22.18815 E, 41.14014 $\mathrm{N}, 1400 \mathrm{~m}$, forest road in beech stand), Chas. 1708 (18/9/2011, $22.17137 \mathrm{E}$, $41.14383 \mathrm{~N}, 1450 \mathrm{~m}$, beech stand) - Sporadic. Beech stands and damp places by roadsides of beech stands.

Bromus riparius Rehmann - H caesp, EA - Chas. 2278 (14/6/2013, 22.25978 E, $41.15295 \mathrm{~N}, 1520 \mathrm{~m}$, grassy opening in beech stand), MFG2: 790 - Rare.

*Bromus squarrosus L. - T scap, Pt - Chas. 196 (24/8/2009, 22.21376 E, 41.12306 N, $1090 \mathrm{~m}$, forest road in mixed beech - thermophilous deciduous stand), Chas. 724 $(2 / 6 / 2010,22.2118 \mathrm{E}, 41.11186 \mathrm{~N}, 790 \mathrm{~m}$, forest road in thermophilous deciduous stand), Chas. 1362 (9/6/2011, 22.22255 E, 41.11068 N, 670 m, dry grassland), Chas. $1447(22 / 6 / 2011,22.20776 \mathrm{E}, 41.11162 \mathrm{~N}, 820 \mathrm{~m}$, forest road in thermophilous deciduous stand) - Common in roads of lowland areas. Sporadic in grasslands of lowland areas.

Bromus sterilis L. - T scap, MS - Chas. 645 (19/5/2010, 22.21658 E, 41.11554 N, 920 m, phrygana with Juniperus oxycedrus), Chas. 1234 (25/5/2011, 22.18864 E, $41.11817 \mathrm{~N}, 980 \mathrm{~m}$, forest road in mixed thermophilous deciduous - beech stand), Voliotis 1983: 171 - Sporadic. Roads, disturbed open ground and open shrublands at lowland areas.

*Bromus tectorum L. - T scap, Pt - Chas. 511 (2/5/2010, 22.19337 E, 41.11518 N, 910 $\mathrm{m}$, forest road in thermophilous deciduous stand), Chas. $1168(11 / 5 / 2011,22.19614$ E, $41.11387 \mathrm{~N}, 900 \mathrm{~m}$, forest road in thermophilous deciduous stand) - Common. At forest roads and other disturbed sites of lowland areas.

Calamagrostis arundinacea (L.) Roth - H caesp, EA - Chas. 82 (20/7/2009, 22.21272 E, $41.14376 \mathrm{~N}, 1750 \mathrm{~m}$, subalpine shrubland), Chas. 280 (28/8/2009, $22.2193 \mathrm{E}$, 41.14729 N, 1800 m, subalpine shrubland), Parent 2005: 228 - Common. Stony places in shrublands and grasslands at and above treeline.

*Calamagrostis epigejos (L.) Roth - H caesp, ES - Chas. 2234 (9/8/2012, 22.21309 E, 41.13449 N, 1450 m), Chas. 2394 (20/7/2013, 22.21309 E, 41.13458 N, 1450 m) Sporadic. Pteridium thickets in beech forests.

*Chrysopogon gryllus (L.) Trin. - H caesp, EA - Chas. 835 (26/6/2010, 22.22452 E, 41.11269 N, 700 m, phrygana with Juniperus oxycedrus) - Common. In grasslands and phrygana, up to $1300 \mathrm{~m}$.

*Cynodon dactylon (L.) Pers. - G rhiz, Co - Chas. 1470 (27/6/2011, 22.21725 E, $41.11294 \mathrm{~N}, 780 \mathrm{~m}$, forest road in shrubland of thermophilous deciduous and Juniperus oxycedrus) - Rare.

*Cynosurus cristatus L. - H caesp, EA - Chas. 1598 (13/8/2011, 22.21306 E, 41.14008 N, $1590 \mathrm{~m}$, damp place by roadside in beech stand) - Sporadic. Forest roads in beech stands, at the higher part of beech forest zone, and grasslands at and above treeline. Up to $1900 \mathrm{~m}$ on mesic to damp ground.

*Cynosurus echinatus L. - T scap, Me - Chas. 725 (2/6/2010, 22.2118 E, 41.11186 N, $800 \mathrm{~m}$, forest road in thermophilous deciduous stand) - Common. At forest roads and grasslands of lowland areas.

*Dactylis glomerata L. subsp. glomerata - H caesp, Pt - Chas. 180 (13/8/2009, 22.18554 E, 41.12244 N, 950 m, beech stand), Chas. 1913 (24/5/2012, 22.22666 E, 
$41.11281 \mathrm{~N}, 720 \mathrm{~m}$, phrygana with Juniperus oxycedrus) - Common. In various habitats up to the treeline.

*Danthonia decumbens (L.) DC. - H caesp, Eu - Chas. 1975 (4/6/2012, 22.20359 E, $41.11588 \mathrm{~N}, 980 \mathrm{~m}$, rocky opening in thermophilous deciduous stand) - Rare.

*Dasypyrum villosum (L.) P. Candargy - T scap, MS - Chas. 719 (2/6/2010, 22.2118 E, $41.11186 \mathrm{~N}, 790 \mathrm{~m}$, forest road in thermophilous deciduous stand) - Sporadic. At forest roads, rarely in dry grasslands, up to $900 \mathrm{~m}$.

Deschampsia cespitosa (L.) P. Beauv. subsp. cespitosa - H caesp, Co - Chas. 924 $(1 / 8 / 2010,22.21145 \mathrm{E}, 41.15969 \mathrm{~N}, 2140 \mathrm{~m}$, subalpine grassland), Chas. 1600 $(13 / 8 / 2011,22.21737 \mathrm{E}, 41.15257 \mathrm{~N}, 1850 \mathrm{~m}$, streambank with grassy vegetation above treeline), Schuler 2004: tab. 20 - Sporadic. Mesic to damp subalpine grasslands.

*Elymus caninus (L.) L. - H caesp, ES - Chas. 832 (26/6/2010, 22.21914 E, 41.12224 N, 1010 m, beech stand), Chas. 2252 (1/6/2013, 22.25772 E, 41.14067 N, $1170 \mathrm{~m}$, damp place in forest road of beech stand) - Rare.

*Elytrigia obtusiflora (DC.) Tzvelev subsp. obtusiflora - G rhiz, Eu - Chas. 302 $(10 / 9 / 2009,22.26008$ E, 41.12827 N, 810 m, forest road in Quercus frainetto stand) - Rare.

*Elytrigia repens (L.) Nevski subsp. repens - G rhiz, ES - Chas. 2161 (6/7/2012, 22.29032 E, 41.15179 N, 860 m, grassy opening in Quercus frainetto stand) - Rare.

*Festuca altissima All. - H caesp, EA - Chas. 102 (27/7/2009, 22.1844 E, 41.1335 N, $1190 \mathrm{~m})$, Chas. 1700 (13/9/2011, 22.24705 E, $41.15213 \mathrm{~N}, 1760 \mathrm{~m})$, Chas. 1741 $(19 / 10 / 2011,22.22075$ E, 41.13335 N, $1330 \mathrm{~m})$ - Sporadic. In beech forests, between 1100 and $1800 \mathrm{~m}$, mainly on calcareous substrates.

*Festuca arundinacea Schreb. subsp. arundinacea - H caesp, Pt - Chas. 2083 $(25 / 6 / 2012,22.21219 \mathrm{E}, 41.13241 \mathrm{~N}, 1350 \mathrm{~m}$, ditch by roadside in beech stand) Rare.

*Festuca arundinacea subsp. fenas (Lag.) Arcang. - H caesp, Me - Chas. 1383 (9/6/2011, 22.20728 E, 41.11143 N, 820 m, Pteridium thicket) - Sporadic. In damp open places at lowland areas.

Festuca cyllenica Boiss. \& Heldr. - H caesp, BA - Chas. 686 (24/5/2010, 22.25003 E, $41.16109 \mathrm{~N}, 1860 \mathrm{~m}$, grassland), Chas. 731 (2/6/2010, $22.20122 \mathrm{E}, 41.13737 \mathrm{~N}$, $1790 \mathrm{~m}$, grassland), Chas. 2078 (25/6/2012, $22.20271 \mathrm{E}, 41.15243 \mathrm{~N}, 1890 \mathrm{~m}$, shrubland), MFG2: 753 (as F. varia Haenke) - Common. At subalpine grasslands and shrublands.

*Festuca drymeja Mert. \& W. D. J. Koch - G rhiz, Me - Chas. 221 (24/8/2009, 22.21625 E, 41.12769 N, 1160 m), Chas. 834 (26/6/2010, 22.21914 E, 41.12224 N, 1010 m), Chas. 1748 (16/11/2011, 22.17994 E, 41.13118 N, 1050 m) - Sporadic. In beech forests. $1000-1800 \mathrm{~m}$.

*Festuca heterophylla Lam. - H caesp, Eu - Chas. 51 (11/7/2009, 22.18 E, 41.12893 N, 1000 m, beech stand), Chas. $103(27 / 7 / 2009,22.1844$ E, 41.1335 N, 1190 m, beech stand), Chas. 1506 (2/7/2011, 22.27636 E, 41.14955 N, 1030 m, Quercus frainetto stand) - Common in beech forests. Sporadic in oak forests.

*Festuca hirtovaginata (Acht.) Markgr.-Dann. - H caesp, Bk - Chas. 732 (2/6/2010, 22.20024 E, $41.13807 \mathrm{~N}, 1790 \mathrm{~m})$, Chas. 2405 (25/7/2013, 22.21071 E, 41.15634 $\mathrm{N}, 2090 \mathrm{~m})$ - Sporadic. In grasslands above treeline and at mid-altitudes.

Festuca koritnicensis Hayek \& J. Vetter - H caesp, Bk - Chas. 1341 (2/6/2011, 22.22014 E, 41.14262 N, 1610 m), Chas. 1400 (9/6/2011, 22.19069 E, 41.15621 N, 
1720 m), Chas. 1401 (9/6/2011, 22.19287 E, $41.15931 \mathrm{~N}, 1890 \mathrm{~m})$, Chas. 2388 (20/7/2013, 22.23296 E, 41.14435 N, 2030 m), MFG2: 760 - Common in subalpine grasslands, mainly on stony ground. Sporadic in grasslands of mid-altitudes.

*Festuca nigrescens Lam. - H caesp, Eu - Chas. 1606 (13/8/2011, 22.21231 E, $41.15871 \mathrm{~N}, 2130 \mathrm{~m})$, Chas. 2100 (30/6/2012, $22.22261 \mathrm{E}, 41.14806 \mathrm{~N}, 1790 \mathrm{~m})$, Chas. 2317 (22/6/2013, 22.20316 E, 41.15785 N, 1960 m), Chas. 2340 (26/6/2013, 22.22886 E, $41.16383 \mathrm{~N}, 1860 \mathrm{~m}$ ) - Common. In subalpine grasslands on mesic, siliceous ground.

Festuca paniculata L. - H caesp, Me - Chas. 785 (12/6/2010, 22.17808 E, 41.15124 N, $1710 \mathrm{~m}$ ), MFG2: 750 - Sporadic. Grasslands at and above treeline. $1700-2000 \mathrm{~m}$.

*Festuca pratensis Huds. - H caesp, EA - Chas. 2048 (21/6/2012, 22.21752 E, 41.1424 $\mathrm{N}, 1610 \mathrm{~m}$, streambank at treeline) - Rare.

Festuca rubra L. subsp. rubra - H caesp, Ct - Chas. 1574 (22/7/2011, 22.17604 E, 41.15589 N, 1830 m, streambank above treeline), Chasapis et al. 2019 - Sporadic. At damp places above treeline.

Festuca thracica (Acht.) Markgr.-Dann. - H caesp, Bk - Chas. 1260 (25/5/2011, 22.2208 E, 41.10921 N, 640 m, grassland), Chas. 1293 (28/5/2011, 22.20763 E, $41.1118 \mathrm{~N}, 820 \mathrm{~m}$, thermophilous deciduous - Juniperus oxycedrus stand), Chas. $1294(28 / 5 / 2011,22.22046 \mathrm{E}, 41.10875 \mathrm{~N}, 640 \mathrm{~m}$, grassland), Chas. 1868 (19/5/2012, 22.215 E, 41.11218 N, 770 m, phrygana with Juniperus oxycedrus), Chas. 1916 (24/5/2012, 22.22415 E, 41.11279 N, 710 m, phrygana with Juniperus oxycedrus), Chasapis et al. 2019 - Common. Thermophilous deciduous forests and shrublands and Juniperus oxycedrus shrublands.

*Festuca valesiaca Gaudin - H caesp, EA - Chas. 1223 (20/5/2011, 22.22834 E, $41.12122 \mathrm{~N}, 1170 \mathrm{~m}$, grassy opening in thermophilous deciduous stand), Chas. $1455(22 / 6 / 2011,22.20991 \mathrm{E}, 41.11544 \mathrm{~N}, 920 \mathrm{~m}$, thermophilous deciduous stand), Chas. 1456 (22/6/2011, 22.21024 E, $41.11555 \mathrm{~N}, 920 \mathrm{~m}$, thermophilous deciduous stand), Chas. 1547 (12/7/2011, 22.23082 E, $41.1536 \mathrm{~N}, 1860 \mathrm{~m}$, subalpine grassland) - Common. Thermophilous deciduous and oak stands and grasslands up to $1900 \mathrm{~m}$.

*Helictochloa aetolica (Rech. f.) Romero Zarco - H caesp, Bk - Chas. 2292 $(14 / 6 / 2013,22.26275 \mathrm{E}, 41.16245 \mathrm{~N}, 1700 \mathrm{~m}$, sparse subalpine grassland on stony ground), Chas. 2307 (18/6/2013, 22.23794 E, $41.13641 \mathrm{~N}, 1490 \mathrm{~m}$, grassland), Chas. 2344 (1/7/2013, 22.22704 E, 41.12975 N, 1570 m, grassland, Chas. 2371 (15/7/2013, 22.24509 E, $41.16291 \mathrm{~N}, 1900 \mathrm{~m}$, subalpine grassland) - Common. Stony places on calcareous substrates with grasslands at mid-altitudes and above treeline. $1300-2000 \mathrm{~m}$.

*Helictotrichon convolutum (C. Presl) Henrard - H caesp, EM - Chas. 1299 (2/6/2011, 22.19481 E, 41.11457 N, 920 m, stony forest roadside) - Rare.

*Holcus lanatus L. - H caesp, ES - Chas. 111 (27/7/2009, 22.19334 E, 41.11608 N, $950 \mathrm{~m}$, ditch by roadside in mixed beech - thermophilous deciduous), Chas. 234 (24/8/2009, 22.18985 E, 41.11746 N, 960 m) - Sporadic. Damp places (ditches by roadsides, streambanks) of lowland areas.

Hordelymus europaeus (L.) Harz - H caesp, EA - Chas. 116 (6/8/2009, 22.18389 E, 41.15205 N, $1600 \mathrm{~m})$, Chas. 150 (6/8/2009, $22.19142 \mathrm{E}, 41.14685 \mathrm{~N}, 1560 \mathrm{~m})$, Schuler 2004: map 63, Parent 2005: 233 - Common. in mesic beech stands on siliceous substrates. $1300-1750 \mathrm{~m}$. 
*Hordeum bulbosum L. - H caesp, ST - Chas. 2021 (13/6/2012, 22.22851 E, 41.12365 $\mathrm{N}, 1280 \mathrm{~m}$, grassy opening in thermophilous deciduous stand) - Rare.

*Hordeum murinum subsp. leporinum (Link) Arcang. - T scap, Me - Chas. 1837 $(11 / 5 / 2012,22.20407 \mathrm{E}, 41.11129 \mathrm{~N}, 840 \mathrm{~m}$, forest road in thermophilous deciduous stand) - Sporadic. At roadsides, up to $900 \mathrm{~m}$.

*Kengia serotina (L.) Packer subsp. serotina - H caesp/G rhiz, EA - Chas. 1732 (4/10/2011, 22.22154 E, 41.11724 N, 840 m, phrygana with Juniperus oxycedrus) Rare. Open juniper shrublands, up to $1100 \mathrm{~m}$.

Koeleria lobata (M. Bieb.) Roem. \&Schult. - H caesp, Me - Chas. 2293 (14/6/2013, 22.26275 E, 41.16245 N, $1700 \mathrm{~m})$, Chas. 2370 (15/7/2013, 22.24621 E, 41.16148 N, 1940 m), MFG2: 804 - Common. Subalpine grasslands on stony ground. On calcareous substrates between 1700 and $2050 \mathrm{~m}$.

*Koeleria macrantha (Ledeb.) Schult. - H caesp, Bo - Chas. 802 (12/6/2010, 22.21467 E, $41.11119 \mathrm{~N}, 730 \mathrm{~m}$, open shrubland of Juniperus oxycedrus and thermophilous deciduous), Chas. 1291 (28/5/2011, 22.20717 E, 41.11107 N, 810 m, Pteridium thicket), Chas. 1424 (16/6/2011, $22.22429 \mathrm{E}, 41.11242 \mathrm{~N}, 690 \mathrm{~m}$, phrygana and Juniperus oxycedrus), Chas. 1426 (16/6/2011, 22.22585 E, 41.11649 N, 880 m, open shrubland of thermophilous deciduous and Juniperus oxycedrus), Chas. 1930 (30/5/2012, 22.19551 E, 41.10665 N, 700 m, phrygana with Juniperus oxycedrus), Chas. 1968 (4/6/2012, 22.25445 E, 41.10684 N, 600 m, dry grassland) - Common in grasslands, open shrublands and phrygana of lowland areas. Occasionally descending to $2050 \mathrm{~m}$ on stony ground with sparse grasslands.

*Koeleria pyramidata (Lam.) P. Beauv. - H caesp, Eu - Chas. 1929 (30/5/2012, $22.19565 \mathrm{E}, 41.1099 \mathrm{~N}, 800 \mathrm{~m}$, open shrubland of thermophilous deciduous and Juniperus oxycedrus), Chas. 2002 (8/6/2012, 22.21794 E, 41.11826 N, 1060 m, phrygana with Juniperus oxycedrus) - Rare. Grasslands, phrygana and open shrublands of lowland areas.

*Lolium perenne L. - H caesp, ES - Chas. 1384 (9/6/2011, 22.20811 E, 41.11163 N, $810 \mathrm{~m}$, forest road in thermophilous deciduous stand) - Rare.

*Lolium rigidum Gaudin subsp. rigidum - T scap, ST - Chas. 47 (11/7/2009, 22.21882 E, $41.11215 \mathrm{~N}, 710 \mathrm{~m}$, forest road in thermophilous deciduous and Juniperus oxycedrus shrubland) - Rare.

*Melica ciliata L. subsp. ciliata - H caesp, Me - Chas. 649 (19/5/2010, 22.21465 E, $41.11641 \mathrm{~N}, 1010 \mathrm{~m}$, open grassland on stony slope), Chas. $1928(30 / 5 / 2012$, 22.19747 E, 41.10867 N, 780 m, phrygana) - Sporadic. Open shrublands, phrygana and grasslands, up to $1400 \mathrm{~m}$.

Melica uniflora Retz. - G rhiz, Pt - Chas. 1347 (9/6/2011, 22.19135 E, 41.14964 N, $1560 \mathrm{~m}$, beech stand), Schuler 2004: map 48 - Common in beech stands. Sporadic in thermophilous deciduous and oak stands. Mainly on mesic ground.

*Micropyrum tenellum (L.) Link - T scap, Me - Chas. 1894 (24/5/2012, 22.20075 E, 41.11134 N, 860 m, phrygana with Juniperus oxycedrus on stony ground) - Rare.

*Milium effusum L. - H caesp, ES - Chas. 1557 (17/7/2011, 22.19015 E, 41.13368 N, $1390 \mathrm{~m}$, beech stand), Chas. 1580 (22/7/2011, $22.17723 \mathrm{E}, 41.15377 \mathrm{~N}, 1760 \mathrm{~m}$, beech stand), Chas. 1650 (23/8/2011, $22.22101 \mathrm{E}, 41.15884 \mathrm{~N}, 1860 \mathrm{~m}$, tall-herb vegetation above treeline) - Sporadic. Beech stands and tall-herb vegetation above treeline. On mesic ground up to $2000 \mathrm{~m}$.

*Molinia arundinacea Schrank - H caesp, EA - Chas. 955 (14/8/2010, 22.22487 E, 41.11288 N, 700 m, streambank with thermophilous deciduous vegetation) - Rare. 
Molinia caerulea (L.) Moench - H caesp, ES - Chas. 1660 (31/8/2011, 22.21061 E, $41.11175 \mathrm{~N}, 800 \mathrm{~m}$, ditch by roadside in thermophilous deciduous stand), Schuler 2004: tab. 20 - Rare.

Nardus stricta L. - H caesp, ES - Chas. 278 (28/8/2009, 22.22171 E, 41.15939 N, 1850 m), Chas. 2076 (25/6/2012, 22.21004 E, 41.15012 N, 2010 m), MFG2: 832 Common. In subalpine grasslands on siliceous substrates.

Phleum alpinum L. - H caesp, AA - Chas. 783 (12/6/2010, 22.18097 E, 41.15858 N, $1820 \mathrm{~m}$, grassy opening in subalpine shrubland), Chas. 1398 (9/6/2011, 22.19228 E, $41.15894 \mathrm{~N}, 1870 \mathrm{~m}$, subalpine grassland), MFG2: 820 - Common. In subalpine grasslands.

*Phleum exaratum Griseb. subsp. exaratum - T scap, EM - Chas. 1421 (16/6/2011, 22.22266 E, 41.11086 N, 670 m, dry grassland), Chas. 1446 (22/6/2011, 22.22068 E, $41.10921 \mathrm{~N}, 640 \mathrm{~m}$, dry grassland), Chas. $1480(27 / 6 / 2011,22.21601 \mathrm{E}$, $41.11886 \mathrm{~N}, 1100 \mathrm{~m}$, open shrubland of Juniperus oxycedrus and thermophilous deciduous) - Sporadic. Grasslands, phrygana and open shrublands of lowland areas.

*Phleum hirsutum Honck. - H caesp, Eu - Chas. 569 (8/5/2010, 22.23116 E, 41.16269 N, 1800 m), Chas. 784 (12/6/2010, 22.18097 E, 41.15858 N, 1810 m), Chas. 2341 (26/6/2013, 22.22886 E, 41.16383 N, 1860 m) - Sporadic. Subalpine stony grasslands.

*Phleum phleoides (L.) H. Karst. - H caesp, ES - Chas. 1497 (2/7/2011, 22.27738 E, 41.14583 N, 980 m, Quercus frainetto stand), Chas. 1693 (13/9/2011, 22.24122 E, $41.15177 \mathrm{~N}, 1760 \mathrm{~m}$, sparse subalpine grassland on stony ground), Chas. 2286 (14/6/2013, 22.26979 E, $41.1622 \mathrm{~N}, 1520 \mathrm{~m}$, grassland at treeline) - Common. Grasslands all-over the mountain, mainly at mid-altitudes $(1200-1600 \mathrm{~m})$.

*Phleum pratense L. subsp. pratense - H caesp, ES - Chas. 301 (10/9/2009, 22.26008 E, $41.12827 \mathrm{~N}, 810 \mathrm{~m}$, forest road in Quercus frainetto stand) - Sporadic. In grasslands and forest roads of lowland areas.

*Phragmites australis (Cav.) Steud. - G rhiz/H caesp, Co - Chas. 312 (10/9/2009, 22.25447 E, 41.13756 N, 1210 m, forest road in beech stand) - Rare. At damp sites of forest roads in beech and oak forests.

*Poa angustifolia L. - G rhiz, ES - Chas. 1835 (11/5/2012, 22.20978 E, 41.11147 N, $800 \mathrm{~m}$, grassy opening in thermophilous deciduous stand) - Rare.

*Poa annua L. - T caesp, Co - Chas. 728 (2/6/2010, 22.20978 E, 41.11147 N, 1380 m, forest road in beech stand), Chas. 1759 (24/3/2012, 22.22671 E, $41.11092 \mathrm{~N}, 660$ $\mathrm{m}$, grassland) - Sporadic. Grasslands of lowland areas and forest roads up to 1400 $\mathrm{m}$.

*Poa bulbosa L. subsp. bulbosa - H caesp, Pt - Chas. 1396 (9/6/2011, 22.18998 E, $41.15593 \mathrm{~N}, 1710 \mathrm{~m}$, subalpine grassland), Chas. 1836 (11/5/2012, 22.19418 E, $41.12023 \mathrm{~N}, 1140 \mathrm{~m}$, roadside in beech stand) - Sporadic. In forests, shrublands and grasslands, up to $1750 \mathrm{~m}$.

*Poa bulbosa subsp. pseudoconcinna (Schur) Asch. \& Graebn. - H caesp, BC - Chas. 1535 (12/7/2011, 22.2298 E, 41.1531 N, 1850 m), Chas. 2338 (26/6/2013, 22.19946 E, $41.14058 \mathrm{~N}, 1890 \mathrm{~m}$ ) - Sporadic. Subalpine grasslands on stony ground.

*Poa cenisia All. - G rhiz, Eu - Chas. 2414 (30/7/2013, 22.20074 E, 41.15898 N, 1920 $\mathrm{m}$, sparse grassland on scree above treeline) - Rare. Schistose screes above treeline. Between 1700 and $1950 \mathrm{~m}$. 
*Poa compressa L. - G rhiz, Eu - Chas. 1454 (22/6/2011, 22.20887 E, 41.11443 N, 890 $\mathrm{m}$, forest road in thermophilous deciduous stand), Chas. 2431 (19/6/2014, 22.27387 E, $41.1292 \mathrm{~N}, 710 \mathrm{~m}$, oak forest) - Rare. Forests, shrublands and openings at lowland areas.

*Poa hybrida Gaudin - H caesp, BC - Chas. 135 (6/8/2009, 22.18389 E, 41.15205 N, $1590 \mathrm{~m}$, forest road in beech stand), Chas. 1672 (31/8/2011, 22.17155 E, 41.14803 $\mathrm{N}, 1670 \mathrm{~m}$, beech stand) - Common in beech stands on NW Tzena, on siliceous substrates.

*Poa macedonica (Acht.) Stoeva \& Kožuharov - H caesp, Bk - Chas. 2409 (25/7/2013, 22.2148 E, $41.1637 \mathrm{~N}, 1990 \mathrm{~m}$, swampy place above treeline), Chas. 2413 $(30 / 7 / 2013,22.21 \mathrm{E}, 41.16471 \mathrm{~N}, 2040 \mathrm{~m}$, tall-herb vegetation on damp ground above treeline) - Sporadic. In damp places above treeline.

*Poa media Schur - H caesp, BC - Chas. 1336 (2/6/2011, 22.22875 E, 41.15005 N, 1690 m), Chas. 1393 (9/6/2011, 22.20213 E, 41.16044 N, 2040 m) - Sporadic. In subalpine grasslands on siliceous substrates.

Poa molinerii Balb. - H caesp, Eu - Chas. 685 (24/5/2010, 22.25003 E, 41.16109 N, $1860 \mathrm{~m})$, Chas. 1389 (9/6/2011, $22.19228 \mathrm{E}, 41.15894 \mathrm{~N}, 1870 \mathrm{~m})$, Chas. 2027 (18/6/2012, 22.23936 E, 41.13237 N, 1340 m), Chas. 2399 (25/7/2013, 22.20978 E, $41.15534 \mathrm{~N}, 2060 \mathrm{~m}), \mathrm{MFG}$ : 773 - Common. In grasslands on stony ground, above treeline and at mid-altitudes.

*Poa nemoralis L. - H caesp, ES - Chas. 52 (11/7/2009, 22.18 E, 41.12893 N, 1000 m, beech stand), Chas. 104 (27/7/2009, 22.1844 E, $41.1335 \mathrm{~N}, 1190 \mathrm{~m}$, beech stand), Chas. 136 (6/8/2009, $22.18389 \mathrm{E}, 41.15205 \mathrm{~N}, 1590 \mathrm{~m}$, forest road in beech stand), Chas. $156(6 / 8 / 2009,22.19142 \mathrm{E}, 41.14685 \mathrm{~N}, 1560 \mathrm{~m}$, shrubland at treeline) Common. In oak and beech forests, shrublands, grasslands and Pteridium thickets at treeline.

*Poa pratensis L. - G rhiz, Ct - Chas. 1147 (1/5/2011, 22.2093 E, 41.11164 N, 800 m, grassy clearing in thermophilous deciduous stand), Chas. $1287(28 / 5 / 2011$, 22.20709 E, 41.10999 N, 780 m, grassland), Chas. 1391 (9/6/2011, 22.1972 E, $41.15352 \mathrm{~N}, 1680 \mathrm{~m}$, above treeline streambank with tall-herb vegetation) Common. Grasslands and Pteridium thickets, on mesic ground. $600-1900 \mathrm{~m}$.

Poa thessala Boiss. \& Orph. - H caesp, BA - Chas. 1388 (9/6/2011, 22.1916 E, $41.15767 \mathrm{~N}, 1790 \mathrm{~m})$, Chas. $1395(9 / 6 / 2011,22.18975 \mathrm{E}, 41.15583 \mathrm{~N}, 1700 \mathrm{~m})$, Chas. 2115 (30/6/2012, 22.21372 E, 41.16396 N, 2010 m), Chas. 2386 (20/7/2013, 22.23296 E, 41.14435 N, 2030 m), Schuler 2004: tab. 21 - Common. Subalpine stony grasslands.

*Poa timoleontis Boiss. - H caesp, EM - Chas. 648 (19/5/2010, 22.21132 E, 41.11195 $\mathrm{N}, 800 \mathrm{~m}$, forest road in thermophilous deciduous stand), Chas. 1071 (22/4/2011, 22.22454 E, 41.11179 N, 680 m, phrygana), Chas. 1159 (6/5/2011, 22.27314 E, $41.14802 \mathrm{~N}, 1130 \mathrm{~m}$, rocky opening in mixed oak - beech stand), Chas. 1397 (9/6/2011, 22.18963 E, $41.15592 \mathrm{~N}, 1700 \mathrm{~m}$, subalpine grassland on stony ground) - Common. Open stony ground up to $1800 \mathrm{~m}$.

*Poa trivialis subsp. sylvicola (Guss.) H. Lindb. - H caesp, EA - Chas. 1908 $(24 / 5 / 2012,22.22707 \mathrm{E}, 41.11102 \mathrm{~N}, 660 \mathrm{~m}$, streambank with grassy vegetation), Chas. 1955 (4/6/2012, 22.20377 E, 41.11786 N, 980 m, grassland on damp ground within a thermophilous deciduous stand) - Sporadic. Damp grasslands up to the treeline. 
*Polypogon viridis (Gouan) Breistr. - H caesp, MS - Chas. 1612 (18/8/2011, 322.20443 E, $41.12427 \mathrm{~N}, 1370 \mathrm{~m}$, roadside in beech stand) - Rare.

*Rostraria cristata (L.) Tzvelev - T caesp, Co - Chas. 1979 (4/6/2012, 22.25459 E, 41.10603 N, 600 m, grassland) - Sporadic. Dry grasslands and phrygana of lowland areas.

*Secale strictum (C. Presl) C. Presl - H caesp, MS - Chas. 944 (8/8/2010, 22.22126 E, $41.12318 \mathrm{~N}, 1000 \mathrm{~m}$, thermophilous deciduous stand on stony ground) - Rare.

?Sesleria latifolia (Adamović) Degen - Hcaesp, Bk - Voliotis 1983: 171.

Sesleria robusta Schott\&al. - H caesp, Bk - Chas. 455 (10/4/2010, 22.23015 E, 41.11575 N, 870 m, open Juniperus oxycedrus shrubland), Chas. 530 (2/5/2010, $22.20967 \mathrm{E}, 41.11165 \mathrm{~N}, 800 \mathrm{~m}$, forest road in thermophilous deciduous stand), Chas. $564(8 / 5 / 2010,22.23116 \mathrm{E}, 41.16269 \mathrm{~N}, 1800 \mathrm{~m}$, grassland on stony ground above treeline), MFG2: 780, Schuler 2004: tab. 21 - Common all-over the mountain up to $2050 \mathrm{~m}$ on stony ground with various vegetation types.

Sesleria tenerrima (Fritsch) Hayek - H caesp, Bk - Chas. 563 (8/5/2010, 22.23952 E, $41.16192 \mathrm{~N}, 1820 \mathrm{~m})$, Chas. $601(12 / 5 / 2010,22.24691 \mathrm{E}, 41.14402 \mathrm{~N}, 1660 \mathrm{~m})$, Chas. 1345 (9/6/2011, 22.20318 E, 41.16154 N, 2100 m), MFG2: 778 - Common. In sparse subalpine grasslands of stony ground.

*Stipa capillata L. - H caesp, EA - Chas. 2026 (13/6/2012, 22.22861 E, 41.11998 N, $1110 \mathrm{~m}$, grassland in thermophilous deciduous - Juniperus oxycedrus stand) Sporadic. In grasslands of mid- and low altitudes and grassy openings in thermophilous deciduous stands.

*Stipa pennata L. subsp. pennata - H caesp, ES - Chas. $2190(15 / 7 / 2012,22.24$ E, $41.15274 \mathrm{~N}, 1770 \mathrm{~m})$, Chas. 2284 (14/6/2013, $22.26979 \mathrm{E}, 41.1622 \mathrm{~N}, 1520 \mathrm{~m})$, Chas. 2294 (18/6/2013, 22.23837 E, 41.13803 N, 1530 m), Chas. 2304 (18/6/2013, $22.23882 \mathrm{E}, 41.13894 \mathrm{~N}, 1540 \mathrm{~m})$ - Common. In grasslands on calcareous substrates at mid-altitudes and above treeline. $1200-1950 \mathrm{~m}$.

Stipa pulcherrima subsp. epilosa (Martinovský) Tzvelev- H caesp, ES - Chas. 647 $(19 / 5 / 2010,22.21624$ E, $41.11932 \mathrm{~N}, 1110 \mathrm{~m}$, open shrubland of thermophilous deciduous - Juniperus oxycedrus), Chas. 2018 (13/6/2012, 22.22896 E, 41.12277 $\mathrm{N}, 1240 \mathrm{~m}$, sparse grassland), Chas. 2302 (18/6/2013, $22.24332 \mathrm{E}, 41.13559 \mathrm{~N}$, $1330 \mathrm{~m}$, grassy opening in thermophilous deciduous stand), Chas. 2308 (18/6/2013, 22.23794 E, $41.13641 \mathrm{~N}, 1490 \mathrm{~m}$, grassland), MFG2: 828 (as S. pennata subsp. pulcherrima (S. Koch) Freitag), Gonzalo 2013: 40 (as S. epilosa Martinovský subsp. epilosa) - Common. At grasslands on stony ground between 600 and 2050 m.

*Trisetum flavescens subsp. splendens (C. Presl) Arcang. - H caesp, Me - Chas. 2129 $(30 / 6 / 2012,22.22351 \mathrm{E}, 41.15024 \mathrm{~N}, 1740 \mathrm{~m}$, tall-herb vegetation at streambank above treeline), Chas. 2224 (31/7/2012, 22.17717 E, $41.15816 \mathrm{~N}, 1860 \mathrm{~m}$, tall-herb vegetation on mesic ground above treeline) - Sporadic. Tall-herb vegetation of mesic ground above treeline.

*Vulpia bromoides (L.) Gray - T caesp, Pt - Chas. 836 (26/6/2010, 22.22203 E, 41.1167 N, 830 m, phrygana) - Sporadic. Forest roads, dry lowland grasslands and phrygana.

*Vulpia ciliata Dumort. - T caesp, MS - Chas. 1286 (28/5/2011, 22.20708 E, 41.11035 N, $790 \mathrm{~m}$, Pteridium thicket at the zone of thermophilous deciduous) - Common. Dry lowland grasslands. 
*Vulpia muralis (Kunth) Nees - T caesp, - Chas. 1289 (28/5/2011, 22.2031 E, 41.10776 N, 740 m, dry grassland) - Rare.

*Vulpia myuros (L.) C. C. Gmel. - T caesp, - Chas. 1966 (4/6/2012, 22.25445 E, 41.10684 N, 600 m, dry grassland), Chas. 2447 (6/7/2014, 22.18803 E, 41.13608 $\mathrm{N}, 1360 \mathrm{~m}$, forest road in beech stand) - Sporadic. Grasslands and forest roads, up to $1500 \mathrm{~m}$.

\section{RUSCACEAE}

*Ruscus aculeatus L. - G rhiz/C frut, ME - Chas. 372 (27/9/2009, 22.20122 E, $41.11098 \mathrm{~N}, 850 \mathrm{~m}$, thermophilous deciduous stand) - Common. In oak forests and thermophilous deciduous stands.

\section{TYPHACEAE}

*Typha latifolia L. - G rhiz, Co - Chas. 1958 (4/6/2012, 22.20426 E, 41.11733 N, 980 $\mathrm{m}$, swamp in thermophilous deciduous stand) - Rare. 